\title{
Carbon Dioxide Removal from Flue Gas Using Microporous Metal Organic Frameworks
}

Final Technical Report

Reporting Start Date: 1 April 2007

Reporting End Date: $\quad 30$ June 2010

Principal Author: $\quad$ Richard Willis

Report Issued: $\quad$ October 2010

DOE Award Number: $\quad$ DE-FC26-07NT43092

Submitting Organization:

UOP LLC, a Honeywell Company

25 E. Algonquin Road

Des Plaines, IL 60017-5016

Phone: (847) 391-3190

Fax: (847) 391-3719

E-mail: richard.willis@uop.com

Subcontractors:

Douglas LeVan, Vanderbilt University, Nashville, TN, USA

Stefano Brandani, University of Edinburgh, Edinburgh, Scotland, UK

Randall Snurr, Northwestern University, Evanston, IL, USA

Adam Matzger, University of Michigan, Ann Arbor, MI, USA 
Disclaimer: "This report was prepared as an account of work sponsored by an agency of the United States Government. Neither the United States Government nor any agency thereof, nor any of their employees, makes any warranty, express or implied, or assumes any legal liability or responsibility for the accuracy, completeness, or usefulness of any information, apparatus, product, or process disclosed, or represents that its use would not infringe privately owned rights. Reference herein to any specific commercial product, process, or service by trade name, trademark, manufacturer, or otherwise does not necessarily constitute or imply its endorsement, recommendation, or favoring by the United States Government or any agency thereof. The views and opinions of authors expressed herein do not necessarily state or reflect those of the United States Government or any agency thereof."

\section{Contents}

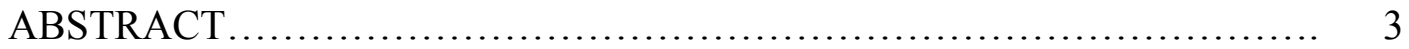

1. EXECUTIVE SUMMARY .............................................. 4

2. EXPERIMENTAL METHODS........................................... 6

3. RESULTS AND DISCUSSION ....................................... 11

3.1 MOF synthesis, scale-up and forming............................. 11

3.2 Carbon dioxide adsorption....................................... 17

3.3 Kinetics of $\mathrm{CO}_{2}$ adsorption..................................... 29

3.4 Water and steam effects on $\mathrm{CO}_{2}$ adsorption........................ 37

3.5 Fundamentals:

Effects of water, simulated flue gas, and cycling on key MOFs.. $\quad 43$

3.6 The MOF-based vPSA concept..................................... 61

3.7 Pilot Study Design .............................................. 62

3.8 Economic Analysis............................................ 62

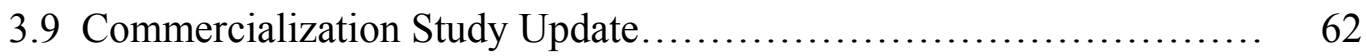

4. FINAL MILESTONE STATUS....................................... 63

5. CONCLUSIONS ...................................................... 64

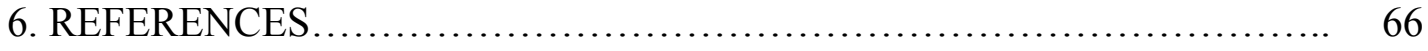

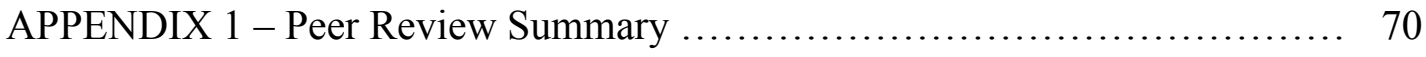

APPENDIX 2 - Process Design and Economic Analysis Report ................ 73

APPENDIX 3 - Pilot Study Design Report................................. 84

APPENDIX 4 - Updated Commercialization Study Report .................... 96 


\begin{abstract}
UOP LLC, a Honeywell Company, in collaboration with Professor Douglas LeVan at Vanderbilt University (VU), Professor Adam Matzger at the University of Michigan (UM), Professor Randall Snurr at Northwestern University (NU), and Professor Stefano Brandani at the University of Edinburgh (UE), supported by Honeywell's Specialty Materials business unit and the Electric Power Research Institute (EPRI), have completed a three-year project to develop novel microporous metal organic frameworks (MOFs) and an associated vacuum-pressure swing adsorption (vPSA) process for the removal of $\mathrm{CO}_{2}$ from coal-fired power plant flue gas. The project leveraged the team's complementary capabilities: UOP's experience in materials development and manufacturing, adsorption process design and process commercialization; LeVan and Brandani's expertise in highquality adsorption measurements; Matzger's experience in syntheis of MOFs and the organic components associated with MOFs; Snurr's expertise in molecular and other modeling; Honeywell's expertise in the manufacture of organic chemicals; and, EPRI's knowledge of power-generation technology and markets. The project was successful in that a selective $\mathrm{CO}_{2}$ adsorbent with good thermal stability and reasonable contaminant tolerance was discovered, and a low cost process for flue gas $\mathrm{CO}_{2}$ capture process ready to be evaluated further at the pilot scale was proposed.
\end{abstract}

The team made significant progress toward the current DOE post-combustion research targets, as defined in a recent FOA issued by NETL: $90 \% \mathrm{CO}_{2}$ removal with no more than a $35 \%$ increase in $\mathrm{COE}$. The team discovered that favorable $\mathrm{CO}_{2}$ adsorption at more realistic flue gas conditions is dominated by one particular MOF structure type, $\mathrm{M} / \mathrm{DOBDC}$, where $\mathrm{M}$ designates $\mathrm{Zn}, \mathrm{Co}, \mathrm{Ni}$, or $\mathrm{Mg}$ and DOBDC refers to the form of the organic linker in the resultant MOF structure, dioxybenzenedicarboxylate. The structure of the M/DOBDC MOFs consists of infinite-rod secondary building units bound by DOBDC resulting in 1D hexagonal pores about 11 angstroms in diameter. Surface areas range from 800 to $1500 \mathrm{sq} \mathrm{m} / \mathrm{g}$ for the different MOFs. $\mathrm{Mg} / \mathrm{DOBDC}$ outperformed all MOF and zeolite materials evaluated to date, with about $25 \mathrm{wt} \% \mathrm{CO}_{2}$ captured by this MOF at flue gas conditions $\left(\sim 0.13 \mathrm{~atm} \mathrm{CO}_{2}\right.$ pressure, $\left.311 \mathrm{~K}\right)$. In simulated flue gas without oxygen, the zero-length (ZLC) system was very useful in quickly simulating the effect of long term exposure to impurities on the MOFs. Detailed adsorption studies on MOF pellets have shown that water does not inhibit $\mathrm{CO}_{2}$ adsorption for MOFs as much as it does for typical zeolites. Moreover, some MOFs retain a substantial $\mathrm{CO}_{2}$ capacity even with a modest water loading at room temperature. Molecular modeling was a key activity in three areas of our earlier DOE/NETL-sponsored MOF-based research on CC. First, the team was able to effectively simulate $\mathrm{CO}_{2}$ and other gas adsorption isotherms for more than $20 \mathrm{MOFs}$, and the knowledge obtained was used to help predict new MOF structures that should be effective for $\mathrm{CO}_{2}$ adsorption at low pressure. The team also showed that molecular modeling could be utilized to predict the hydrothermal stability of a given MOF. Finally, the team showed that low moisture level exposure actually enhanced the $\mathrm{CO}_{2}$ adsorption performance of a particular MOF, HKUST-1. 


\section{EXECUTIVE SUMMARY}

UOP LLC, a Honeywell Company, in collaboration with Professor Douglas LeVan at Vanderbilt University (VU), Professor Adam Matzger at the University of Michigan (UM), Professor Randall Snurr at Northwestern University (NU), and Professor Stefano Brandani at the University of Edinburgh (UE), supported by Honeywell's Specialty Materials business unit and the Electric Power Research Institute (EPRI), have completed a three-year project to develop novel microporous metal organic frameworks (MOFs) and an associated vacuum-pressure swing adsorption (vPSA) process for the removal of $\mathrm{CO}_{2}$ from coal-fired power plant flue gas.

During Phase 1, over 35 MOF materials from the open literature and our own ideas were prepared. This satisfied a key project Milestone. In fact, we found $7 \mathrm{MOF}$ materials that met (or exceeded) the Year 1 performance targets of $15 \mathrm{wt} \% \mathrm{CO}_{2}$ capacity at $0{ }^{\circ} \mathrm{C}$ at up to 3 atmospheres pressure. These MOFs were MIL-101, MIL-53, MIL-96, Zn, Co, and $\mathrm{Ni} / \mathrm{DOBDC}$, and HKUST-1. Based on the material performance evaluation and screening conducted during Year 1, 10 MOF materials were selected for further development and testing. High carbon dioxide adsorption was determined for several of the most hydrothermally stable MOFs, satisfying another project Milestone. A preliminary commercialization study was completed in early 2009.

The objective of Phase 2 was to further develop and test up to 10 MOF materials and demonstrate one or more MOF materials with improved performance and stability that are suitable for optimization and scale-up in Phase 3. Our main focus in Phase 2 was upon the M/DOBDC series ( $\mathrm{M}=\mathrm{Mg}, \mathrm{Zn}, \mathrm{Co}$, or Ni), HKUST-1, and few others from the so-called Top Ten. At the end of Phase 2, an optimized Mg/DOBDC sample provided by UM outperformed all MOF and zeolite materials evaluated to date, as measured at VU. $\mathrm{CO}_{2}$ adsorption modeling results from NU suggest that modified versions of $\mathrm{Mg} / \mathrm{DOBDC}$ could perform even better. New systems for addressing $\mathrm{CO}_{2}$ capacity and adsorption kinetics in the presence of contaminants were constructed at UE, VU, and UM. Unfortunately, MOFs fully equilibrated with water do not pick up appreciable $\mathrm{CO}_{2}$, as first determined at UE. This means the flue gas stream would need to be dried before being passed over an adsorbent bed of Ni/DOBDC or any other MOF. Synthesis yields of many MOFs, including Ni/DOBDC and $\mathrm{Mg} / \mathrm{DOBDC}$, were improved significantly at UOP. Additional fundamental studies at UOP on HKUST-1 activation shed light on the moisture sensitivity of this MOF.

The end of Phase 2 capacity performance target was $15 \mathrm{wt} \% \mathrm{CO}_{2}$ capacity at $20{ }^{\circ} \mathrm{C}$ at up to 2 atmospheres pressure. At least six MOF materials met this performance capacity goal. The minimum stability target was retention of $75 \% \mathrm{CO}_{2}$ capacity after exposure of the material to $3-7 \mathrm{~mole} \%$ steam at $100{ }^{\circ} \mathrm{C}$ for 2 hours. Ni/DOBDC easily achieved this target, even as formed pellets.

From the 29 April 2009 Peer review, three Recommendations and one Action Item were suggested by the panel. The Recommendations were for the team (1) to focus on the stability of the adsorbents under operating conditions and adsorbent tolerance to contaminants, (2) to develop a process for more innovative water removal, and (3) to 
evaluate the effects of adding binders to MOF performance. All three of these were addressed by the team in Phase 3. The single Action Item from the Peer Review was for the team to determine a means toward achieving the DOE Cost of Electricity (COE) increase goal. The team has determined that progress toward minimizing the $\mathrm{COE}$ can be made by improving the best MOFs in order to increase $\mathrm{CO}_{2}$ capacity, and optimizing the vPSA process and overall flue gas processing conditions. A final response to the April 2009 Peer Review Action Item appears in Appendix 1.

The objective of Phase 3 was to demonstrate that one or more MOF materials could meet performance targets and have sufficient stability to carry into pilot testing. End of Phase 3 stability performance targets of retention of up to $75 \%$ of $\mathrm{CO}_{2}$ capacity after exposure to up to $15 \mathrm{~mol} \%$ steam at $150{ }^{\circ} \mathrm{C}$ for four hours on Ni/DOBDC pellets and extrudates was not achieved. Only about $45 \%$ capacity was retained. Note that in the actual application, the MOF adsorbent will never be exposed to such harsh steaming. This set of conditions, selected somewhat arbitrarily over three years ago, was obviously too extreme to be achievable with Ni/DOBDC. Nevertheless, the material was not completely destroyed by the extreme steaming conditions. This suggests that MOFs still hold great promise in this application provided they are protected from moisture.

The Project was extended by three months to allow UM time to generate $20 \mathrm{~g}$ of the team's Number $1 \mathrm{MOF}, \mathrm{Mg} / \mathrm{DOBDC}$, for forming and adsorption studies. $\mathrm{ZLC} \mathrm{CO}_{2}$ capacity measurements on a fresh $\mathrm{Mg} / \mathrm{DOBDC}$ sample confirm that this material is the best material for CC, but additional ZLC experiments in simulated flue gas suggest that $\mathrm{Mg} / \mathrm{DOBDC}$ is more moisture sensitive than Ni/DOBDC. VU confirmed these results in cyclic water and $\mathrm{CO}_{2}$ adsorption experiments. In fact, moderate (end of Year 2 type) steaming of $\mathrm{Mg} / \mathrm{DOBDC}$ decreased its $\mathrm{CO}_{2}$ capacity by 50 to $60 \%$. This is in contrast to the 25 to $27 \% \mathrm{CO}_{2}$ capacity loss on $\mathrm{Ni} / \mathrm{DOBDC}$. Interestingly, $\mathrm{Ni} / \mathrm{DOBDC}$ can be regenerated easily after exposure for short to moderate time in simulated flue gas. Finally, the team confirmed via independent approaches at UE and VU that macropore diffusion is the critical mass transfer mechanism of adsorption for HKUST-1, $\mathrm{Ni} / \mathrm{DOBDC}$ and $\mathrm{Mg} / \mathrm{DOBDC}$. 


\section{EXPERIMENTAL METHODS}

In this section, example Experimental Methods for key steps in the Project will be described. These include MOF synthesis and characterization, hydrothermal stability determination procedures, model development for hydrothermal stability, model development for adsorption isotherms of gas mixtures, a gravimetric apparatus for determining adsorption isotherms, the ZLC apparatus for determining adsorption isotherms and kinetics, a concentration-swing frequency response (CSFR) apparatus for determining adsorption kinetics, and a gas-flow system for breakthrough experiments.

MOF synthesis (UM). Some detailed MOF synthesis procedures are provided in the REFERENCES section. An example, for MOF-177 ${ }^{1}$, is provided here: $\mathrm{Zn}\left(\mathrm{NO}_{3}\right)_{2} \cdot 6 \mathrm{H}_{2} \mathrm{O}$ $(0.368 \mathrm{~g})$ and 4,4',4"-benzene-1,3,5-triyl-tri-benzoic acid $\left(\mathrm{H}_{3} \mathrm{BTB}\right)(0.180 \mathrm{~g})$ were dissolved in DEF $(10 \mathrm{~mL})$ in a $20 \mathrm{~mL}$ vial, capped tightly, and heated to $100{ }^{\circ} \mathrm{C}$ for 20 hours. The solution was decanted, and the clear block crystals were washed in DMF and exchanged with $\mathrm{CHCl}_{3}$ thrice in three days. The material was evacuated at $125{ }^{\circ} \mathrm{C}$ for 6 hours before additional analysis.

Improved $\mathrm{Mg} / \mathrm{DOBDC}$ preparation (UM). Linker precursor 2,5-dihydroxyterephthalic acid (2.98 g) was dissolved in $180 \mathrm{~mL} 1$-methyl-2-pyrrolidinone (NMP) $+20 \mathrm{~mL}$ deionized water using sonication. $\mathrm{Mg}(\mathrm{OAc})_{2} \cdot 4 \mathrm{H}_{2} \mathrm{O}(6.44 \mathrm{~g})$ was added to a $0.5 \mathrm{~L}$ jar. The 2,5-dihydroxyterephthalic acid solution was filtered into the jar. The solution was sonicated to completely dissolve the magnesium acetate and the jar was placed in a 120 ${ }^{\circ} \mathrm{C}$ oven for 20 hours. The mother liquor was decanted while still hot and the yellow solid was washed with methanol $(3 \times 200 \mathrm{~mL})$. The methanol was decanted and replaced with fresh methanol $(200 \mathrm{~mL}) 3$ times over the course of 3 days. The yellow solid was evacuated at $250{ }^{\circ} \mathrm{C}$ for 16 hours and then transferred to a glove box for storage. One batch produced $\sim 3 \mathrm{~g}$ of $\mathrm{Mg} / \mathrm{DOBDC}$.

MOF characterization (UOP). Powder X-ray diffraction (XRD) patterns were obtained on a Scintag XDS $2000(\mathrm{CuK} \alpha)$ over the range of $2-56^{\circ} 2 \theta$ with a stepsize of $0.02^{\circ} 2 \theta$ and a one second integration time per step. Elemental analysis was accomplished by ICP and Leco $(C, N)$. Sample surface area and pore volume measurements were determined from $\mathrm{N}_{2}$ 40-point isotherm data using a Micromeritics ASAP 2400 instrument. Samples were pretreated at up to $300{ }^{\circ} \mathrm{C}$ in vacuum before analysis. Microscopic characterization was done on a high-resolution JEOL 7401. FTIR measurements were performed in an automated flow system apparatus on $\sim 10 \mathrm{mg}$ pressed pellets of MOF samples.

Hydrothermal stability determination procedures (UOP). Hydrothermal stability is determined in an "accelerated" manner by exposing MOFs to steam at significantly higher concentration and at higher temperature than would be expected in the flue gas application. For example, while steam levels may only reach a few mole percent at temperatures up to perhaps $50{ }^{\circ} \mathrm{C}$ in flue gas, we evaluate materials at up to $50 \mathrm{~mole} \%$ steam and up to $350{ }^{\circ} \mathrm{C}$. A high throughput apparatus, as shown and described in Figure 1, is utilized for the steaming work. After steaming, samples are subjected to X-ray diffraction (XRD) analysis to determine structural stability. 


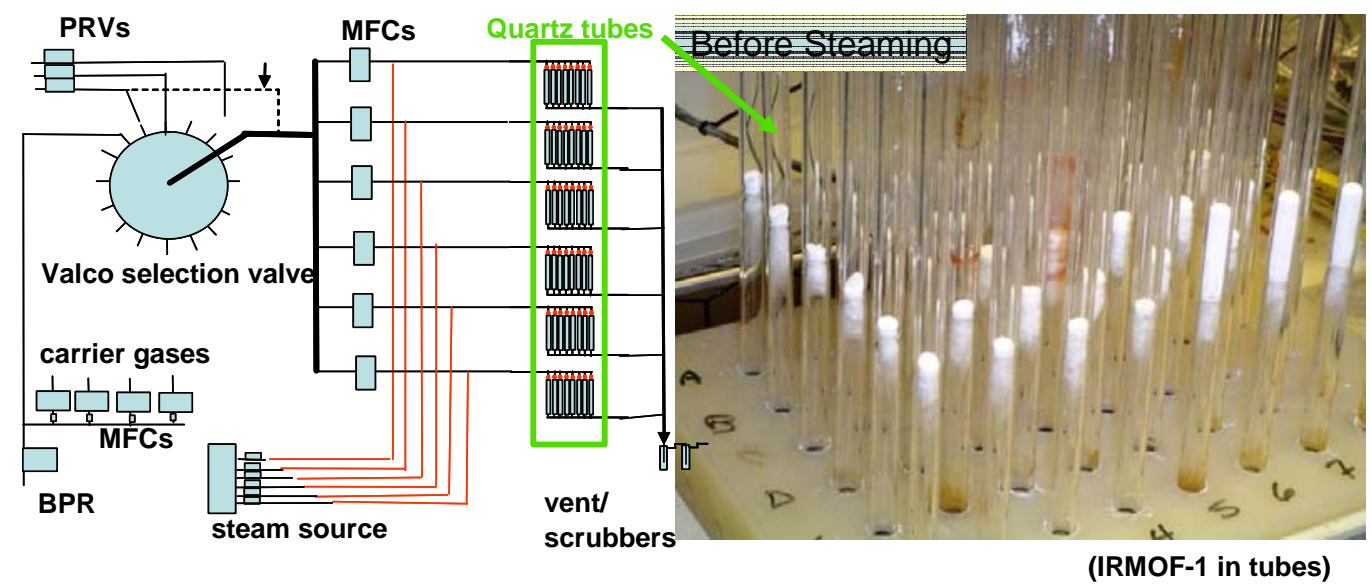

Figure 1: High throughput heat treatment unit (HTU)schematic and actual image.

Model for hydrothermal stability (UOP). Quantum mechanics were used to model the reaction of water with metal organic frameworks to avoid force fields issues. Quantum mechanics does not require parameters for every metal ligand pair and can model hydrolysis reactions which involve the breaking of O-H bonds. Unfortunately, quantum mechanics requires orders of magnitude more computational effort than force-field calculations. However, MOFs are amenable to simplified cluster studies since much of the organic linker is not directly involved in the reaction between water and metal oxide clusters. A simple model of MOF connectivity can be built by replacing a linking ligand with a much simpler linker containing the same functional group bound to the metal. For example, one can replace terephthalate with acetate in MOF-5 to yield the $\mathrm{Zn}_{4} \mathrm{O}\left(\mathrm{O}_{2} \mathrm{CCH}_{3}\right)_{6}$ cluster. Thus the $\mathrm{Zn}_{4} \mathrm{O}\left(\mathrm{O}_{2} \mathrm{CH}_{3}\right)_{6}$ cluster should have similar chemistry to the $\mathrm{Zn}_{4} \mathrm{O}\left(\mathrm{O}_{2} \mathrm{CR}\right)_{6}$ clusters in the IRMOF series. An added benefit is that MOF frameworks are flexible, which suggests that the framework may not impose significant constraints on the metal oxide cluster during reactions. This framework flexibility also implies that a cluster approach to modeling the chemistry of MOFs may be valid.

Model for isotherms of gas mixtures (NU). Grand Canonical Monte Carlo simulations were used to calculate the single-component mixture isotherms. The input fugacities were calculated using the Peng-Robinson equation of state. For the single-component simulations, the GCMC moves included random translation, rotation, reinsertion of an existing molecule, insertion of a new molecule, and deletion of an existing molecule. In addition to these moves, a Monte Carlo move that swapped the identity of existing molecules was included for the mixture simulations. A total of $10^{5}$ cycles were performed for each simulation, where a cycle includes $N$ Monte Carlo moves, with $N$ being the number of adsorbate molecules (which fluctuates). The isosteric heats of adsorption at infinite dilution $\left(Q_{s t}{ }^{0}\right)$ were calculated from NVT simulations with a single adsorbate molecule. The Monte Carlo trial moves in these simulations included random translation, rotation, and molecule reinsertion. At infinite dilution, $Q_{s t}{ }^{0}$ can be calculated as 
$Q_{s t}{ }^{0}=R T-<V>$

where $<\mathrm{V}>$ is the average potential energy per adsorbate molecule and $\mathrm{R}$ is the gas constant.

Gravimetric apparatus for determining adsorption isotherms (VU). The team used a Rubotherm gravimetric system shown schematically in Figure 2 to measure pure $\mathrm{CO}_{2}$ isotherms for different MOFs. All samples were regenerated before isotherm measurements.

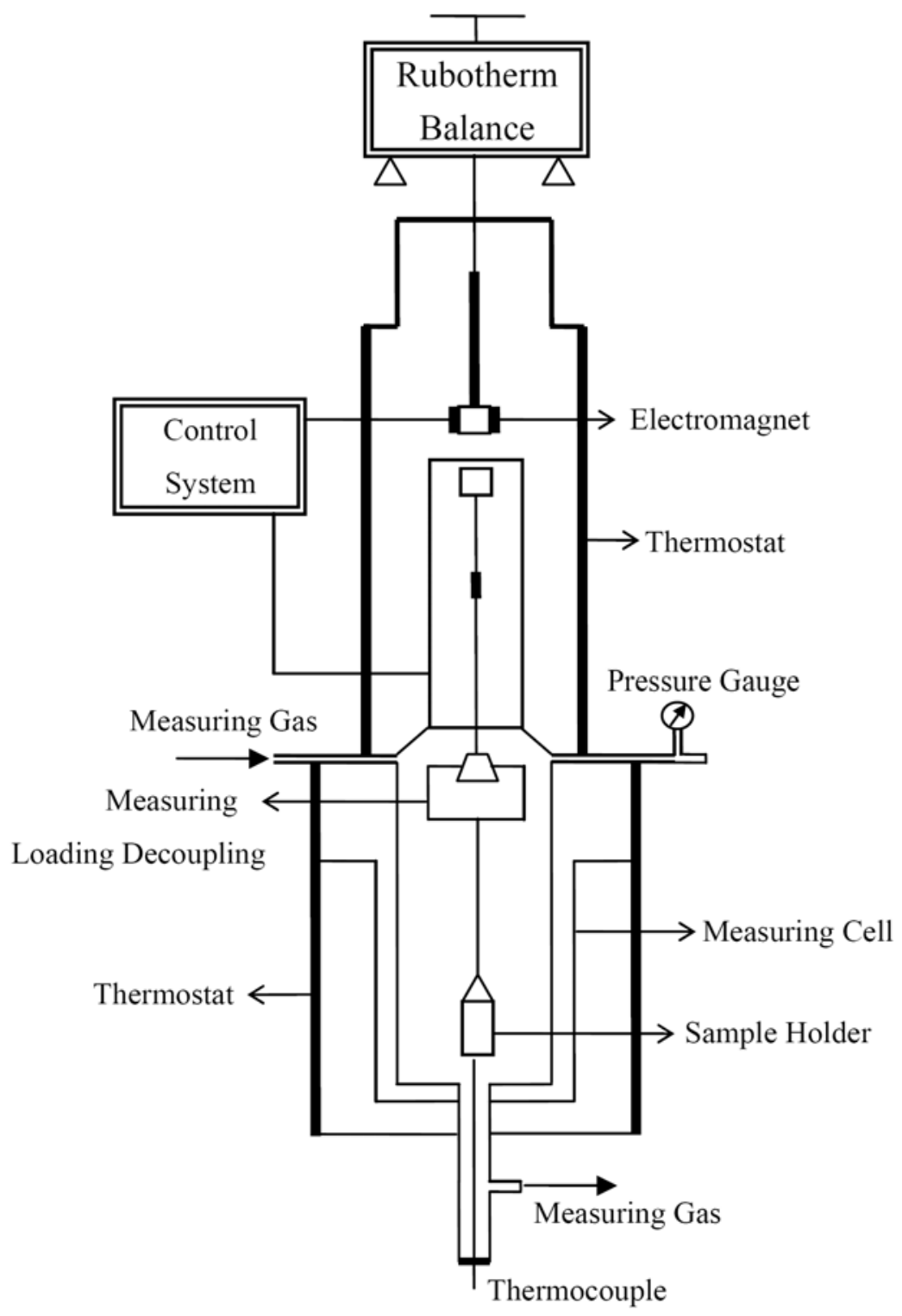

Figure 2. Schematic of Rubotherm gravimetric system for $\mathrm{CO}_{2}$ isotherm measurements. 
The ZLC apparatus for determining adsorption isotherms and kinetics (UE). The apparatus includes gas dryers, a gas dosing system, a preheat oven, a set of four on-off valves to switch the gas flows, the ZLC oven and the detector. The gas dosing system consists of four cylinders inside an oven to allow mixtures to be prepared that contain water or other vapors. Water can be dosed by pulling a vacuum in the cylinders and opening the needle valve above the capsule. Weighing the capsule before and after adding water to the system, using a Mettler-Toledo balance, it is possible to have an accurate measurement of the mass of water added and adding $\mathrm{CO}_{2}$ and $\mathrm{He}$ to the cylinders the partial pressure of water can be adjusted to the desired amount. A digital pressure transducer is connected directly above the oven in order to eliminate cold spots. Figure 3 shows the ZLC apparatus with the mass flow controllers (top left), the pre-heat oven (left); the ZLC oven (right) and the TCD and DID detector control boxes (right). Partly visible (behind the units to the right) is also the Ametek quadrupole mass spectrometer used for the stability tests. The TCD detector is mounted above the ZLC. The two ovens are controlled by two independent control units (bottom center). Needle valves are connected after the ZLC and on the vent line to balance the pressure in order to avoid uneven flows when the valves (mounted below the horizontal plate) are switched. The differential pressure transducer read-out is visible below the mass flow controllers.

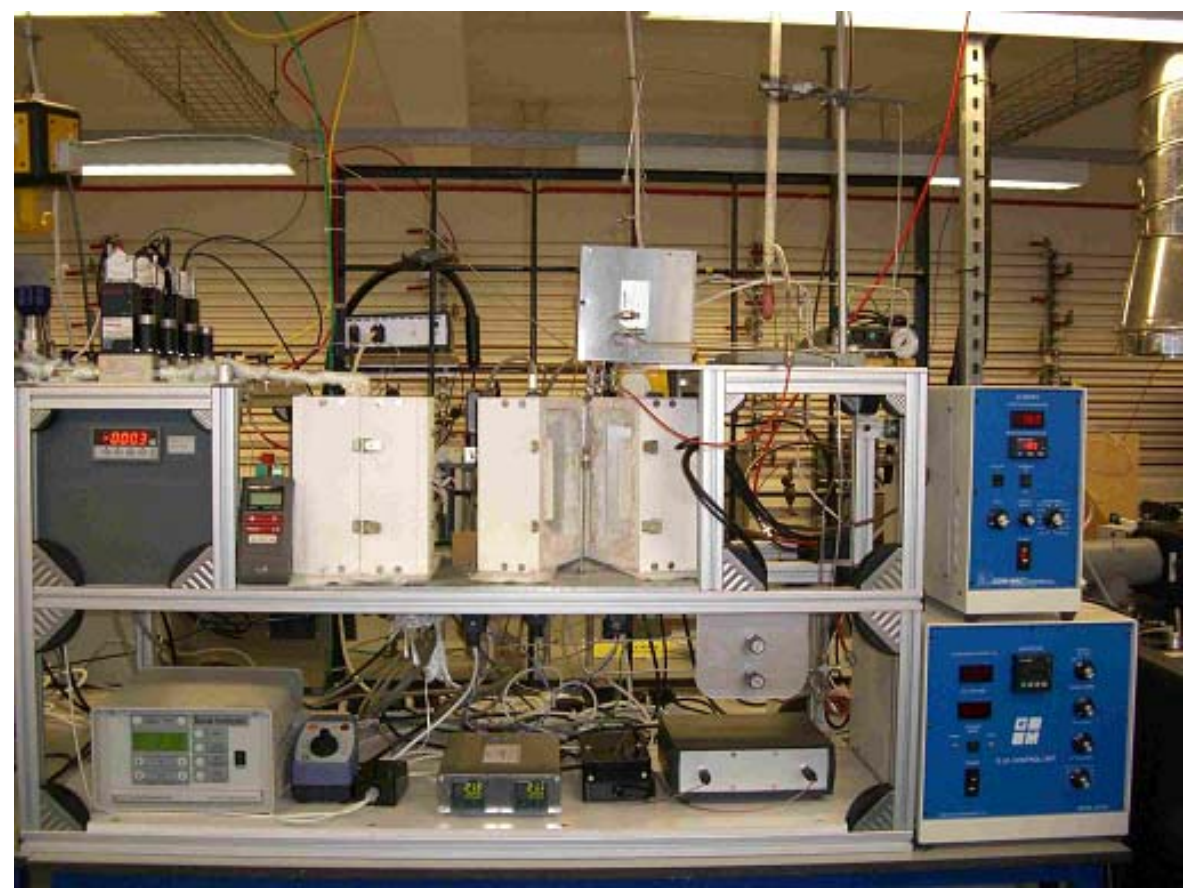

Figure 3. Front view of the ZLC apparatus.

The CSFR apparatus for determining adsorption kinetics (VU). Figure 4 shows a diagram of the concentration-swing frequency response (CSFR) system that was used to study the $\mathrm{CO}_{2}$ adsorption rates in HKUST-1 and Ni/DOBDC pellets. The $\mathrm{CO}_{2}$ gas was fed to the system at a mean flow rate of $1 \mathrm{sccm}$ with a sinusoidal perturbation of $0.5 \mathrm{sccm}$ amplitude. Simultaneously, helium was fed to the system at a mean flow rate of $49 \mathrm{sccm}$ with the sinusoidal perturbation of $0.5 \mathrm{sccm}$ amplitude but reversed in phase. The 
resulting mixtures, having a constant flow rate of $50 \mathrm{sccm}$ but a sinusoidally modulated $\mathrm{CO}_{2}$ concentration, were passed through the shallow adsorption bed packed with MOF pellets and were vented via a vacuum pump. The flow rate was controlled by a mass flow controller (MKS1479A). The system pressure was maintained by a pressure controller (MKS 640) downstream of the adsorption bed. The inlet and outlet concentrations of $\mathrm{CO}_{2}$ were analyzed by amass spectrometer (HP 5971A), and amplitude ratios of the outlet concentration to the inlet concentration were used to extract mass transfer parameters.

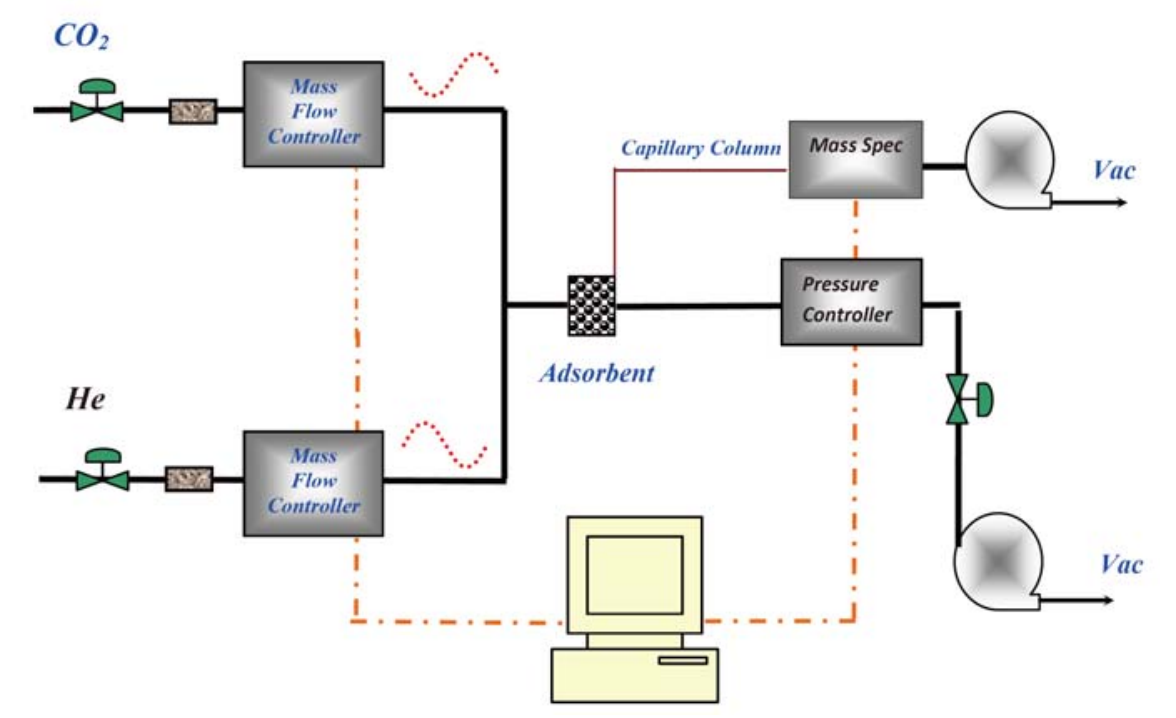

Figure 4. CSFR apparatus for $\mathrm{CO}_{2}$ adsorption rate study

Gas-flow breakthrough unit (UM). A custom-built gas flow apparatus (Figure 5) was constructed for studying the effect of humidity on the $\mathrm{CO}_{2}$ capacities of materials in the $\mathrm{M} / \mathrm{DOBDC}$ series (where $\mathrm{M}=\mathrm{Zn}, \mathrm{Co}, \mathrm{Ni}, \mathrm{Mg}$; $\mathrm{DOBDC}=2,5$-dioxido-1,4 benzenedicarboxylate).

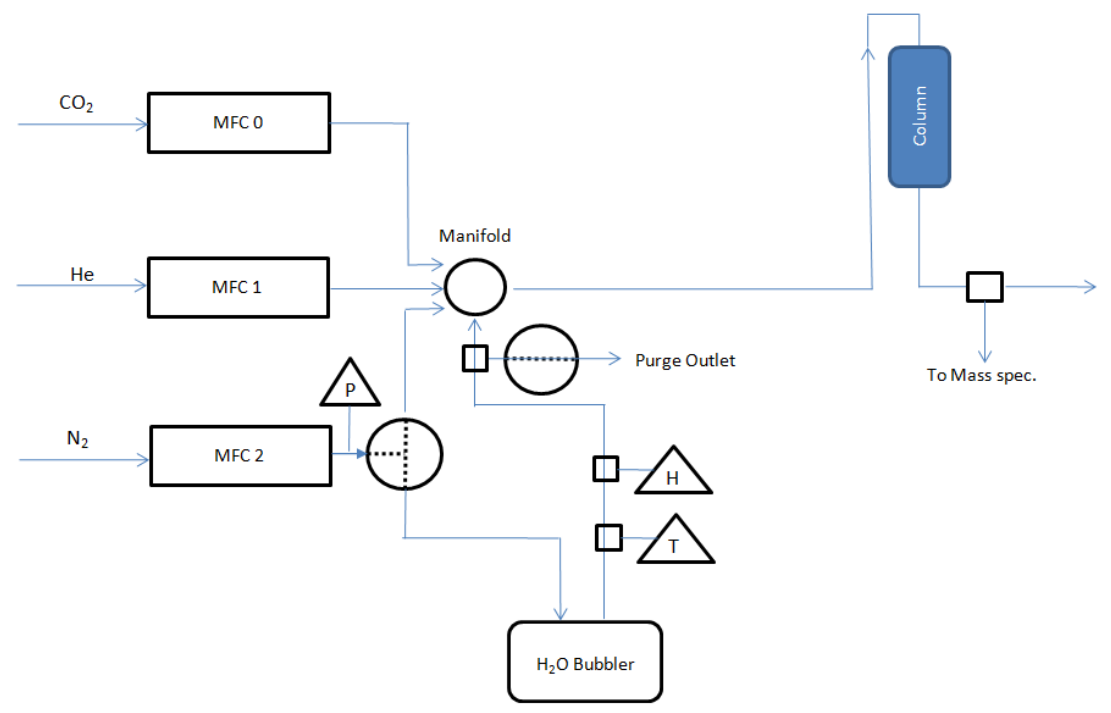

Figure 5. The gas-flow apparatus. 
The apparatus has several features that were absent in an earlier system, including:

- A radial manifold block equipped with on-off solenoid valves, four input ports, and a common output port; the on-off valves allow unused gas lines to be isolated from active lines, thereby preventing back-mixing

- A water bubbler for humidification of $\mathrm{N}_{2}$ gas

- A pressure transducer, humidity sensor, and two RTD sensors for quantifying water uptake

- A 2-way solenoid valve that can be opened to prevent pressure buildup during bubbler purges

- A 3-way solenoid valve for directing $\mathrm{N}_{2}$ either to the manifold for $\mathrm{N}_{2} / \mathrm{CO}_{2}$ breakthrough, or to the bubbler for $\mathrm{N}_{2} / \mathrm{CO}_{2} / \mathrm{H}_{2} \mathrm{O}$ breakthrough

\section{RESULTS AND DISCUSSION}

\subsection{MOF synthesis, scale-up, and forming}

The objective of this project was to develop a low cost novel sorbent (MOF) and the process around it (vPSA) to capture $\mathrm{CO}_{2}$ from coal-based power plant flue gas in a costeffective manner. The key characteristics for a cost-effective process are good sorption properties (selectivity, capacity, kinetics), low energy requirement for regeneration, good thermal and oxidative stability, tolerance to contaminants, and low cost.

The project was divided into three distinct, year-long Phases. In Phase 1, we made over 35 known MOF materials and systematically evaluated their hydrothermal stability at typical and extreme operational conditions. Those materials passing hydrothermal stability screening with high $\mathrm{CO}_{2}$ capacity at lower temperature were tested for $\mathrm{CO}_{2}$ adsorption capacity at higher, more typical operational temperatures. MOF syntheses successfully reproduced from the open literature, or samples provided from original research groups are shown in Table 1. These materials have been prepared at least twice, with the second preparation a scale-up to the at least one-gram scale. Samples prepared successfully (reasonable yield and high purity) were then provided to collaborators at VU and UE for adsorption studies. 
Table 1. MOF syntheses successfully reproduced from the open literature, or samples provided from original research groups.

\begin{tabular}{|c|c|c|c|c|}
\hline MOF & reference & metal & linker(s) & interesting property \\
\hline IRMOF-1 & 2 & $\mathrm{Zn}$ & benzenedicarboxilic acid (BDC) & very high surface area \\
\hline nano-IRMOF-1 & 3 & $\mathrm{Zn}$ & $\mathrm{BDC}$ & very high surface area, easier prep, nano \\
\hline Zn-MOF-74 & 4 & $\mathrm{Zn}$ & dihydroxy-BDC & high surface area, different structure \\
\hline MOF-508 & 5 & $\mathrm{Zn}$ & BDC plus & pillar effect, interwoven structure \\
\hline HKUST-1 & 6 & $\mathrm{Cu}$ & benzenetricarboxylic acid (BTC) & coordinatively unsaturated metal \\
\hline MIL-53 & 7 & $\mathrm{Al}$ & $\mathrm{BDC}$ & high thermal stability \\
\hline MIL-96 & 8 & $\mathrm{Al}$ & BTC & high thermal stability \\
\hline MIL-101 & 9 & $\mathrm{Cr}$ & $\mathrm{BDC}$ & very high surface area \\
\hline MAMS-1 & 10 & $\mathrm{Ni}$ & 5-t-Bu-1,3-benzenedicarboxylate & temp tunable pore size \\
\hline ZIF-8 & 11 & $\mathrm{Zn}$ & methylimidazole & zeolite-like structure \\
\hline $\mathrm{Pt} / \mathrm{Y} \mathrm{MOF}$ & 12 & $\mathrm{Y}$ & bipyridine-dicarboxylate (BPDC) & bimetallic, coordinatively unsaturated metal \\
\hline MIL-47 & 13 & $\mathrm{~V}$ & $\mathrm{BDC}$ & high $\mathrm{CO} 2$ affinity \\
\hline ZMOF-Rho & 14 & In & imidazoledicarboxylic acid & anionic framework \\
\hline Dy-btc & 15 & Dy & BTC & high $\mathrm{CO} 2$ affinity at $273 \mathrm{~K}$ \\
\hline Ln-pda & 16 & lanthanides & 1,4-phenylenediacetic acid & small pores \\
\hline Mn-formate & 17 & $\mathrm{Mn}$ & formate & small pores \\
\hline IRMOF-3 & 18 & $\mathrm{Zn}$ & amino-BDC & basic linker \\
\hline IRMOF-111 & 19 & $\mathrm{Zn}$ & bromo-BDC & bulky halide on linker \\
\hline Zn-IDC & 20 & $\mathrm{Zn}$ & imidazoledicarboxylic acid and piperizine & all five-coordinate zinc \\
\hline Pd-pymo & 21 & $\mathrm{Pd}$ & 2-hydroxypyrimidine & noble metal cornerstones \\
\hline $\mathrm{Co} / \mathrm{DOBDC}$ & 22 & $\mathrm{Co}$ & dihydroxy-BDC & high surface area, different structure \\
\hline $\mathrm{Ni} / \mathrm{DOBDC}$ & 23 & $\mathrm{Ni}$ & dihydroxy-BDC & high surface area, different structure \\
\hline Al-MIL-110 & 24 & $\mathrm{Al}$ & BTC & different $\mathrm{Al}$ coordination \\
\hline Ni-bpe & 25 & $\mathrm{Ni}$ & 1,2-bis(4-pyridyl)ethane (bpe) & selective for $\mathrm{CO} 2$ over $\mathrm{N} 2$ \\
\hline MOF-69C & 26 & $\mathrm{Zn}$ & $\mathrm{BDC}$ & 4 and 6 coordinate zinc \\
\hline MOF-144 & 26 & $\mathrm{Zn}$ & $\mathrm{BDC}$ & 4 and 6 coordinate zinc \\
\hline $\mathrm{PCN}-5$ & 27 & $\mathrm{Ni}$ & 4,4',4"-s-triazine-2,4,6-triyl-tribenzolate (TATB) & high surface area, different structure \\
\hline $\mathrm{Pt} / \mathrm{Zn}-\mathrm{MOF}$ & 28 & $\mathrm{Zn}$ & pyridine-3,5-dicarboxylic acid & bimetallic, unsaturated metal \\
\hline MIL-53calc & 7 & $\mathrm{Al}$ & $\mathrm{BDC}$ & optimized activation \\
\hline UMCM-1 & 29 & $\mathrm{Zn}$ & $\mathrm{BDC}$ and $\mathrm{BTB}$ & micro- and mesoporosity \\
\hline Tb-MOF-76 & 4 & $\mathrm{~Tb}$ & $\mathrm{BDC}$ & medium sized channels \\
\hline $\mathrm{Mg} / \mathrm{DOBDC}$ & 30 & $\mathrm{Mg}$ & dihydroxy-BDC & high surface area, different structure \\
\hline $\mathrm{PCN}-13$ & 31 & $\mathrm{Zn}$ & TATB & high $\mathrm{CO} 2$ affinity \\
\hline ZIF-95 & 32 & $\mathrm{Zn}$ & 5-chlorobenzimidazolate & high hydrothermal stability \\
\hline CUK-1 & 33 & Co & 2,4-pyridinedicarboxylate & selective for $\mathrm{CO} 2$ over $\mathrm{N} 2$ \\
\hline UMCM-150 & 34 & $\mathrm{Cu}$ & biphenyl-3,4',5-tricarboxylate (BPTC) & high surface area, different structure \\
\hline UMCM-150A & na & $\mathrm{Cu}$ & pyrimidal-phenyl TC & electron deficient UMCM-150 \\
\hline Zn-bdc-DABCO & 35 & $\mathrm{Zn}$ & $\mathrm{BDC}$ and $\mathrm{DABCO}$ & pillar effect, non-interwoven structure \\
\hline Ga-MIL-68 & 36 & $\mathrm{Ga}$ & $\mathrm{BDC}$ & Kagome effect \\
\hline Zr-UiO-66 & 37 & $\mathrm{Zr}$ & $\mathrm{BDC}$ & strong $\mathrm{Zr}$ (IV) metal oxygen bonds \\
\hline Ti-MIL-125 & 38 & $\mathrm{Ti}$ & $\mathrm{BDC}$ & strong Ti (IV) metal oxygen bonds \\
\hline
\end{tabular}


MOFs from the literature. Some examples prepared at UOP are described in this section.

M/DOBDC MOFs. M/DOBDC MOFs show very high $\mathrm{CO}_{2}$ uptake capacity at the point of interest (POI, typically for this Project the POI is at $0.1 \mathrm{~atm}$ at $311 \mathrm{~K}$ ) and good hydrothermal stability and were selected as materials of primary focus. (Surface area values reported below in parentheses are derived from BET and Langmuir methods, respectively, in $\mathrm{m}^{2} / \mathrm{g}$.)

A systematic study of the effect of synthesis method on yield and surface area was conducted to identify optimal synthesis conditions for material scale up. In the $\mathrm{THF} /$ water solvent system, which uses acetate salts, Co and Ni/DOBDC at $2 \mathrm{x}$ and $4 \mathrm{x}$ concentrations were synthesized. In the DMF/EtOH/water solvent system, which uses nitrate salts, $\mathrm{Co}, \mathrm{Ni}$, and $\mathrm{Mg} / \mathrm{DOBDC}$ materials at $2 \mathrm{x}$ and $4 \mathrm{x}$ concentrations were synthesized. Yield findings are summarized below, normalized to $\mathrm{mg} / \mathrm{mL}$ solvent used:

\begin{tabular}{|l|c|c|c|c|c|}
\hline THF/water & Conc. & $\begin{array}{c}\text { Yield } \\
(\mathrm{mg} / \mathrm{mL})\end{array}$ & $\begin{array}{l}\text { DMF/EtOH/water } \\
\text { Conc. }\end{array}$ & $\begin{array}{c}\text { Yield } \\
(\mathrm{mg} / \mathrm{mL})\end{array}$ \\
\hline $\mathrm{Co} / \mathrm{DOBDC}$ & $2 \mathrm{x}$ & 0.8 & $\mathrm{Co} / \mathrm{DOBDC}$ & $2 \mathrm{x}$ & 0.463 \\
\hline $\mathrm{Co} / \mathrm{DOBDC}$ & $4 \mathrm{x}$ & 1.73 & $\mathrm{Co} / \mathrm{DOBDC}$ & $4 \mathrm{x}$ & 1.343 \\
\hline $\mathrm{Ni} / \mathrm{DOBDC}$ & $2 \mathrm{x}$ & 2.47 & $\mathrm{Ni} / \mathrm{DOBDC}$ & $2 \mathrm{x}$ & 0.537 \\
\hline $\mathrm{Ni} / \mathrm{DOBDC}$ & $4 \mathrm{x}$ & 4.73 & $\mathrm{Ni} / \mathrm{DOBDC}$ & $4 \mathrm{x}$ & 1.612 \\
\hline & & $\mathrm{Mg} / \mathrm{DOBDC}$ & $2 \mathrm{x}$ & 0.28 \\
\cline { 4 - 7 } & & \multirow{2}{*}{$\mathrm{Mg} / \mathrm{DOBDC}$} & $4 \mathrm{x}$ & 0.82 \\
\cline { 4 - 6 } & & &
\end{tabular}

It is evident that the THF/water solvent system produces a much higher yield at both concentrations than M/DOBDC synthesized in the DMF/EtOH/water system. XRD patterns (not shown) also suggest higher quality products for the THF/water solvent system.

$\mathrm{Ni} / \mathrm{DOBDC}$. The nickel member of the DOBDC family is high yielding, has the second highest $\mathrm{CO}_{2}$ uptake capacity at the point of interest, good hydrothermal stability, and is relatively easy to activate. Pellet and powder material were steamed $\left(5 \%, 100{ }^{\circ} \mathrm{C}, 2 \mathrm{~h}\right)$ in the HTU and sent out to collaborators at VU and UE. Extrudates were also made and sent out. Mercury porosimetry results showed steamed pellet $>$ unsteamed pellet $>$ unsteamed extrudate for total pore area, intrusion volume, and porosity (Figure 6 left). This suggests that steaming has opened up mesoporosity within the pellets. Highresolution SEM images show globular aggregates of tabular prismatic crystals. (Figure 6 right). 


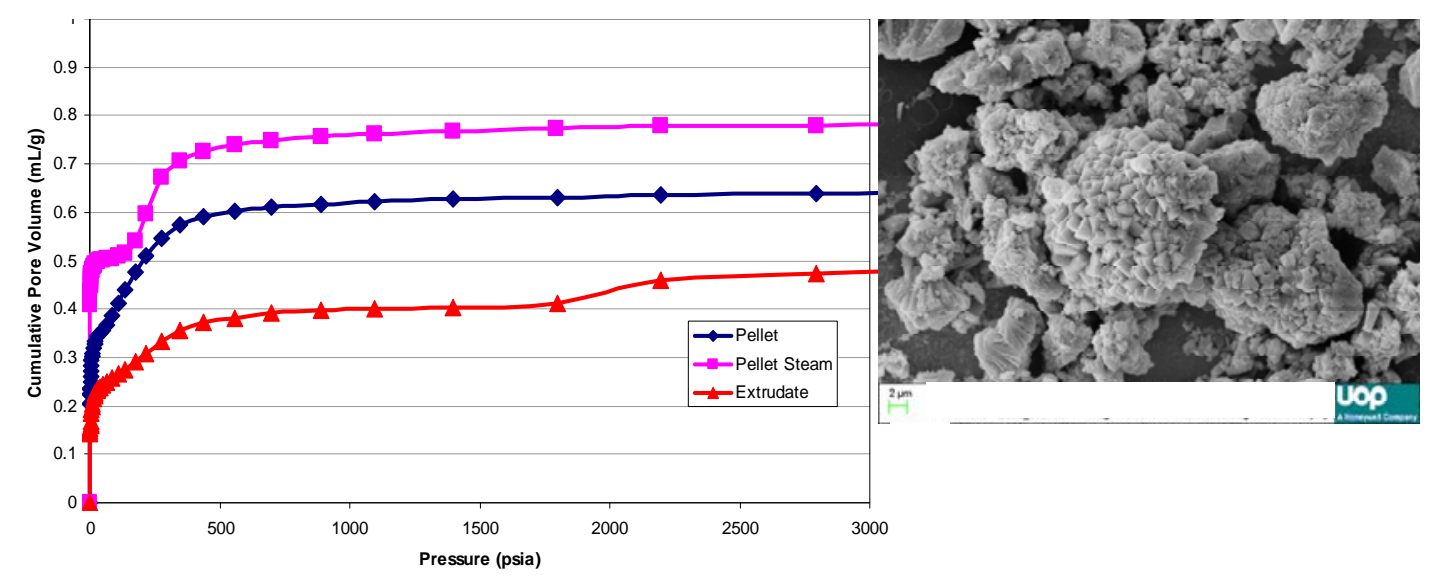

Figure 6. Mercury porosimetry on formed Ni/DOBDC samples and HRSEM image of $\mathrm{Ni} / \mathrm{DOBDC}$ powder that went into those formed materials.

HKUST-1. Cu-HKUST-1 had previously been identified as an interesting material due to its reasonable $\mathrm{CO}_{2}$ uptake capacity and hydrothermal stability. An enhancement in adsorption capacity in the presence of a small quantity of water (occupying open metal sites) was also discovered and reported in the open literature by team members (see section 3.4). Extensive solvent exchange was performed on a sample of HKUST-1 in attempt to obtain optimized surface area values reported in the literature $\left(2257 \mathrm{~m}^{2} / \mathrm{g}\right)$, but obtained Langmuir surface areas $\left(1393 \mathrm{~m}^{2} / \mathrm{g}, 150{ }^{\circ} \mathrm{C}\right.$ activation and $762 \mathrm{~m}^{2} / \mathrm{g} 200{ }^{\circ} \mathrm{C}$ activation) still fell short. A moisture study was done on the same sample, whereby it was exposed to ambient moisture in the air for two days. The result (after dentical activation conditions) was a significant decrease in surface area (809) from the original sample. This confirms that HKUST-1 is sensitive to moisture, although the XRD does not indicate degradation by any changes in peak intensity or linewidth.

Cr-MIL-101. This very high surface area material contains large pores, and has moderate thermal and hydrothermal stability. Extensive work was done towards the optimization of synthetic and activation conditions. The original procedure by Ferey and co-workers 9 involved synthesis using HF, and a cumbersome purification method of multiple filtrations and hot EtOH soaks/washing (2334/4149). A more attractive synthetic procedure by Stock and co-workers ${ }^{39}$ employed the use of $\mathrm{CH}_{3} \mathrm{COOH}$, and a purification used by Jhung and co-workers ${ }^{40}$ involving hot ethanol sonication was applied to give a high surface area Cr-MIL-101 material (2509/3943).

Ga-MIL-68. This material was predicted to have a fairly high thermal stability, with a barrier for ligand displacement of $43.7 \mathrm{kcal} / \mathrm{mol}$ (see below). Scale up was successful, but surface area was low (495/769) compared with literature values (1117/1410). Activation conditions (EtOH soaking and air-free heat treatment in an isolation chamber) were examined and were successful in increasing surface area values, 1198/1738 and $1193 / 1769$ respectively. Heat treatment unit (HTU) studies confirmed the hydrothermal stability of Ga-MIL-68 over the range $80-350{ }^{\circ} \mathrm{C}$, and $0-50 \%$ steam. 
Al-MIL-110. This material is of interest because it is formed at low $\mathrm{pH}$ and can serve as a reference material when conducting future studies of $\mathrm{pH}$ effects on metal cluster hydrolysis. Reproduction of literature conditions gave products of slightly differing composition, according to reactor size. Products from $45 \mathrm{~mL}$ reactors gave the best, highly crystalline material.

Novel MOFs from the Project team Team members from UM prepared several new series of MOF compounds during the project. ${ }^{35,41}$ An example of one of these materials is discussed here.

Reaction of a zinc salt with two linkers (BTC, benzenetricarboxylate, and T2DC, thionylthiophene dicarboxylic acid) provided crystals of UMCM-2:
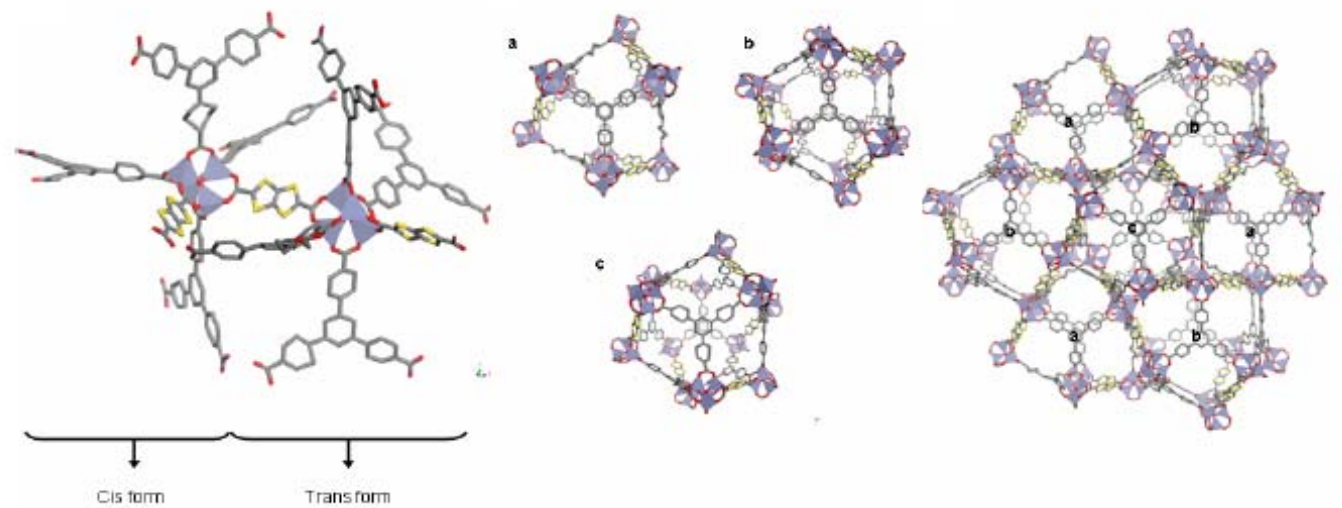

Figure 7. Structure of UMCM-2.

UMCM-2 has a surface area in excess of $4700 \mathrm{~m}^{2} / \mathrm{g}$, and it has high thermal stability as evidenced by the TGA and XRD data provided in Figure 8.

(1) TGA data of UMCM-2

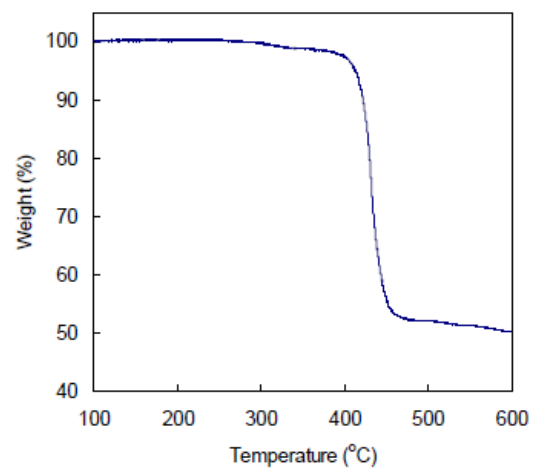

(2) Powder XRD data of UMCM-2 after heating

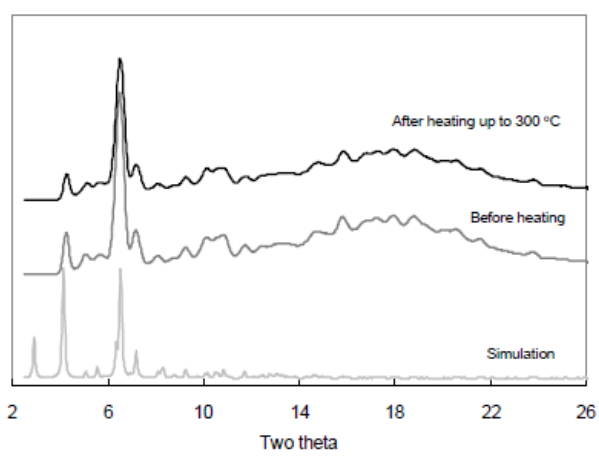

Figure 8. TGA (left) and XRD (right) analysis of UMCM-2. 
The synthesis and forming of optimized $\mathrm{Mg} / \mathrm{DOBDC}$ were scaled up to quantities sufficient for testing. Manufacturing costs are estimated to be on the order of $\$ 100 /$ pound based upon current raw material costs, experimental procedures, and product yield. Costs could be higher depending upon how the resultant product must be handled. For example, if the product must be handled exclusive of air, packaging and other costs will increase. However, opportunities for cost reduction have already been identified, and if in the future UOP decides to further pursue MOFs for $\mathrm{CO}_{2}$ capture, these issues will need to be addressed early in the next phase of the work.

Several of the most promising candidates meeting end of Phase 2 capacity and stability targets were prepared at sufficient scale to provide material for forming for further testing. Potential techniques for forming materials into commercially viable products were then investigated using these materials. The complete list of scaled-up materials is MIL-47, MIL-101, M = Zn, Ni, Co, and Mg versions of M/DOBDC, nano-IRMOF-1, HKUST-1, MIL-53, MAMS-1, La-pda, Ni-bpe, and UMCM-1. The MOFs chosen for further scale-up were the two best for $\mathrm{CO}_{2}$ capture: $\mathrm{Mg} / \mathrm{DOBDC}$ and $\mathrm{Ni} / \mathrm{DOBDC}$. Even though Ni/DOBDC was not the best performing MOF for CC, it was easier to synthesize reproducibly, and therefore this MOF was scaled-up first, near the end of Phase 2 and evaluated versus end of Phase 2 performance goals. The Ni/DOBDC scale-up was carried out at UOP. Results appear in the $\mathrm{CO}_{2}$ capacity evaluation section below.

Later, the scale-up work for $\mathrm{Mg} / \mathrm{DOBDC}$ was carried out at UM. UM researchers prepared $20 \mathrm{~g}$ of $\mathrm{Mg} / \mathrm{DOBDC}$ that was extruded, steamed and performance tested at UE, VU and UOP (results below). Recall that researchers at UOP had been struggling to make $\mathrm{Mg} / \mathrm{DOBDC}$ in high yield and/or high quality for several months, in part because UOP researchers had to devote more time and energy to other Tasks. UM researchers, originators of the $\mathrm{Mg} / \mathrm{DOBDC}$ recipe, provided the $20 \mathrm{~g}$ of required material in the final three months of the Project. This required them to change their focus from the discovery of better MOFs for $\mathrm{CO}_{2}$ adsorption to the scale-up of $\mathrm{Mg} / \mathrm{DOBDC}$.

A new synthesis for the scale-up of $\mathrm{Mg} / \mathrm{DOBDC}$ was developed in order to produce gram quantities of high quality material. The original solvothermal synthesis in a mixture of DMF:EtOH: $\mathrm{H}_{2} \mathrm{O}$ (15:1:1) consistently yielded high quality material on $\sim 100 \mathrm{mg}$ when activated. The synthesis conditions however were not amenable to direct scale-up and therefore another synthetic method has to be pursued. The current synthesis is based on a recent report by Dietzel and co-workers ${ }^{42}$ where magnesium nitrate hexahydrate is replaced by magnesium acetate tetrahydrate and the solvent system used is a 90:10 mixture of NMP: $\mathrm{H}_{2} \mathrm{O}$. We have been able to produce $\mathrm{Mg} / \mathrm{DOBDC}$ on one gram in a single batch reaction and confirm its quality through nitrogen gas sorption measurements. We have also been able to double the scale of this synthesis, but the quality of the material seems to vary from batch to batch. In some cases, an unidentified phase appears in XRD patterns (not shown). Furthermore, the amount of this phase varies between the various batches. Nitrogen gas sorption measurements on samples that possess some of this unidentified phase provided a surface area of about $600 \mathrm{~m}^{2} / \mathrm{g}$, which is roughly half the expected surface area. Some additional effort will be required to determine how to decrease this unknown phase as the synthesis is scaled up to kilogram scale. 
An optimized forming procedure was developed for Ni/DOBDC and Mg/DOBDC. Formed materials meeting or exceeding performance targets were optimized with regard to formed particle physical integrity and overall manufacturability in existing UOP equipment.

Pressing self-supporting pellets of $\mathrm{Ni} / \mathrm{DOBDC}$ is labor and time-intensive, and is not conducive to direct scale up. As such, extrusion methods utilizing simple binders like alumina were explored in order to prepare samples for scale up and characterization. Alumina was identified as the optimal additive in terms of extrusion ease and physical properties of the dried material. Mercury porosimetry results (see Figure 6) indicate that extruded MOFs have significantly higher mesoporosity than self-supporting pressed pellets. This means the extrudates should have better mass transfer than pellets. Results from VU (below) suggest capacity improvements for extrudates versus pellets as well, likely owing to better access to MOF micropores and less binder blinding.

\section{$\underline{3.2 \text { Carbon dioxide adsorption }}$}

Experimental results. The overall objective of Phase 2 was to further develop and test up to 10 MOF materials (The Project Top Ten) and demonstrate one or more MOF materials with improved performance and stability that are suitable for optimization and scale-up in Phase 3. See Table 2 for the final Project Top Ten MOFs.

Table 2. Summary of selected $\mathrm{CO}_{2}$ adsorption capacities at $311 \mathrm{~K}$ and $0.1 \mathrm{~atm}$. Overall Project $\mathrm{CO}_{2}$ capacity goal is $15 \mathrm{wt} \% \mathrm{CO}_{2}$ loading at $298 \mathrm{~K}$ and $1 \mathrm{~atm}$. The table also lists heats of adsorption for the samples at the lowest reported loadings, or an average of several loadings.

\begin{tabular}{|l|c|c|c|c|c|}
\hline sample & year & $\begin{array}{c}\text { loading } \\
\text { (mol/kg) }\end{array}$ & $\begin{array}{c}\text { loading } \\
\mathbf{( w t / )}\end{array}$ & $\begin{array}{c}\text { Heat of adsorption } \\
\text { (kJ /mol) }\end{array}$ & n (mol) \\
\hline $\mathrm{Mg} / \mathrm{DOBDC}$ & 2010 & 4.93 & 21.7 & 51.6 & average \\
\hline $\mathrm{Mg} / \mathrm{DOBDC}$ pellet & 2010 & 4.86 & 21.4 & 45.9 & average \\
\hline $\mathrm{Mg} / \mathrm{DOBDC}$ & 2009 & 4.73 & 20.8 & $62.6^{*}$ & 4 \\
\hline 5A powder & 2008 & 3.75 & 16.5 & 43.9 & 3 \\
\hline Ni/DOBDC 2009 & 2009 & 3.40 & 15.0 & 41.2 & 2 \\
\hline $\mathrm{Ni} / \mathrm{DOBDC}$ extrudate & 2009 & 2.98 & 13.1 & 43 & 2 \\
\hline 5A pellet & 2008 & 2.50 & 11.0 & 38.7 & 4 \\
\hline $\begin{array}{l}\text { Mg/DOBDC powder, } \\
\text { steamed }\end{array}$ & 2010 & 2.41 & 10.6 & 28.3 & average \\
\hline $\begin{array}{l}\text { Mg/DOBDC pellet, } \\
\text { steamed }\end{array}$ & 2010 & 1.90 & 8.4 & 26.5 & average \\
\hline Co/DOBDC & 2008 & 1.80 & 7.9 & 19.9 & 2 \\
\hline
\end{tabular}




\section{Measuring $\mathrm{CO}_{2} \underline{\text { adsorption }}$}

VU used a Rubotherm gravimetric system shown schematically in Figure 2 to measure pure $\mathrm{CO}_{2}$ isotherms for different MOFs. All samples were regenerated before isotherm measurements.

As an example, $\mathrm{CO}_{2}$ isotherms at three different temperatures for $\mathrm{Mg} / \mathrm{DOBDC}$ powder are displayed in Figure 9. The $\mathrm{CO}_{2}$ adsorption capacity at the point of interest (POI) is $4.93 \mathrm{~mol} / \mathrm{kg}$. Isosteres were obtained from points interpolated from Figure 9 and are displayed in Figure 10. The heats of adsorption at different loadings are summarized in Table 3. The average heat of adsorption is $51.6 \mathrm{~kJ} / \mathrm{mol}$.

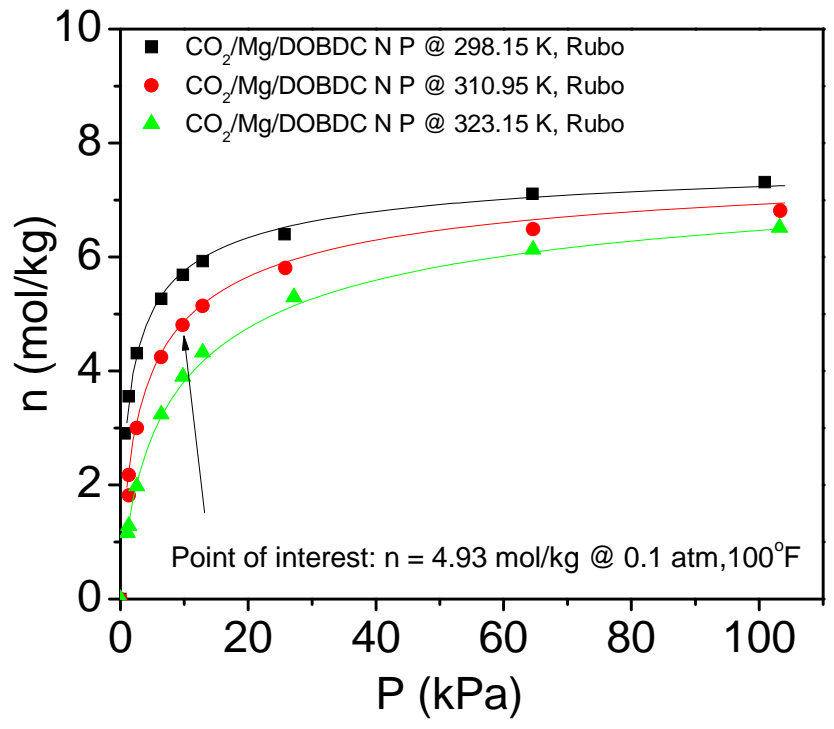

Figure 9. Gravimetric $\mathrm{CO}_{2}$ capacities for $\mathrm{Mg} / \mathrm{DOBDC}$ powder at different temperatures. Data points are shown. Curves are multi-temperature Toth equation fits.

Table 3. Heats of adsorption for the samples at different loadings

\begin{tabular}{|c|c|c|c|}
\hline Samples & Loadings (mol $/ \mathrm{kg})$ & & \\
\hline $\begin{array}{l}\mathrm{Mg} / \mathrm{DOBDC} \\
\text { powder }\end{array}$ & $\begin{array}{ll}\mathrm{n}=4.0 & \mathrm{n}=5.0 \\
53.24 \mathrm{~kJ} / \mathrm{mol} & 52.29 \mathrm{~kJ} / \mathrm{mol}\end{array}$ & $\begin{array}{l}\mathrm{n}=6.0 \\
49.33 \mathrm{~kJ} / \mathrm{mol}\end{array}$ & $\begin{array}{l}\text { Average } \\
\sim 51.6 \mathrm{~kJ} / \mathrm{mol}\end{array}$ \\
\hline
\end{tabular}

The $\mathrm{CO}_{2}$ capacity at the $\mathrm{POI}$ for $\mathrm{Mg} / \mathrm{DOBDC}$ powder is large, which can be ascribed to the unsaturated metal centers (UMCs) in its crystal structure. ${ }^{30}$ The strong interactions between the $\mathrm{CO}_{2}$ molecules and the UMCs is consistent with the large isosteric heat of adsorption. 


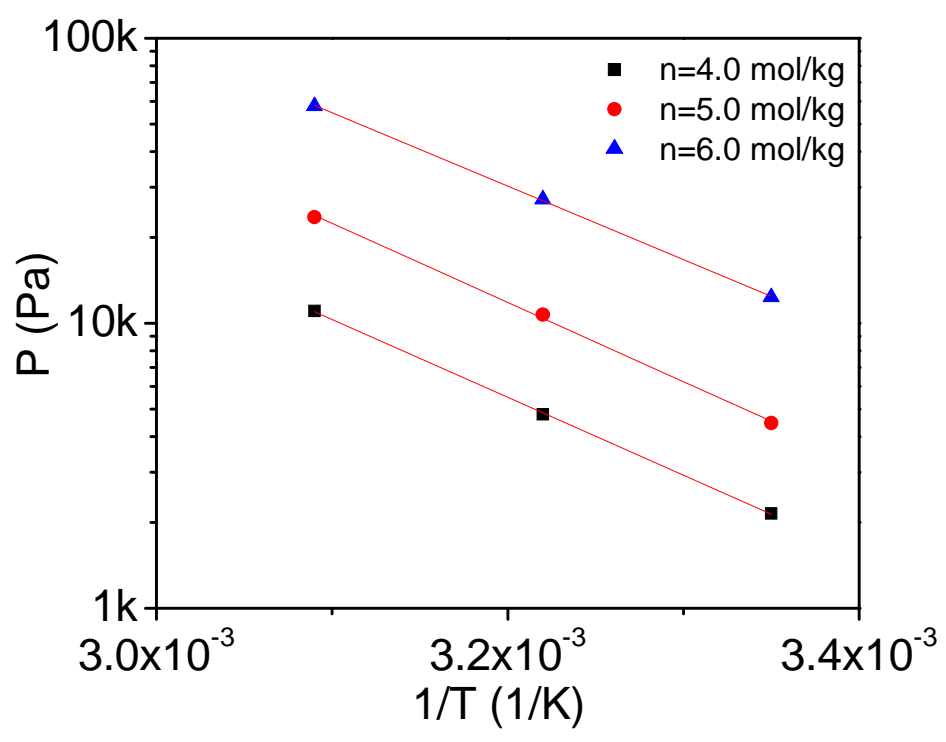

Figure 10. Isosteres of $\mathrm{CO}_{2}$ adsorption by $\mathrm{Mg} / \mathrm{DOBDC}$ powder 2010 at different temperatures. Points have been interpolated from Figure 9, and the lines are linear fits.

Following this procedure, we measured $\mathrm{CO}_{2}$ isotherms at three different temperatures for all other MOF candidates. The results, including some results for zeolites, are summarized in a bar graph shown in Figure 11. The MOF samples are sorted in decreasing order of their $\mathrm{CO}_{2}$ capacities at the POI. We did not find any general correlation between the sequence of $\mathrm{CO}_{2}$ capacities at the POI for the MOF samples and their surface areas or pore volumes. The average isosteric heats of adsorption that were determined from the isotherm data are summarized in Figure 12. The $\mathrm{CO}_{2}$ capacities at the POI and the average heats of adsorption for the MOFs samples are found to follow a trend similar to the isotherm capacities with some exceptions such as Ni-bpe. 


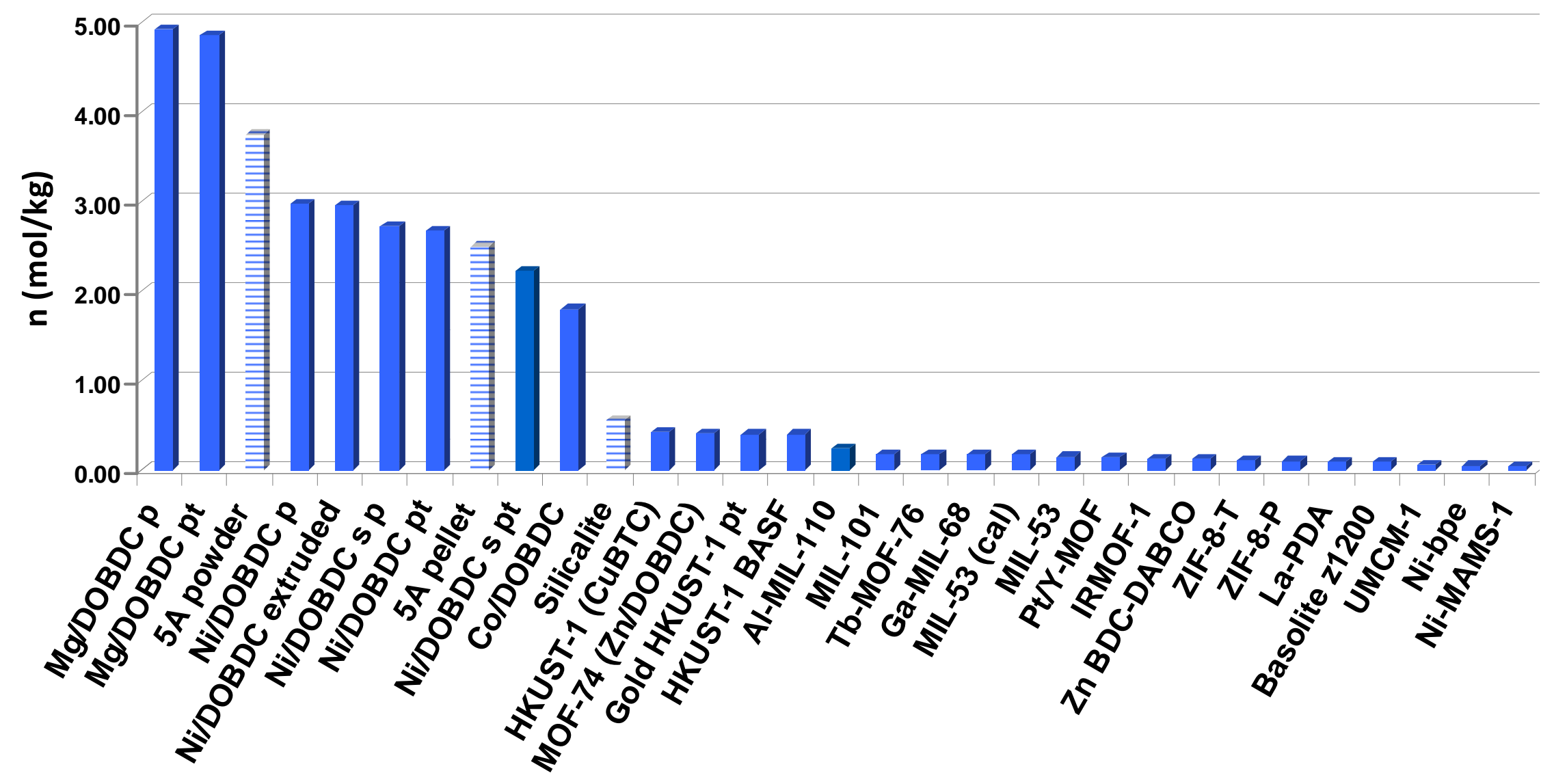

Figure 11. Summary of the $\mathrm{CO}_{2}$ capacities at the POI for all samples that we have considered. (s denotes steamed; $\mathrm{p}$ denotes powder; $\mathrm{s} \mathrm{p}$ denotes steamed power; $\mathrm{s}$ pt denotes steamed pellet.) 


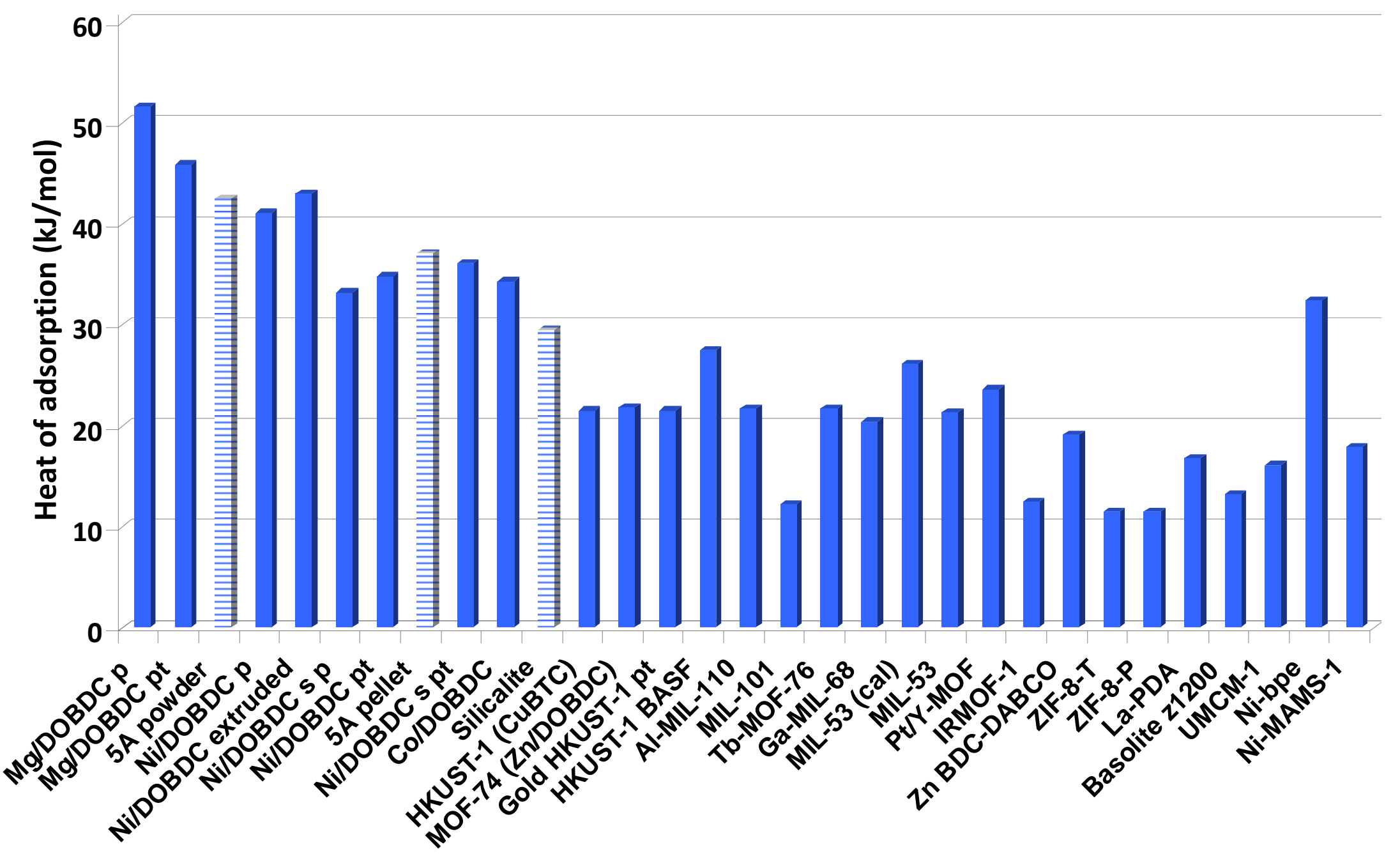

Figure 12. Summary of the average isosteric heats of adsorption for all samples that we have considered. (s denotes steamed; $\mathrm{p}$ denotes powder; $\mathrm{s} \mathrm{p}$ denotes steamed power; $\mathrm{s}$ pt denotes steamed pellet.) 
Team members at NU used molecular simulation to screen out the best MOF candidates for $\mathrm{CO}_{2}$ adsorption in the low pressure region. ${ }^{43}$ As expected, UMCs play a key role in $\mathrm{CO}_{2}$ adsorption due to coordination interactions between $\mathrm{CO}_{2}$ molecules and the UMCs. The DOBDC series of MOFs with UMCs are found to be the best MOF candidates for $\mathrm{CO}_{2}$ adsorption at low pressure. As mentioned above, metal substitution in the DOBDC series can impact the $\mathrm{CO}_{2}$ capacity significantly. This effect may be caused by the ionic character of the metal-oxide bond. Beyond the DOBDC series of MOFs, HKUST-1 (also known as $\mathrm{Cu}-\mathrm{BTC}$ and MOF-199) has the highest $\mathrm{CO}_{2}$ capacity at the POI among all other MOFs that were considered, and it is also among the most studied MOFs. This helps to explain why we selected two of the DOBDC series MOFs, Ni/DOBDC, $\mathrm{Mg} / \mathrm{DOBDC}$, and HKUST-1 as our targets for further study on $\mathrm{H}_{2} \mathrm{O}$ and simulated flue gas conditioning effects in the following sections.

At UE, a Zero-length column (ZLC) apparatus was used to evaluate $\mathrm{CO}_{2}$ adsorption on MOFs. For the ZLC experiment, the system is in flow, therefore regeneration is typically carried out overnight at the set regeneration temperature in He flow. The temperature is ramped at a rate of $1{ }^{\circ} \mathrm{C} / \mathrm{min}$ from room temperature to $110^{\circ} \mathrm{C}$, held for 2 hours and then ramped again at a rate of $1{ }^{\circ} \mathrm{C} / \mathrm{min}$ to the final regeneration temperature. When the mass spectrometer (MS) is connected to the system, we can also monitor the water concentration during the regeneration. Figure 13 shows an example of the regeneration of $\mathrm{Ni} / \mathrm{DOBDC}$.

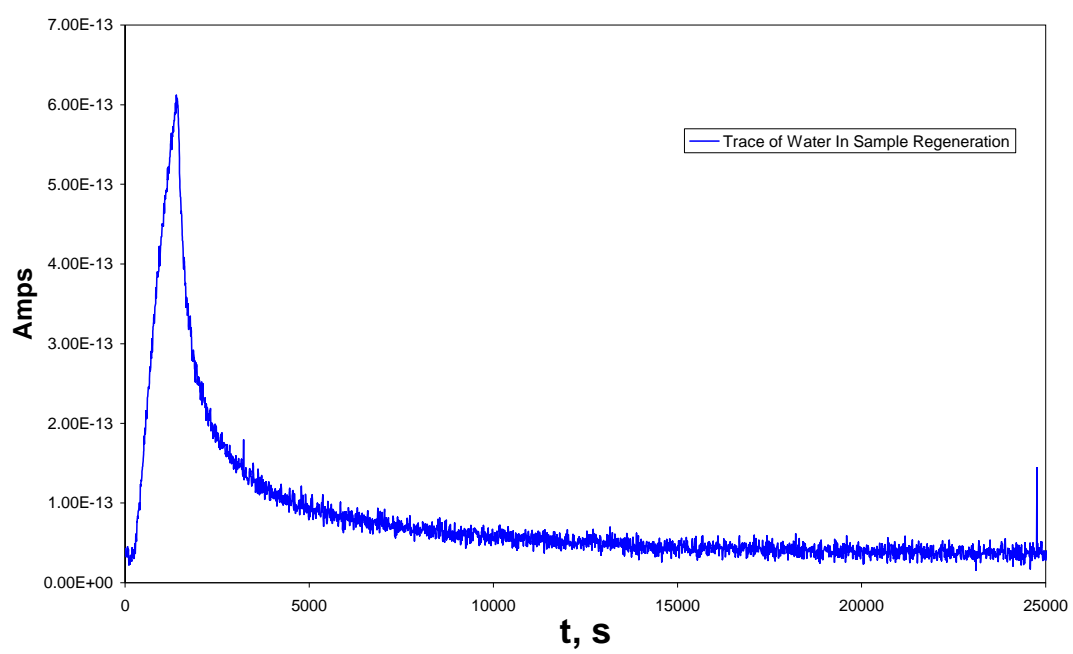

Figure 13. Water signal during regeneration of Ni/DOBDC sample.

Figure 14 shows the final ZLC ranking plots at the reference temperature of $38{ }^{\circ} \mathrm{C}(100$ $\left.{ }^{\circ} \mathrm{F}\right)$. The $\mathrm{CO}_{2}$ capacity is proportional to the area under the curves shown in the figure. Therefore the order of capacities is $\mathrm{Mg} / \mathrm{DOBDC}>\mathrm{Ni} / \mathrm{DOBDC}>5 \mathrm{~A}$ zeolite $>$ $\mathrm{Co} / \mathrm{DOBDC}$, and the order observed in the ZLC experiments confirmed the gravimetric results obtained by team members at VU.

The ZLC plots can be used also to gain insights on qualitative differences between the MOFs and 5A zeolite. Figure 14 clearly shows that for 5A there is a very significant 
proportion of the capacity at low gas phase concentrations. This means that the adsorption isotherm for $\mathrm{CO}_{2}$ on $5 \mathrm{~A}$ is much more non-linear than those for the MOFs and this has important implications for pressure swing adsorption processes where isotherm non-linearity decreases the efficiency of the regeneration and thus the overall separation performance.

Figure 15 shows the isotherms for the $\mathrm{Mg} / \mathrm{MOF}$ sample obtained using the mass balance reported in the basic theory section.

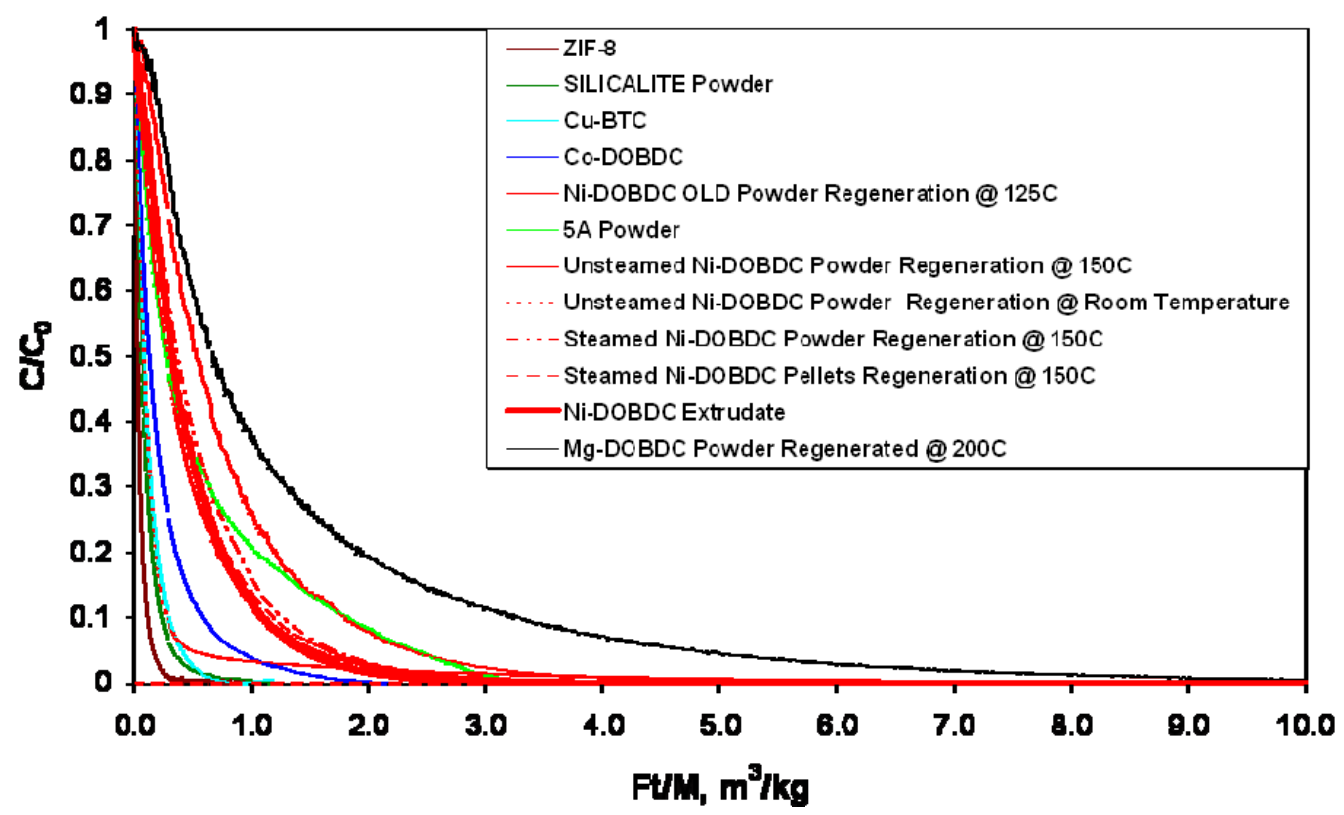

Figure 14. ZLC ranking plots.

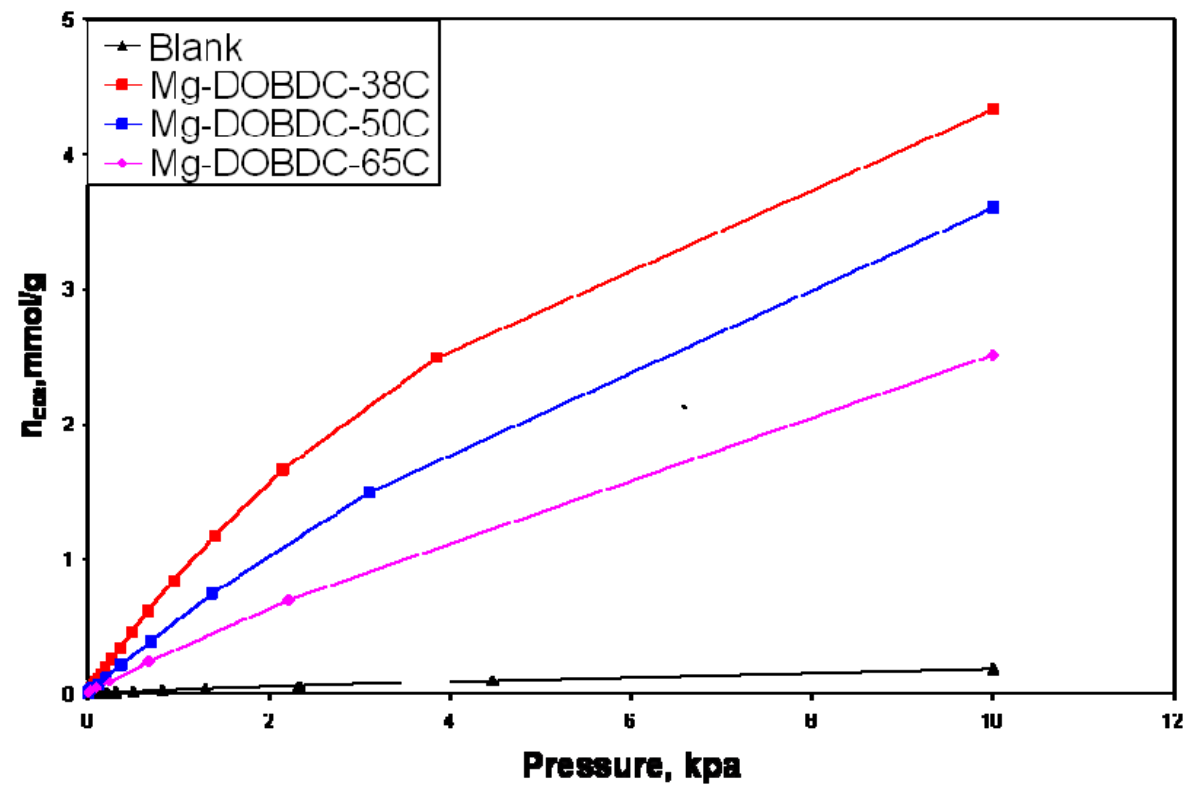

Figure 15. Isotherms for $\mathrm{Mg} / \mathrm{DOBDC}$ at different temperatures. 


\section{Modeling $\mathrm{CO}_{2} \underline{\text { adsorption }}$}

The objectives of this portion of the project were to

- Develop a model to calculate adsorption isotherms and heats of adsorption for $\mathrm{CO}_{2}$ and other small molecules in MOFs

- Use the model to understand the inflection observed in experimental $\mathrm{CO}_{2}$ adsorption isotherms in IRMOFs

- Use the model to evaluate MOFs for separations involving $\mathrm{CO}_{2}$.

Model Development and Initial Insights. One of the objectives of this program was to develop molecular modeling techniques to predict isotherms for the components of flue gas on MOFs. The major components of these streams are $\mathrm{CO}_{2}, \mathrm{~N}_{2}, \mathrm{CH}_{4}, \mathrm{H}_{2}$, and $\mathrm{H}_{2} \mathrm{O}$. Water is quite polar, and $\mathrm{CO}_{2}$ and $\mathrm{N}_{2}$ both have significant quadrupole moments. Therefore the interactions of the charge distribution of the MOFs and the sorbate molecules have a significant impact on the selectivity and capacity for $\mathrm{CO}_{2}$ and $\mathrm{N}_{2}$. In the model that we developed, the MOFs and adsorbate molecules are represented in atomistic detail, placing Lennard-Jones sites and partial charges on all atoms. Careful selection of the sorbate/sorbate potential parameters is essential, and in previous work it was found that choosing a model that works well for vapor-liquid equilibria is a good strategy. Therefore Lennard-Jones parameters and partial charges from the TraPPE force field for $\mathrm{CO}_{2}, \mathrm{~N}_{2}$, and methane have been utilized. ${ }^{45}$ Lennard-Jones parameters for the MOF atoms were taken from the DREIDING force field. ${ }^{44}$ Previously we found that DREIDING worked well for predicting adsorption isotherms of methane and hydrogen in MOFs. ${ }^{46,47}$ During the project, partial charges were calculated for the framework atoms of a variety of MOFs using density functional theory (DFT) calculations on small pieces of the MOF structures.

To test the model, adsorption isotherms for $\mathrm{CO}_{2}$ were calculated for a set of test cases using grand canonical Monte Carlo (GCMC) simulations. ${ }^{48}$ As shown in Figure 16, the predicted $\mathrm{CO}_{2}$ isotherms match quite well with the experimental isotherms for IRMOF-1, IRMOF-3, and IRMOF-6. Results also matched experiment well for MOF-177 (not shown). In addition, the simulations capture the complex temperature dependence of the isotherms essentially quantitatively, as shown in Figure 17. It should be noted that the simulations use the same parameters for all cases; the parameters are not fit to match the data.

The model has also given us some insight into the unusual inflections and steps in the isotherms. First, by running the simulations with only Lennard-Jones interactions but no Coulombic interactions, we determined that $\mathrm{CO}_{2} / \mathrm{CO}_{2}$ Coulombic interactions are critical for predicting the inflection. (See Figure 16a.) An isotherm of this shape generally indicates that sorbate/sorbate interactions are very strong compared to sorbate/sorbent interactions, which usually dominate adsorption. The strong sorbate/sorbate interactions are due to the dipole moment of $\mathrm{CO}_{2}$. We also see that the heat of adsorption increases with increasing loading in the pores. Again this is opposite to many adsorption systems and indicates strong sorbate/sorbate interactions. 

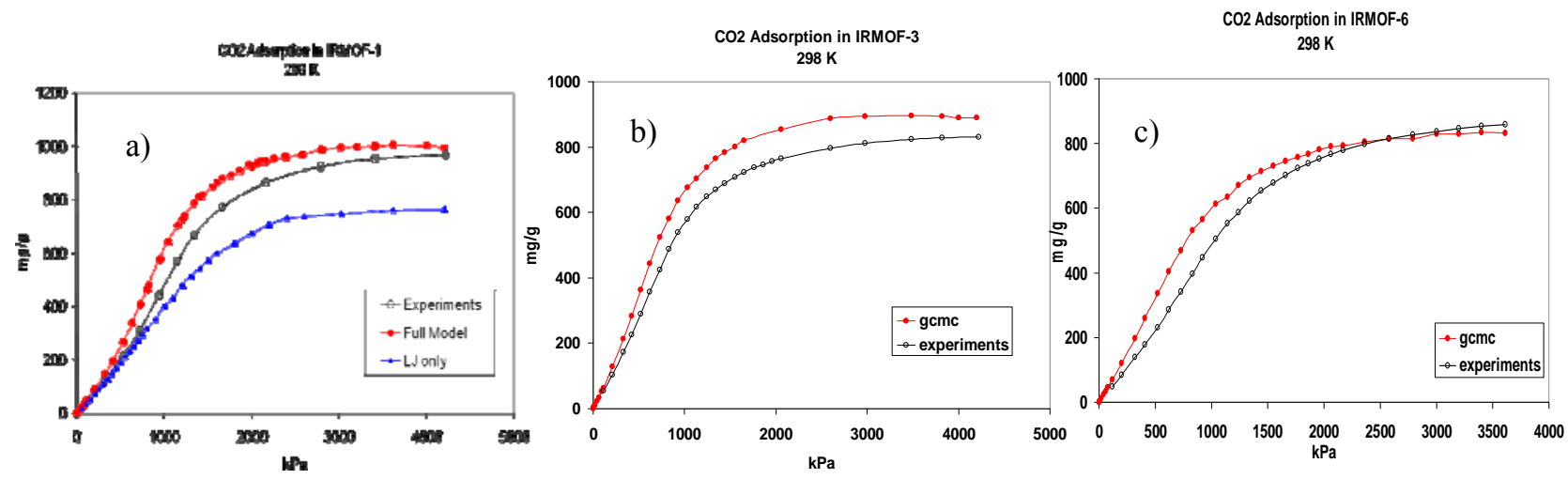

Figure 16. Comparison of simulated and experimental adsorption isotherms for $\mathrm{CO}_{2}$ at $298 \mathrm{~K}$ in a) IRMOF-1, b) IRMOF-3, and c) IRMOF-6. For IRMOF-1, the isotherm in blue includes only Lennard-Jones interactions. The simulation with only Lennard-Jones interactions does not capture the the inflection observed in the experimental isotherm, but the full model (in red) that also includes the Coulombic interactions successfully captures the shape of the isotherm.

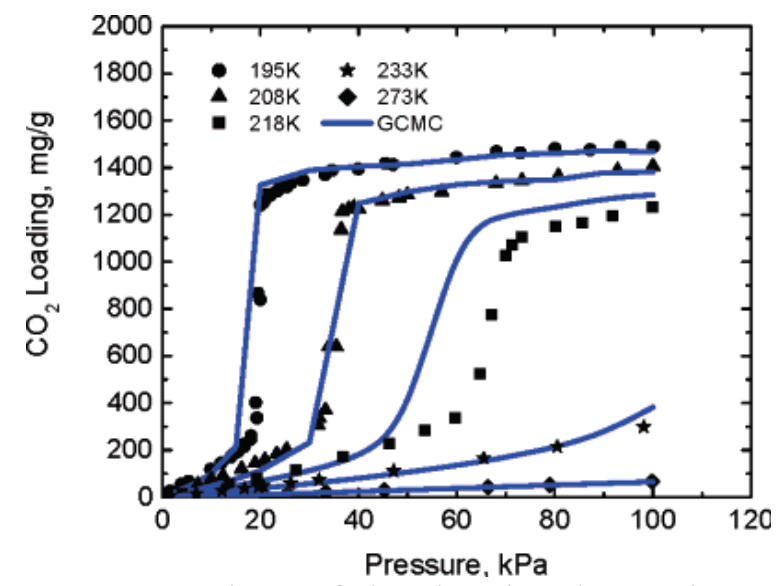

Figure 17. Comparison of simulated and experimental adsorption isotherms for $\mathrm{CO}_{2}$ in IRMOF-1 at various temperatures. ${ }^{48}$

To study the effect of linker functionality on $\mathrm{CO}_{2}$ adsorption, isotherms in IRMOF-1, 3, and 6 are compared in Figure 18. IRMOF-1 has a benzenedicarboxylate linker with no functional groups attached, whereas IRMOF-3 and IRMOF-6 have amino and alkyl functionalized linkers, respectively. IRMOF-1 has the highest saturation capacity and IRMOF-6 has the lowest. These results can be explained on the basis of the free volume of the frameworks. IRMOFs-3 and -6 have reduced pore volumes due to the amine and cyclobutane groups that protrude into the cavities. These groups, however, promote adsorption at low pressures, where IRMOF-3 and IRMOF-6 show higher adsorption than IRMOF-1. 


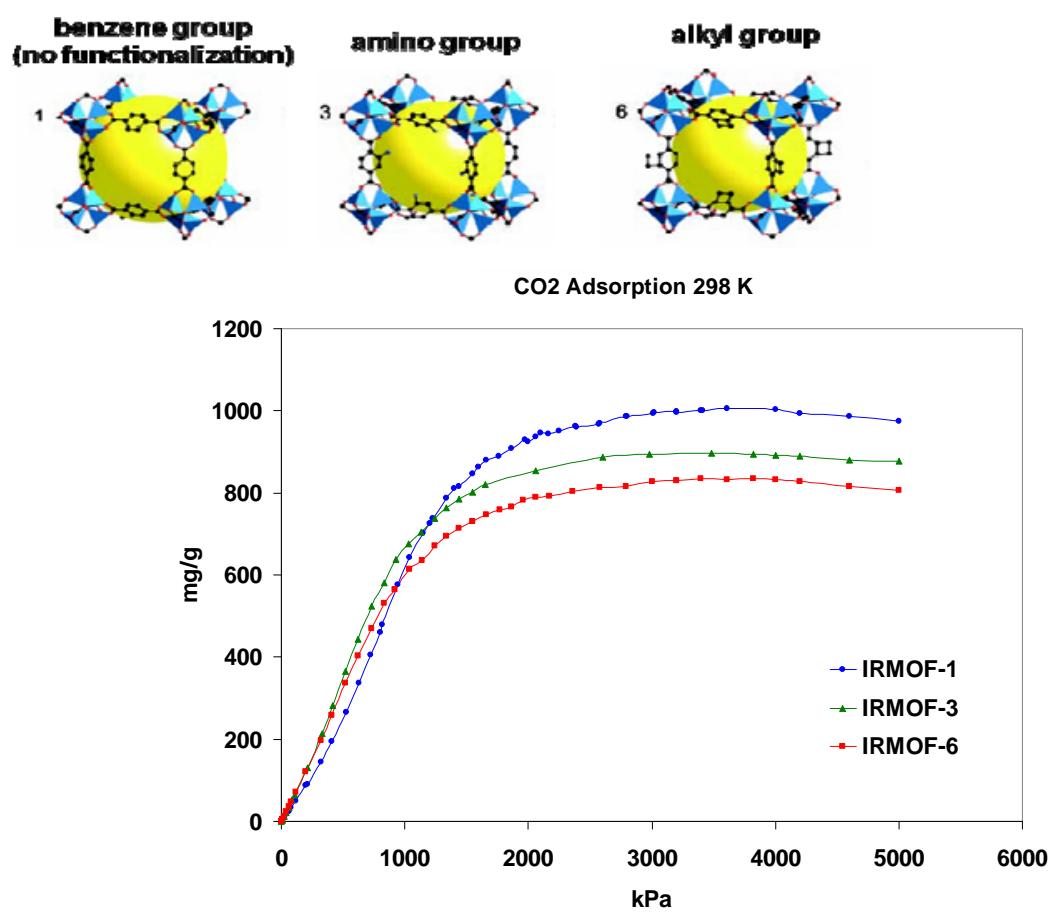

Figure 18. Comparison of simulated adsorption isotherms for $\mathrm{CO}_{2}$ in IRMOF-1 (left structure, top), IRMOF-3 (middle structure, top), and IRMOF-6 (right structure, top) at $298 \mathrm{~K}$.

Analysis of the $\mathrm{CO}_{2}$ positions from the simulations reveals that below the sharp rise in the isotherms of Figure 17, molecules are mostly adsorbed near the corners of the MOF cavities. With increasing pressure, the pores fill, leading to a type V isotherm. Type V isotherms are relatively rare and are often accompanied by hysteresis. However, neither the experiments nor the simulations display hysteresis. To shed more light on the pore filling, we plotted the density of $\mathrm{CO}_{2}$ within the pore volume of IRMOFs-1, -10, and -16 at $298 \mathrm{~K}$ (Figure 19). ${ }^{48}$ IRMOF-10 and IRMOF-16 possess approximately double and triple the pore volume per gram of IRMOF-1. The bulk density of $\mathrm{CO}_{2}$ is also shown in the figure. From these results, we find that the pressure of the pore filling shifts toward the bulk condensation pressure with increasing pore size (IRMOFs-1, -10, -16).

Model Validation. To test the model on a wider range of MOFs, we selected a diverse collection of 14 MOFs shown in Table $4 . \mathrm{CO}_{2}$ isotherms at $298 \mathrm{~K}$ were predicted using the strategy described above and compared with experimental data from the literature or obtained as part of this project. ${ }^{43}$ The simulated uptake at 0.167 bar and $298 \mathrm{~K}$ is compared with experiments in Table 4. The ranking of the materials (shown in square brackets) from simulation is in very good agreement with that from experiment. Note that seven of the 14 MOFs have open metal sites (the 4 versions of MOF-74, HKUST-1, UMCM-150, and UMCM-150(N)2). The simple model used here, which does not account for polarization or orbital interactions, is expected to underpredict $\mathrm{CO}_{2}$ adsorption in MOFs with open metal sites, and this is indeed observed (Figure 20). Such correlations were also reported for $\mathrm{CO}_{2}$ adsorption in MOFs. ${ }^{43}$ Figure 20 shows that, for the MOFs studied here, there is an excellent correlation between the $\mathrm{CO}_{2}$ uptake and heat 
of adsorption at $P<1$ bar. Also, there is no correlation with the surface area or the free volume. An interesting exception to the trend in Figure 20 is the $\operatorname{Pd}(2-p y m o)_{2} \mathrm{MOF}$, which has a lower free volume and smaller pores than those of other materials. Thus, even at low pressures, the pores are essentially filled, shifting this MOF into the free volume regime.
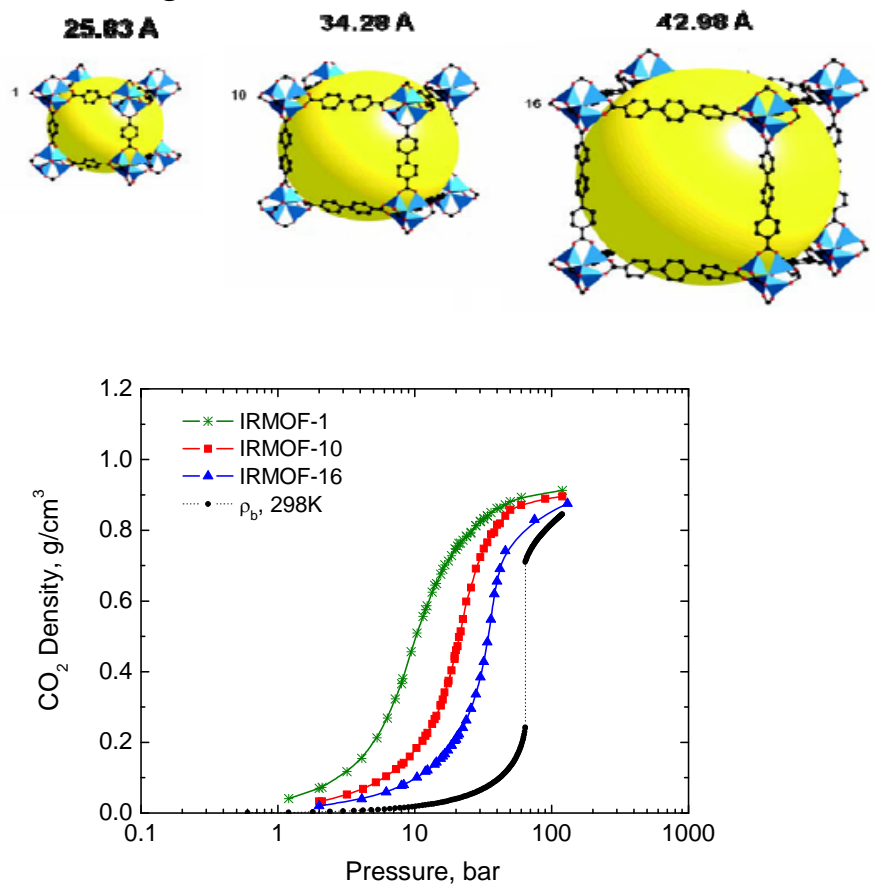

Figure 19. Density of $\mathrm{CO}_{2}$ in IRMOF-1 (left structure, top), IRMOF-10 (middle structure, top), and IRMOF-16 (right structure, top) compared with bulk values at $298 \mathrm{~K}$. The adsorbed phase densities are calculated considering only the free volumes of the crystal structures. $^{48}$

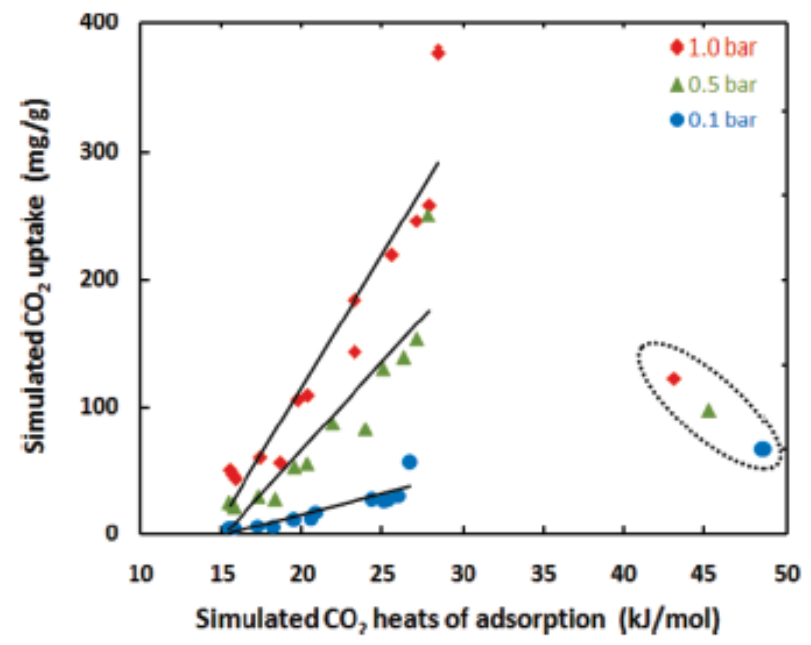

Figure 20. $\mathrm{CO}_{2}$ uptake and heats of adsorption for the screened MOFs at 0.1, 0.5, and 1 bar from simulation. Data were obtained at 293-298 K (SI). The points in the dotted circle are for $\operatorname{Pd}(2-\text { pymo })_{2}$. 


\begin{tabular}{|l|l|l|}
\hline \multirow{2}{*}{ MOF } & \multicolumn{2}{|l|}{$\mathrm{CO}_{2}$ Adsorption (mg/g) } \\
\cline { 2 - 3 } & Experiment & Simulation \\
\hline Mg-MOF-74 & $286.6[1]$ & $87.3[1]$ \\
\hline Ni-MOF-74 & $206.5[2]$ & $47.5[3]$ \\
\hline Co-MOF-74 & $170.6[3]$ & $43.0[4]$ \\
\hline Zn-MOF-74 & $86.2[4]$ & $42.7[5]$ \\
\hline Pd (2-pymo) 2 & $45.8[5]$ & $62.3[2]$ \\
\hline HKUST-1 & $43.4[6]$ & $33.0[6]$ \\
\hline UMCM-150 & $21.4[7]$ & $21.7[7]$ \\
\hline UMCM- & $20.9[8]$ & $16.7[9]$ \\
\hline 150(N) 2 & $13.3[9]$ & $20.5[8]$ \\
\hline MIL-47 & $8.3[10]$ & $9.1[11]$ \\
\hline ZIF-8 & $8.2[11]$ & $9.8[10]$ \\
\hline IRMOF-3 & $6.9[12]$ & $8.5[12]$ \\
\hline MOF-177 & $6.5[13]$ & $7.7[13]$ \\
\hline IRMOF-1 & $5.7[14]$ & $8.5[12]$ \\
\hline UMCM-1 & &
\end{tabular}

Table 4: Single-component $\mathrm{CO}_{2}$ adsorption at 0.167 bar from experiment and simulation. The numbers in brackets indicate the MOF ranking for each column based on the amount of adsorbed $\mathrm{CO}_{2}$. The temperature for the experimental and simulation adsorption data points is $298 \mathrm{~K}$, except for HKUST-1 and $\operatorname{Pd}(2 \text {-pymo })_{2}$, where the experimental temperatures were $295 \mathrm{~K}$ and $293 \mathrm{~K}$, respectively.

Nevertheless, the model selects the top five MOFs correctly, and the amount of $\mathrm{CO}_{2}$ adsorbed in the MOFs without open metal sites is in very good agreement with experiment. Identifying the best candidates is the most important task in screening, and the model is quite successful by this standard. Once the top candidates are identified, they can be studied in more detail computationally and experimentally. Given this validation, the model could be used for ranking other materials, providing insights and suggesting the most promising materials for experimental study. Note that new and even hypothetical MOFs can easily be screened using modeling.

Extension to Mixtures. In other work, we have shown that we can predict adsorption of methane, ${ }^{46}$ hydrogen, ${ }^{47}$ and nitrogen in MOFs in good agreement with experiment. Measuring multicomponent adsorption is tedious and time consuming, but mixture simulations are only slightly more difficult than single-component simulations. Thus, we simulated mixtures of $\mathrm{CO}_{2}$ and $\mathrm{N}_{2}$ in the same $14 \mathrm{MOFs}$ at conditions relevant for $\mathrm{CO}_{2}$ capture from flue gas. We found that a ranking of the MOFs based on the singlecomponent $\mathrm{CO}_{2}$ uptake is very similar to the ranking based on the mixture $\mathrm{CO}_{2} / \mathrm{N}_{2}$ selectivity. ${ }^{49}$ In addition, mixture results predicted from the single-component isotherms plus ideal adsorbed solution theory (IAST) agree well with those calculated from full 
GCMC mixture simulations. The results indicate that one can use the single-component $\mathrm{CO}_{2}$ isotherms to screen MOFs for $\mathrm{CO}_{2}$ capture, rather than performing more timeconsuming mixture simulations or experiments.

Modeling $\mathrm{CO}_{2}$ adsorption summary. A virtual high-throughput screening model was developed and used to screen MOFs for removal of $\mathrm{CO}_{2}$ from flue gas. The model was validated against experimental adsorption isotherms for a diverse set of 14 MOFs. Examining this set of materials, we found that below 1 bar $\mathrm{CO}_{2}$ uptake correlates well with the enthalpy of adsorption and the best MOFs for removal of $\mathrm{CO}_{2}$ from flue gas have a high density of open metal sites. Electrostatic interactions were found to be important for proper modeling of $\mathrm{CO}_{2}$ adsorption in MOFs. Using Monte Carlo simulations we discovered that a low loading of water molecules, bound to coordinatively unsaturated metal centers, can increase $\mathrm{CO}_{2}$ adsorption significantly at low pressures in the MOF HKUST-1. This prediction was later verified experimentally, providing additional confidence in the modeling. This opens up a new method of tuning MOFs for adsorption separations and other applications. The modeling has also been extended to simulations of mixtures, especially $\mathrm{CO}_{2} / \mathrm{N}_{2}$.

\section{$\underline{3.3 \text { Kinetics of } \mathrm{CO}_{2}}$ adsorption}

\section{Concentration-swing frequency response (CSFR) method}

In our $\mathrm{CO}_{2}$ adsorption rate study, we examined mass transfer rates for three different thicknesses of HKUST-1 and Ni/DOBDC pellets. An example result for an HKUST-1 pellet is shown in Figure 21, and an example result for a Ni/DOBDC pellet is shown in Figure 22. The pellets are labeled as $\mathrm{M}-1, \mathrm{M}-2$, and $\mathrm{M}-3$ ( $\mathrm{M}=\mathrm{Cu}$ for HKUST-1 and $\mathrm{M}=\mathrm{Ni}$ for $\mathrm{Ni} / \mathrm{DOBDC}$ ) in the order of increasing thickness. Data points are from experiments, and the values of the diffusivity parameter $\eta$, defined below, were obtained by fitting the experimental data. Predicted curves are also included to verify the fitting results. The relative positions between experimental data points and the predicted curves change from one pellet to another, which indicates a dependency of the estimated diffusivity parameters on the pellet thickness and also shows good agreement between the predicted curves and fitting results. The estimated diffusivity parameters for all of the HKUST -1 and Ni/DOBDC pellets are summarized in Table 5 . The diffusivity parameters 


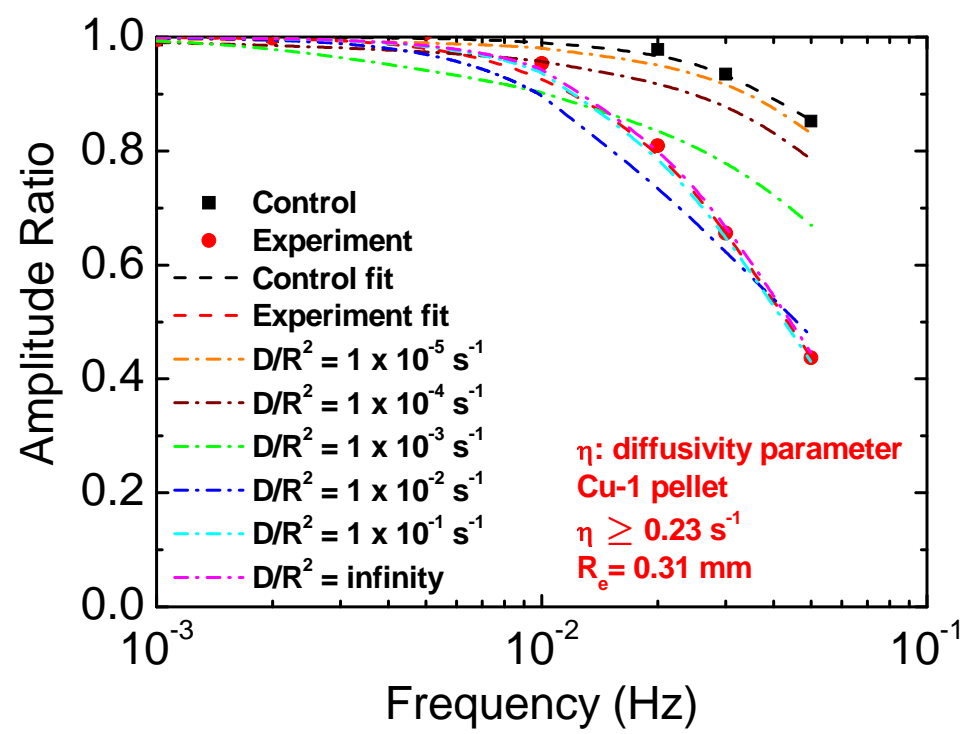

Figure 21. Frequency response results for HKUST-1 pellet-1.

shown in these figures are several orders of magnitude larger than we have found for some other adsorbents, ${ }^{50}$ which indicates very fast rates for $\mathrm{CO}_{2}$ transport in both HKUST-1 and Ni/DOBDC pellets. The dependency of the measured rates on the equivalent pellet radii suggests that the mass transfer mechanism for $\mathrm{CO}_{2}$ adsorption in HKUST-1 and Ni/DOBDC pellets is macropore diffusion control. The macropore diffusivity term $D_{\mathrm{p}} / R_{\mathrm{e}}^{2}$ and the diffusivity parameter $\eta$ that we obtain in our study are

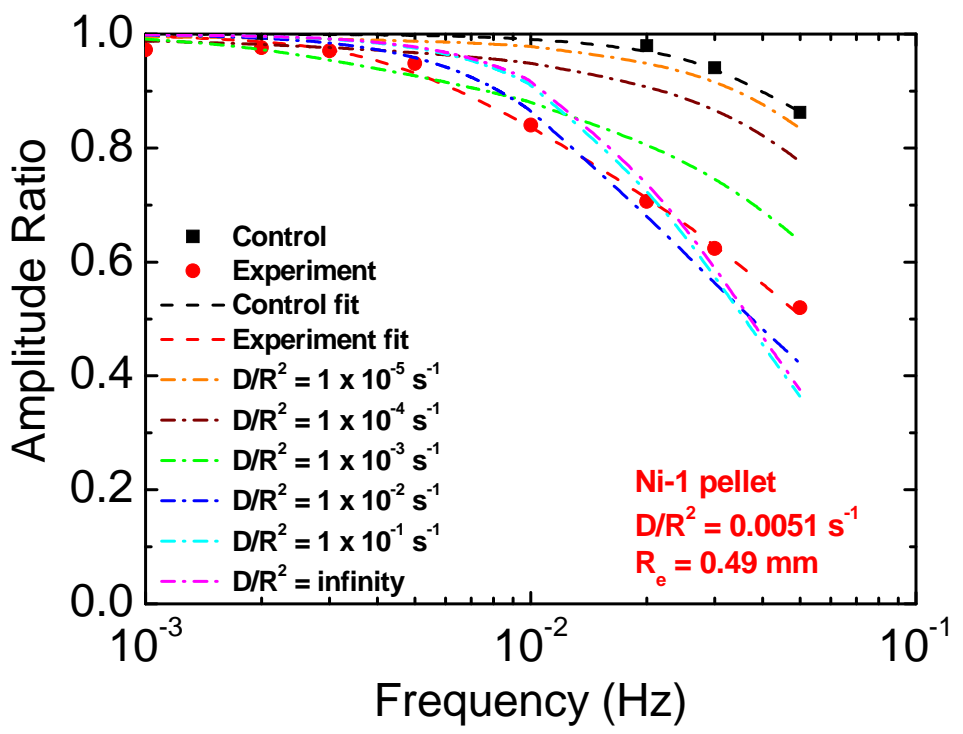

Figure 22. Frequency response results for Ni/DOBDC pellet-1. 
related by ${ }^{51}$

$$
D_{\mathrm{p}} / R_{\mathrm{e}}^{2}=\eta\left(1+\rho_{\mathrm{p}} K / \varepsilon_{\mathrm{p}}\right)
$$

where $D_{\mathrm{p}}$ is the macropore diffusivity, $\rho_{\mathrm{p}}$ is the pellet density $\left(\mathrm{kg} / \mathrm{m}^{3}\right), K$ is the local isotherm slope $\left(\mathrm{m}^{3} / \mathrm{kg}\right),{ }^{\varepsilon_{\mathrm{p}}}$ is the macropore porosity, and $R_{\mathrm{e}}$ is the equivalent radius given by ${ }^{52}$

$$
R_{\mathrm{e}}=3 \times \frac{\text { volume of pellet }}{\text { surface area of pellet }}
$$

The identification of macropore diffusion as the controlling resistance is consistent with the open structures of these two MOFs. Furthermore, if we replace the LHS of equation 1 with the bulk diffusivity term, $D_{\text {bulk }} / R_{\mathrm{e}}^{2} \tau$, where $D_{\text {bulk }}$ is the $\mathrm{CO}_{2}$ diffusivity in He and $\tau$ is the tortuosity, we obtain diffusivity parameters, $\eta$-calc, that have the same order of magnitude as the diffusivity parameters, $\eta$-exp, estimated from our experiments, as shown in Table 5. This indicates that $\mathrm{CO}_{2}$ molecules diffuse through the MOF pellets much like binary diffusion in the bulk gas phase, and the main resistance is diffusion through the intercrystal macropores. This is in contrast to a rate limitation dominated by diffusion in intracrystal micropores, which has previously been considered. ${ }^{53}$

Table 5. Summary of the diffusivity parameters for MOF pellets with different thicknesses.

\begin{tabular}{llll}
\hline sample & Equivalent radius $(\mathrm{mm})$ & $\eta$-exp $\left(\mathrm{s}^{-1}\right)$ & $\eta$-calc $\left(\mathrm{s}^{-1}\right)$ \\
\hline $\mathrm{Cu}-1$ pellet & 0.31 & $\geq 0.23$ & 0.44 \\
$\mathrm{Cu}-2$ pellet & 0.67 & 0.049 & 0.096 \\
$\mathrm{Cu}-3$ pellet & 1.11 & 0.017 & 0.035 \\
$\mathrm{Ni}-1$ pellet & 0.49 & 0.0051 & 0.0043 \\
$\mathrm{Ni}-2$ pellet & 0.57 & 0.0041 & 0.0031 \\
$\mathrm{Ni}-3$ pellet & 0.82 & 0.0019 & 0.0015 \\
\hline
\end{tabular}

\section{$\underline{\text { Zero-length column method }}$}

ZLC kinetic experiments can be carried out on both powders and pellets. The main difference is that in the case of powders the mass transfer resistance is typically due to internal diffusion in the crystals, while for pellets one can also have diffusion in the macropores. What is important is that in order to be able to measure diffusivities one has to run the ZLC under conditions that are far from equilibrium control.

Basic Theory of kinetic ZLC Experiments. We start again from the mass balance in the column, assuming that this behaves as a well mixed cell.

$V_{f} \frac{d c}{d t}+V_{s} \frac{d \bar{q}}{d t}=-(F C)_{\text {out }}$

Note that now we are using the average concentration in the solid in the second term on the RHS. 
In the case of crystals, we now need to consider the mass balance in the solid, where we assume that we have diffusion in the micropores as the mass transfer mechanism.

$\frac{\partial q}{\partial t}=D \frac{\partial^{2} q}{\partial z^{2}}$

where $\mathrm{D}$ is the diffusion coefficient, $\mathrm{m}^{2} / \mathrm{s}$, and for simplicity we assumed a slab geometry. If we apply the mass balance in the solid at the solid's surface ( $z=1$ the halfwidth of the slab

$V_{s} \frac{d \bar{q}}{d t}=\left.A_{s} D \frac{\partial q}{\partial z}\right|_{z=l}$

where $A_{s}$ is the solid surface. For a slab $V_{s} / A_{s}=1$.

Combining eqs. 3 and 5 one obtains the key dimensionless grouping in the ZLC experiment

$$
L=\frac{F l^{2}}{H V_{S} D}
$$

where $\mathrm{H}$ is the dimensionless Henry law constant, i.e. for a linear isotherm $\mathrm{q}=\mathrm{Hc}$.

The parameter $\mathrm{L}$ gives an indication of how quickly we are changing the concentration in the ZLC compared to the diffusional time constant $1^{2} / \mathrm{D}$. For equilibrium measurements we want L to be small, ideally less than 1, so we need to have small particles, large amounts of solid and low flowrates. For kinetic experiments we need to have L $>5$ in order to have an accurate measurement of the diffusivity. In this case we want to increase the flowrate, use large crystals and decrease the amount of solid in the column. In the case of pellets we need to consider the mass balance in the solid

$$
\left(1-\varepsilon_{P}\right) \frac{\partial q}{\partial t}+\varepsilon_{P} \frac{\partial c_{P}}{\partial t}=\frac{\varepsilon_{P}}{\tau} D_{m} \frac{\partial^{2} c_{P}}{\partial z^{2}}
$$

where $\mathrm{c}_{\mathrm{P}}$ is the concentration in the macropores, $\mathrm{D}_{\mathrm{m}}$ is the molecular diffusivity of $\mathrm{CO}_{2}$ in the carrier gas, $\varepsilon_{\mathrm{P}}$ is the void fraction of the pellet and $\tau$ is the tortuosity of the pellet. We could write an additional mass balance in the micropores, but for the cases that we have encountered in the project we were always under conditions where the the solid was at equilibrium with the gas in the macropores, i.e. $\mathrm{q}=\mathrm{Hc}_{\mathrm{P}}$. In this case

$$
\left[\left(1-\varepsilon_{P}\right) H+\varepsilon_{P}\right] \frac{\partial c_{P}}{\partial t}=\frac{\varepsilon_{P}}{\tau} D_{m} \frac{\partial^{2} c_{P}}{\partial z^{2}}
$$

which can be rearranged to obtain Fick's diffusion equation and the effective macropore diffusivity

$$
\frac{\partial c_{P}}{\partial t}=D_{P} \frac{\partial^{2} c_{P}}{\partial z^{2}} \quad D_{P}=\frac{\varepsilon_{P} D_{m} / \tau}{\varepsilon_{P}+\left(1-\varepsilon_{P}\right) H}
$$

Kinetic Experiments on Crystals. The largest crystals synthesised by UOP were for the $\mathrm{Co} / \mathrm{DOBDC}$ material. An SEM image is shown in Figure 23. 


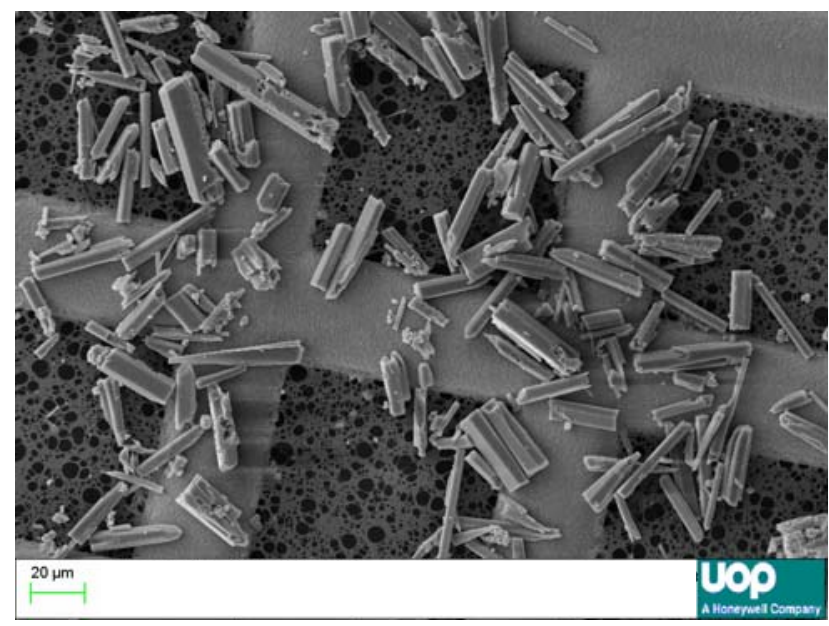

Figure 23. SEM image of Co/DOBDC crystals.

The SEM image was analysed to obtain the particle size distribution (PSD) of the crystals and this was regressed using a log-normal PSD, which shows an average size of approximately $6.5 \mu \mathrm{m}$.

ZLC experiments were carried out at two different flow rates in order to establish kinetic or equilibrium control. Figure 24 shows the results of the $\mathrm{Ft}$ plot indicating that the curves overlap and the diffusional time constant is too fast for an accurate measurement, i.e. we are still in equilibrium control even at the higher flow rate. While this is disappointing from a scientific point of view, since it would have been interesting to be able to obtain diffusion data for MOF systems, it is actually a positive result in terms of the $\mathrm{CO}_{2}$ capture process, because in an equilibrium driven process mass transfer limitations reduce the separation efficiency of the material. Based on the fact that we were not able to see a kinetic effect on these crystals, we can nevertheless conclude that the $\mathrm{L}$ parameter in the experiment was $<1$ and therefore the diffusivity was greater than $2 \times 10^{-12} \mathrm{~m}^{2} / \mathrm{s}$.

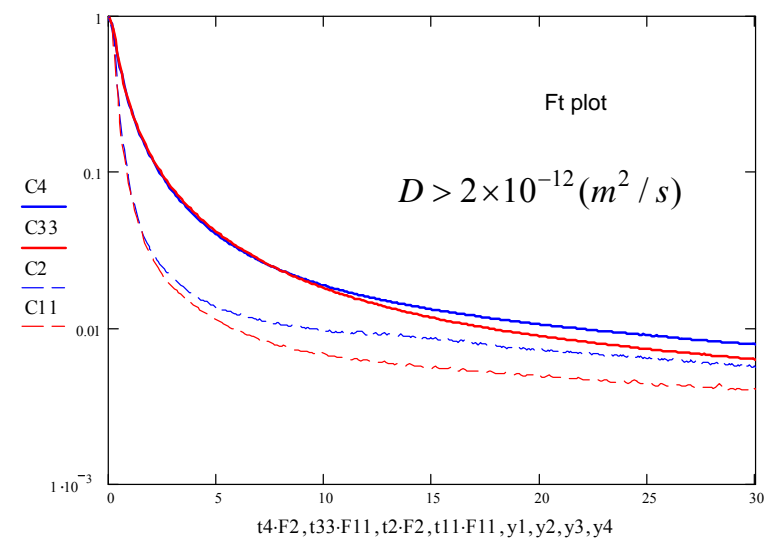

Figure 24. ZLC curves for $\mathrm{Co} / \mathrm{DOBDC}$ at 30 and $45 \mathrm{cc} / \mathrm{min}$ at $38^{\circ} \mathrm{C}$. 
The kinetic behaviour of the process is likely to be controlled by macropore diffusion in the formed (pellets) material.

ZLC kinetic experiments on pellets. Figure 25a shows the pressed Ni/DOBDC pellet as received from UOP. Since the ZLC is packed in a $1 / 8$ " Swagelok union fitting, the original pellet could not be tested directly. Fragments of different sizes were broken off the material as shown in Figures 25b, 25c and 25d. The first test was to see if these fragments showed consistent adsorption capacities. There should be very little variation between the fragments, since the pellets do not contain a binder and this is confirmed in Figure 26.

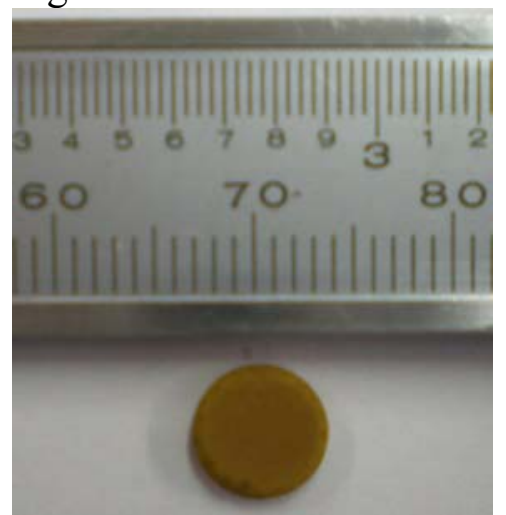

(a)

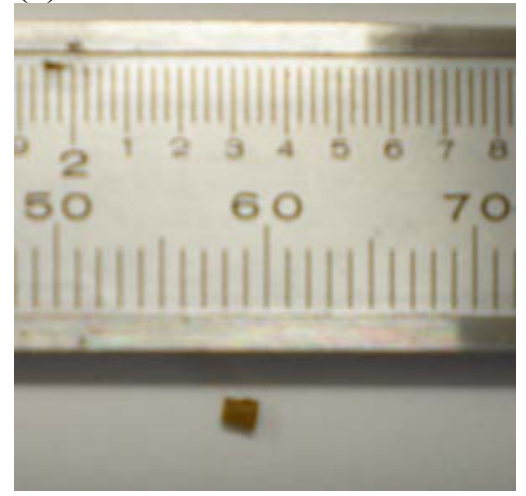

(c)

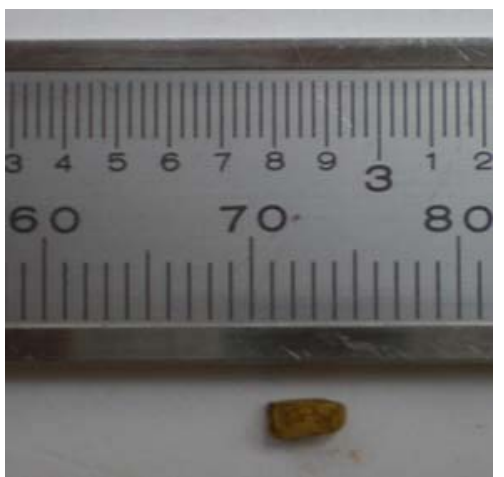

(b)

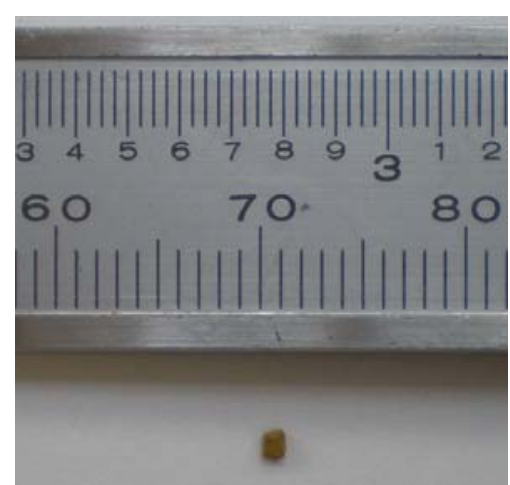

(d)

Figure 25. Formed Ni/DOBDC pellet and fragments used in the kinetic experiments.

While the fragments are more similar to 3D bodies with square or rectangular faces, for mass transfer purposes the kinetics can be approximated by the behaviour of spheres that have the same surface to volume ratio $=3 / R_{P}$. Table 6 includes the dimensions of the individual pellets and the equivalent radius calculated on this basis.

From the previous results on the crystals we have established that the micropore diffusivity in similar materials is at least $10^{-12} \mathrm{~m}^{2} / \mathrm{s}$, which for a $1 \mu \mathrm{m}$ crystal corresponds to a micropore diffusional time constant, $\mathrm{R}^{2} / \mathrm{D}$, less than 1 second. This is clearly faster than the kinetic response observed for the pellets. 


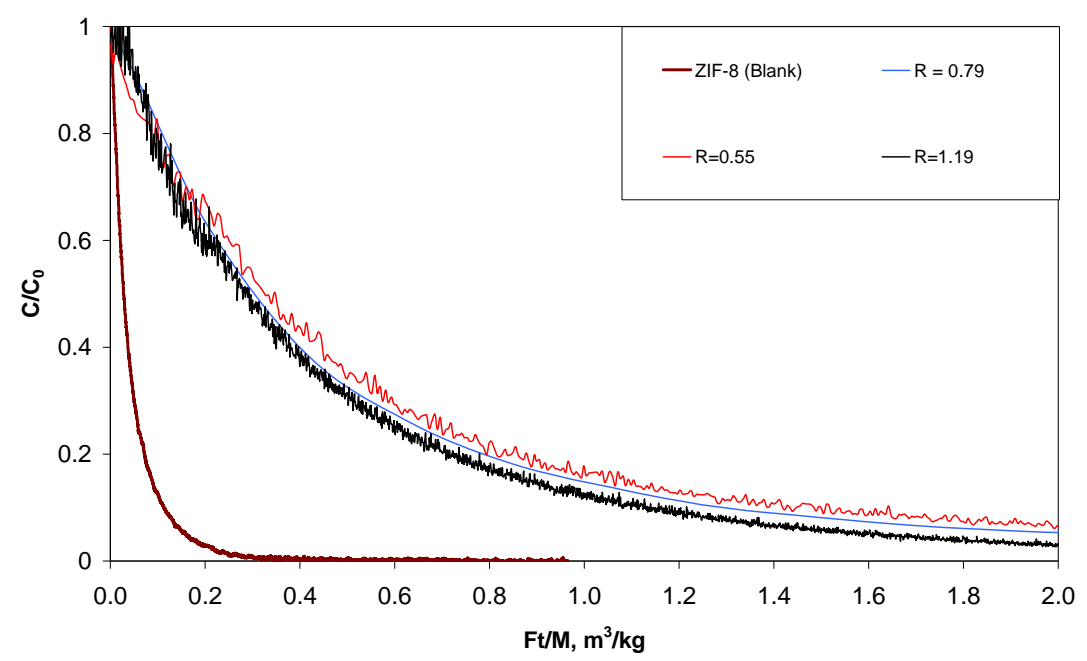

Figure 26. Comparison of adsorbent capacity of different Ni/DOBDC fragments.

Table 6. Dimensions of the fragments

\begin{tabular}{|l|l|l|l|l|l|l|l|}
\hline Material & $\begin{array}{l}\text { Mass } \\
\text { of } \\
\text { Sample } \\
(\mathrm{mg})\end{array}$ & $\begin{array}{l}\text { Width } \\
(\mathrm{mm})\end{array}$ & $\begin{array}{l}\text { Length } \\
(\mathrm{mm})\end{array}$ & $\begin{array}{l}\text { Height } \\
(\mathrm{mm})\end{array}$ & $\begin{array}{l}\text { Surface } \\
\text { Area } \\
\left(\mathrm{mm}^{2}\right)\end{array}$ & $\begin{array}{l}\text { Volume } \\
\left(\mathrm{mm}^{3}\right)\end{array}$ & $\begin{array}{l}\text { Equivalent } \\
\text { Radius } \\
(\mathrm{mm})\end{array}$ \\
\hline $5 \mathrm{~b}$ & 8.8 & 2.0 & 4.2 & 1.9 & 40.4 & 16.0 & 1.19 \\
\hline $5 \mathrm{c}$ & 2.7 & 1.8 & 2.0 & 1.2 & 16.3 & 4.3 & 0.79 \\
\hline $5 \mathrm{~d}$ & 1.5 & 1.0 & 1.4 & 1.0 & 7.6 & 1.4 & 0.55 \\
\hline
\end{tabular}

From a ZLC experiment we can obtain $D_{P} / R_{P}^{2}$ from the slope of the long time asymptote at higher flow rates. In the initial tests we used all the equilibrium and kinetic information to obtain an estimate of the macropore void fraction, $\varepsilon_{\mathrm{P}}=0.21$ (and $\tau=1 / \varepsilon_{\mathrm{P}}=$ 4.7), which is a reasonable value considering that the pellets are made from a powder with a relatively wide particle size distribution. To confirm this further, we then repeated the experiments first on the smallest pellet, but found that this was always too close to equilibrium control.

This is reasonably consistent with the fact that the diffusional time constant would be $(0.79 / 0.55)^{2}=2.1$ times faster, but for this sample the mass and volume result in a pellet density that is lower than that of the other two fragments and the dimensions may have to be measured with greater accuracy using an image analysis tool. In any case it is clear that the smallest sample is also the fastest and this confirms macropore diffusion control. We then tested the largest pellet, and the diffusional time constant allows a direct comparison between the two pellet sizes, i.e. $0.022 / 0.009=2.4$ can be compared directly 
with $(1.19 / 0.79)^{2}=2.3$. This shows that within the experimental uncertainty the data are consistent with macropore diffusion control.

As a final test to confirm macropore diffusion we also changed the carrier gas. There was a clear difference between the two cases (not shown) and is once again further evidence of macropore diffusion in the pellets. Independent measurements carried out at VU confirm the results as well. Measurements have been carried out also on $\mathrm{Mg} / \mathrm{DOBDC}$ pellets and these also show macropore diffusion as the main mass transfer resistance. 


\subsection{Water and steam effects on $\mathrm{CO}_{2} \underline{\text { adsorption }}$}

\section{High-throughput hydrothermal stability: experiment and theory ${ }^{54}$}

Our objective was to understand relative stabilities of MOFs through experimental testing combined with Virtual High Throughput Screening (VHTS). This should enable the design of materials with improved hydrothermal stability, while maintaining the properties of interest. The tools that we have employed for these studies include quantum mechanical calculations based upon cluster models for VHTS and combinatorial steaming methods (see Figure 2 in Experimental section). A steam stability map (see Figure 27) was formulated according to the relative stability of different MOF materials. The experimental steaming method allows for high throughput screening of materials stability over a range of steam levels as well as in-depth investigation of structural transformations, while the cluster model presented here yields the correct trends in hydrothermal stability. For example, the inset in Figure 27 shows the interaction of a molecule of water with HKUST-1 to ultimately yield a broken metal linker bond and displaced linker.

Good agreement was observed between predicted relative stabilities of materials by VHTS and experimental results. Fundamental information from these studies has provided insight into how metal composition and coordination, and chemical functionality of organic linker affect the relative stabilities of MOFs. This work suggests that the strength of the bond between the metal oxide cluster and the bridging linker is important in determining the hydrothermal stability of the MOF.

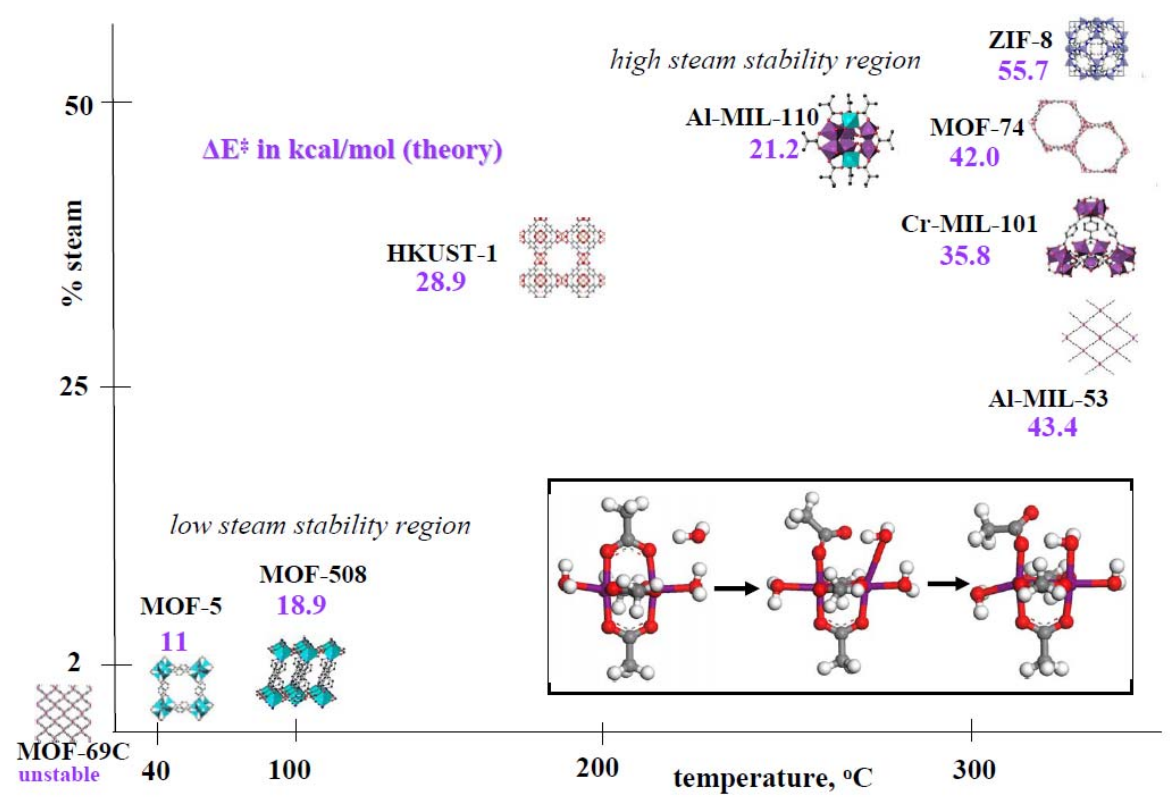

Figure 27. A steam stability map showing relative hydrothermal stability for several common MOFs. Values in purple are calculated, while placement of the MOF on the graph is from experimental results. The inset shows a molecule of water displacing part of a dicarboxylate linker-metal bond. 
A summary of the calculated and experimentally determined hydrothermal stabilities is shown in Table 7. The correlation between theory and experiment is quite good.

Table 7. Calculated and experimentally determined hydrothermal stabilities for several key MOFs.

\begin{tabular}{|c|c|c|c|}
\hline MOF & SBU & $\Delta \mathrm{E} \neq$ disp (theory) & $\begin{array}{l}\text { experimental maximum } \\
\text { stability }\end{array}$ \\
\hline Zn-MOF-69C & chain & unstable & $0 \%$ steam, ambient \\
\hline Co-CUK-1 & $\mathrm{Co}_{3}\left(\mu_{3}-\mathrm{OH}\right)_{2}{ }^{2+}$ & 8.2 & $50 \%$ steam, $200{ }^{\circ} \mathrm{C}$ \\
\hline Zn-MOF-5 & $\mathrm{Zn}_{4} \mathrm{O}^{6+}$ & 11.6 & $2 \%$ steam, $40^{\circ} \mathrm{C}$ \\
\hline $\mathrm{Zr}-\mathrm{UiO}-66$ & $\mathrm{Zr}_{6} \mathrm{O}_{4}(\mathrm{OH})_{4}{ }^{6+}$ & 14.0 & low thermal stability \\
\hline La-PDA & triple helix & 17.0 & $30 \%$ steam, $150{ }^{\circ} \mathrm{C}$ \\
\hline Zn-MOF-508 & $\mathrm{Zn}_{2}{ }^{2+}$ & 18.9 & $5 \%$ steam, $100^{\circ} \mathrm{C}$ \\
\hline Tb-MOF-76 & chain & 20.9 & $5 \%$ steam, $175^{\circ} \mathrm{C}$ \\
\hline Cu-HKUST-1 & $\mathrm{Cu}_{2}{ }^{2+}$ & 28.9 & $50 \%$ steam, $200{ }^{\circ} \mathrm{C}$ \\
\hline V-MIL-47 & chain & 30.9 & to be determined \\
\hline Cr-MIL-101 & $\mathrm{Cr}_{3} \mathrm{OF}^{6+}$ & 35.8 & $50 \%$ steam, $325^{\circ} \mathrm{C}$ \\
\hline $\mathrm{Co} / \mathrm{DOBDC}$ & chain & 35.8 & $50 \%$ steam, $250{ }^{\circ} \mathrm{C}$ \\
\hline $\mathrm{Mg} / \mathrm{DOBDC}$ & chain & 40.2 & $50 \%$ steam, $275^{\circ} \mathrm{C}$ \\
\hline Zn-MOF-74 & chain & 42.0 & $50 \%$ steam, $300{ }^{\circ} \mathrm{C}$ \\
\hline Al-MIL-53 & chain & 43.4 & $25 \%$ steam, $325{ }^{\circ} \mathrm{C}$ \\
\hline Ga-MIL-68 & chain & 43.7 & $50 \%$ steam, $325^{\circ} \mathrm{C}$ \\
\hline Al-MIL-110 & $\mathrm{Al}_{8}(\mathrm{OH})_{15}{ }^{9+}$ & 55.9 & $40 \%$ steam, $350{ }^{\circ} \mathrm{C}$ \\
\hline Zn-ZIF-8 & $\mathrm{Zn}^{2+}$ & 58.5 & $50 \%$ steam, $350{ }^{\circ} \mathrm{C}+$ \\
\hline
\end{tabular}

Modeling the influence of Water. The team also investigated the effect of coordinated water molecules that can be present in some MOFs. These water molecules are coordinated with the open metal sites and unlike the free water molecules present in the pores they are not fully mobile. It is a common practice to activate MOFs by heating them to high temperatures to ensure solvent removal. Any water present can also be removed. However, it requires higher temperatures to remove the coordinated water molecules than the loosely-bound water. We chose HKUST-1 as a test case. In HKUST-1 there is one coordinated water molecule per $\mathrm{Cu}$ atom. The original crystallographic data contains the position of the oxygen atoms of the coordinated water molecules but not the hydrogen atoms. We used DFT calculations to place the hydrogen atoms, as shown in Figure 28. 
GCMC simulations were performed to calculate $\mathrm{CO}_{2}$ adsorption isotherms for the completely dehydrated MOF and the hydrated MOF (4 wt \%) at $298 \mathrm{~K}$. The predicted isotherms are shown in Figure 29 in red. ${ }^{55}$ Over the pressure range considered, the presence of coordinated water molecules increases the adsorption of $\mathrm{CO}_{2}$. At the lowest pressures, the increase is substantial. For example, at 0.1 bar, the hydrated HKUST-1

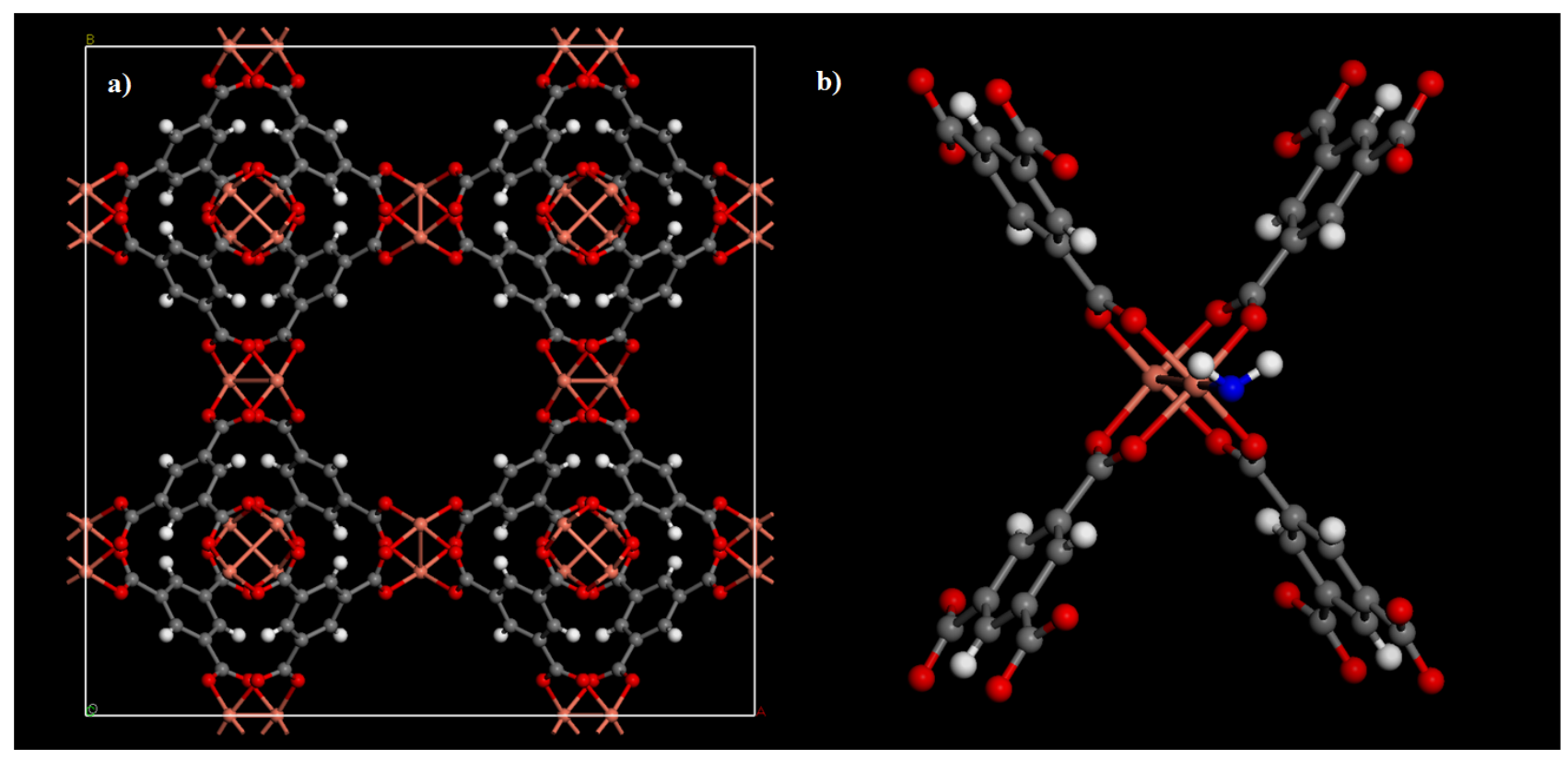

Figure 28. a) Dry HKUST-1 unit cell, b) hydrated HKUST-1 (4 wt $\%$ ) with coordinated water molecule from DFT. Copper atoms are orange, oxygens red, carbons gray, and hydrogens white. The oxygen atom of the coordinated water molecule is shown in blue.

adsorbs $71 \%$ more $\mathrm{CO}_{2}$ than the dry HKUST-1. At 1 bar, the increase is around $45 \%$, and at 2 bar $32 \%$. To test our prediction, we performed experimental adsorption measurements of $\mathrm{CO}_{2}$ in HKUST-1 with different levels of hydration. The experimental isotherms are shown in Figure 29 in black along with the simulated results. Over the entire pressure range examined, the experimental sample loaded with $4 \mathrm{wt} \%$ water adsorbs more $\mathrm{CO}_{2}$ than the dry sample, confirming the trend predicted by simulation.

A detailed analysis of simulation data revealed that interaction between the quadrupole moment of $\mathrm{CO}_{2}$ and the electric field created by the water molecules is responsible for the enhanced $\mathrm{CO}_{2}$ uptake. These findings open new possibilities for tuning the adsorption behavior of MOFs for $\mathrm{CO}_{2}$ capture and other applications. They also indicate that in some cases, the presence of some water is beneficial rather than detrimental to $\mathrm{CO}_{2}$ uptake. 


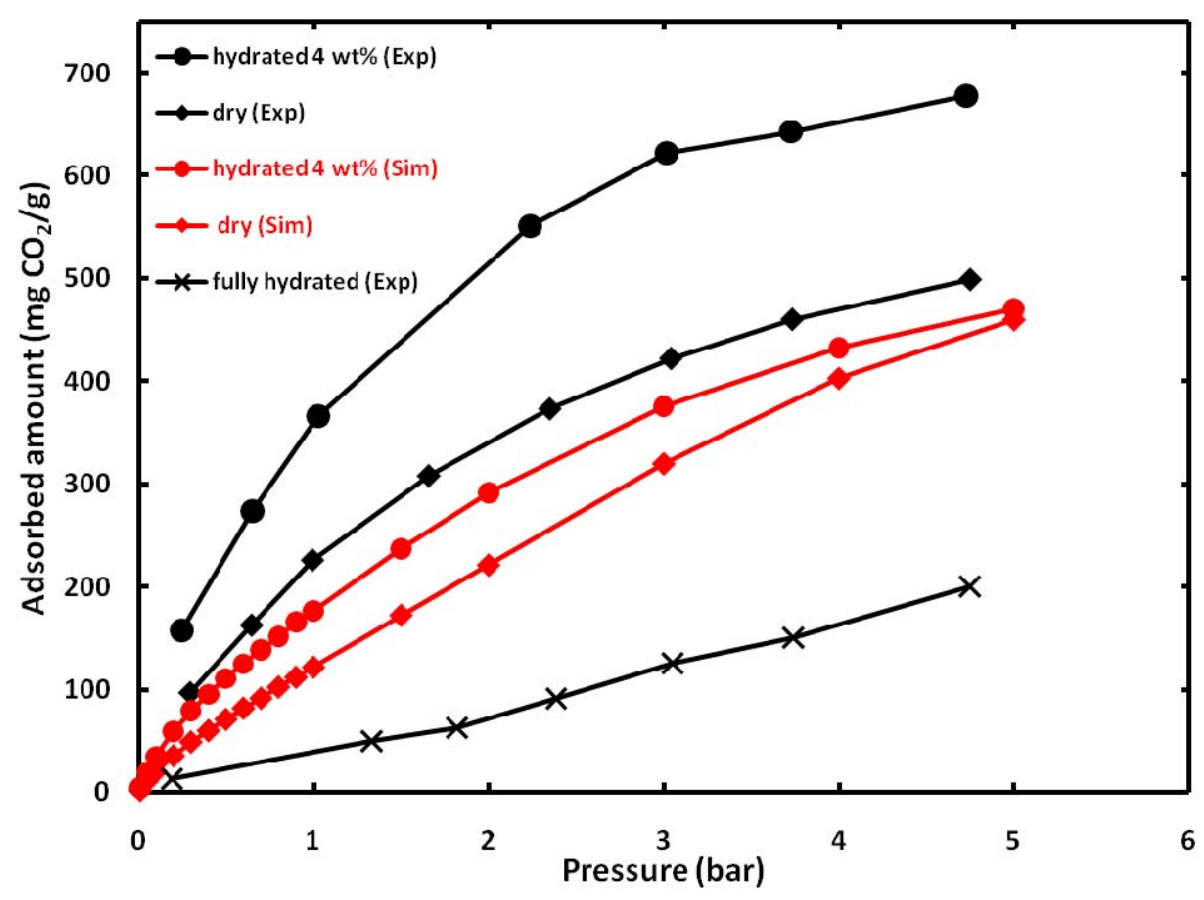

Figure 29. Comparison of simulated and experimental adsorption isotherms for $\mathrm{CO}_{2}$ at $298 \mathrm{~K}$ in HKUST-1 with different water contents.

\section{$\underline{\mathrm{CO}_{2}} \underline{\text { capacity and water stability performance milestones }}$}

The team's end of Phase $2 \mathrm{CO}_{2}$ capacity performance target was $15 \mathrm{wt} \% \mathrm{CO}_{2}$ capacity at $20{ }^{\circ} \mathrm{C}$ at up to 2 atmospheres pressure. Many MOFs were easily able to achieve this target (see Table 8). In fact, as can been seen in Table 8, it is expected that most of these MOFs can achieve the end of Phase 3 capacity target of $15 \mathrm{wt} \% \mathrm{CO}_{2}$ capacity at $38{ }^{\circ} \mathrm{C}$ at one atmosphere of $\mathrm{CO}_{2}$ pressure. Because flue gas has only about 0.1 atmosphere $\mathrm{CO}_{2}$ pressure at $38{ }^{\circ} \mathrm{C}$, a column indicating adsorption capacity under these conditions is also provided in Table 8.

Table 8. MOFs exceeding end Phase 2 (and end Phase 3) $\mathrm{CO}_{2}$ capacity performance.

\begin{tabular}{|c|c|c|c|}
\hline Sample & $\begin{array}{c}\text { Loading at } 0.1 \mathrm{~atm} \text { and } \\
38 \mathrm{deg} \mathrm{C}(\mathrm{wt} \%)\end{array}$ & $\begin{array}{c}\text { Loading at } 1 \mathrm{~atm} \text { and } \\
25 \mathrm{degC}(\mathrm{wt} \%) \\
\end{array}$ & $\begin{array}{c}\text { Projected loading at } 2 \text { atm } \\
\text { and } 25 \operatorname{degC}\left(\mathrm{wt}^{\circ} \%\right)\end{array}$ \\
\hline $\mathrm{Mg} \backslash \mathrm{DOBDC}$ & 20.9 & 37.1 & 46 \\
\hline HKUST-1 ( $4 \%$ water) & na & 37.0 & $53^{*}$ \\
\hline NilDOBDC & 15.0 & 30.9 & 38 \\
\hline Co\DOBDC & 8.1 & 29.2 & 37 \\
\hline HKUST-1 (dry) & $1.8^{* *}$ & 22.0 & $35^{*}$ \\
\hline $\mathrm{Zn} \backslash \mathrm{DOBDC}$ & 1.8 & 15.9 & 22 \\
\hline
\end{tabular}

*actually determined values **lower quality sample 
The end of Phase 2 minimum stability performance target was retention of $75 \% \mathrm{CO}_{2}$ capacity after exposure of material to $3-7$ mole $\%$ steam at $100{ }^{\circ} \mathrm{C}$ for 2 hours. The end of Phase 2 targets were achieved with the team's Number 2 MOF, Ni/DOBDC, as shown in Table 9.

Table 9. End Phase 2 stability performance: $\mathrm{CO}_{2}$ adsorption on $\mathrm{Ni} / \mathrm{DOBDC}$ formed into pellets and alumina-bound extrudates and then steamed at $100{ }^{\circ} \mathrm{C}$ in 5 mole $\%$ steam for $2 \mathrm{~h}$.

\begin{tabular}{|l|c|c|c|}
\hline sample & CO2 capacity (mol/kg) & $\begin{array}{c}\text { CO2 capacity } \\
\text { (wt\%) }\end{array}$ & $\begin{array}{c}\text { ave. heat of adsorption } \\
\text { (kJ/mol) }\end{array}$ \\
\hline unsteamed powders (several samples) & $2.98-3.09$ & $13.2-13.7$ & 38 to 41 \\
\hline extrudate with 2\% alumina & 2.96 & 13.1 & 43 \\
\hline steamed* powder & 2.73 & 12.1 & 33 \\
\hline unsteamed pellet & 2.68 & 11.8 & 35 \\
\hline steamed* pellet & 2.23 & 9.9 & 36 \\
\hline & total CO2 capactity loss: $\mathbf{2 5 - 2 7 \%}$ & \\
\hline
\end{tabular}

* conditions: $100 \mathrm{deg} \mathrm{C}, 5 \mathrm{~mol} \%$ steam for $2 \mathrm{~h}$

The performance of the $\mathrm{Mg} / \mathrm{DOBDC}$ samples was even more significantly impacted by the moderate severity end of Phase 2 steaming conditions employed here. See Table 10.

Table 10. End Phase 2 stability performance: $\mathrm{CO}_{2}$ adsorption on $\mathrm{Mg} / \mathrm{DOBDC}$ formed into pellets and then steamed at $100{ }^{\circ} \mathrm{C}$ in 5 mole $\%$ steam for $2 \mathrm{~h}$.

\begin{tabular}{|l|c|c|c|}
\hline sample & $\begin{array}{c}\text { CO2 capacity } \\
(\mathbf{m o l} / \mathbf{k g})\end{array}$ & $\begin{array}{c}\text { CO2 capacity } \\
(\mathbf{w t} \%)\end{array}$ & $\begin{array}{c}\text { CO2 capacity loss (\%) relative to } \\
\text { unsteamed Mg/DOBDC powder }\end{array}$ \\
\hline unsteamed Mg/DOBDC 2010 powder & 4.93 & 21.7 & 0.0 \\
\hline unsteamed pressed pellet & 4.86 & 21.4 & 1.4 \\
\hline steamed* powder & 2.41 & 10.6 & 51.1 \\
\hline steamed* pellet & 1.9 & 8.4 & $60.9 * *$ \\
\hline
\end{tabular}

* conditions: $100 \mathrm{deg} \mathrm{C}, 5 \mathrm{~mol} \%$ steam for $2 \mathrm{~h}$

** relative to unsteamed pellet

The team's focus in Phase 3 was upon the M/DOBDC series $(\mathrm{M}=\mathrm{Mg}, \mathrm{Zn}, \mathrm{Co}$, or Ni) and HKUST-1 owing to high $\mathrm{CO}_{2}$ capacity and good steam stability (Tables 8 and 9). An optimized $\mathrm{Mg} / \mathrm{DOBDC}$ sample provided by UM outperformed all MOF and zeolite materials evaluated to date, as measured at VU and UE. $\mathrm{CO}_{2}$ adsorption modeling results from NU suggest that modified versions of $\mathrm{Mg} / \mathrm{DOBDC}$ could perform even better. Therefore, the most critical materials-related activity in Phase 3 was optimization, scale- 
up and forming of $\mathrm{Mg} / \mathrm{DOBDC}$ materials. Table 2 summarizes results for the overall best performers at the end of the Project.

The overall objective of Phase 3 was to demonstrate one or more MOF materials that meet performance targets and have sufficient stability to carry into larger scale testing. End of Phase 3 performance targets are $15 \mathrm{wt} \% \mathrm{CO}_{2}$ capacity at $38-40{ }^{\circ} \mathrm{C}$ at up to 1.25 atmospheres pressure. As shown in Table 7 above, this has been achieved by several MOF materials, including several members of the M/DOBDC family, as well as HKUST1. A minimum stability target was retention of $75 \% \mathrm{CO}_{2}$ capacity after exposure of material to up to 15 mole $\%$ steam at $150{ }^{\circ} \mathrm{C}$ for up to 24 hours. But based upon the end Year 2 results reported in Table 8, it was obvious that we would not be able to meet this target with the same MOF material (Ni/DOBDC). Nevertheless, for the sake of completeness we carried out the steaming experiments on Ni/DOBDC samples and report the results in Table 11. As expected, the performance of the Ni/DOBDC samples was significantly impacted by the high severity steaming conditions. Even though the steaming conditions employed here are much harsher than would be expected in the field, these results nevertheless strengthen the team's expectation that Ni/DOBDC or any other high $\mathrm{CO}_{2}$ capacity and selective MOF would need to be protected by a drying bed before $\mathrm{CO}_{2}$ capture.

In summary, while the very stringent stability goal was not achieved, the Project was able to meet its material performance capacity objectives with its Number 2 (Ni/DOBDC) material and even with its Number 3 (HKUST-1) material. It should be noted that the stability goal in no way suggests that MOFs cannot be used in this application. Instead, this result suggests that the MOF adsorbent would need to be protected by a guard (drying) bed in commercial operation.

Table 11. The combined effects of forming Ni/DOBDC into pellets and alumina-bound extrudates and then severely steaming the pellets on $\mathrm{CO}_{2}$ adsorption.

\begin{tabular}{|l|c|c|c|}
\hline sample & $\begin{array}{c}\text { CO2 capacity } \\
(\mathbf{m o l} / \mathbf{k g})\end{array}$ & $\begin{array}{c}\text { CO2 capacity } \\
(\mathbf{w t} \%)\end{array}$ & $\begin{array}{c}\text { CO2 capacity loss (\%) relative to } \\
\text { unsteamed Ni/DOBDC powder }\end{array}$ \\
\hline unsteamed Ni/DOBDC 2010 powder & 1.66 & 7.3 & 0 \\
\hline extrudate with 2\% alumina & 1.31 & 5.8 & 21 \\
\hline unsteamed pressed pellet & 1.06 & 4.7 & 36 \\
\hline steamed* extrudate & 0.6 & 2.6 & $55^{* *}$ \\
\hline steamed* powder & 0.47 & 2.1 & 71 \\
\hline steamed* pellet & 0.39 & 1.7 & $64 * * *$ \\
\hline
\end{tabular}

* conditions: $150 \mathrm{deg} \mathrm{C}, 15 \mathrm{~mol} \%$ steam for $4 \mathrm{~h}$

** relative to unsteamed extrudate

*** relative to unsteamed pellet 


\subsection{Fundamentals: effects of water, simulated flue gas, and cycling on key MOFs}

Several detailed fundamental studies were carried out in Phase 3 of the project to address $\mathrm{CO}_{2}$ adsorption on MOFs that have been exposed to water and other contaminants. Some work with zeolites was also carried out for reference point. A difficulty in using traditional zeolites $\left(5 \mathrm{~A}, \mathrm{NaX}\right.$, etc.) to capture $\mathrm{CO}_{2}$ from flue gas is the adsorption of $\mathrm{H}_{2} \mathrm{O}$, which is contained in the flue gases and is strongly adsorbed on the zeolites. ${ }^{56}$ In addition, the presence of a trace amount of water can significantly affect the $\mathrm{CO}_{2}$ capacities of the zeolites. ${ }^{57}$ Therefore, it is important to study the $\mathrm{H}_{2} \mathrm{O}$ effect during the investigation of $\mathrm{CO}_{2}$ adsorption in other microporous materials like MOFs.

In a process design using a MOF or another adsorbent, it may be advantageous to utilize a guard bed to adsorb water and thereby minimize $\mathrm{H}_{2} \mathrm{O}$ effects on the adsorbent targeted for $\mathrm{CO}_{2}$ capture. Most MOF materials are considered to have hydrophilic surfaces, which generally have strong interactions with $\mathrm{H}_{2} \mathrm{O}$ molecules. ${ }^{58}$ However, the $\mathrm{H}_{2} \mathrm{O}$ adsorption process in some MOF materials, such as HKUST-1, is reversible, and the adsorbed $\mathrm{H}_{2} \mathrm{O}$ can be thoroughly removed under moderate temperatures. ${ }^{59}$ Experimental and simulation room temperature $\mathrm{H}_{2} \mathrm{O}$ isotherm data have been reported for HKUST- $1 .{ }^{60}$ Results have revealed some characteristics for $\mathrm{H}_{2} \mathrm{O}$ adsorption in HKUST-1 but have not provided information on the effect of $\mathrm{H}_{2} \mathrm{O}$ on adsorption of $\mathrm{CO}_{2}$ or other gases. Team members ${ }^{55}$ have recently reported interesting simulation and experimental results on enhancing the $\mathrm{CO}_{2}$ capacities for HKUST-1 by pre-adsorbing $4 \mathrm{wt} \% \mathrm{H}_{2} \mathrm{O}$. More research is needed to address higher loadings of water, such as those that would be encountered in capturing $\mathrm{CO}_{2}$ from wet flue gas. Especially, extensive experimental studies are still needed to help better understand the $\mathrm{H}_{2} \mathrm{O}$ effect on $\mathrm{CO}_{2}$ capture from flue gas by using MOF materials as adsorbents. Besides $\mathrm{CO}_{2}$ and $\mathrm{H}_{2} \mathrm{O}$, typical flue gases also contains trace amounts of acid gases such as $\mathrm{NO}_{\mathrm{x}}$ and $\mathrm{SO}_{\mathrm{x}}{ }^{61}$ Therefore, it is also necessary to investigate the impact of exposure to acid gas and moisture on $\mathrm{CO}_{2}$ adsorption in MOF samples.

\section{$\underline{\text { General observations and experimental adjustments }}$}

ZLC. It became apparent to team members at UE that the ZLC was particularly sensitive to water. Compared to the gravimetric experiments, the ZLC offers some advantage in terms of the time required for the measurements, but where it becomes very useful is in the determination of the effect of water. With the dosing system that was developed for this project, team members were able to carry out experiments without any water and then expose the sample to water and $\mathrm{CO}_{2}$ and measure the resulting $\mathrm{CO}_{2}$ capacity.

As an example consider the results for $\mathrm{Ni} / \mathrm{DOBDC}$, which is stable in the presence of water, but is also strongly hydrophilic. For this experiment, one of the issues that needed probing was the amount of time needed to saturate fully the sample. Initial tests had been run exposing the column to water and $\mathrm{CO}_{2}$ for only two hours, but as a result of the experiments on initial activation, shown in Figure 30, it became apparent that the sample could adsorb up to $33 \%$ by weight of water and at the concentration and flow rates being 
used, this would require several hours. Figure 30 shows the blank ZLC runs. The transient for $\mathrm{CO}_{2}$ is very fast, while water takes approximately 2 to 3 hours to reach saturation. This difference indicates that at least in part water adsorbs on the metal surfaces of the mass spectrometer or in residual adsorbent particles in the lines.

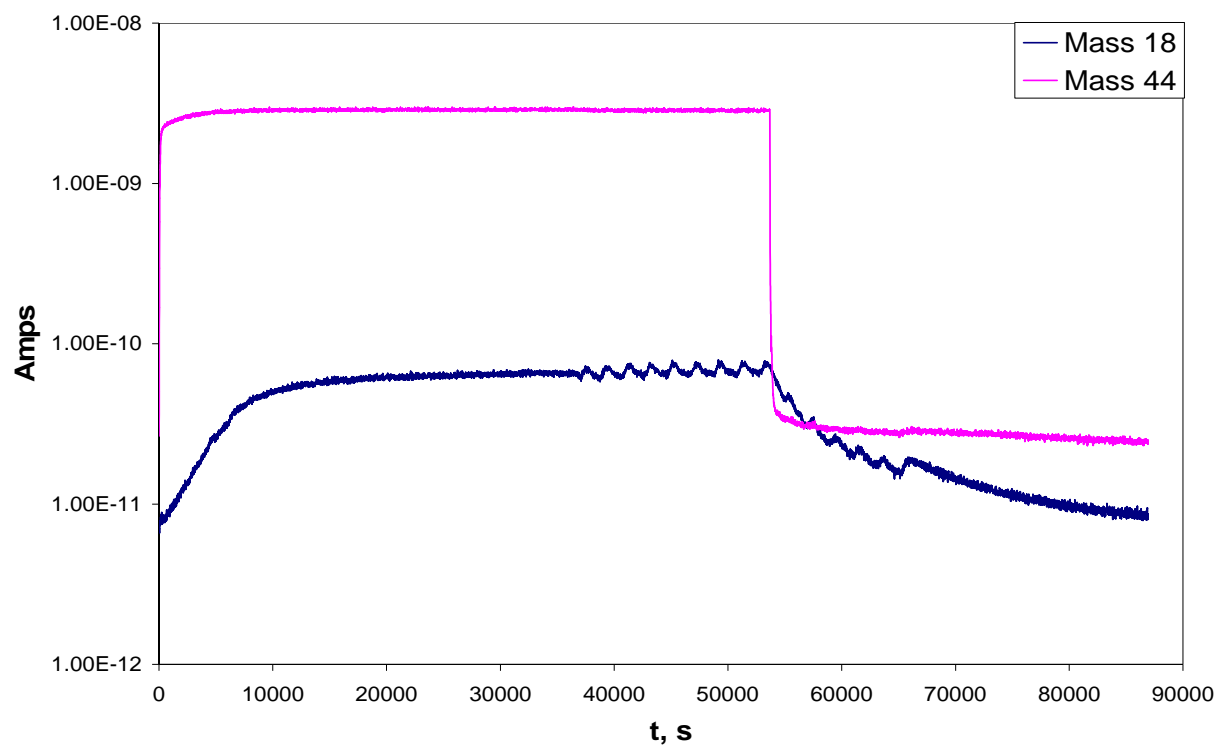

Figure 30. Blank ZLC runs with water and $\mathrm{CO}_{2}$.

Figure 31 shows the result for the ZLC column with Ni/DOBDC.

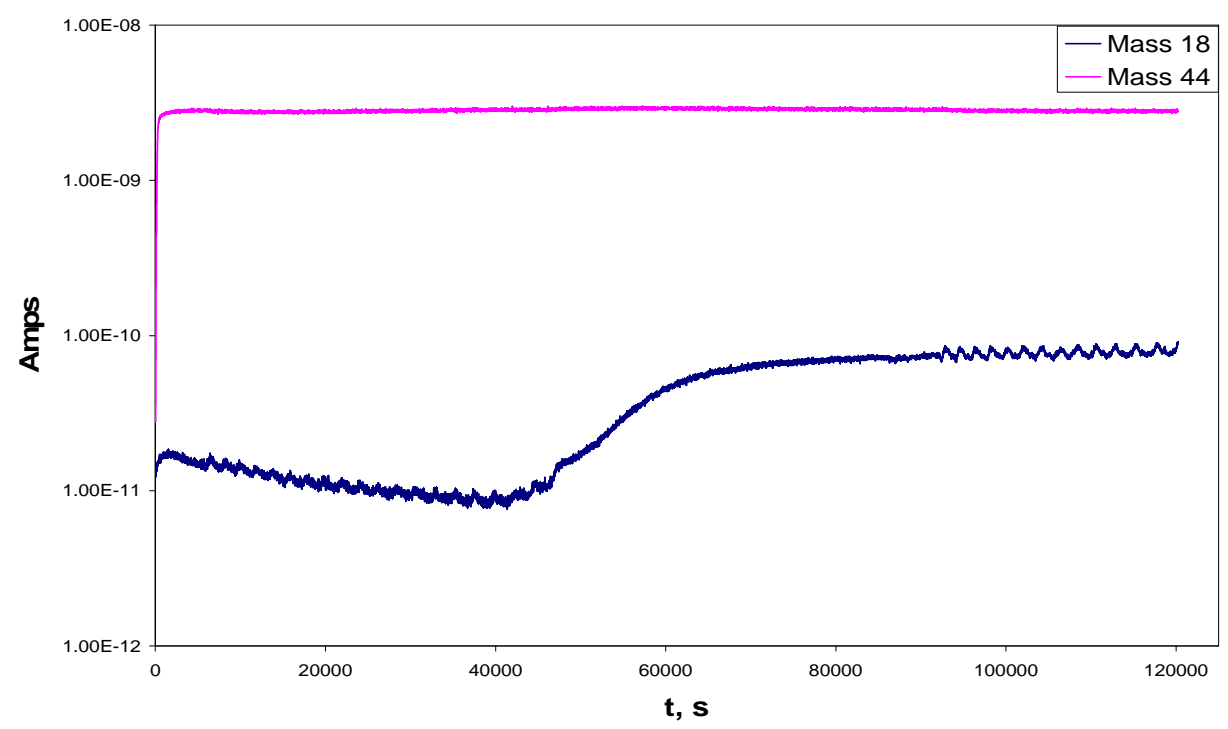

Figure 31. $\mathrm{ZLC}$ runs with water and $\mathrm{CO}_{2}$.

In this case saturation is achieved only after more than 6 hours. Figure 32 shows the comparison of the curves with the dry sample runs and the results from the experiments with only partial water equilibration ( 2 hours). These experiments indicate that this adsorbent, once saturated with water does not have an appreciable capacity for $\mathrm{CO}_{2}$. This 
means that in a process this adsorbent could be used only in a layered bed, with an initial layer that adsorbs the water, or with a gas drying unit before the $\mathrm{CO}_{2}$ capture section.

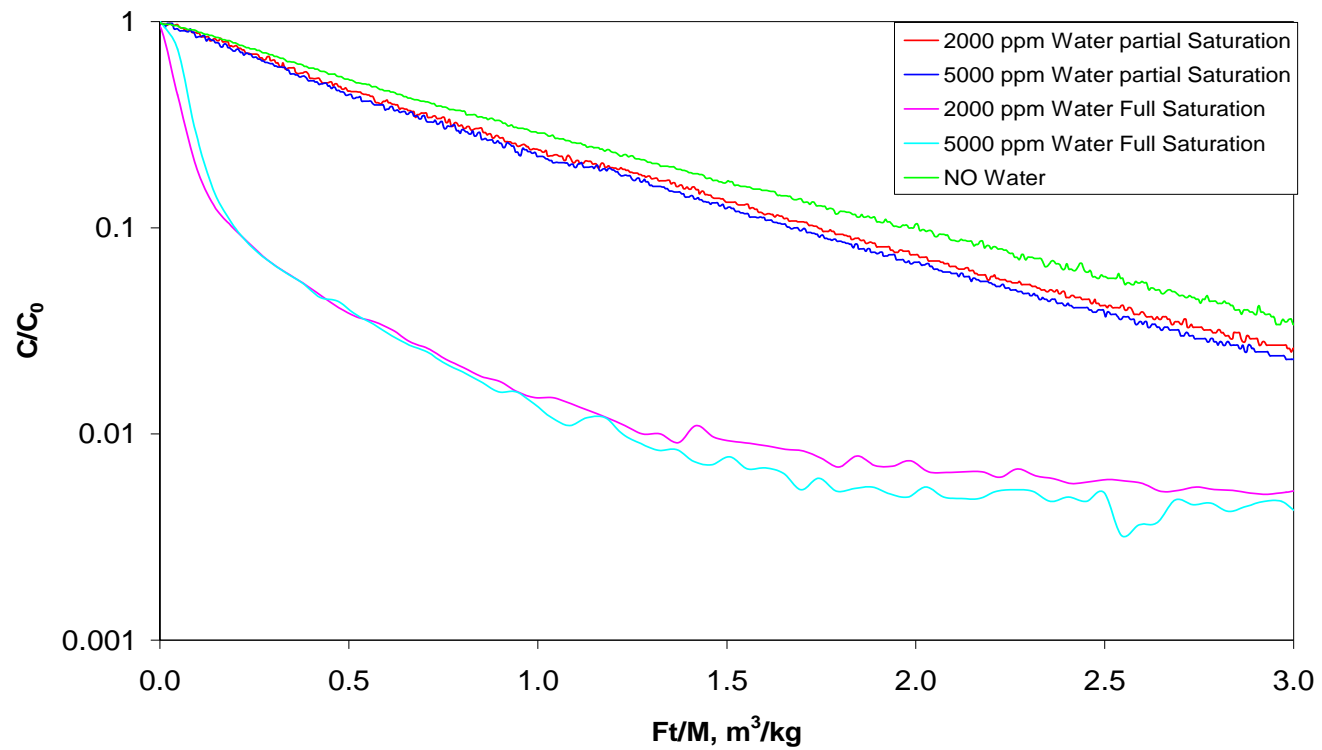

Figure 32. Comparison of $\mathrm{CO}_{2}$ capacities on $\mathrm{Ni} / \mathrm{DOBDC}$ in the presence of water.

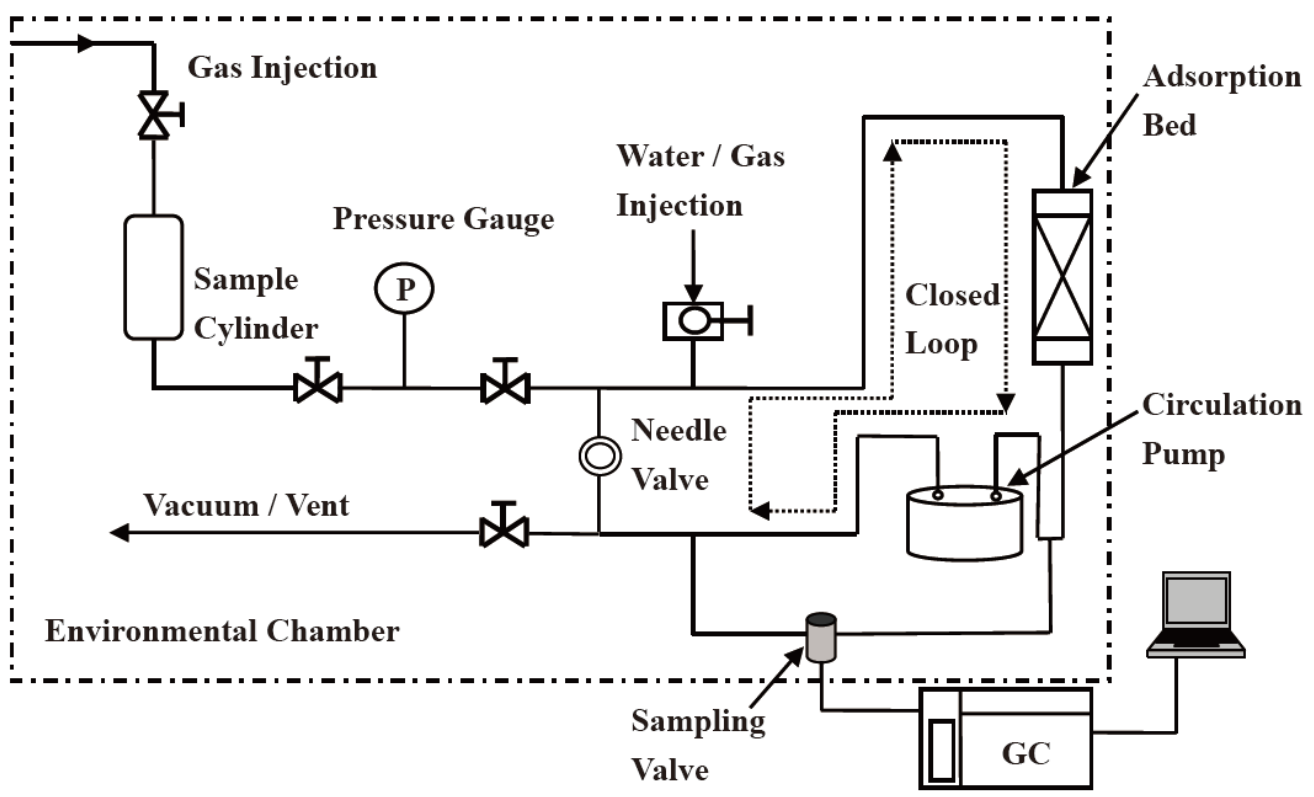

Figure 33. Volumetric system for $\mathrm{CO}_{2} / \mathrm{H}_{2} \mathrm{O}$ adsorption equilibrium measurement. 
Volumetric measurements. Figure 33 shows a schematic diagram of the volumetric system used in our experiments to measure the $\mathrm{CO}_{2}$ and $\mathrm{H}_{2} \mathrm{O}$ adsorption equilibrium. Pellet MOF samples were pressed from pure powder samples without any binder. Note that pelletizing without binder can cause about $5 \%$ decrease in the $\mathrm{CO}_{2}$ capacity for MOF samples based on our experience. Before measurements, HKUST-1 samples were regenerated at $170{ }^{\circ} \mathrm{C}$ for $8 \mathrm{~h}$ under high vacuum $\left(1 \times 10^{-5} \mathrm{~Pa}\right)$ to obtain the fresh sample weight and regenerated again in situ at $170{ }^{\circ} \mathrm{C}$ for $12 \mathrm{~h}$ under vacuum with helium flow. Similarly, Ni/DOBDC samples were regenerated at $150{ }^{\circ} \mathrm{C}$ for $8 \mathrm{~h}$ under high vacuum $(1$ $\times 10^{-5} \mathrm{~Pa}$ ) to obtain the fresh sample weight and regenerated again in situ at $150{ }^{\circ} \mathrm{C}$ for 12 $\mathrm{h}$ under vacuum with helium flow. $\mathrm{Mg} / \mathrm{DOBDC}$ samples were regenerated at $200{ }^{\circ} \mathrm{C}$ for $8 \mathrm{~h}$ under high vacuum $\left(1 \times 10^{-5} \mathrm{~Pa}\right)$ to obtain the fresh sample weight and regenerated again in situ at $200{ }^{\circ} \mathrm{C}$ for $12 \mathrm{~h}$ under vacuum with helium flow.

After regeneration, gases and water vapor were introduced into the system by flow and liquid injection, respectively. These were circulated in the closed loop by the circulation pump at a rate of about $1.0 \mathrm{~L} / \mathrm{min}$. The whole apparatus was contained inside an environmental chamber (Thermotron SE-300) to keep temperature constant. Equilibrium was determined by using a gas chromatograph (GC, HP-6890) with a thermal conductivity detector (TCD). In the $\mathrm{CO}_{2} / \mathrm{H}_{2} \mathrm{O}$ binary equilibrium experiments, water was first injected into the system. After reaching equilibrium, the water loadings were constant (varied $1.2 \%$ of their values) throughout the $\mathrm{CO}_{2}$ isotherm measurement processes.

\section{$\underline{\text { Pure } \mathrm{CO}_{2}} \underline{\text { and } \mathrm{H}_{2}} \underline{\mathrm{O} \text { isotherms }}$}

Pure component isotherms form the basis for understanding mixture adsorption. Figure

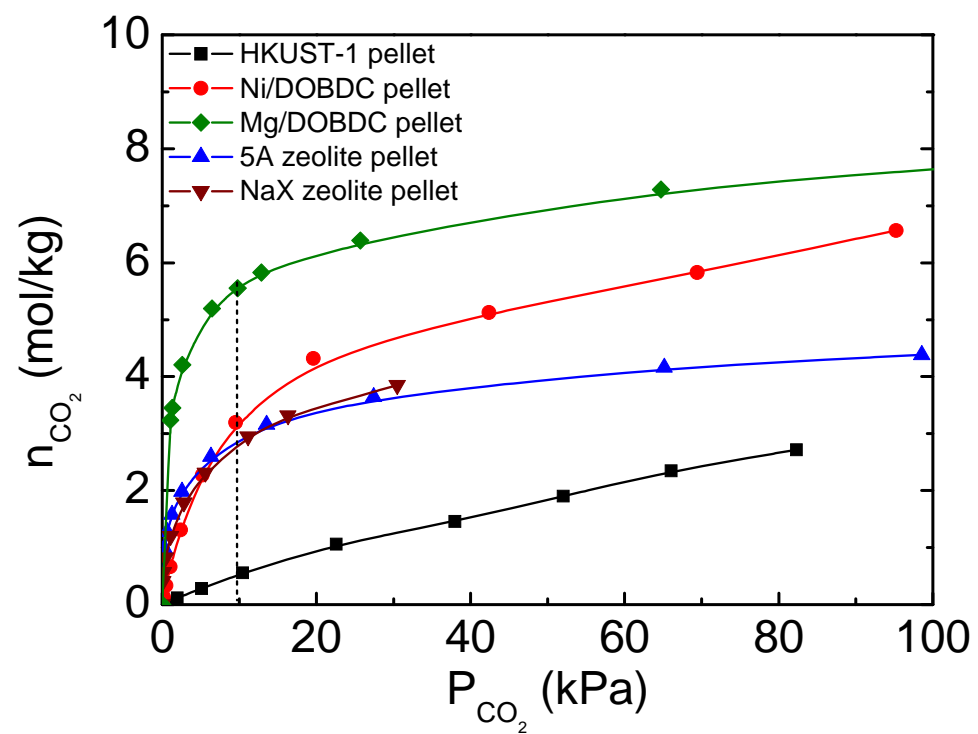

Figure 34. $\mathrm{CO}_{2}$ isotherms at $25{ }^{\circ} \mathrm{C}$ for MOF and zeolite pellets. Curves are multi temperature Toth equation fits. Zeolite results are taken from the literature. $^{[23]}$ 
34 shows pure $\mathrm{CO}_{2}$ isotherms at $25^{\circ} \mathrm{C}$ for HKUST-1, Ni/DOBDC, and $\mathrm{Mg} / \mathrm{DOBDC}$ pellets. We also include the results ${ }^{62}$ for $5 \mathrm{~A}$ and $\mathrm{NaX}$ zeolite pellets (W. R. Grace) for comparison. From Figure 34, it is clear that the $\mathrm{CO}_{2}$ capacities for HKUST-1, $\mathrm{Ni} / \mathrm{DOBDC}$ and $\mathrm{Mg} / \mathrm{DOBDC}$ pellets at $0.1 \mathrm{~atm}$ are $0.55,3.28$, and $5.65 \mathrm{~mol} / \mathrm{kg}$, respectively. Given 48 metal atoms per unit cell for HKUST-1 and 18 for Ni/DOBDC and $\mathrm{Mg} / \mathrm{DOBDC}$, these uptake values correspond to $0.11 \mathrm{CO}_{2}$ molecules/metal atom (5.3 $\mathrm{CO}_{2}$ molecules/unit cell), $0.63 \mathrm{CO}_{2}$ molecules/metal atom $\left(11.3 \mathrm{CO}_{2}\right.$ molecules/unit cell), and $0.69 \mathrm{CO}_{2}$ molecules/metal atom (12.4 $\mathrm{CO}_{2}$ molecules/unit cell) for HKUST-1, $\mathrm{Ni} / \mathrm{DOBDC}$, and $\mathrm{Mg} / \mathrm{DOBDC}$, respectively. This indicates that the adsorption sites in $\mathrm{Ni} / \mathrm{DOBDC}$ and $\mathrm{Mg} / \mathrm{DOBDC}$ are more attractive to $\mathrm{CO}_{2}$ than those in HKUST-1.

It is interesting to note that $\mathrm{Ni} / \mathrm{DOBDC}$ and $\mathrm{Mg} / \mathrm{DOBDC}$ have even higher $\mathrm{CO}_{2}$ capacities than $5 \mathrm{~A}$ and $\mathrm{NaX}$ zeolites at $0.1 \mathrm{~atm}$. The high gas capacities at low pressures have been ascribed to the open metal sites a.k.a. unsaturated metal centers (UMCs) in the crystal structures of these MOFs. ${ }^{63}$ The $\mathrm{Cu}$ atoms in HKUST-1, Ni atoms in Ni/DOBDC, and $\mathrm{Mg}$ atoms in $\mathrm{Mg} / \mathrm{DOBDC}$ are unsaturated after complete dehydration. These UMCs can coordinate with $\mathrm{CO}_{2}$ molecules and form strong adsorption interactions, which lead to high $\mathrm{CO}_{2}$ capacities for the MOF samples. ${ }^{64}$ However, the coordinate strength for the UMCs toward $\mathrm{CO}_{2}$ molecules varies from one MOF to another. A good example is the different $\mathrm{CO}_{2}$ capacities per metal atom for HKUST-1 and Ni/DOBDC. The difference can be attributed to the stronger ionic character of the metal-oxide bond in the $\mathrm{Ni} / \mathrm{DOBDC}$ as suggested in the literature. ${ }^{41 \mathrm{c}, 43}$

Pure $\mathrm{H}_{2} \mathrm{O}$ isotherms at $25^{\circ} \mathrm{C}$ for both the HKUST-1 and Ni/DOBDC pellets are shown in Figures 35 and 36. The $\mathrm{H}_{2} \mathrm{O}$ isotherm measurement was repeated several times, and we report the average results with standard deviations for the reproduced measurements shown as error bars. In Figure 35, some prior results for HKUST-1 are also included for comparison. Because of the differences in samples, our results and the two results from the literature do not match with each other very well. It has been reported that the same MOF synthesized using different procedures can have quite different adsorption characteristics. ${ }^{65}$ However, the high $\mathrm{H}_{2} \mathrm{O}$ capacities and steep slopes at low loadings shown in all three isotherms indicate strong $\mathrm{H}_{2} \mathrm{O}$ affinity for HKUST-1. Steps before reaching the saturation plateaus are also apparent in all of the isotherms. The saturated $\mathrm{H}_{2} \mathrm{O}$ capacity for HKUST-1 pellet is about $40 \mathrm{~mol} / \mathrm{kg}$.

The $25^{\circ} \mathrm{C} \mathrm{H}_{2} \mathrm{O}$ isotherm for Ni/DOBDC is shown in Figure 36. The $\mathrm{H}_{2} \mathrm{O}$ isotherm for $\mathrm{Ni} /$ DOBDC pellets is steeper than that for the HKUST-1 pellet, especially in the very low pressure region, which indicates stronger interactions between $\mathrm{H}_{2} \mathrm{O}$ molecules and the $\mathrm{Ni} / \mathrm{DOBDC}$ crystal structure. According to the literature, there is only one set of cylindrical pores, with a size of $11 \AA$, in the Ni/DOBDC framework while two sets of pores with sizes of 5 and $15 \AA$ exist in the HKUST-1 framework. ${ }^{66}$ In addition, the adsorption sites in the Ni/DOBDC framework are more attractive to $\mathrm{H}_{2} \mathrm{O}$ molecules. The $\mathrm{H}_{2} \mathrm{O}$ molecules can occupy adsorption sites in $\mathrm{Ni} / \mathrm{DOBDC}$ with uniformly fast rates, which leads to a shorter time to reach $80 \%$ of the saturation $\mathrm{H}_{2} \mathrm{O}$ capacity compared to 
HKUST-1 in which the two sets of pores with different sizes and less attractive adsorption sites to $\mathrm{H}_{2} \mathrm{O}$ molecules exist.

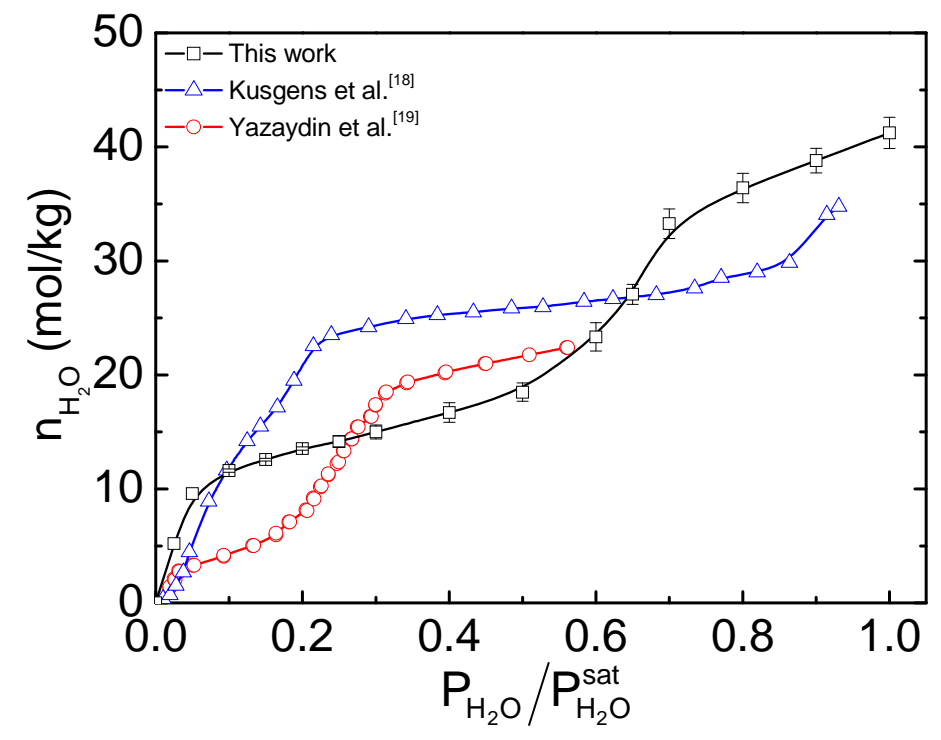

Figure 35. $\mathrm{H}_{2} \mathrm{O}$ isotherms at $25{ }^{\circ} \mathrm{C}$ for HKUST-1 pellet.

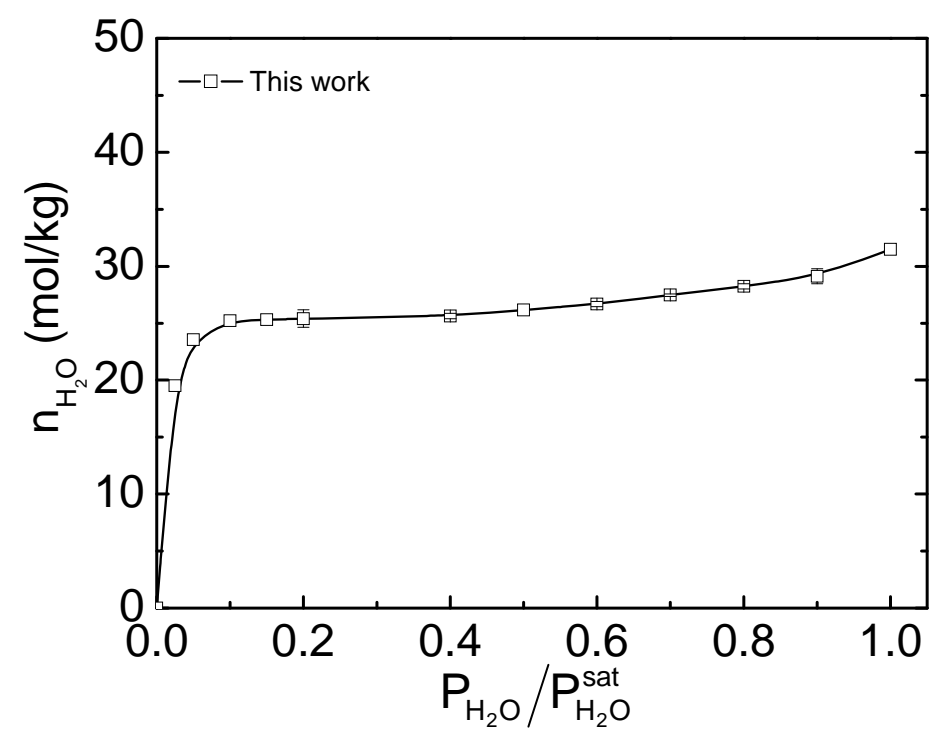

Figure 36. $\mathrm{H}_{2} \mathrm{O}$ isotherms at $25{ }^{\circ} \mathrm{C}$ for $\mathrm{Ni} / \mathrm{DOBDC}$ pellet.

The saturated $\mathrm{H}_{2} \mathrm{O}$ capacity for $\mathrm{Ni} / \mathrm{DOBDC}$ pellets is about $32 \mathrm{~mol} / \mathrm{kg}$. The saturated water capacity for $\mathrm{Ni} / \mathrm{DOBDC}$ is lower than that of HKUST-1, while the $\mathrm{CO}_{2}$ capacity for Ni/DOBDC shown in Figure 34 is higher than that for HKUST-1. For $\mathrm{CO}_{2}$ adsorption, our isotherms for both MOFs only show the relative pressure range from 0 to 0.016. In other words, the results in Figure 34 display the $\mathrm{CO}_{2}$ adsorption behavior under 
relatively low concentration at which stronger adsorption sites (i.e., UMCs) lead to higher $\mathrm{CO}_{2}$ capacities. The interaction between $\mathrm{UMCs}$ and $\mathrm{CO}_{2}$ molecules in $\mathrm{Ni} / \mathrm{DOBDC}$ is stronger than that in HKUST-1 as we know from previous results. In contrast, Figures 35 and 36 show the $\mathrm{H}_{2} \mathrm{O}$ adsorption behavior for the two MOFs up to high relative pressure at which larger surface area and pore volume usually lead to higher overall adsorption capacity. The HKUST-1 sample has a higher surface area and larger pore volume than the Ni/DOBDC sample. Consequently, it can adsorb more $\mathrm{H}_{2} \mathrm{O}$ than $\mathrm{Ni} / \mathrm{DOBDC}$ when the water vapor concentration is high.

\section{$\underline{\text { Water effects on } \mathrm{CO}_{2}}$ adsorption}

Some of these results were discussed in a more general way in section 3.4 above.

It is important to understand $\mathrm{H}_{2} \mathrm{O}$ effects on $\mathrm{CO}_{2}$ adsorption in adsorbent samples before making any conclusions about their applications in $\mathrm{CO}_{2}$ capture from wet flue gas. Figures 37,38 , and 39 show $\mathrm{CO}_{2}$ isotherms at $25^{\circ} \mathrm{C}$ for HKUST-1, Ni/DOBDC, and $\mathrm{Mg} /$ DOBDC pellets, respectively, with different $\mathrm{H}_{2} \mathrm{O}$ loadings. It is interesting to note from Figure 37 that a small amount of adsorbed water did not affect the $\mathrm{CO}_{2}$ capacity and may actually help to slightly increase $\mathrm{CO}_{2}$ adsorption in HKUST-1. As mentioned above, this been ascribed to the addition of coulombic interactions between $\mathrm{CO}_{2}$ molecules and $\mathrm{H}_{2} \mathrm{O}$ molecules after the introduction of $\mathrm{H}_{2} \mathrm{O}$ molecules into the system. The trend of our $\mathrm{CO}_{2}$ isotherms for HKUST-1 with low water loadings agrees quantitatively with this prediction. The $\mathrm{CO}_{2}$ capacity for HKUST-1 decreases when the $\mathrm{H}_{2} \mathrm{O}$ loading increases from 3.4 to $16.2 \mathrm{~mol} / \mathrm{kg}$. HKUST-1 has lost almost all of its $\mathrm{CO}_{2}$ capacity when the $\mathrm{H}_{2} \mathrm{O}$ loading is about $25.4 \mathrm{~mol} / \mathrm{kg}$, which is approaching the $\mathrm{H}_{2} \mathrm{O}$ saturation capacity ( $\mathrm{RH} \approx 67 \%$, where $\mathrm{RH}$ is the percent relative humidity, i.e., ${ }_{\mathrm{H}_{2} \mathrm{O}} / \mathrm{P}_{\mathrm{H}_{2} \mathrm{O}}^{\mathrm{sat}} \times 100 \%$ ).

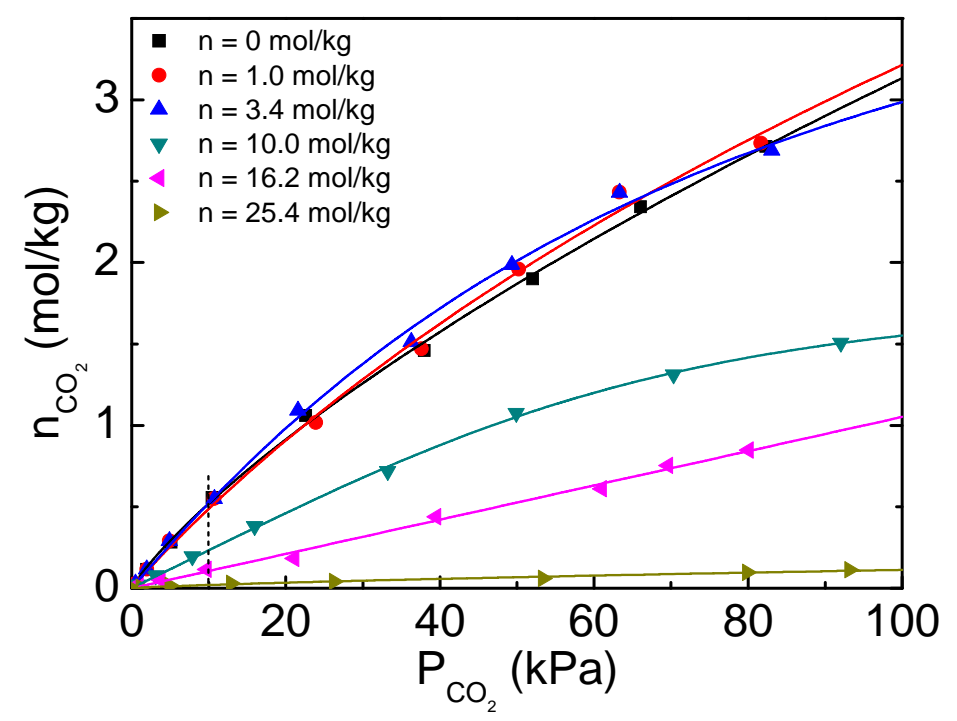

Figure 37. $\mathrm{CO}_{2}$ isotherms at $25{ }^{\circ} \mathrm{C}$ for HKUST-1 pellet with different 
$\mathrm{H}_{2} \mathrm{O}$ loadings. Curves are multi temperature Toth equation fits.

In Figure 38, it can be seen that the $\mathrm{CO}_{2}$ capacities for $\mathrm{Ni} / \mathrm{DOBDC}$ decrease when $\mathrm{H}_{2} \mathrm{O}$ molecules are present. No enhancement for $\mathrm{CO}_{2}$ adsorption by $\mathrm{H}_{2} \mathrm{O}$ was found for this MOF sample. The $\mathrm{Ni} / \mathrm{DOBDC}$ sample retained a substantial $\mathrm{CO}_{2}$ capacity at $0.1 \mathrm{~atm}$, about $2.0 \mathrm{~mol} / \mathrm{kg}$, even with a $3.4 \mathrm{~mol} / \mathrm{kg} \mathrm{H} \mathrm{H}_{2} \mathrm{O}$ loading at room temperature. Similar to the case of HKUST-1, the Ni/DOBDC sample could adsorb only small amounts of $\mathrm{CO}_{2}$ for a high $\mathrm{H}_{2} \mathrm{O}$ loading, which here is $28.7 \mathrm{~mol} / \mathrm{kg}(\mathrm{RH} \approx 89 \%)$. In Figure 39, $\mathrm{CO}_{2}$ capacities for $\mathrm{Mg} / \mathrm{DOBDC}$ decrease when the $\mathrm{H}_{2} \mathrm{O}$ loading increases. The $\mathrm{Mg} / \mathrm{DOBDC}$ pellet can adsorb $2.13 \mathrm{~mol} / \mathrm{kg} \mathrm{CO}$ at $0.1 \mathrm{~atm}$, even with a $3.4 \mathrm{~mol} / \mathrm{kg} \mathrm{H} \mathrm{H}_{2} \mathrm{O}$ loading at room temperature. Similarly, the $\mathrm{Mg} / \mathrm{DOBDC}$ sample could adsorb only small amounts of $\mathrm{CO}_{2}$ for a high $\mathrm{H}_{2} \mathrm{O}$ loading.

We also compared the $\mathrm{H}_{2} \mathrm{O}$ effects on $\mathrm{CO}_{2}$ adsorption for the three MOF pellets with those for $5 \mathrm{~A}$ and $\mathrm{NaX}$ zeolite pellets. The results are shown in Figure 40. It is obvious that $\mathrm{H}_{2} \mathrm{O}$ does not inhibit $\mathrm{CO}_{2}$ adsorption for HKUST-1 and Ni/DOBDC as much as it does for $5 \mathrm{~A}$ and $\mathrm{NaX}$ zeolites, as indicated by the less steep slopes for HKUST-1 and $\mathrm{Ni} / \mathrm{DOBDC}$ in the comparison plots. The slope for $\mathrm{Mg} / \mathrm{DOBDC}$ is comparable to zeolites. However, the $\mathrm{CO}_{2}$ capacities of $\mathrm{Mg} / \mathrm{DOBDC}$ at $0.1 \mathrm{~atm}$ are higher than those of zeolites. The two DOBDC series MOFs can adsorb relatively more $\mathrm{CO}_{2}$ than zeolites can before they are saturated with $\mathrm{H}_{2} \mathrm{O}$. Moreover, $\mathrm{H}_{2} \mathrm{O}$ molecules are easier to remove from MOF samples than from zeolites by regeneration as evidenced by lower isotherm slopes

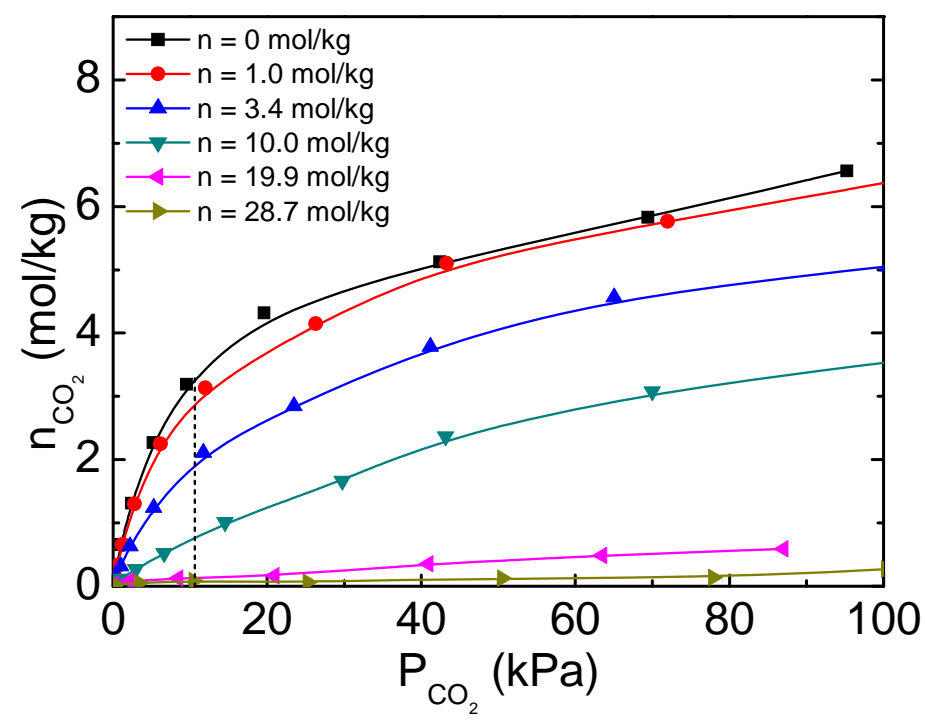

Figure 38. $\mathrm{CO}_{2}$ isotherms at $25^{\circ} \mathrm{C}$ for Ni/DOBDC pellet with different $\mathrm{H}_{2} \mathrm{O}$ loadings. Curves are multi temperature Toth equation fits. 


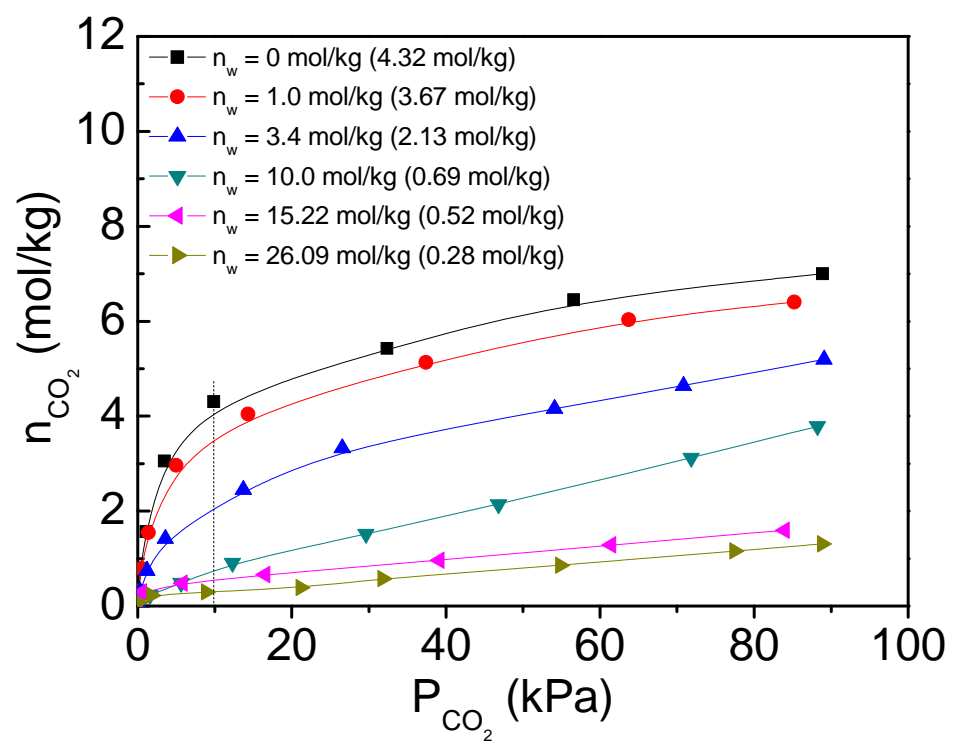

Figure 39. $\mathrm{CO}_{2}$ isotherms at $25^{\circ} \mathrm{C}$ for $\mathrm{Mg} / \mathrm{DOBDC}$ pellet with different $\mathrm{H}_{2} \mathrm{O}$ loadings. Curves are multi temperature Toth equation fits.

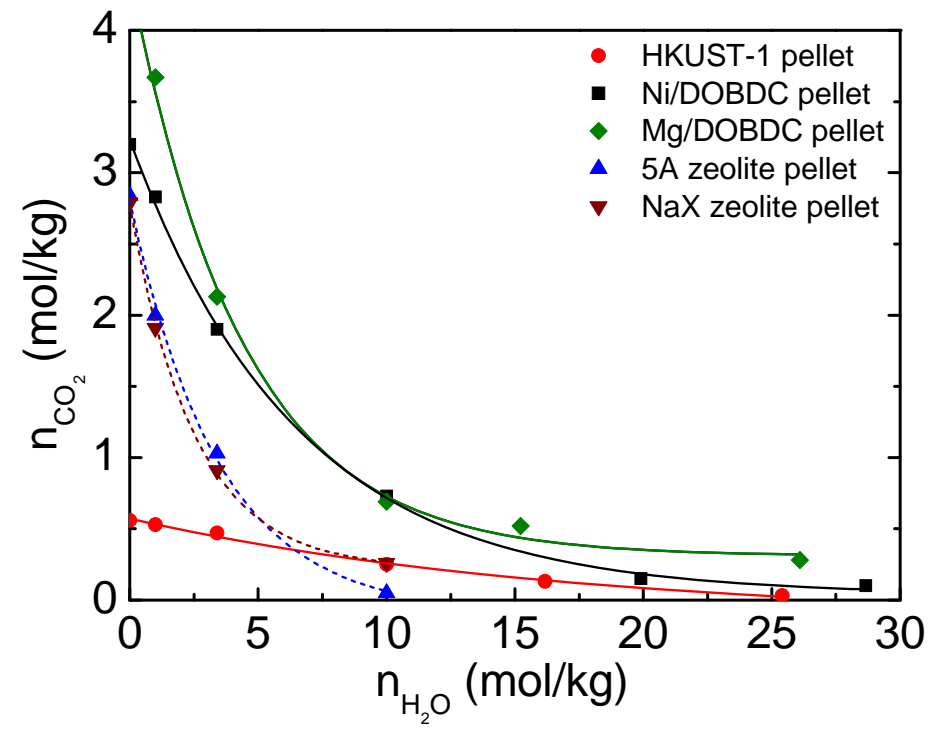

Figure 40. $\mathrm{H}_{2} \mathrm{O}$ effects on $\mathrm{CO}_{2}$ adsorption for $\mathrm{MOF}$ and zeolite pellets at $25{ }^{\circ} \mathrm{C}$. Data points are $\mathrm{CO}_{2}$ capacities at $0.1 \mathrm{~atm}$ for different samples at various $\mathrm{H}_{2} \mathrm{O}$ loadings, and lines are guides for the eyes.

for MOFs at low loadings. ${ }^{67}$ The temperature we used to regenerate the MOF samples in situ is no higher than $200{ }^{\circ} \mathrm{C}$, while $350{ }^{\circ} \mathrm{C}$ or higher is often needed for removing water from zeolites. Further, framework alumina in zeolites can be extracted and react with $\mathrm{CO}_{2}$ in water-saturated streams to form difficult to decompose carbonate species. Besides the comparable $\mathrm{CO}_{2}$ capacity between the two DOBDC MOFs and the 
benchmark zeolites at $0.1 \mathrm{~atm}$ and $25{ }^{\circ} \mathrm{C}$, the smaller water effect together with an easier regeneration process continues to suggest that $\mathrm{Ni} / \mathrm{DOBDC}$ and $\mathrm{Mg} / \mathrm{DOBDC}$ have a promising future in $\mathrm{CO}_{2}$ capture from flue gas.

\section{$\underline{\text { Simulated flue gas effects }}$}

Breakthrough experiments on the new gas-flow system (UM). During the project a custom-built gas flow apparatus (see Figure 5) was constructed in-house for studying the effect of humidity on the $\mathrm{CO}_{2}$ capacities of materials in the M/DOBDC series (where $\mathrm{M}$ $=\mathrm{Zn}, \mathrm{Co}, \mathrm{Ni}, \mathrm{Mg}$; DOBDC = 2,5-dioxido-1,4-benzenedicarboxylate). These samples were chosen due to their high $\mathrm{CO}_{2}$ capacities. The major questions to be addressed regard the performance in the presence of water and other contaminants. Water was scrutinized in the greatest detail.

$\mathrm{Zn} / \mathrm{DOBDC}$ was used as a test material for collecting preliminary $\mathrm{N}_{2} / \mathrm{CO}_{2}$ breakthrough curves from the apparatus. The material was prepared by the published method. ${ }^{6}$ A 162 mg sample of the material was packed into a copper column, then loaded onto the new apparatus under helium flow.

The following flow sequence was used:

Table 12. Flow stages for $\mathrm{N}_{2} / \mathrm{CO}_{2}$ breakthrough over $\mathrm{Zn} / \mathrm{DOBDC}$.

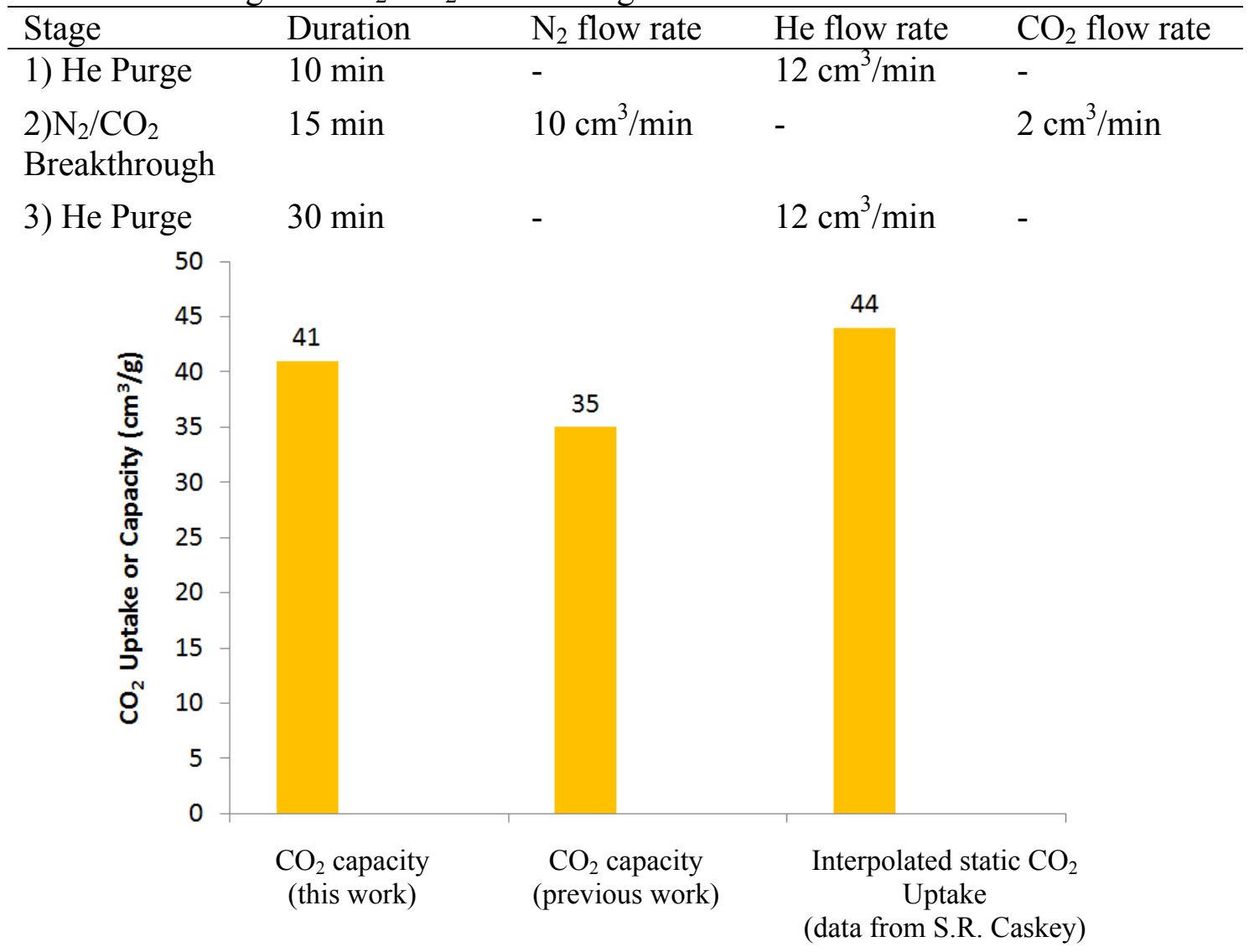

Figure 41. Comparison of $\mathrm{CO}_{2}$ capacities to published static sorption uptake. 
The $\mathrm{CO}_{2}$ capacity of $\mathrm{Zn} / \mathrm{DOBDC}$ was determined from the $\mathrm{CO}_{2}$ breakthrough curve by the previously described method. The average $\mathrm{CO}_{2}$ capacity determined from three runs, was found to be consistent with the $\mathrm{CO}_{2}$ capacity determined from the previous apparatus, and published static $\mathrm{CO}_{2}$ uptake (Figure 41). ${ }^{35}$

$\mathrm{N}_{2} / \mathrm{CO}_{2} / \mathrm{H}_{2} \mathrm{O}$ breakthrough was also performed on $\mathrm{Zn} / \mathrm{DOBDC}$. The $\mathrm{N}_{2}$ stream was humidified by directing the gas into the bubbler and combining the bubbler outlet gas with $\mathrm{CO}_{2}$ in the mixing manifold. The resulting $\mathrm{N}_{2} / \mathrm{CO}_{2} / \mathrm{H}_{2} \mathrm{O}$ gas mixture was charged into a column containing $25 \mathrm{mg}$ of $\mathrm{Zn} / \mathrm{DOBDC}$. The flow stages for the experiment are shown in Table 13, and breakthrough curves are shown in Figure 42.

Table 13. Flow stages for $\mathrm{N}_{2} / \mathrm{CO}_{2} / \mathrm{H}_{2} \mathrm{O}$ breakthrough over $\mathrm{Zn} / \mathrm{DOBDC}$.

\begin{tabular}{lllll}
\hline Stage & Duration & $\mathrm{N}_{2}$ flow rate & He flow rate & $\mathrm{CO}_{2}$ flow rate \\
\hline 1) $\mathrm{He}$ Purge & $10 \mathrm{~min}$ & - & $12 \mathrm{~cm}^{3} / \mathrm{min}$ & - \\
2) $\mathrm{N}_{2} / \mathrm{CO}_{2}$ & $90 \mathrm{~min}$ & $10 \mathrm{~cm}^{3} / \mathrm{min}$ & - & $2 \mathrm{~cm}^{3} / \mathrm{min}$ \\
Breakthrough & & & &
\end{tabular}

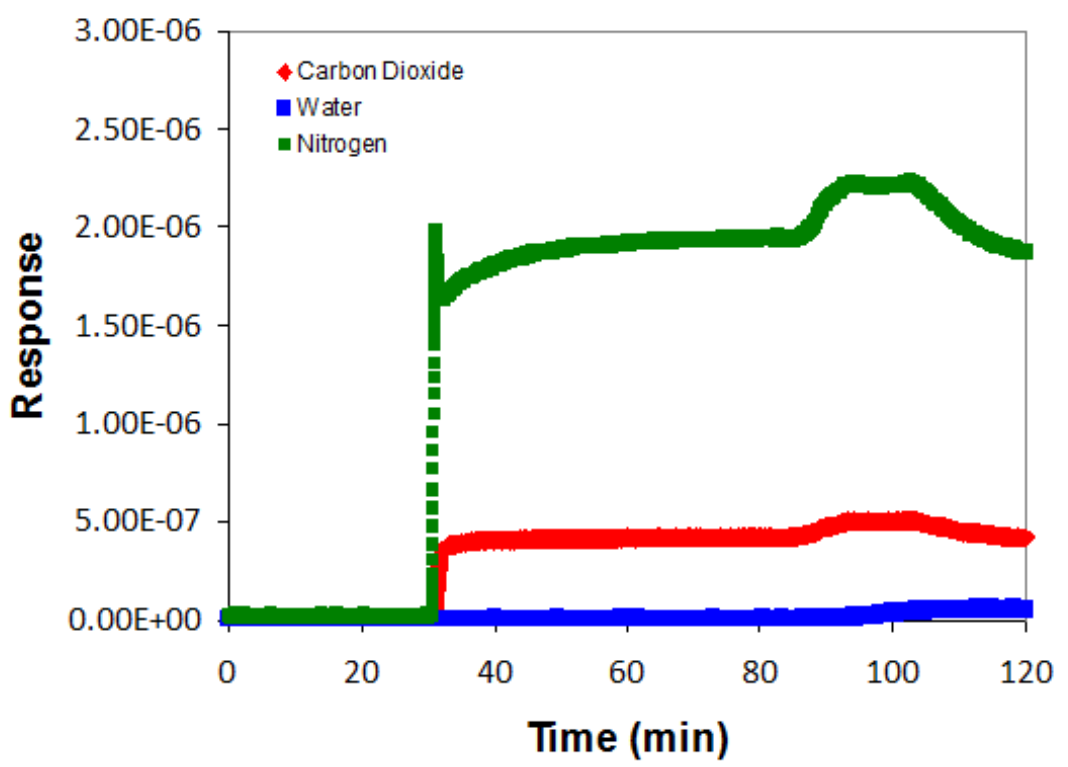

Figure 42. Breakthrough curves for $\mathrm{N}_{2}, \mathrm{CO}_{2}$, and $\mathrm{H}_{2} \mathrm{O}$ over $\mathrm{Zn} / \mathrm{DOBDC}$.

The data show that $\mathrm{H}_{2} \mathrm{O}$ is selectively retained versus $\mathrm{CO}_{2}$ and $\mathrm{N}_{2}$. The slight rise in the $\mathrm{CO}_{2}$ response (beginning at about 85 minutes) is consistent with $\mathrm{H}_{2} \mathrm{O}$ displacing residual $\mathrm{CO}_{2}$ remaining in the $\mathrm{Zn} / \mathrm{DOBDC}$ pores. The simultaneous rise in the $\mathrm{N}_{2}$ response was not expected, and team members at UE suggested that it might be indicative of $\mathrm{H}_{2} \mathrm{O}$ condensate causing a pressure increase in the system. However, $\mathrm{H}_{2} \mathrm{O}$ breakthrough occurred after about 65 minutes, which is consistent with the 2.4 minutes of $\mathrm{H}_{2} \mathrm{O}$ retention per mg of $\mathrm{Zn} / \mathrm{DOBDC}$ determined from a previous $\mathrm{H}_{2} \mathrm{O}$ breakthrough experiment. 
Simulated flow gas studies in a volumetric flow apparatus (VU). Figure 43 shows a schematic diagram of the apparatus used in experiments to study the simulated flue gas conditioning effect. The simulated flue gas used in these experiments is composed of $16 \% \mathrm{CO}_{2}, 100 \mathrm{ppm} \mathrm{SO}, 10 \mathrm{ppm} \mathrm{NO}$, balanced with $\mathrm{N}_{2}$ (the same composition as for the University of Edinburgh). Saturated water vapor at $17{ }^{\circ} \mathrm{C}$ was generated using a water sparger and added to the flow of simulated flue gas. Fixed flow rates of water vapor and simulated flue gas flow through the adsorption bed loaded with a MOF sample. $\mathrm{CO}_{2}$ isotherms were measured for the same sample before and after the simulated flue gas conditioning to reveal the effects of humidity and acid gases on $\mathrm{CO}_{2}$ adsorption performance.

MOF samples were tested for $\mathrm{CO}_{2}$ capacity at $0.1 \mathrm{~atm}$ before and after the simulated flue gas conditioning to reveal the effects of humidity and acid gases on performance. The result for HKUST-1 powder is shown in Figure 44. Generally, the $\mathrm{CO}_{2}$ capacities at 0.1 atm for HKUST-1 powder did not change significantly after being conditioned with different simulated flue gases at room temperature. Note the numerical values shown in the figure for the $\mathrm{CO}_{2}$ loading at the POI. It seems that flue gas at room temperature does

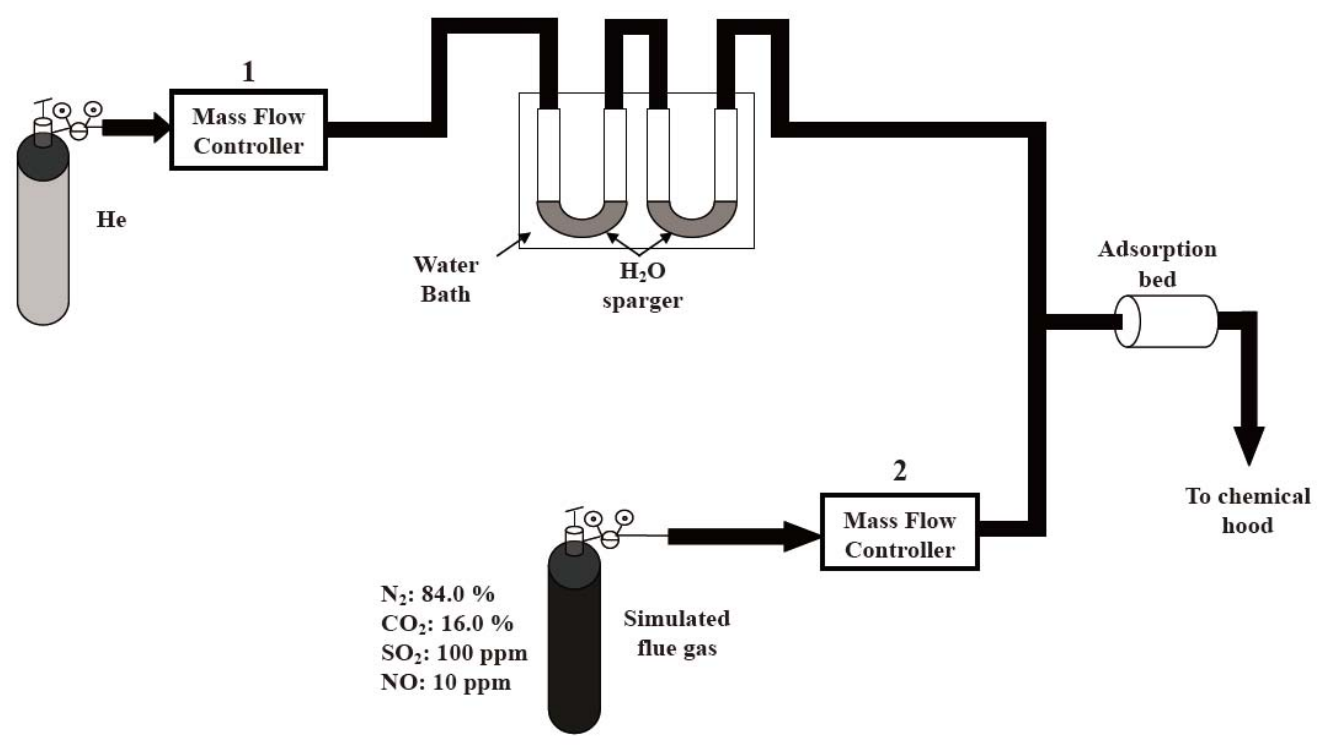

Figure 43. Simulated flue gas conditioning apparatus. 


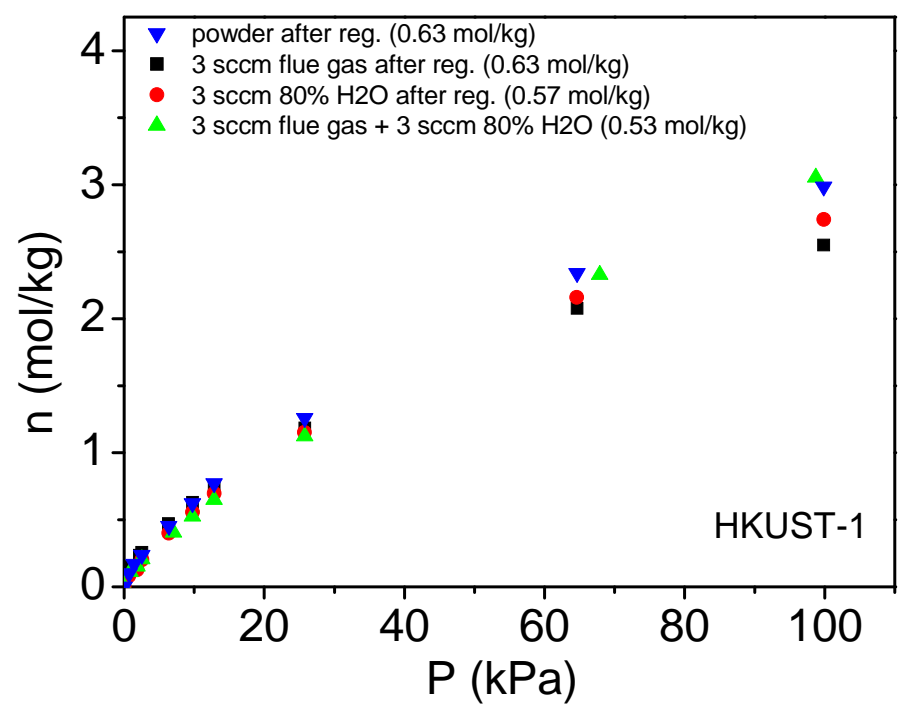

Figure 44. $\mathrm{CO}_{2}$ isotherms at $25^{\circ} \mathrm{C}$ for HKUST-1 pellet before and after different simulated flue gas conditionings.

not affect $\mathrm{CO}_{2}$ adsorption in HKUST-1. However, moisture can impact the simulated flue gas conditioning effect for HKUST-1, as indicated by the reduced capacity of 0.53 $\mathrm{mol} / \mathrm{kg}$ at the POI. Similar results were found for Ni/DOBDC as shown in Figure 45.

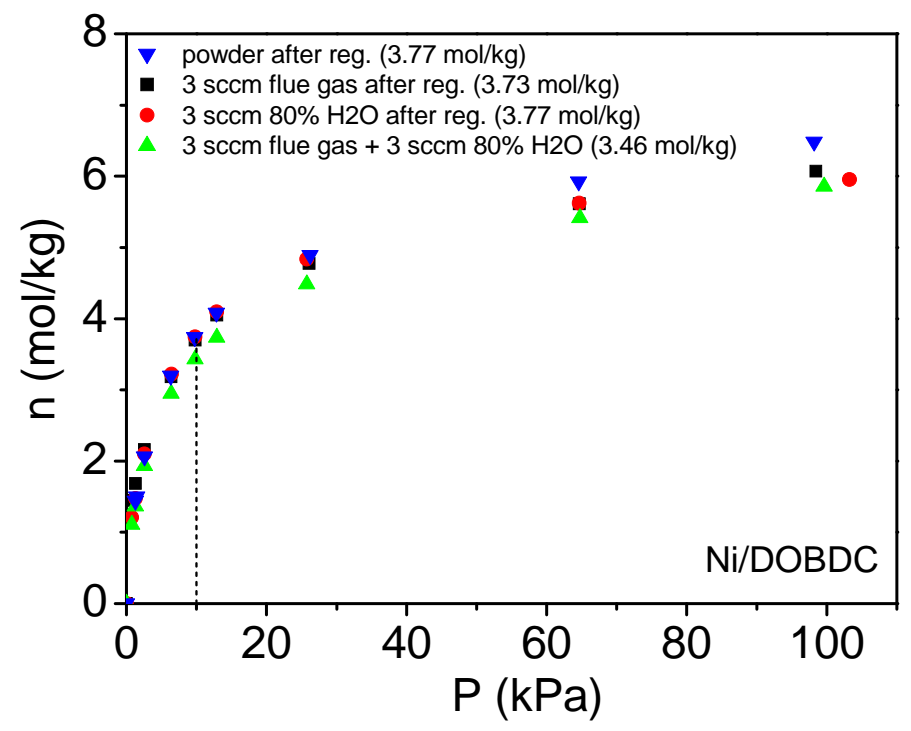

Figure 45. $\mathrm{CO}_{2}$ isotherms at $25{ }^{\circ} \mathrm{C}$ for $\mathrm{Ni} / \mathrm{DOBDC}$ pellet before and after different simulated flue gas conditionings.

ZLC stability test experiments (UE). The final set of experimental tests developed at UE for this project were those in which the stability of the sample to water and impurities, i.e. 
SOx and NOx was evaluated. The tests were carried out on the most promising materials of the DOBDC family. For comparison we also carried out the same tests on 13X zeolite. Since the tests are meant to be with a gas mixture representative of a flue gas, the final formulation of $16 \% \mathrm{v} / \mathrm{v} \mathrm{CO}, 100 \mathrm{ppm} \mathrm{SO}_{2}$ and $10 \mathrm{ppm} \mathrm{NO}$, with the balance of $\mathrm{N}_{2}$ was utilized. Water could not be included, since at high pressure it would have condensed in the cylinder leading to corrosion. This was not a problem, since water can be and was added via the gas dosing system. Oxygen was not included since it is incompatible with $\mathrm{SO}_{2}$, but this will not have a major effect, since in adsorption on MOFs $\mathrm{O}_{2}$ and $\mathrm{N}_{2}$ are likely to have similar properties.

Initially, a sample's stability to water was evaluated. Gas mixtures were prepared with $2000 \mathrm{ppm}$ and $5000 \mathrm{ppm}$ of water. Ni/DOBDC proved to be highly hydrophilic, so in the presence of even low quantities of water, once the sample has reached equilibrium, the adsorbed phase is effectively saturated.

The normal sequence of experimental steps that was carried out on the samples:

1) Dry $\mathrm{CO}_{2}$ capacity measurements.

2) Extended exposure to wet carrier gas and/or $\mathrm{CO}_{2}$. This was meant to evaluate the stability of the sample toward regeneration.

3) Extended exposure to the flue gas as delivered from the pre-mixed cylinder.

4) Extended exposure to wet flue gas (final experiments with $1 \% \mathrm{v} / \mathrm{v}$ water).

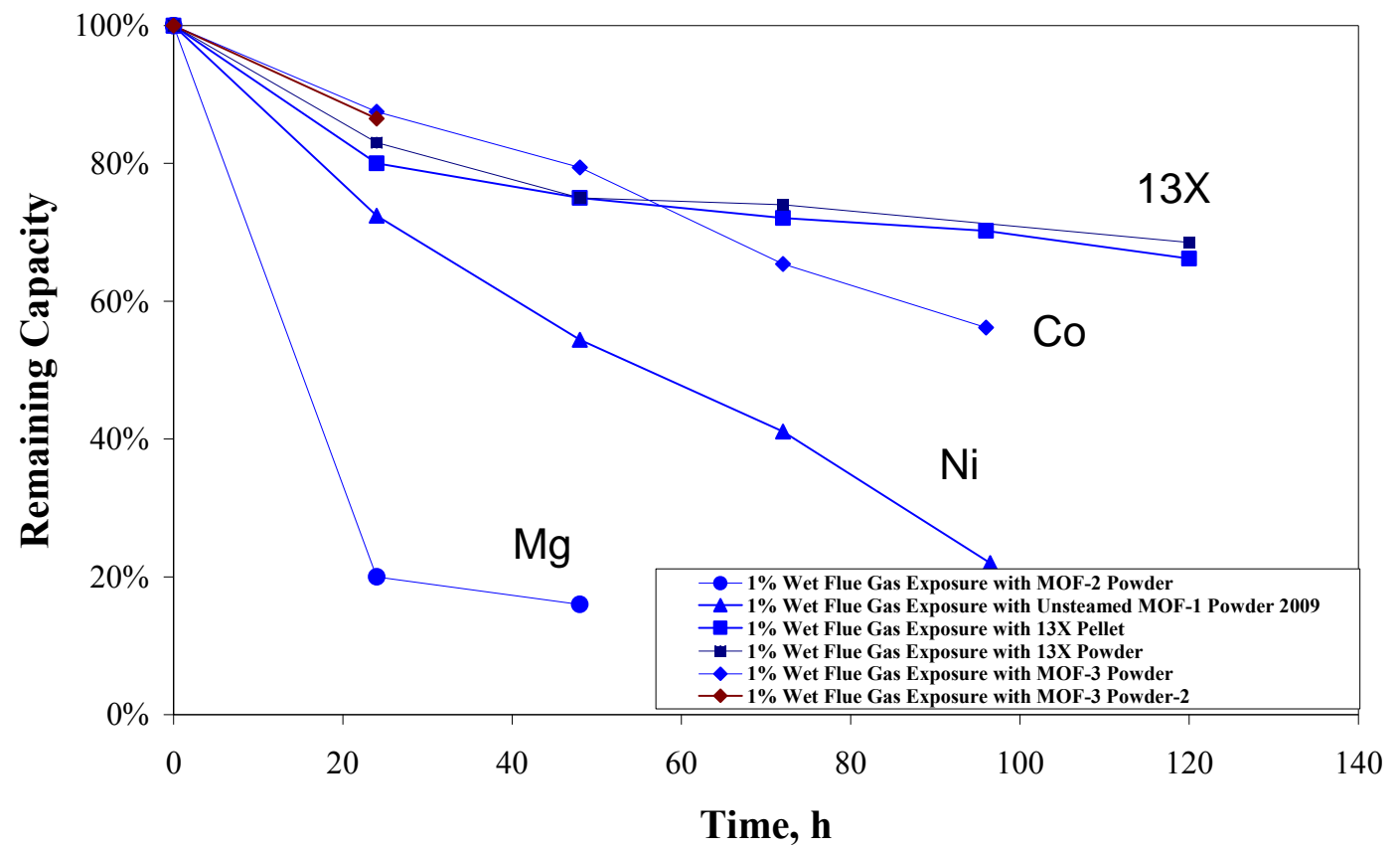

Figure 46. Results of the stability tests with $1 \%$ wet flue gas (step 4).

After each exposure the samples were regenerated and the residual capacity was evaluated. Several samples of Ni/DOBDC were evaluated and all showed very good stability to water (step 1). For step 2, the exposure to the flue gas as delivered by the cylinder, it was realised that for short periods of exposure, the materials were stable, but 
for prolonged exposures there was a clear loss in capacity. By monitoring the outlet gas continuously during these experiments, it was determined that the synthetic flue gas contains trace amounts of water, which accumulate in the adsorbent. It is therefore not possible to test the sample with a truly 'dry' flue gas, since adding a gas drier would mean that both $\mathrm{SO}_{2}$ and $\mathrm{NO}$ would be adsorbed as well, reverting to a $\mathrm{CO}_{2} / \mathrm{N}_{2}$ mixture being fed to the ZLC. The ranking in terms of stability to the wet flue gas follows the inverse order of the capacity for $\mathrm{CO}_{2}$, i.e. $\mathrm{Co}>\mathrm{Ni}>\mathrm{Mg}$. What was interesting from these results is the fact that the three materials behave very differently in terms of stability, with Co/DOBDC showing a very peculiar 'switch' after the first exposure. $13 \mathrm{X}$ zeolite showed some loss of capacity after the first exposure, followed by a slow secondary decay.

The Ni/DOBDC sample showed excellent stability to water, with full regeneration at 125 ${ }^{\circ} \mathrm{C}$. When we tested the samples with wet flue gas there was a progressive loss of capacity, which can be seen in Figure 47.

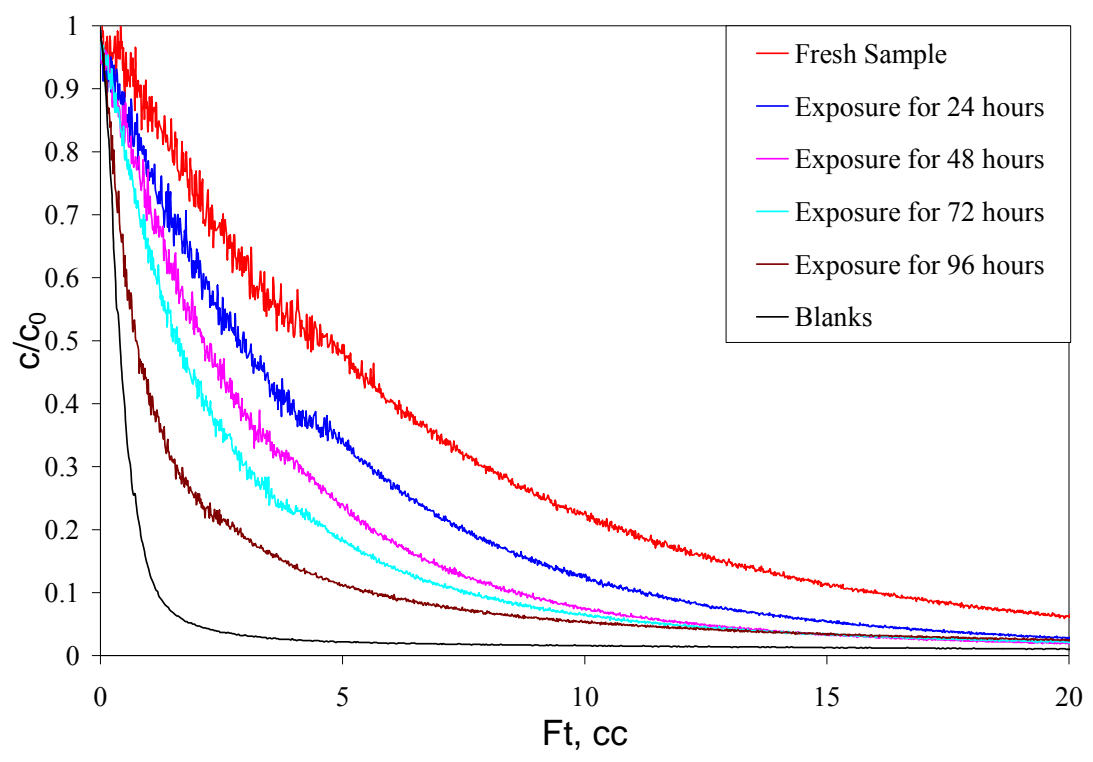

Figure 47. Ni/DOBDC stability results after exposure to $1 \% \mathrm{v} / \mathrm{v}$ wet flue gas.

What is interesting to note from the stability results is that the capacity loss seems to be uniform, i.e. at each extended exposure more material is deactivated but the general shape of the isotherm and of the ZLC response remains the same.

The behaviour of the Co/DOBDC sample is significantly different from that of the $\mathrm{Ni}$ one. What is interesting in this case is the fact that the shape of the isotherm changes. The effect of the wet flue gas seems to make the adsorption sites stronger, so the isotherm after the first treatment shifts to a non-linear one, as can be seen in Figure 48. 


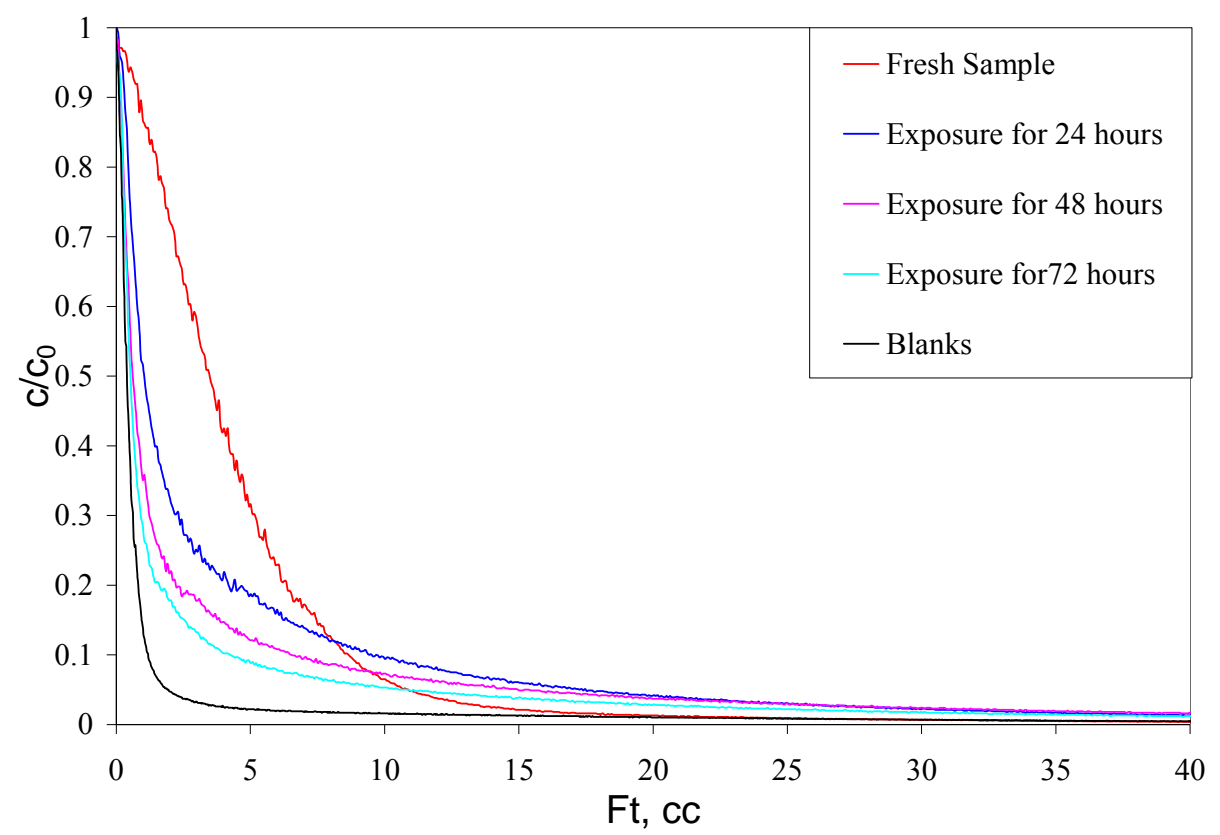

Figure 48. Co/DOBDC stability results after exposure to $1 \% \mathrm{v} / \mathrm{v}$ wet flue gas.

In order to understand this better, the UE team analysed what was happening during the exposure to the flue gas. Figure 49 shows the MS traces for the fresh sample.

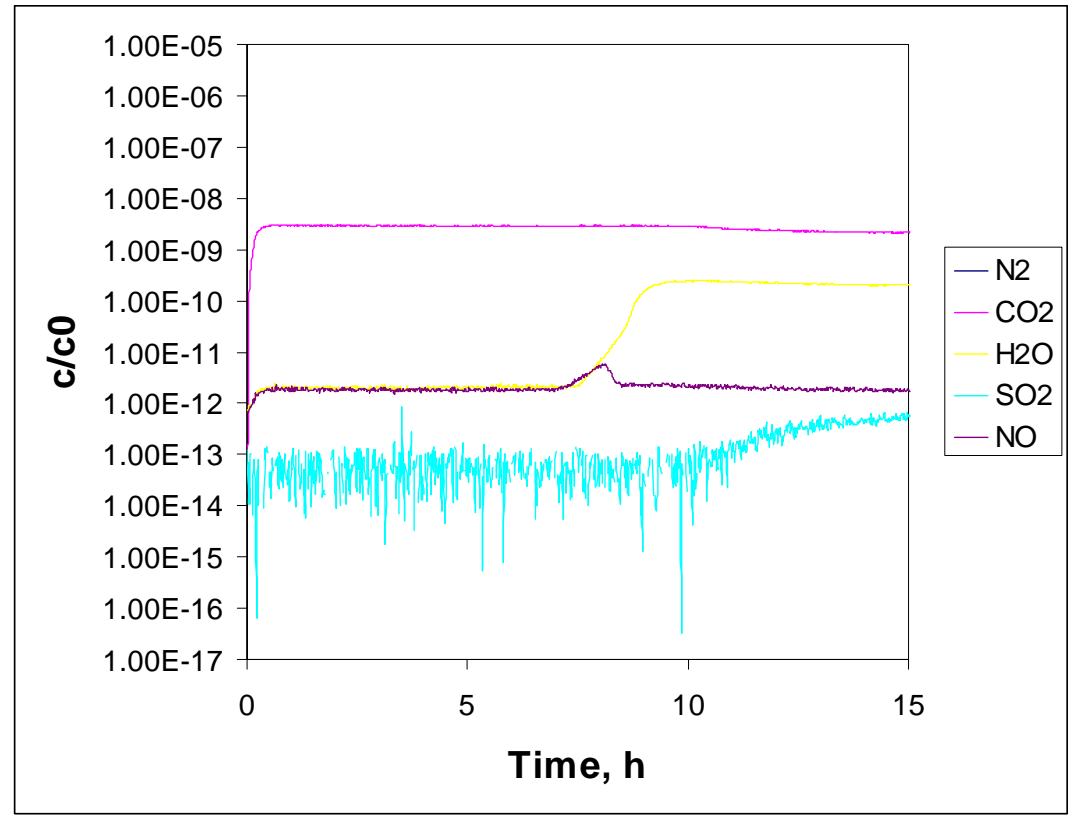

Figure 49. MS trace for different components on fresh sample of $\mathrm{Co} / \mathrm{DOBDC}$.

The ZLC is behaving like a normal breakthrough apparatus in this case. What is important is to compare the $\mathrm{SO}_{2}$ signal for the first treatment and for the second and subsequent ones (the second is shown in Figure 50). 


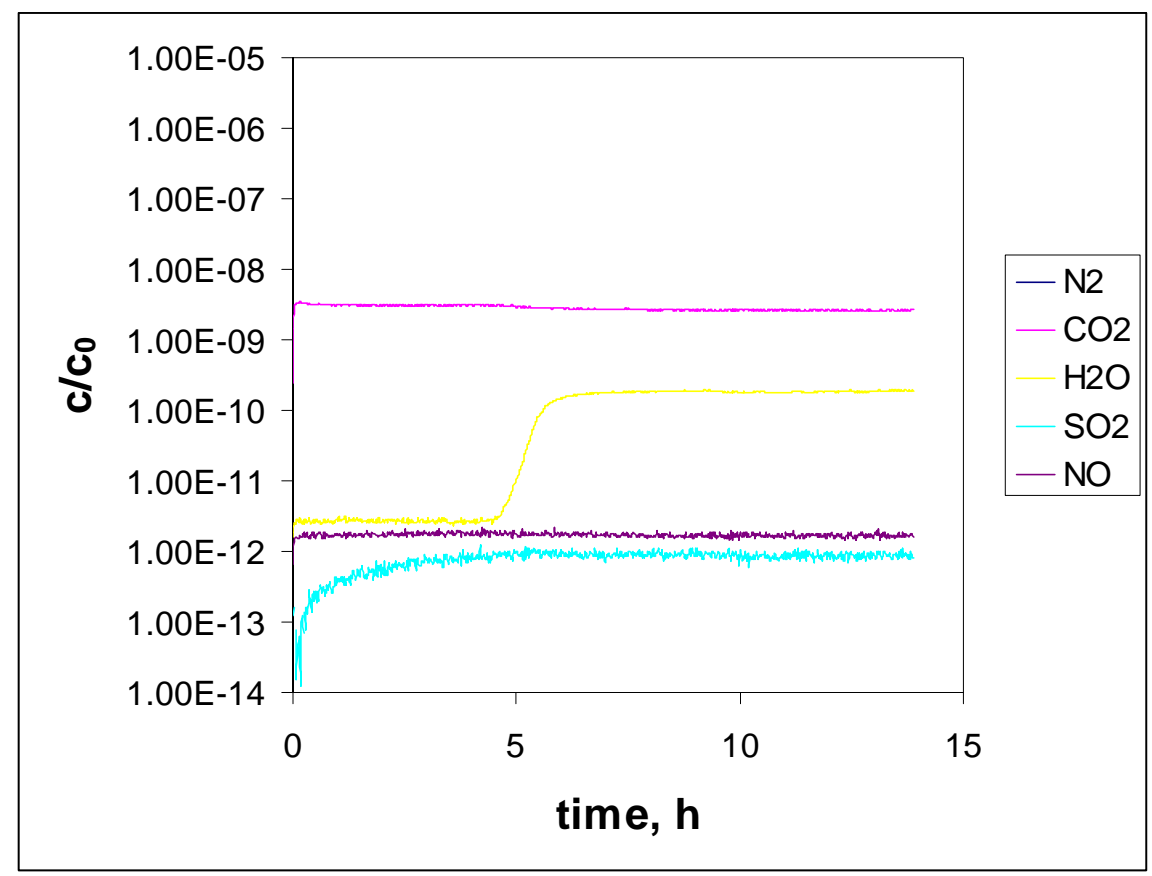

Figure 50. MS trace for different components on second exposure of Co sample.

The $\mathrm{SO}_{2}$ in the first exposure is clearly adsorbed in the Co/DOBDC sample, while it shows an immediate breakthrough in the second case. It seems that the sample changes the pore openings and does not allow $\mathrm{SO}_{2}$ to diffuse into the solid after the first treatment. Or perhaps only a portion of the MOF's UMCs are titrated with $\mathrm{SO}_{2}$ irreversibly. Either or a combination of these effects would explain why subsequent treatments only induce minor reductions in the $\mathrm{CO}_{2}$ capacity.

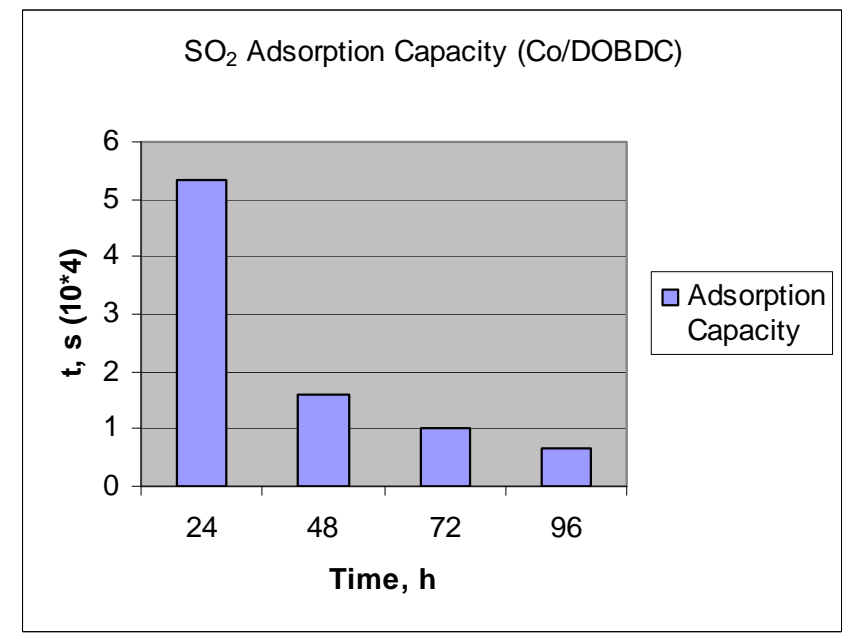

Figure 51. $\mathrm{SO}_{2}$ capacity over $24 \mathrm{hr}$ exposure at subsequent cycles - Co.

In the future, this phenomenon will need to be examined more carefully because if the stability behaviour is linked to a subtle structural change, it could be that the shape of the ZLC curve is affected by this as well. This could mean a mass transfer limitation. 
Figure 51 shows the first moments (mean residence time) of $\mathrm{SO}_{2}$ on the $\mathrm{Co} / \mathrm{DOBDC}$ sample, clearly indicating a very sharp drop after the first cycle. By contrast Figure 52 shows the same calculation for the Ni/DOBDC material.

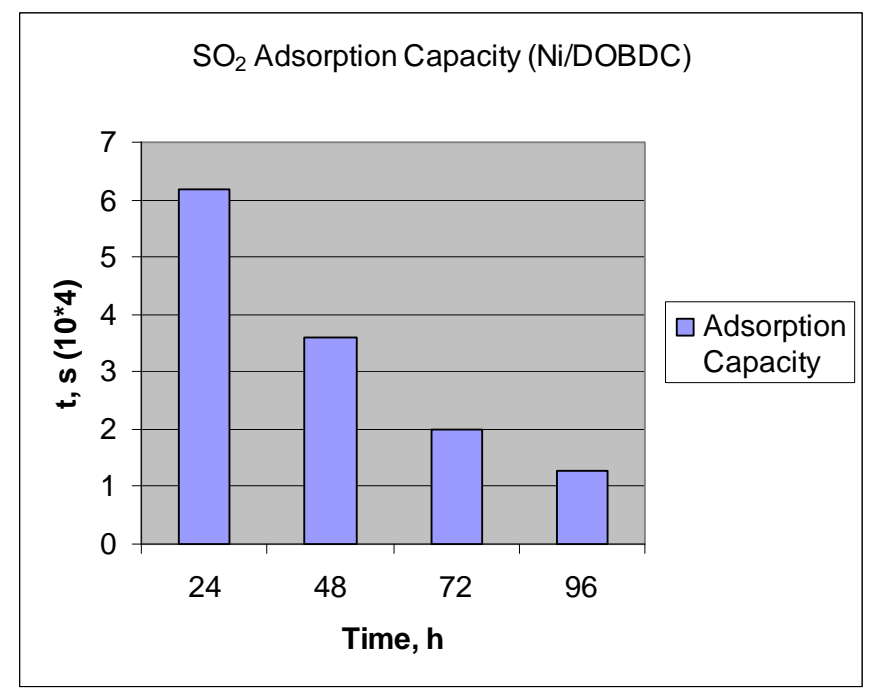

Figure 52. $\mathrm{SO}_{2}$ capacity over $24 \mathrm{~h}$ exposure at subsequent cycles for Ni/DOBDC.

In the case of $\mathrm{Ni} / \mathrm{DOBDC}$, the $\mathrm{SO}_{2}$ capacity follows a similar regular trend as for the $\mathrm{CO}_{2}$ capacity.

\section{Mg/DOBDC Stability Results.}

Figure 53 shows the results for the $\mathrm{Mg} / \mathrm{DOBDC}$ sample.

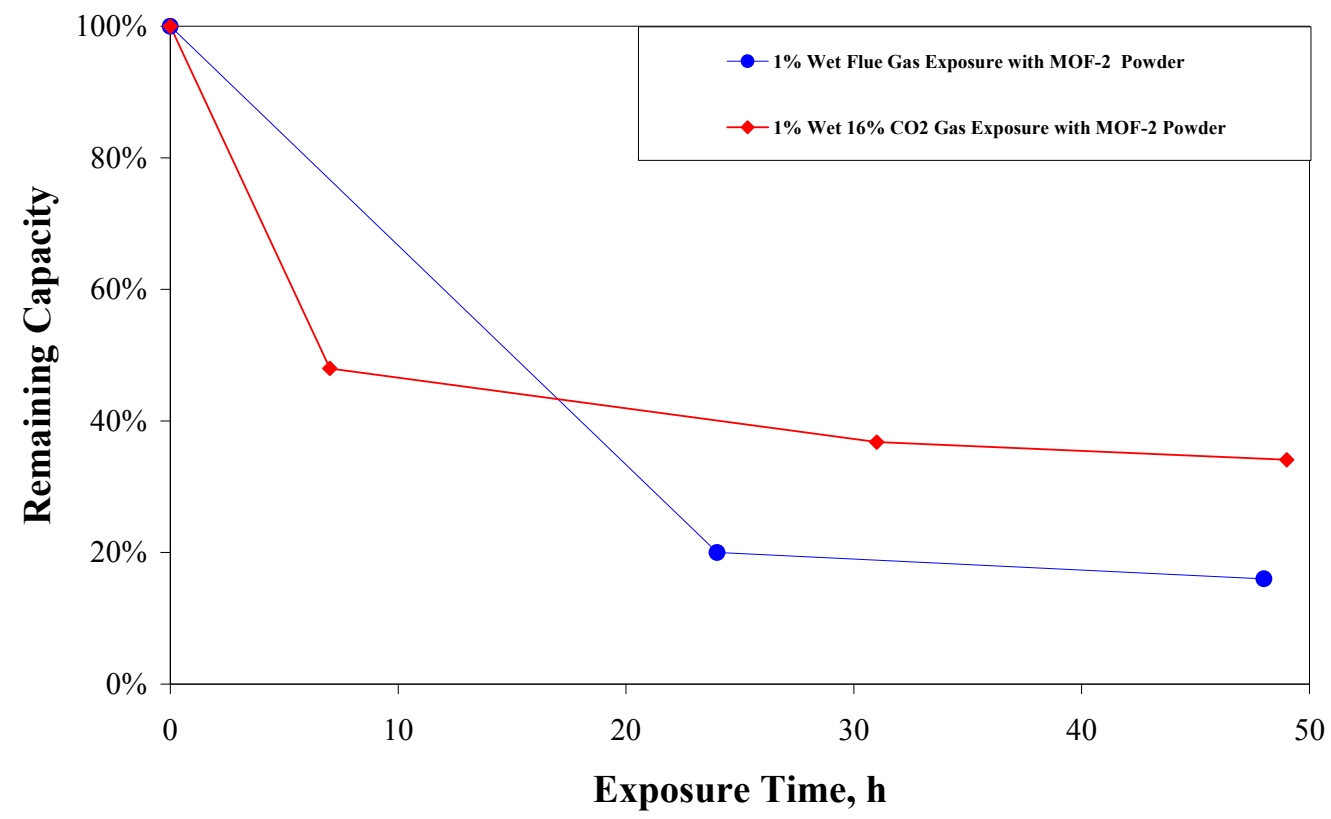

Figure 53. Water and wet flue gas stability for $\mathrm{Mg} / \mathrm{DOBDC}$ sample. 
In this case, a shorter initial exposure time of 6 hours to try and capture the initial rate was also carried out. There is an additional effect due to the flue gas, but it is clear that in this case most of the deactivation is due to the effect of water. For this sample the shape of the ZLC curve does not change qualitatively.

The ZLC columns treated with the flue gas were sent to UOP for further characterisation of the solids and the tests broadly confirm the $\mathrm{CO}_{2}$ capacity results, i.e. where significant changes are observed XRD patterns show significant changes in the crystal structure. That is, $\mathrm{Mg} / \mathrm{DOBDC}$ powder samples were analyzed by $\mathrm{x}$-ray diffraction (XRD) to determine the purity of the freshly activated sample as well as the damage caused by the high severity steaming as described above. As can be seen in Figure 54, the steaming process reduced the intensity of the diffraction peaks significantly. This explains why these samples did not adsorb as much $\mathrm{CO}_{2}$ as the freshly activated sample.

The ZLC system appears to be extremely useful when trying to accelerate the deactivation of the samples due to SOx and NOx impurities. The fact that the treatment can be repeated in situ, in a relatively simple way using a very small sample, has shown that the technique is a key tool in the screening of novel adsorbents for carbon capture applications.

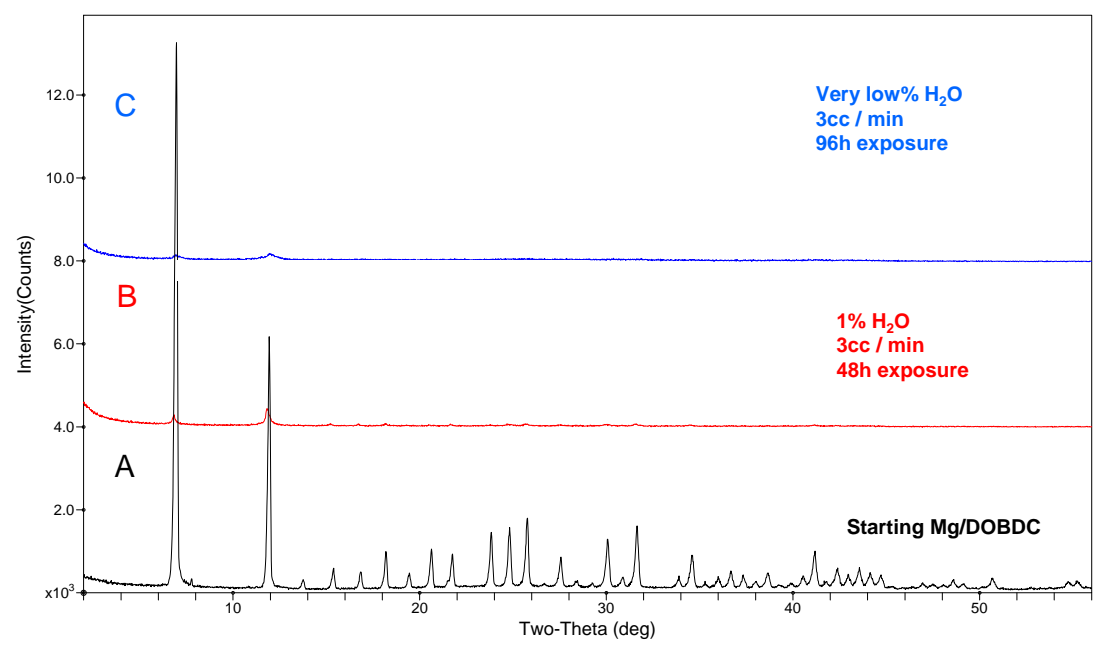

Figure 54. XRD spectra for $\mathrm{Mg} / \mathrm{DOBDC} 2010$ freshly activated (A), exposed to $1 \%$ water at $25{ }^{\circ} \mathrm{C}$ for $24 \mathrm{~h}(\mathrm{~B})$, and exposed to $<<1 \%$ water at $25{ }^{\circ} \mathrm{C}$ for $96 \mathrm{~h}(\mathrm{C})$.

\subsection{The MOF-based vPSA process concept}

The team's ultimate goal was to be able to design a process for $\mathrm{CO}_{2}$ removal from flue gas using the optimized MOF materials and process parameters identified in Phase 3 . A more detailed account of this design is provided in Appendix 2 as a separate "Process 
Design and Economics Analysis Report." This Report was submitted to the NETL Project Manager in August 2010. The Report includes a high level design of a MOFbased vPSA process for removal of $\mathrm{CO}_{2}$ from flue gas using $\mathrm{Mg} / \mathrm{DOBDC}$, including pretreatment requirements and process conditions.

\subsection{Pilot Study Design}

A pilot study to demonstrate $\mathrm{CO}_{2}$ removal from the slipstream of an electric powergeneration plant was designed and documented in a "Pilot Study Design Report" in Appendix 3.

\subsection{Economic Analyses}

The economics of the proposed process were analyzed, using the parameters developed in the material testing and process concept. The economic analysis was performed in accordance with the guidelines set forth in NETL's Carbon Capture and Sequestration Systems Analysis Guidelines. The results were documented in the "Process Design and Economic Analysis Report" in Appendix 2. The bottom line is that a MOF-based $v P S A$ CC process has a COE of $65 \%$.

\subsection{Commercialization Study Update}

UOP reviewed feedback on the original commercialization study prepared in 2009 to evaluate whether or not the study has changed sufficiently to effect an adequate assessment of the technology and its commercial potential. The study was updated and appears as Appendix 4. 


\section{FINAL MILESTONE STATUS}

\begin{tabular}{|c|c|c|c|c|}
\hline & Milestone & $\begin{array}{c}\text { Planned } \\
\text { Completion Date }\end{array}$ & $\begin{array}{c}\text { Actual } \\
\text { Completion Date }\end{array}$ & Comment \\
\hline 1 & Refined performance targets and material focus & $6 / 30 / 2007$ & $5 / 14 / 2007$ & on time \\
\hline 2 & $\begin{array}{l}\text { Synthesis of up to } 50 \text { known MOF materials in } \\
\text { sufficient quantities for stability and performance } \\
\text { testing }\end{array}$ & $3 / 31 / 2008$ & $3 / 31 / 2008$ & $\begin{array}{c}\text { Over } 35 \text { samples prepared and } \\
\text { evaluated. }\end{array}$ \\
\hline 3 & $\begin{array}{l}\mathrm{CO} 2 \text { adsorption capacity measurements confirmed at } \\
\text { higher temperature for materials with demonstrated } \\
\text { hydrothermal stability and low temperature capacity }\end{array}$ & $3 / 31 / 2008$ & $3 / 31 / 2008$ & Demostrated for several MOFs. \\
\hline 4 & $\begin{array}{l}\text { Up to } 10 \mathrm{MOF} \text { materials selected for further } \\
\text { development and testing }\end{array}$ & $6 / 30 / 2008$ & $6 / 3 / 2008$ & See text for list of Top Ten MOFs. \\
\hline 5 & $\begin{array}{l}\mathrm{CO} 2 \text { capacity in the presence of } \mathrm{H} 2 \mathrm{O} \text { measured at two } \\
\text { temperatures on up to } 10 \text { materials passing Year } 1 \\
\text { capacity and hydrothermal stability screens. }\end{array}$ & $9 / 30 / 2008$ & $9 / 30 / 2008$ & NilDOBDC complete \\
\hline 6 & $\begin{array}{l}\mathrm{CO} 2 \text { capacity and hydrothermal stability of up to } 5 \\
\text { formed materials validated against powders. }\end{array}$ & $3 / 31 / 2009$ & $3 / 27 / 2009$ & $\begin{array}{l}\text { Formed Ni DOBDC lost only } 10 \% \\
\text { capacity. }\end{array}$ \\
\hline 7 & $\begin{array}{l}\text { Demonstrate improved material(s) meeting Year } 2 \\
\text { performance target, including contaminant tolerance. }\end{array}$ & $3 / 31 / 2009$ & $3 / 31 / 2009$ & $\begin{array}{l}\text { Six MOF materials met this } \\
\text { requirement }\end{array}$ \\
\hline 8 & $\begin{array}{l}\text { At least one optimized product prepared at sufficient } \\
\text { scale for pilot testing and its manufacturing cost } \\
\text { estimated. }\end{array}$ & $9 / 30 / 2009$ & $8 / 24 / 2010$ & $\begin{array}{l}\text { Synthesis variables determined for } \\
\mathrm{Mg} / \text { and Ni/DOBDC. Cost } \\
\text { estimate: about } \$ 100 \text { /pound }\end{array}$ \\
\hline 9 & $\begin{array}{l}\text { Detailed performance testing complete on optimized } \\
\text { product. }\end{array}$ & $12 / 31 / 2009$ & $6 / 30 / 2010$ & Delayed by two quarters. \\
\hline 10 & Pilot study design complete. & $3 / 31 / 2010$ & $9 / 28 / 2010$ & Delayed by two quarters. \\
\hline 11 & Economic analysis complete. & $3 / 31 / 2010$ & $8 / 24 / 2010$ & Delayed by two quarters. \\
\hline
\end{tabular}

All Milestones except 9, 10 and 11 were completed on time. The roughly two quarter delay for Milestones 9, 10 and 11 had no negative effects on the overall Project. 


\section{CONCLUSIONS}

(1) The DOBDC series of MOFs with unsaturated metal centers in their structures are very good for $\mathrm{CO}_{2}$ adsorption, especially in the low pressure region.

(2) A virtual high-throughput screening model was developed at NU and used to screen MOFs for removal of $\mathrm{CO}_{2}$ from flue gas. The model was validated against experimental adsorption isotherms, and the team found that below 1 bar $\mathrm{CO}_{2}$ uptake correlates well with the enthalpy of adsorption and the best MOFs for removal of $\mathrm{CO}_{2}$ from flue gas have a high density of open metal sites.

(3) An optimized Mg/DOBDC sample provided by UM outperformed all MOF and zeolite materials evaluated to date, as measured at $\mathrm{VU} . \mathrm{CO}_{2}$ adsorption modeling results from NU suggest that modified versions of $\mathrm{Mg} / \mathrm{DOBDC}$ could perform even better. (4) Synthesis yields of many MOFs, including Ni/DOBDC, have been improved significantly at UOP and at UM. It is necessary to scale up the production of the DOBDC series of MOFs further in order to make them economically available for their application in $\mathrm{CO}_{2}$ capture from flue gas. Economies of scale should help to reduce raw materials costs.

(5) Electrostatic interactions were found to be important for proper modeling of $\mathrm{CO}_{2}$ adsorption in MOFs. Using Monte Carlo simulations we discovered that a low loading of water molecules, bound to coordinatively unsaturated metal centers, can increase $\mathrm{CO}_{2}$ adsorption significantly at low pressures in the MOF HKUST-1. This prediction was later verified experimentally, providing additional confidence in the modeling.

(6) Breakthrough testing in dry simulated flue gas generated a ranking of MOFs with high $\mathrm{CO}_{2}$ capacity that matches gravimetric and ZLC experimental rankings.

(7) Kinetic measurements on pellets and pieces of pellets suggest that the pellets are uniform and that, as expected, that mass transport is macropore diffusion controlled. (8) Initial water isotherm measurements on Ni/DOBDC and several other MOFs at UOP suggest that some of the other MOFs are more tolerant to water vapor exposure.

(9) We learned that initial sample activation is critical to removing water adsorbed in the MOF pores, and that we must keep MOFs such as Ni/DOBDC free from moisture in service.

(10) A small amount of water did not decrease, and may actually increase the $\mathrm{CO}_{2}$ capacity of HKUST-1. Water does not affect $\mathrm{CO}_{2}$ adsorption on HKUST-1 and $\mathrm{Ni} / \mathrm{DOBDC}$ as much as on $5 \mathrm{~A}$ and $\mathrm{NaX}$ zeolites, and water would be more easily removed from the MOFs by regeneration.

(11) The Team achieved the end Phase 2 performance target of " $15 \mathrm{wt} \% \mathrm{CO}_{2}$ capacity at $20{ }^{\circ} \mathrm{C}$ at up to 2 atmospheres pressure (also see Table 2 above), with a minimum stability of $75 \% \mathrm{CO}_{2}$ capacity after exposure of material to 5 mole $\%$ steam at $100{ }^{\circ} \mathrm{C}$ for 2 hours. These targets were achieved for formed Ni/DOBDC pellets. We noted that the $\mathrm{CO}_{2}$ capacity at the point of interest (POI) for the steamed Ni/DOBDC powder decreases about $8 \%-12 \%$ comparing with those of the unsteamed powder, while the $\mathrm{CO}_{2}$ capacity at the POI for the unsteamed pellet decreases about $10 \%-13 \%$ in comparison with the unsteamed powder.

(12) Unfortunately, Ni/DOBDC fully equilibrated with water does not pick up appreciable $\mathrm{CO}_{2}$. This means the flue gas stream would need to be dried before being passed over an adsorbent bed of Ni/DOBDC. 
(13) $\mathrm{Mg} / \mathrm{DOBDC}$ lacks cyclic stability without thermally reactivating open metal sites on the MOF.

(14) The ZLC system appears to be extremely useful when trying to accelerate the deactivation of the samples due to $\mathrm{SO}_{\mathrm{x}}$ and $\mathrm{NO}_{\mathrm{x}}$ impurities. The fact that the treatment can be repeated in situ, in a relatively simple way using a very small sample, has shown that the technique is a key tool in the screening of novel adsorbents for carbon capture applications.

(15) End Year 3 stability performance targets of retention of up to $75 \%$ of $\mathrm{CO}_{2}$ capacity after exposure to up to $15 \mathrm{~mol} \%$ steam at $150{ }^{\circ} \mathrm{C}$ for four hours on Ni/DOBDC pellets and extrudates was not achieved. Only about $45 \%$ capacity was retained. (Note that in the actual application, the MOF adsorbent will never be exposed to such harsh steaming.) Nevertheless, the material was not completely destroyed by the extreme steaming conditions. This suggests that MOFs still hold great promise in this application provided they are protected from moisture.

(16) In the practical applications, a guard bed loaded with silica gel or alumina can be used to remove water in flue gas and to protect the high $\mathrm{CO}_{2}$ capacities for the DOBDC series of MOFs.

(17) We found that a MOF-based vPSA process is a less expensive option than using amines to capture $\mathrm{CO}_{2}$. 


\section{REFERENCES}

${ }^{1}$ Chae, H.K.; Siberio-Perez, D.Y.; Kim, J.; Go, Y; Eddaoudi, M.; Matzger, A.J.; O’Keefe, M.; Yaghi, O.M. Nature 2004, 427, 523.

2 Li, H.; Eddaoudi, M.; O’Keefe, M.; Yaghi, O.M. Nature 1999, 402, 276.

${ }^{3}$ Huang, L.; Wang, H.; Chen, J.; Wang, Z.; Sun, J.; Zhao, D.; Yan, Y. Micropor. Mesopor. Mater. 2003, $58,105$.

${ }^{4}$ Rosi, N.L.; Kim, J.; Eddaoudi, M.; Chen, B.; O’Keefe, M.; Yaghi, O.M. J. Am. Chem. Soc. 2005, 127, 1504.

${ }^{5}$ Chen, B.; Liang, C.; Yang, J.; Contreras, D.S.; Clancy, Y.L.; Lobkovsky, E.B.; Yaghi, O.M.; Dai, S. Angew. Chem. Int. Ed. 2006, 45, 1390.

${ }^{6}$ Rowsell, J.L.C.; Yaghi, O.M., J. Am. Chem. Soc. 2006, 128, 1304-1315.

${ }^{7}$ Loiseau, T.; Serre, C.; Huguenard, C.; Taulelle, G.F.; Henry, M.; Bataille, T.; Ferey, G. Chem. Eur. J. 2004, 10, 1373.

${ }^{8}$ Loiseau, T.; Lecroq, L.; Volkringer, C.; Marrot, J.; Ferey, G.; Haouas, M.; Taulelle, F.; Bourrelly, S.; Llewellyn, P.L.; Latroche, M. J. Am. Chem. Soc. 2006, 128, 10223

${ }^{9}$ Ferey, G.; Mellot-Draznieks, C.; Serre, C.; Millange, F.; Dutour, J.; Surble, S.; Margiolaki, I. Science 2005, 309, 2040.

${ }^{10}$ Ma, S.; Sun, D.; Wang, X.-S.; Zhou, H.-C. Angew. Chem. Int. Ed. 2007, 46, 2458

${ }_{11}$ Park, K.S.; Ni, Z.; Cote, A.P.; Choi, J.Y.; Huang, R.; Uribe-Romo, F.J.; Chae, H.K.; O’Keefe, M.; Yaghi, O.M. Proc. Natl. Acad. Sci. 2006, 103, 10186

${ }^{12}$ Szeto, K.C.; Lillerud, K.P.; Tilset, M.; Bjorgen, M.; Prestipino, C.; Zecchina, A.; Lamberti, C.; Bordiga, S. J. Phys. Chem. B. 2006, 110, 21509

${ }^{13}$ Bourrelly, S., Llewellyn, P.L., Serre, C., Millange, F., Loiseau, T., Ferey, G. J. Am. Chem. Soc. 2005, 127,13519

${ }^{14}$ Liu, Y., Krastov, V.C., Larsen, R., Eddaoudi, M. Chem. Commun. 2006, 1488.

${ }^{15}$ Guo, X., Zhu, G., Li, Z., Sun, F., Yang, Z., Qiu, S. Chem. Commun. 2006, 3172.

${ }^{16}$ Pan, L., Adams, K.M., Hernandez, H.E., Wang, X., Zheng, C., Hattori, Y., Kaneko, K. J. Am. Chem. Soc. 2003, 125, 3062

${ }^{17}$ Dybtsev, D., Chun, H., Yoon, S.H., Kim, D., Kim, K. J. Am. Chem. Soc., 2004, 126, 32.

${ }^{18}$ Eddaoudi, M., Kim, J., Rosi, N., Vodak, D., Wachter, J., O’Keefe, M., Yaghi, O.M. Science 2002, 295, 469-472.

${ }^{19}$ Eddaoudi, M., Kim, J., Vodak,D., Sudik, A., Wachter, J., O’Keefe, M., Yaghi, O.M. Proc. Natl. Acad. Sci. 2002, 99(8), 4900.

${ }^{20}$ Gu, J.-Z., Lu, W.-G., Jiang, L., Zhou, H.-C., Lu, T.-B. Inorg. Chem. 2007, 46, 5835. 
${ }^{21}$ Navarro, J.A.R., Barea, E., Salas, J.M., Masciocchi, N., Galli, S., Sironi, A., Ania, C.O., Parra, J.B. Inorg. Chem. 2006, 45, 2397.

${ }^{22}$ Dietzel, P.D.C., Morita, Y., Blom, R., Fjllvag, H. Angew. Chem. Int. Ed. 2005, 44, 6354; Matzger, A. personal communication.

${ }^{23}$ Dietzel, P.D.C., Panella, B., Hirscher, M., Blom, R., Fjllvag, H. Chem. Commun. 2006, 959.

${ }^{24}$ Volkringer, C., Popov, D., Loiseau, T., Guillou, N., Ferey, G., Haouas, M., Taulelle, F., Mellot-

Draznieks, C., Burghammer, M., Riekel, C. Nature Mater. 2007, 6, 760

${ }^{25}$ Maji, T.K., Matsuda, R., Kitagawa, S. Nature Mater. 2007, 6, 142

${ }^{26}$ Rosi, N. "Design, Synthesis, and Control of Metrics, Functionality, and Interpenetration in MetalOrganic Frameworks and their Application in Hydrogen Storage," PhD thesis, U Michigan 2005

${ }^{27}$ Ma, S., Wang, X.-S., Manis, E.S., Collier, C.D., Zhou, H.-C. Inorg. Chem. 2007, 46(9), 3432

${ }^{28}$ Hafizovic, J., Krivokapic, A., Szeto, K.C., Jakobsen, S., Lillerud, K.P., Olsbye, U., Tilset, M. Cryst. Grow. Des. 2007, 7(11), 2302

${ }^{29}$ Koh, K., Wong-Foy, A.G., Matzger, A.J., Angew Chem. Int. Ed. 2008; 47, 677.

${ }^{30}$ Koh, K., Wong-Foy, A.G., Matzger, A.J., J. Am. Chem. Soc. 2008; submitted for publication.

${ }^{31}$ Ma, S., Wang, X.S., Collier, C.D., Manis, E.S., Zhou, H.-C. Inorg. Chem. 2007, 46, 8499.

${ }^{32}$ Wang, B., Cote, A.P., Furukawa, H., O’Keefe, M., Yaghi, O.M. Nature 2008, 453, 207

${ }^{33}$ Humphrey, S.M., Chang, J.-S., Jhung, S.H., Yoon, J.W., Wood, P.T. Angew. Chem. Int. Ed. 2007, 46, 272.

${ }^{34}$ Wong-Foy, A.G.; Lebel, O.; Matzger, A.J. J. Am. Chem. Soc. 2007, 129, 15740-15741.

${ }^{35}$ Caskey, S.R.; Wong-Foy, A.G.; Matzger, A.J. J. Am. Chem. Soc. 2008, 130, 10870-10871.

${ }^{36}$ Volkringer, C., Meddouri, M., Loiseau, T., Guillou, N., Marrot, J., Ferey, G., Haouas, M., Taulelle, F., Audebrand, N., Latroche, M. Inorg. Chem. 2008, 47, 11892-11901.

${ }^{37}$ Cavka, J.H., Jakobsen, S., Olsbye, U., Guillou, N., Lamberti, C., Bordiga, S., Lillerud, K.P. J. Am. Chem. Soc. 2008, 130, 13850-13851.

${ }^{38}$ Dan-Hardi, M., Serre, C., Frot, T., Rozes, L., Maurin,G., Sanchez, C., Ferey, G. J. Am. Chem. Soc. 2009, 131, 10857-10859.

${ }^{39}$ Sonnauer, A., Hoffmann, F., Froba, M., Kienle, L., Duppel, V., Thommes, M., Serre, C., Ferey, G., Stock, N. Angew. Chem. Int. Ed. 2009, 48, 3791-3794.

${ }^{40}$ Khan, N.A., Jun, J.W., Jhung, S.H. Eur. J. Inorg. Chem. 2010, 1043-1048.

${ }^{41}$ (a) Koh, K., Wong-Foy, A.G., Matzger, A.J. Chem Comm 2009, 41, 6162-6164. (b) Koh, K., Wong-Foy, A.G., Matzger, A.J. J. Am. Chem. Soc. 2009, 131(12), 4184-4185. (c) Angew. Chem., Int. Ed. 2008, 47(4), 677-680.

${ }^{42}$ Dietzel, P.D.C., Besikiotis, V., Blom, R. J. Mater. Chem. 2009, 19(39), 7362-7370. 
${ }^{43}$ Yazaydin, A.O.; Snurr, R.Q.; Park, T.H.; Koh, K.; Liu, J.; LeVan, M. D.; Benin, A. I.; Jakubczak, P., Lanuza, M., Galloway, D.B., Low, J. J., Willis, R. R. J. Am. Chem. Soc. 2009, 131, 18198-18199.

${ }^{44}$ Potoff, J.J., Siepmann, J.I. AIChE J. 2001, 47, 1676.

${ }^{45}$ Mayo, S.L., Olafson, B.D., Goddard III, W.A. J. Phys. Chem. 1990, 94, 8897.

${ }^{46}$ Düren, T., Sarkisov, L., Yaghi, O.M., Snurr, R.Q. Langmuir 2004, 20, 2683.

${ }^{47}$ Frost, H., Düren, T., Snurr, R.Q. J. Phys. Chem. B 2006, 110, 9565.

${ }^{48}$ Walton, K.S., Millward, A.R., Dubbeldam, D., Frost, H., Low, J.J., Yaghi, O.M., Snurr, R.Q. J. Am. Chem. Soc. 2008, 130, 406.

${ }^{49}$ Dickey, A.N., Yazaydin, A.Ö., Willis, R.R., Snurr, R.Q., in preparation.

${ }^{50}$ (a) Wang, Y.; LeVan, M. D. Ind. Eng. Chem. Res. 2007, 46, 2141-2154. (b) Wang, Y.; LeVan, M. D. Ind. Eng. Chem. Res. 2008, 47, 3121-3128.

${ }^{51}$ LeVan, M. D.; Carta, G. In Perry's Chemical Engineers' Handbook, 8th ed.; Green, D. W., Perry, R. H., Eds.; McGraw-Hill: New York, 2008; Sec. 16, pp 22-24.

52 Twigg, M. V. Catalyst Handbook; Wolfe Publishing Ltd: London, 1989.

53 (a) Yang, Q. Y.; Zhong, C. L.; Chen, J. F. J. Phys. Chem. C 2008, 112, 1562-1569. (b) Liu, D. H.; Zheng, C. C.; Yang, Q. H.; Zhong, C. L. J. Phys. Chem. C 2009, 113, 5004-5009. (c) Zhao, Z. X.; Li, Z.; Lin, Y. S. Ind. Eng. Chem. Res. 2009, 48, 10015-10020.

${ }^{54}$ Low, J.J., Benin, A.I., Jakubczak, P., Abrahamian, J.F., Willis, R.R. J. Am. Chem. Soc. 2009, 131, 15834-15842.

55 Yazaydin, A.Ö., Benin, A.I., Faheem, S.A., Jakubczak, P., Low, J.J., Willis, R.R., Snurr, R.Q. Chem. Mater. 2009, 21, 1425.

${ }^{56}$ Breck, D. W. Zeolite Molecular Sieves: Structure, Chemistry, and Use; Wiley: New York, 1973.

${ }^{57}$ Kondo, A.; Daimaru, T.; Noguchi, H.; Ohba, T.; Kaneko, K.; Kanoh, H. J. Colloid Interface Sci. 2007, $314,422-426$.

58 (a) Gu, J. Z.; Lu, W. G.; Jiang, L.; Zhou, H. C.; Lu, T. B. Inorg. Chem. 2007, 46, 5835-5837. (b) Chen, Y.; Kondo, A.; Noguchi, H.; Kajiro, H.; Urita, K.; Ohba, T.; Kaneko, K.; Kanoh, H. Langmuir 2009, 25, $4510-4513$.

${ }^{59}$ Wang, Q. M.; Sheng, D.; Bulow, M.; Lau, M. L.; Deng, S.; Fitch, F. R.; Lemcoff, N. O.; Semanscin, J. Microporous Mesoporous Mater. 2002, 55, 217-230.

${ }^{60}$ (a) Castillo, J. M.; Vlugt, T. J. H.; Calero, S. J. Phys. Chem. C 2008, 112, 15934-15939. (b) Kusgens, P.; Rose, M.; Senkovska, I.; Frode, H.; Henschel, A.; Siegle, S.; Kaskel, S. Microporous Mesoporous Mater. 2009, 120, 325-330.

${ }^{61}$ Lee, K. B.; Sircar, S. AIChE J. 2008, 54, 2293-2302.

${ }^{62}$ Wang, Y.; LeVan, M. D. J. Chem. Eng. Data 2009, 54, 2839-2844. 
63 Wu, H.; Zhou, W.; Yildrim, T. J. Am. Chem. Soc. 2009, 131, 4995-5000.

64 (a) Prestipino, C.; Regli, L.; Vitillo, J. G.; Bonino, F.; Damin, A.; Lamberti, C.; Zecchina, A.; Solari, P. L.; Kongshaug, K. O.; Bordiga, S. Chem. Mater. 2006, 18, 1337-1346. (b) Forster, P. M.; Eckert, J.;

Chang, J. S.; Park, S. E.; Ferey, G.; Cheetham, A. K. J. Am. Chem. Soc. 2003, 125, 1309-1312.

${ }^{65}$ Chowdhury, P.; Bikkina, C.; Meister, D.; Dreisbach, F.; Gumma, S. Microporous Mesoporous Mater. 2009, 117, 406-413.

${ }^{66}$ Millward, A. R.; Yaghi, O. M. J. Am. Chem. Soc. 2006, 127, 17998-17999.

${ }^{67}$ Liu, J.; Wang, Y.; Benin, A. I.; Jakubczak, P.; Willis, R. R.; LeVan, M. D. Langmuir 2010, 26, 1430114307. 


\section{APPENDIX 1}

\section{Peer Review (29 April 2009) Summary}

Overall the project received a rating of 4.58 out of 5. Three Recommendations and one Action Item were suggested by the panel. These are pasted below, along with team responses to each in Arial italic.

Recommendation 1: Focus on the stability of sorbents.

The project team should focus on sorbent stability under operating conditions and sorbent tolerance to contaminants. Sorbent exposure testing to contaminants should be conducted to understand the impact of contaminants on sorbent performance, life, and stability, as well as the MOFs' mechanisms of adsorption (chemical, physical, or both). The successful completion of this study should be made a project milestone.

Investigation of sorbent stability is part of the project scope and is currently in progress as part of Task 17. Specifically, the stability of formed products will be measured in the presence of $\mathrm{SO}_{x}$ and $\mathrm{NO}_{x}$ under typical flue gas conditions. Adsorption kinetics will also be measured under typical flue gas conditions. Finally, isotherm data will be collected for the optimized formed product at multiple temperatures and water levels to provide inputs for detailed process simulations.

Recommendation 2: Develop a process for more innovative water removal. UOP stated it is presently considering the use of a separate thermal swing adsorption (TSA) system to remove water prior to the use of a MOF-PSA system that will remove $\mathrm{CO}_{2}$; the addition of a stand-alone TSA for dehydration adds non-trivial capital and operating expenses, limiting the potential reduction in $\mathrm{COE}$ increases available due to UOP's novel $\mathrm{CO}_{2}$ removal technology. UOP needs to consider incorporating the water removal step within the MOF-PSA $\mathrm{CO}_{2}$ removal system. This is commonly done in hydrogen $\left(\mathrm{H}_{2}\right)$-PSA systems in steam reformer plants for $\mathrm{H}_{2}$ production that remove water first and then remove $\mathrm{CO}_{2}$ by using a dual adsorbent bed. The first layer of adsorbent, typically activated carbon, is formulated specifically for water removal; these adsorbents are commonly found in industry and can be supplied by UOP. Activated carbon could potentially reduce the overall cost of the adsorbents in the system, since it costs approximately $\$ 1 / 1 \mathrm{~b}$, while the MOFs are projected to cost more than $\$ 20 / \mathrm{lb}$. The second layer of adsorbent is the MOF, which is used for $\mathrm{CO}_{2}$ removal. Both layers are incorporated in the adsorber vessel in segregated layer form, and UOP is well aware of how to properly execute this process.

As part of Task 19 of the project, UOP will develop a detailed design of a process for removal of $\mathrm{CO}_{2}$ from flue gas using an optimized MOF, including pretreatment requirements and process conditions. It is currently anticipated that the design will include a stand-alone TSA system for water removal. Although the panel's recommendation to incorporate the water removal step within the MOF-VSA $\mathrm{CO}_{2}$ removal system is worthy of consideration, the current project does not include the time and UOP resources necessary to perform a detailed design and analysis of this process configuration. 
Recommendation 3: Evaluate the effects of adding binders on MOF performance. Early in the project, the project team should evaluate the effects that adding binders will have on the reduction of MOF performance.

This work was initially completed as part of Task 11 in Phase 2 and is nearly complete as part of Task 17 in Phase 3. Please note that we are actually quite late in the project and hence the "early in the project" does not apply at the current time.

Action Item: Determine a means toward achieving the DOE COE goal. UOP's economic projections of a 54\% increase in COE is a great improvement over baseline scenarios. However, it still is well above the DOE COE goal of a 35\% increase. The project team needs to develop a clear pathway to approaching or reaching the DOE cost goal by indicating what type of changes still need to be made to the MOFs and to the plant process.

[Original Response] As a part of either the final Economic Analysis Report or Final Technical Report, the team will estimate and/or predict what we would need to change/improve re: the MOFs and/or the proposed VSA process in order to more closely approach the target COE increase goal.

Final response. The team has concluded that it will take a combination of improved adsorbent (e.g., increased $\mathrm{CO}_{2}$ capacity and enhanced moisture stability), and improved process design (e.g., input stream pre-treatment, moisture control, pressure-drop reduction, optimized bed configuration, hybrid temperature and pressure cycles) in order to move significantly from a 65 to a $35 \% \mathrm{COE}$ increase. In a recent proposal submitted to ARPA-E (DE-FOA-0000208), we set forth plans to achieve these goals, as per:

A proposed work plan consisting of 11 Tasks distributed among three Project Elements (PEs), namely: (1) Process Model Development, (2) MOF Synthesis Development, and (3) Flue Gas Exposure Studies. In PE 1, we will revise and optimize our preliminary vPSA Process Model that was developed earlier in order to clarify the potential benefits of our CC process technology. Further, we will perform complex mixture molecular modeling studies in order to be able to predict how both typical and unusual (upsets) power plant operation will affect the MOF adsorbent. A more detailed look at specific contaminants at various levels will be examined as a separate Task. PE 1 will have an experimental component as well, where investigators will carry out fast-cycle and other relevant adsorbent screening evaluations in order to more fully understand how improvements in operation might further lower the $\mathrm{CC}$ contribution to the cost of electricity (COE).

PE 2 is focused on the scale-up of the best MOF CC adsorbent to date, Mg/DOBDC. The main focus of PE 2 will be on scaling up the synthesis of $\mathrm{Mg} / \mathrm{DOBDC}$ and of forming the resultant powder into macroscopic pellets suitable for commercial operation. In another aspect of the work, the synthesis of the organic linker used to hold the Mg-containing hubs together will be re-formulated in order to drive down its cost. This work will also be carried out with an eye toward potentially manufacturing the linker internally (at Honeywell) in order to help control raw material costs. A final aspect of PE 2 will be on 
considering the Next Generation MOF CC adsorbent. In most Development projects, we have found that it is critical to invest a modest level of resources into the Next Generation material and process improvements.

The third Project Element, PE 3, brings it all together. That is, the best process configurations suggested from PE 1, coupled with the scaled-up Mg/DOBDC in PE 2, will be combined and systematically optimized in simulated and real flue gas (FG) environments. PE 3 will be divided roughly into three year-long phases where immersion tests, simulated FG tests, and real FG tests, respectively, are carried out. Cyclic operation will also be a part of PE 3 . 


\title{
APPENDIX 2
}

\section{Carbon Dioxide Removal from Flue Gas Using Microporous Metal Organic Frameworks}

\author{
Process Design and Economics Analysis Report
}

David Wegerer, Mark Davis, David Greer, and Richard Willis*

* Principal Investigator

richard.willis@uop.com

UOP LLC, a Honeywell Company

25 E. Algonquin Road

Des Plaines, IL 60017-5016

Phone: (847) 391-3190; Fax: (847) 391-3719

Report Issued: August 2010

Contract Number: DE-FC26-07NT43092 


\section{Contents (APPENDIX 2)}

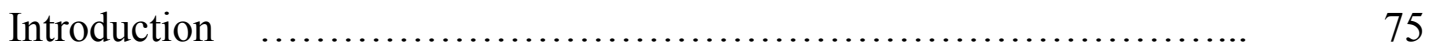

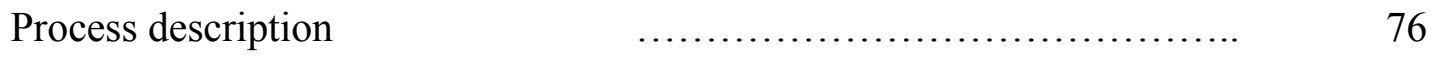

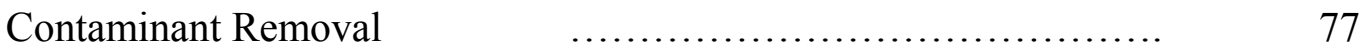

vPSA Feed Compressor and Cooler $\quad \ldots \ldots \ldots \ldots \ldots \ldots \ldots \ldots \ldots \ldots \ldots \ldots . \ldots \ldots$

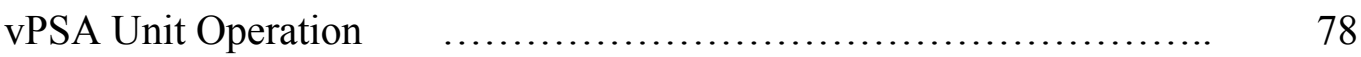

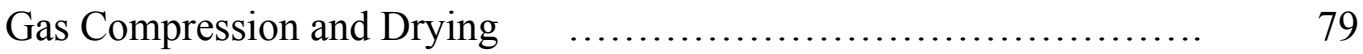

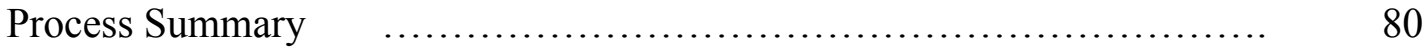

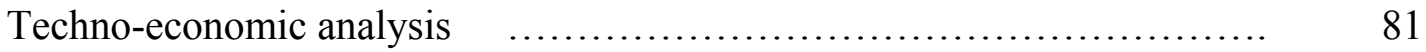

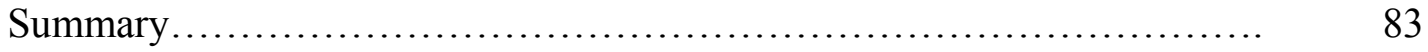

\section{Disclaimer}

"This report was prepared as an account of work sponsored by an agency of the United States Government. Neither the United States Government nor any agency thereof, nor any of their employees, makes any warranty, express or implied, or assumes any legal liability or responsibility for the accuracy, completeness, or usefulness of any information, apparatus, product, or process disclosed, or represents that its use would not infringe privately owned rights. Reference herein to any specific commercial product, process, or service by trade name, trademark, manufacturer, or otherwise does not necessarily constitute or imply its endorsement, recommendation, or favoring by the United States Government or any agency thereof. The views and opinions of authors expressed herein do not necessarily state or reflect those of the United States Government or any agency thereof." 


\section{Introduction}

This report summarizes UOP's preliminary assessment of a MOF-based vPSA (vacuumpressure swing adsorption) process for $\mathrm{CO}_{2}$ capture from coal-fired power plant flue gas. This Report reveals that this technology is equal to, or perhaps more technically feasible than the other current and emerging technologies for flue gas $\mathrm{CO}_{2}$ capture. For example, MOFs offer high capacity, high mass transfer rates, and ease of regeneration at typical flue gas temperature and pressure. Early indications suggest that $\mathrm{MOF} \mathrm{CO}_{2}$ selectivities over other flue gas components are reasonable, and that MOF contaminant stability may be manageable. Further, the UOP process will not require high quality steam from the main boilers for regeneration, which minimizes plant reconfiguration in a retrofit installation. Some details related to the UOP MOF-based vPSA process are provided in Table 1.

Table 1. Selected details on the MOF-based vPSA carbon dioxide capture process.

\begin{tabular}{|l|l|c|}
\hline & \multicolumn{1}{|c|}{ Parameter } & Expected Range \\
\hline \multirow{5}{*}{ Sorbent properties } & Type of sorbent & MOF \\
\cline { 2 - 3 } & Heat of adsorption $\left(\mathrm{kJ} / \mathrm{mole}^{\mathrm{CO}} \mathrm{C}_{2}\right.$ ) & $40-60$ \\
\cline { 2 - 3 } & $\mathrm{CO}_{2}$ loading/working capacity, wt.\% & $10-20$ \\
\cline { 2 - 3 } & Surface area, sq $\mathrm{m} / \mathrm{g}$ & $500-2000$ \\
\cline { 2 - 3 } & Packing density, $\mathrm{g} / \mathrm{cm}^{3}$ & $0.3-0.7$ \\
\cline { 2 - 3 } & Particle size $(\mathrm{mm})$ & $0.5-2.0$ \\
\cline { 2 - 3 } & Thermal stability, ${ }^{\circ} \mathrm{C}$ & $250-400$ \\
\cline { 2 - 3 } & Hydrothermal stability, ${ }^{\circ} \mathrm{C}$ & $100-200$ \\
\hline \multirow{5}{*}{ Process Configuration } & Cycle time (fixed bed), minutes & $4-16$ \\
\cline { 2 - 3 } & Pressure drop (fixed bed), psia & $1-2$ \\
\hline \multirow{5}{*}{ Operating conditions } & Adsorption temperature, ${ }^{\circ} \mathrm{C}$ & $25-45$ \\
\cline { 2 - 3 } & Adsorption pressure, atm. & $0.1-0.15$ \\
\cline { 2 - 3 } & $\mathrm{CO}_{2}$ capture efficiency, $\%$ & $90-95$ \\
\cline { 2 - 3 } & Regeneration method & vPSA \\
\cline { 2 - 3 } & Regeneration temperature, ${ }^{\circ} \mathrm{C}$ & $25-45$ \\
\cline { 2 - 3 } & Regeneration pressure, atm. & $0.01-0.05$ \\
\hline \multirow{2}{*}{ Product Quality } & $\mathrm{CO}_{2}$ purity, $\%$ & $90-98$ \\
\cline { 2 - 3 } & $\mathrm{N}^{2}$ concentration, $\%$ & Balance \\
\hline
\end{tabular}

This Report addresses two Subtasks and two Deliverables, as outlined here:

\section{Subtask 19.1 - Process Concept Development}

UOP will develop a process for $\mathrm{CO}_{2}$ removal from flue gas using the optimized MOF materials and process parameters identified in Phase 3, documented in a Process Design Report. The Process Design Report will include a detailed design of a process for removal of $\mathrm{CO}_{2}$ from flue gas using the optimized MOF, including pretreatment requirements and process conditions. 


\section{$\underline{\text { Subtask } 19.3 \text { - Economic Analyses }}$}

The economics of the proposed process will be analyzed, using the parameters developed in the material testing and process concept. The economic analysis will be performed in accordance with the guidelines set forth in NETL's Carbon Capture and Sequestration Systems Analysis Guidelines. The results will be documented in an Economic Analysis Report.

\section{$\underline{\text { Deliverables }}$}

Process Design Report, detailing the design of a process for removal of $\mathrm{CO}_{2}$ from flue gas using the optimized MOF, including pretreatment requirements and process conditions.

Economic Analysis Report, detailing the inputs, assumptions and results of estimating the costs and energy requirements for a commercial scale $\mathrm{CO}_{2}$ separation unit using the MOF-based adsorption process.

As suggested by our Program Manager at NETL, these two Deliverables will be combined herein into one report, a.k.a., a "Process Design and Economics Analysis Report." This Report is modeled after a similar Report on the Econamine MEA-based absorption process. ${ }^{1}$

\section{MOF-based vPSA Carbon Dioxide Capture Process Description}

A Carbon Dioxide Recovery (CDR) facility is used to remove 90 percent of the $\mathrm{CO}_{2}$ in the flue gas exiting the FGD unit, purify it, and compress it to a supercritical condition. The flue gas exiting the FGD unit contains about 1 percent more $\mathrm{CO}_{2}$ than the raw flue gas because of the $\mathrm{CO}_{2}$ liberated from the limestone in the FGD absorber vessel. The CDR facility is comprised of the flue gas supply, vPSA feed compression, VPSA feed cooler, the vPSA unit operation, vPSA evacuation compressor, and $\mathrm{CO}_{2}$ compression and drying. The vPSA process will be a proprietary design from UOP LLC, a Honeywell Company. The vPSA process will utilize a MOF adsorbent, specifically, $\mathrm{Mg} / \mathrm{DOBDC}$, to recover $\mathrm{CO}_{2}$ from the flue gas. This process is designed to recover high-purity $\mathrm{CO}_{2}$ from low-pressure streams that contain oxygen, such as flue gas from coal-fired power plants, gas turbine exhaust gas, and other waste gases. 


\section{Contaminant Removal}

It is anticipated that $\mathrm{CO}_{2}$ recovery will be integrated into a modern $\mathrm{PC}$ power plant with state-of-the-art contaminant removal trains in place (see Figure 1)

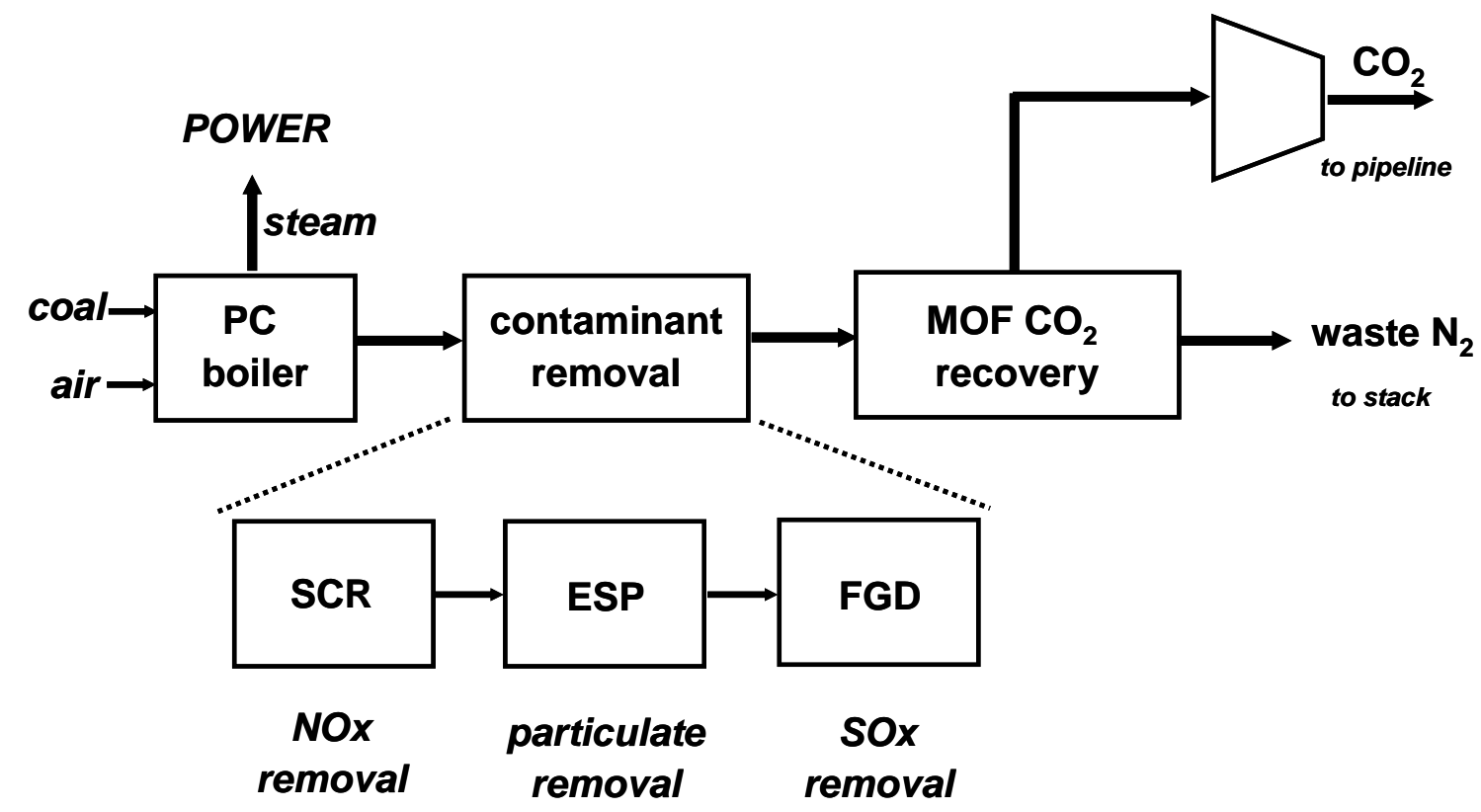

Figure 1. Integration of MOF-based vPSA $\mathrm{CO}_{2}$ capture process into a pulverized coal power plant.

This train will include a selective catalytic reduction (SCR) unit for removal of nitrogen oxides, an electro-spray collector unit for removal of particulates, and a flue gas desulfurization unit to remove oxides of sulfur. Additionally, the MOF based adsorption system could be augmented with existing adsorbent technology to provide even more complete removal of other contaminants such as $\mathrm{Hg}$ from the flue gas.

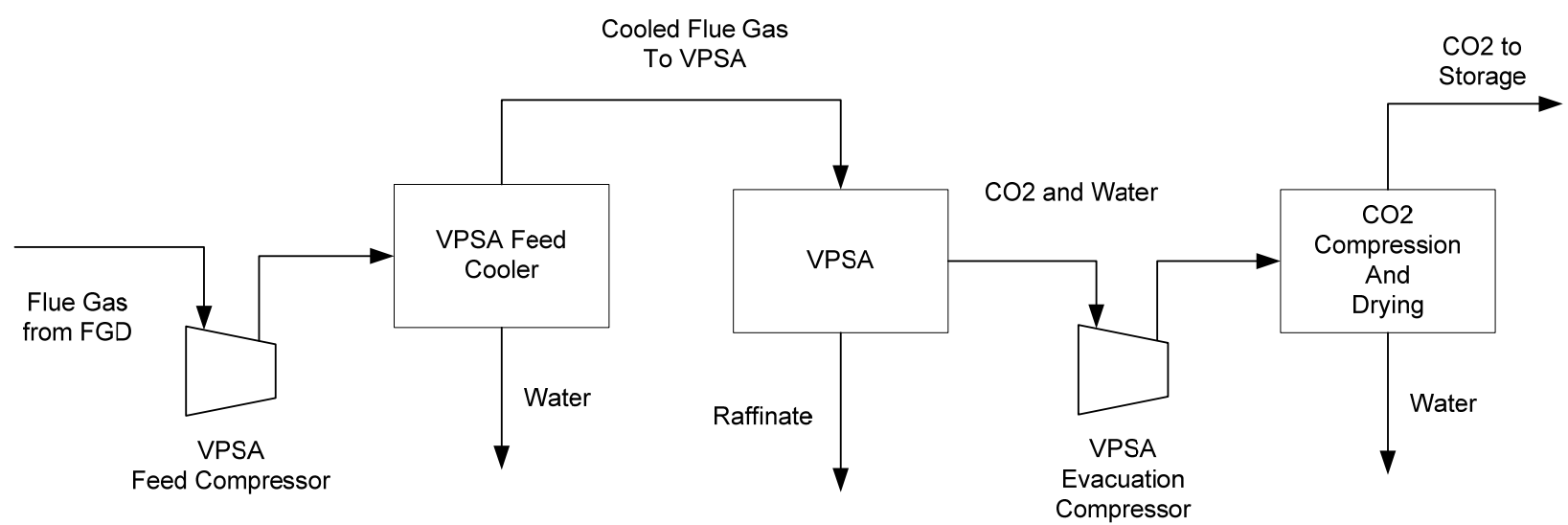

Figure 2. The MOF-Based $\mathrm{CO}_{2}$ capture system process schematic. 


\section{vPSA Feed Compressor and Cooler}

The MOF-based vPSA $\mathrm{CO}_{2}$ recovery system will be located after the Contaminant Removal section and before the final $\mathrm{CO}_{2}$ Compression and Drying section. The nitrogen-rich waste stream (raffinate) will be sent to the stack. A process schematic of the process is provided as Figure 2.

Downstream from the FGD and Polishing Scrubber, flue gas pressure is boosted in the vPSA Feed Compressor by approximately 4.8 psi to make up for pressure drop in the contaminant removal section and to maximize the adsorption of $\mathrm{CO}_{2}$ in the vPSA unit. The vPSA Feed Compressor will be followed by a vPSA Feed Cooler to lower the temperature of the flue gas to approximately $100{ }^{\circ} \mathrm{F}$. It is likely that two parallel operating vPSA Feed Compressor and vPSA Feed Cooler trains will be required. Compressing and cooling the flue gas to $100{ }^{\circ} \mathrm{F}$ will reduce the flue gas volume flow rate (ACFM) by up to $35 \%$ and concurrently knock out at least $75 \%$ of the water vapor originally present in the flue gas. The combined lower volume, lower temperature, and lower water content will allow the vPSA unit to operate more efficiently and effectively on the resultant flue gas stream.

Water management. Cooling water is provided from the PC plant circulating water system and returned to the PC plant cooling tower. The CDR facility requires cooling water for flue gas cooling, vPSA effluent cooling and $\mathrm{CO}_{2}$ compression inter-stage cooling. The cooling water requirement for the CDR facility is $\sim 100,000 \mathrm{gpm}$, assuming cooling water supply at $60{ }^{\circ} \mathrm{F}$ and return at $80{ }^{\circ} \mathrm{F}$.

\section{vPSA Unit Operation: Carbon Dioxide Adsorption}

The cooled flue gas enters the vPSA unit and will flow in a radial fashion through a short bed of adsorbent in either a vertical or horizontal configuration depending upon the particular power plant's requirements. The adsorbent beds will consist of alumina for moisture polishing, and MOF for $\mathrm{CO}_{2}$ removal. In the vPSA conceptual design, at the end of the adsorption step the vessel first vents $\mathrm{N}_{2}$ raffinate to the stack to reduce the bed pressure to atmospheric pressure and then pressure equalizes with another vessel that had just completed the vacuum regeneration step. This would reduce the bed pressure to 7-8 psia. These steps also reduce the $\mathrm{N}_{2}$ stored in the vessel voids and minimize the amount that would be co-produced with the $\mathrm{CO}_{2}$. The adsorbent bed would then be evacuated to 0.5 psia to produce the $\mathrm{CO}_{2}$. For the purposes of the calculations it was assumed that there was negligible co-adsorption of $\mathrm{N}_{2}$ on the $\mathrm{CO}_{2}$ loaded adsorbent and that the $\mathrm{N}_{2}$ was primarily stored in the voids of the adsorbent vessel. The target $\mathrm{CO}_{2}$ delta loadings were based on producing a $>90 \% \mathrm{CO}_{2}$ purity stream during the regeneration step. The parasitic load for regeneration of the adsorbent is the compression energy associated with evacuating the vessel to $0.5 \mathrm{psia}$ and compressing the contents to at least $8.8 \mathrm{psig}$. The contribution of the $\mathrm{N}_{2}$ stored in the voids is quite small compared to the compression energy associated with desorbing the $\mathrm{CO}_{2}$. A purge step was not used in this process since the primary concern is producing a high purity $\mathrm{CO}_{2}$ stream. 
Some process details are provided in Table 1. Slightly cooled flue gas will be dried in alumina beds prior to introduction to the MOF adsorbent. Temperatures as high as 130 ${ }^{\circ} \mathrm{F}$ will not negatively impact performance. The target $\mathrm{CO}_{2}$ purity in the process will be at least $95 \%$. It is expected that about 500,000 pounds of alumina and about 1 million pounds of MOF adsorbent distributed in 32 radial flow beds will be required for this carbon dioxide capture technology when retrofitted to a $550 \mathrm{MW}$ coal-fired power plant.

Heat management. There are no heating or cooling steps within the vPSA unit operation, which is typical of vPSA processes. Since this is still a conceptual design, however, there remain other types of processes to consider depending upon future pilot study and other experimental results. In a vPSA process the heat of adsorption is released and is stored in the bed by a sensible temperature rise. The heat is removed during the desorption step as the adsorbent bed cools. Approximately $90 \%$ of the $\mathrm{CO}_{2}$ in the feed gas is adsorbed onto the MOF adsorbent, and the rest leaves the vPSA section to the stack.

Waste management. Other than spent adsorbent, there is no waste generated in this process beyond that expected from MEA-based absorption technology. In fact, the MOFbased vPSA process should generate much less waste because the adsorbent itself is not hazardous waste, nor is it expected that the MOF will generate any hazardous waste products via degradation.

vPSA Evacuation Compressor. The vPSA Evacuation Compressor will be used to remove $\mathrm{CO}_{2}$ streams at $95-97 \%$ purity from the adsorbent beds. The water that comes along with the $\mathrm{CO}_{2}$ will be removed during the $\mathrm{CO}_{2}$ Compression and Drying stage of the process. In order to facilitate the Gas Compression and Drying section CAPEX estimate, the vPSA evacuation compressor discharge pressure is 8.8 psig. This is the same pressure that the Econamine process delivers captured $\mathrm{CO} 2$ to the Gas Compression and Drying section.

\section{Gas Compression and Drying System}

In the compression section, the $\mathrm{CO}_{2}$ is compressed to $15.3 \mathrm{MPa}(2,215$ psia) by a sixstage centrifugal compressor with inter-stage cooling to $100^{\circ} \mathrm{F}$. The discharge pressures of the stages were balanced to give reasonable power distribution and discharge temperatures across the various stages.

Compression and inter-cooling was simulated in order to estimate power and cooling loads, but the TEG dehydration was not simulated. Power consumption was estimated assuming an isentropic efficiency of $84 \%$. During compression to $15.3 \mathrm{MPa}(2,215 \mathrm{psia})$ in the multiple-stage, intercooled compressor, the $\mathrm{CO}_{2}$ stream is dehydrated to a dew point of $-40^{\circ} \mathrm{F}$ with triethylene glycol. The virtually moisture-free supercritical $\mathrm{CO}_{2}$ stream is delivered to the plant battery limit as sequestration ready. 


\section{Process summary}

Table 2 summarizes the similarities and differences between MEA-based absorption technology and MOF-based vPSA adsorption technology. In commercial operation, the MOF-based PSA system will have a lower regeneration duty than the MEA process because of higher relative $\mathrm{CO}_{2}$ loading on a mass basis and because vPSA is a physical rather than chemical process. In other words, the MOF process has a lower heat of adsorption (physical) than the heat of reaction (chemical) for the MEA process. This means that the $\mathrm{CO}_{2}$ is less tightly held by the MOF and hence is easier to remove. As such, no high quality steam from the coal-fired boilers will be required to regenerate the MOF adsorbent. By contrast, the MEA process will require high quality steam in order to get the amine to an elevated temperature to release the $\mathrm{CO}_{2}$.

Operating conditions for the two processes will be similar, although some flue gas cooling could make the MOF process more efficient overall. One additional operational advantage is that MOFs are not toxic and are not expected to generate any toxic degradation products over time.

Table 2. Comparison of MEA absorption and MOF vPSA adsorption processes.

\begin{tabular}{|c|c|c|}
\hline FAQ & $\begin{array}{l}\text { MEA chemical absorption } \\
\text { process technology }\end{array}$ & $\begin{array}{l}\text { MOF-based vPSA physical } \\
\text { adsorption process technology }\end{array}$ \\
\hline $\begin{array}{l}\text { Discuss the potential } \\
\text { improvement upon } \\
\text { the absorption cycle } \\
\text { regeneration duty } \\
\text { compared to } \\
\text { commercial systems. }\end{array}$ & $\begin{array}{l}\text { - Thermal amine } \\
\text { regeneration is energy- } \\
\text { intensive due to high heat of } \\
\text { reaction and relatively low } \\
\mathrm{CO}_{2} \text { loading on a mass } \\
\text { basis. } \\
\text { - High quality steam from } \\
\text { the boilers will be required } \\
\text { to regenerate the absorbent. }\end{array}$ & $\begin{array}{l}\text { - Lower regeneration duty due to } \\
\text { higher relative loadings on a mass } \\
\text { basis and lower heat of desorption } \\
\text { since the separation is based on } \\
\text { physical adsorption } \\
\text { - No high quality steam is required } \\
\text { for the MOF vPSA process. }\end{array}$ \\
\hline $\begin{array}{l}\text { What are the flue gas } \\
\text { conditions required } \\
\text { to maximize the } \\
\text { performance of the } \\
\text { proposed } \\
\text { technology? Will } \\
\text { these conditions } \\
\text { require changes to } \\
\text { the balance of plant? }\end{array}$ & $\begin{array}{l}\text { - MEA systems operate at } \\
\text { atmospheric pressure and } \\
\text { approximately } 120^{\circ} \mathrm{F} \text {. }\end{array}$ & $\begin{array}{l}\text { - The MOF adsorption system can } \\
\text { operate at similar conditions to the } \\
\text { MEA system, but is more efficient } \\
\text { if operated at lower temperature ( } \\
\left.40{ }^{\circ} \mathrm{F}\right) \\
\text { - Back heat integration may be } \\
\text { used to improve overall energy } \\
\text { efficiency. }\end{array}$ \\
\hline $\begin{array}{l}\text { Discuss any by- } \\
\text { product or waste } \\
\text { created by the } \\
\text { capture technology. }\end{array}$ & $\begin{array}{l}\text { Degradation must be treated } \\
\text { as hazardous waste, with } \\
\text { high disposal costs and } \\
\text { environmental impacts. }\end{array}$ & $\begin{array}{l}\text { MOFs do not break down in the } \\
\text { separation/regeneration process, so } \\
\text { no such degradation products are } \\
\text { expected. }\end{array}$ \\
\hline
\end{tabular}




\section{Techno-economic (TE) Analysis}

As has been described by NETL, qualifying technologies need to demonstrate at least $90 \% \mathrm{CO}_{2}$ capture, so this cost analysis is based upon that capture target. Other basic assumptions utilized in this analysis were taken from the NETL Fossil Energy Power Plant Desk Reference. It should be noted that TPC was adjusted from 2006 to $2010 \$$. $^{2}$ Basic assumptions are provided in Table 3. The most significant ones are that the plant is a $580 \mathrm{MW}$ gross power producer that operates $85 \%$ of the time, that electricity costs about 6 cents per kilowatt-hour, and that the total cost of such a plant without $\mathrm{CO}_{2}$ capture is $\$ 979 \mathrm{MM}$.

Table 3. Basic assumptions for the techno-economic analysis.

\begin{tabular}{|l|c|}
\hline Assumption & Data \\
\hline Plant availability & $85 \%$ \\
\hline Base Case Busbar cost of electricty (\$/KWh) & 0.0633 \\
\hline Base Case Gross Power (MW) & 580 \\
\hline Base Case Production Capacity (MW) & 550.2 \\
\hline $\begin{array}{l}\text { TPC for a Base Case Supercritical PC power plant w/o } \\
\text { CO2 capture; 2010 \$ (\$/KWe) }\end{array}$ & 1779 \\
\hline $\begin{array}{l}\text { Cost of a 550 MW Base Case Power Plant w/o CO2 } \\
\text { capture; } 2010 \$(\mathrm{MM} \$ \text { ) }\end{array}$ & \\
\hline & \\
Cost for transport, storage and monitoring (\$/short ton) & 979 \\
\hline
\end{tabular}

The assumptions in Table 3 lead to the analysis inputs displayed in Table 4. Again, the $\mathrm{CO}_{2}$ captured values assume $90 \%$ capture.

The next step was to use available team data to predict commercial performance parameters such as bed size and configuration, and optimal temperatures and pressures. In short, the analysis is based upon a MOF material able to achieve a working capacity of $10.5 \%$ (lbs $\mathrm{CO}_{2} / \mathrm{lbs}$ adsorbent) at $97^{\circ} \mathrm{F}$. The working capacity value is a reasonably conservative value because recent team results suggest working capacities of $15 \%$ or higher are possible. An additional assumption is a MOF adsorbent system cycle time of 8 minutes. The team has shown that the best MOFs for $\mathrm{CO}_{2}$ capture have fast kinetics, so this is a reasonably conservative assumption as well. It is upon these assumptions, and others as described above, that the calculations of energy cost penalty and capital are based. 
Table 4. Other inputs for the techno-economic analysis.

\begin{tabular}{|l|c|}
\hline Input & Data \\
\hline Base Case Total Annual Power Production (KWh/year) & $4,096,789,200$ \\
\hline Base Case Total Full Cost of production (\$) & $277,762,308$ \\
\hline CO2 emission w/o capture (ton/year) & $3,631,301$ \\
\hline CO2 emission w/o capture (ton/KWh) & 0.00089 \\
\hline CO2 short tons captured annually (ton/year) & $3,268,171$ \\
\hline Cost for Transport, Storage and Monitoring (\$) & $11,111,781$ \\
\hline CO2 avoided (ton/year) & $2,910,606$ \\
\hline
\end{tabular}

As can be seen in Table 5, the estimated increase in electricity cost for the successful application of the proposed MOF-based $\mathrm{CO}_{2}$ capture technology is $65 \%$. This cost is reasonable, approaching the DOE goal of less than $35 \%$ increase in electricity cost. This value is also somewhat lower than the predicted $81 \%$ increase in electricity cost of the NETL baseline MEA-based $\mathrm{CO}_{2}$ capture system.

Table 5. Calculation of energy increases due to MOF-based $\mathrm{CO}_{2}$ capture process.

\begin{tabular}{|l|c|}
\hline Parameter & Output \\
\hline Power Loss Due to CO2 capture + compression (\% of base capacity) & $20 \%$ \\
\hline CO2 capture case Production Capacity (MW) & $\mathbf{4 4 1}$ \\
\hline CO2 Capture Case Total Annual Power Production (KWh) & $3,283,710,151$ \\
\hline Additional full cost due to CO2 capture and compression (\$) & $78,575,940$ \\
\hline Capital Cost Increase \% because of CO2 capture & $\mathbf{3 6 \%}$ \\
\hline CO2 Capture Case Total Full Cost of production (\$) & $367,450,028$ \\
\hline CO2 Capture Case full cost of electricity (\$/KWh) & $\mathbf{0 . 1 1}$ \\
\hline Cost Increase per KWh (\%) & $\mathbf{6 5 \%}$ \\
\hline CO2 captured (ton/KWh) & 0.000995 \\
\hline Cost of CO2 avoided (\$/ton) & $\mathbf{5 7}$ \\
\hline
\end{tabular}

The modeling leads to similarly positive conclusions about the cost to operate a coal fired power plant that has been integrated with the proposed $\mathrm{CO}_{2}$ capture. The net cost of $\mathrm{CO}_{2}$ avoided is calculated to be about $\$ 57$ per ton of $\mathrm{CO}_{2}$ avoided, as shown in Table 5. This compares favorably to the $\$ 72$ per ton predicted for an amine-based system. The cost of 
$\mathrm{CO}_{2}$ avoided is based on $580 \mathrm{MW}$ gross power production and current year (i.e 2010) process plant cost. Note that net power production for the non-capture base case, MOFbased $\mathrm{CO}_{2}$ capture technology, and amine-based capture technology are not equal. We also estimated the capital costs associated with integrating this $\mathrm{CO}_{2}$ capture technology into the NETL base case power plant as shown in Figure 2. The Total Capital Requirement (TCR) is \$354MM (2010 \$) and is a summation of \$219.5MM for a vPSA system using a MOF adsorbent, $\$ 42.1 \mathrm{MM}$ for $\mathrm{CO}_{2}$ compression and drying, and about \$92.4MM in interest, MOF inventory, start-up and other miscellaneous costs.

Based upon this cost analysis, we strongly believe that the proposed $\mathrm{CO}_{2}$ capture technology can compete favorably with other proposed technologies at the performance targets developed by NETL. It is assumed that improvements in process design and configuration, heat integration, and adsorbent performance could lower these cost estimates significantly.

\section{Summary}

A MOF-based $\mathrm{CO} 2$ capture process is shown to be less energy intensive and hence less expensive to operate than an amine-based process. This is because in commercial operation, the MOF-based PSA system will have a lower regeneration duty than the MEA process because of higher relative $\mathrm{CO}_{2}$ loading on a mass basis and because vPSA is a physical rather than chemical process. As such, no high quality steam from the coal-fired boilers will be required to regenerate the MOF adsorbent. By contrast, the MEA process will require high quality steam in order to get the amine to an elevated temperature to release the $\mathrm{CO}_{2}$. This suggests that the MOF-based process will be easier to integrate into existing power plants because the current boilers and associated piping will not be effected directly by the retrofit. Overall operating conditions for the two processes will be similar, although some flue gas cooling could make the MOF process more efficient overall. One additional operational advantage is that MOFs are not toxic and are not expected to generate any toxic degradation products over time.

The estimated increase in electricity cost for the successful application of the proposed MOF-based $\mathrm{CO}_{2}$ capture technology is $65 \%$. This cost is reasonable, approaching the DOE goal of less than $35 \%$ increase in electricity cost. This value is also somewhat lower than the predicted $81 \%$ increase in electricity cost of the NETL baseline MEAbased $\mathrm{CO}_{2}$ capture system. The net cost of $\mathrm{CO}_{2}$ avoided is calculated to be about $\$ 57$ per ton of $\mathrm{CO}_{2}$ avoided. This compares favorably to the $\$ 72$ per ton predicted for an amine-based system.

\section{References (APPENDIX 2)}

${ }^{1}$ Cost and Performance Baseline for Fossil Energy Plants, DOE/NETL-2007/1281: Volume 1: Bituminous Coal and Natural Gas to Electricity, Final Report (Original Issue Date, May 2007), p 313 317.

${ }^{2}$ Fossil Energy Power Plant Desk Reference, DOE/NETL-2007/1282: May 2007 (Julianne M. Klara, Senior Analyst) 


\title{
APPENDIX 3
}

\section{Carbon Dioxide Removal from Flue Gas Using Microporous Metal Organic Frameworks}

\author{
Pilot Study Design Report
}

Ozgur Yazaydin, Allison Dickey, Maria-Chiara Ferrari, Randall Q. Snurr, Stefano

Brandani, and Richard Willis*

* Principal Investigator

richard.willis@uop.com

UOP LLC, a Honeywell Company

25 E. Algonquin Road

Des Plaines, IL 60017-5016

Phone: (847) 391-3190; Fax: (847) 391-3719

Report Issued: September 2010

Contract Number: DE-FC26-07NT43092

\section{Disclaimer}

"This report was prepared as an account of work sponsored by an agency of the United States Government. Neither the United States Government nor any agency thereof, nor any of their employees, makes any warranty, express or implied, or assumes any legal liability or responsibility for the accuracy, completeness, or usefulness of any information, apparatus, product, or process disclosed, or represents that its use would not infringe privately owned rights. Reference herein to any specific commercial product, process, or service by trade name, trademark, manufacturer, or otherwise does not necessarily constitute or imply its endorsement, recommendation, or favoring by the United States Government or any agency thereof. The views and opinions of authors expressed herein do not necessarily state or reflect those of the United States Government or any agency thereof." 


\section{Introduction}

Using a post-combustion capture technique to remove $\mathrm{CO}_{2}$ from flue gas can be expensive and challenging for a variety of reasons. For example, since flue gas exists near atmospheric pressure and the partial pressure of $\mathrm{CO}_{2}$ is only $\sim 0.1 \mathrm{bar}$, the volume and size of the capture system will likely be quite large [1]. The existence of SOx and NOx in flue gas may also be a problem in PCC systems because these compounds may poison or reduce the $\mathrm{CO}_{2}$ uptake capacity of the adsorbent. Finally, compressing captured $\mathrm{CO}_{2}$ may add a significant cost and energy load to the process; some literature articles have discussed compressing $\mathrm{CO}_{2}$ to pressures as high as 100 bar, which would incur very large energy costs [1].

As a Deliverable for this Project, a pilot study to demonstrate $\mathrm{CO}_{2}$ removal from the slipstream of an electric power-generation plant is reported here as a Pilot Study Design Report. This Pilot Study Design Report describes the design of a two-stage pilot study comprising: (1) a preliminary pilot study in stand-alone pilot plant; and (2) a field study operating on slipstream from an operational plant.

(1) Design of a Stand-Alone Pilot Plant (NU). As has been described in the main section of the Final Technical Status Report, the M/DOBDC series not only adsorb the largest amount of $\mathrm{CO}_{2}$, but these MOFs also have the largest $\mathrm{CO}_{2} / \mathrm{N}_{2}$ selectivities. The GCMC binary mixture and IAST calculations identify $\mathrm{Ni} / \mathrm{DOBDC}$ and $\mathrm{Mg} / \mathrm{DOBDC}$ as being the two MOFs having the highest $\mathrm{CO}_{2} / \mathrm{N}_{2}$ selectivities. Thus, both adsorbents look like promising candidates for $\mathrm{CO}_{2}$ capture. It is therefore interesting to examine how these candidates would perform using a post-combustion technology such as Pressure Swing Adsorption (PSA).

PSA is used to separate gas species by exploiting the selectivity that an adsorbent shows for the gas species of interest (here $\mathrm{CO}_{2}$ ). We have modeled a pilot-scale PSA unit using process simulation software with Ni/DOBDC as the adsorbent. This PSA unit is sized to contain $2 \mathrm{~kg}$ of Ni/DOBDC. This is the typical scale pilot unit utilized at UOP to demonstrate commercial PSA performance. The resulting PSA specifications that we started to optimize are the amount of $\mathrm{CO}_{2}$ captured, the purity of the product stream, and the energy consumption. Since we are interested in capturing $\mathrm{CO}_{2}$, which is the more strongly adsorbed component, we will use a modified-form of PSA that is known as PSA-SVR (Solvent Vapor Recovery). The objective of PSA-SVR is to remove the diluting carrier gas (often referred to as the light component stream, here $\mathrm{N}_{2}$ ) and to collect the heavy component product (the "solvent vapor"). The modeling methodology of PSA-SVR is very similar to that of conventional PSA.

The PSA unit - actually referred to as vPSA henceforth owing to vacumm being pulled to lower pressue - will contain two beds and each bed will undergo four steps during one cycle, as shown in Figure 1: (1) feed pressurization, (2) high-pressure feed, (3) countercurrent blowdown, and (4) countercurrent purge with product. 


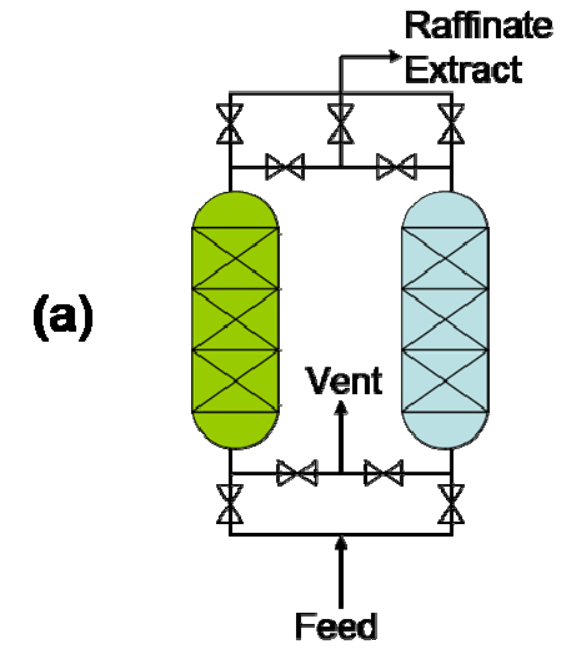

(b)

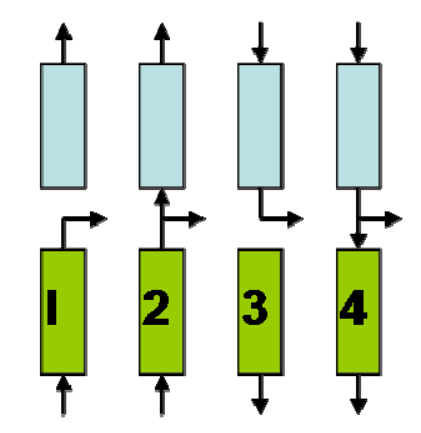

Figure 1: Diagram of a two-bed vPSA process (a) and the four steps that occur during in each vPSA cycle (b).

The raffinate stream consists of the less strongly adsorbed species (here $\mathrm{N}_{2}$ ) and is removed during the adsorption step at the feed pressure (step 2 in Figure 1). The extract stream consists of the more strongly adsorbed species (here $\mathrm{CO}_{2}$ ) and is recovered in the low-pressure (blowdown) purge stream that is used to regenerate the bed (step 4 in Figure 1). Thus, the purge and pressurization gases used in steps 1 and 4 come from the other bed as the light product of step 2. To maximize the amount of $\mathrm{CO}_{2}$ that is captured and the purity of the product stream, while minimizing the energy consumption, we tested a range of purge-to-feed ratios as well as altering the length of each of the four cycle steps to find the optimal operation conditions.

The vPSA process was simulated using the Aspen ADSIM Software with the objective of predicting the extent of the purity of the captured $\mathrm{CO}_{2}$. For the simulation this reduced to a simple two-bed process including three steps; feed pressurization (1), co-current depressurization (2), and vacuum. In each bed $2 \mathrm{kgs}$ of $\mathrm{Mg} / \mathrm{DOBDC}$ was used as the adsorbent material. Feed temperature and pressure was set to be $310 \mathrm{~K}$ and $1.3 \mathrm{~atm}$, respectively. Composition of the flue gas feed was $16 \% \mathrm{CO}_{2}$, and the balance was $\mathrm{N}_{2}$. A vacuum pressure of 0.05 atm was applied to adsorb the $\mathrm{CO}_{2}$ molecules in the bed. A flowsheet and the illustration of the steps of the vPSA process are shown in Figure 2.

These simulations yielded a "puzzling" product purity of $30 \%$, which is substantially lower than the expected outcome. There could be a few reasons for this. The simulations of the vPSA process faced challenges as most of the parameters, such as mass $\&$ heat transfer coefficients, particle porosity etc., were not experimentally available and had to be assumed based on parameters from similar materials. Besides, due to time restrictions, optimization of the vPSA process (such as length of each step, feed and vacuum conditions) were not explored. With the availability of parameters for $\mathrm{Mg} / \mathrm{DOBDC}$, and further process optimization it is likely that a higher purity will be obtained in the product stream. Moreover, the vPSA process could also benefit from the addition of new steps known to improve adsorption processes, such as pressure equilibration between beds. 


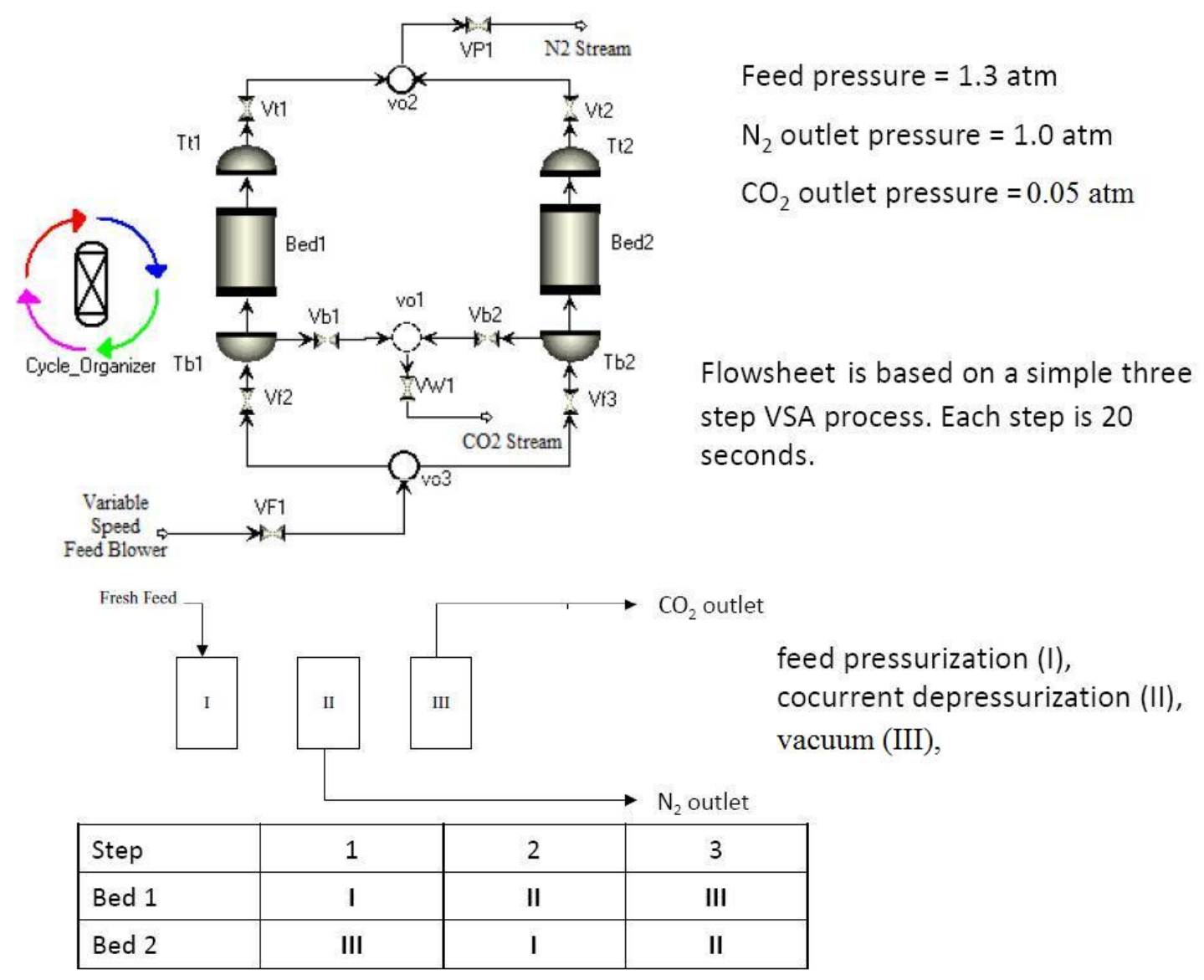

Figure 2. Flowsheet and illustration of process steps in vPSA.

Additional details related to the NU ADSIM model are provided as Supplementary Information at the end of this Report.

ADSIM simulations: Round 2 (UE). In parallel to the experimental work and simulation work at NU, researchers at UE worked on testing the ADSIM software for vPSA simulations based on literature data for $13 \mathrm{X}$ zeolite. UE also implemented simulations based on the data sent by NU.

UE started with implementing a simple breakthrough curve simulator under isothermal conditions, in order to compare the predictions with other UE simulation tools. This direct comparison led UE to identify a number of errors in ADSIM's mass balance equations and boundary conditions, which result in small systematic deviations. An example is shown in Figure 3. UE also confirmed that a Langmuir-Freundlich isotherm on an initially empty bed will lead to a numerical instability and the simulation will crash. Following this learning experience, UE implemented isothermal vPSA cycles and after a series of iterations they finally arrived at a working cycle for the direct multi-bed simulation and the direct single-bed simulation, but did not get the direct cyclic steady state simulator to run properly. 


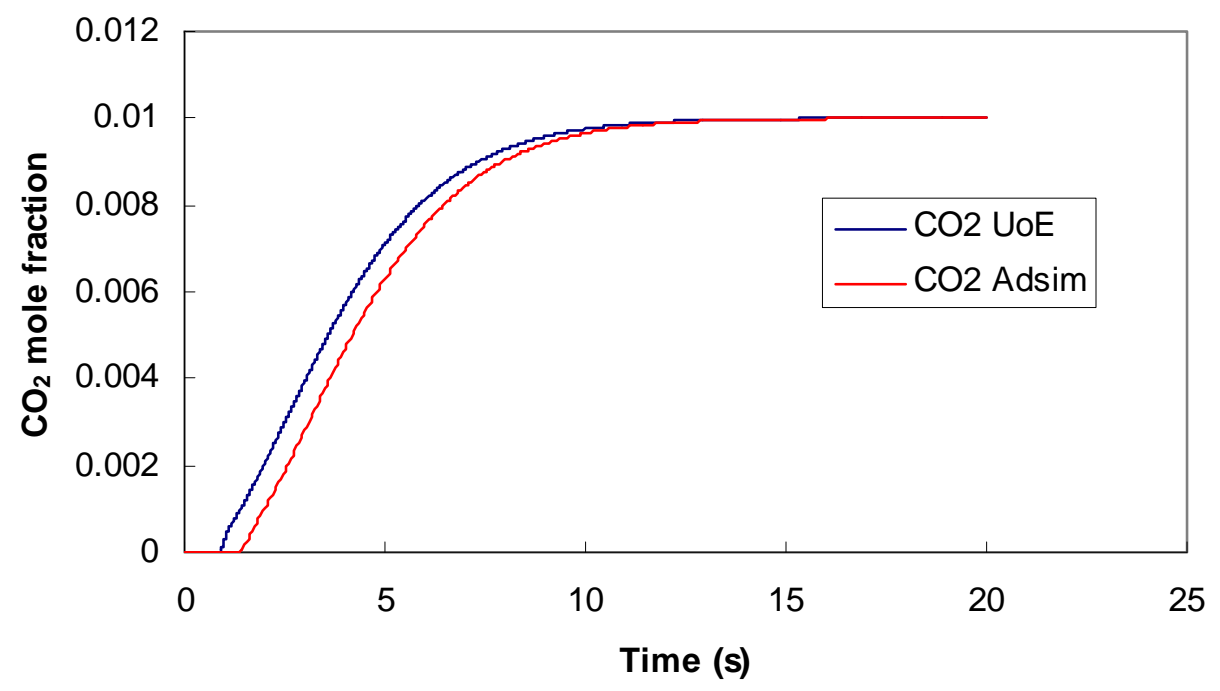

Figure 3. Comparison of breakthrough curves (short column, linear isotherm).

Rather than try and resolve this last case, UE decided to simulate the non-isothermal vPSA cycle. In this case they have determined that the energy balance equation has incorrect or missing terms, which may lead to stability issues if the default parameters are not modified. After significant effort and several iterations, they now have a cycle that runs for the multi-bed case, but it is difficult to evaluate the accuracy of these simulations. Note that UE is currently developing a 'cycle simulator.'

A summary of general conclusions on the ADSIM simulator follow:

1) Mass and energy balance equations are not accurate.

2) In dynamic open systems, the errors on the mass balance of the columns are going to be small, but depending on the model parameters errors resulting from the incorrect energy balance equations may be large.

3) The overall mass balance of the system is incorrect. Purities and recoveries on the exit streams do not add-up to the feed over the cycle. Concentrations are probably close to being accurate, but recoveries calculated by ADSIM can be $>100 \%$ ! See June presentation.

4) The graphic user interface to set up a cycle is relatively simple to use.

5) Default values in ADSIM should NOT be used for any parameter.

6) The numerical implementation is not very good and requires dispersion to be added in order to achieve stability.

7) The help and documentation often do not correspond to the options in the software (i.e. isotherms that can be selected are not all in the manual).

UE has not carried out a systematic study of the performance of a two bed vPSA cycle using ADSIM, but they have a simulation that now produces numerical output that can be used to determine the maximum purity of $\mathrm{CO}_{2}$ that could be achieved from a simple process (recall that recoveries as calculated by ADSIM are not reliable). 
(2) Design of a Field Study. A vPSA unit with Mg/DOBDC would be used in a pilot study to adsorb $\mathrm{CO}_{2}$ from a slipstream taken from an operating power plant. This study would use real flue gas rather than simulated flue gas [2] to adjust bed cycle times, examine how environmental conditions impact the $\mathrm{CO}_{2}$ uptake capacity of the adsorbent, and validate the vPSA startup and shutdown procedures. The objective is to capture $90 \%$ of the $\mathrm{CO}_{2}$ from a gas stream that contains at least $10 \% \mathrm{CO}_{2}$ by volume at no more than a $35 \%$ increase in the cost of energy. In addition, the purity of the product stream often needs to be $95 \% \mathrm{CO}_{2}$ for transport in a pipeline. Thus, we will minimize the amount of $\mathrm{N}_{2}, \mathrm{O}_{2}, \mathrm{H}_{2} \mathrm{O}$, and other impurities in the product stream.

In late 2009, We Energies, Alstom, and the Electric Power Research Institute (EPRI) completed a $5 \mathrm{MW}$ pilot study which examined $\mathrm{CO}_{2}$ capture from flue gas using an advanced chilled ammonia process at a coal-powered power plant in Pleasant Prairie, Wisconsin [3, 4]. In their study, the slipstream was $1 \%$ of the total exhaust gas and they captured more than $90 \%$ of the $\mathrm{CO}_{2}\left(\sim 40\right.$ tons of $\mathrm{CO}_{2}$ captured per day) from the flue gas. They were able to produce high-purity $\mathrm{CO}_{2}$ with low ammonia $(<10 \mathrm{ppm})$ and water content $(<2500 \mathrm{ppm})$ [3]. We suggest conducting the pilot study at the We Energies Pleasant Prairie Power Plant or a similar location because the plant was recently retrofitted with a selective catalytic reduction (SCR) system to reduce NOx emissions and a wet flue gas desulfurization (FGD) system to reduce $\mathrm{SO}_{2}$ emissions [3]. Studies could be performed with both wet and dry flue gas by cooling the slipstream for the "dry" studies to remove moisture and other condensable contaminants. Since the plant is located in Wisconsin, the pilot study should be conducted for at least a year so that the vPSA process is operational for all four seasons, which will provide information regarding how $\mathrm{CO}_{2}$ uptake and the operation of the vPSA system are affected by variations in the environmental conditions. Since this is a fairly lengthy study, we would simultaneously evaluate time-dependent alterations in adsorbent properties including the $\mathrm{CO}_{2}$ uptake, regeneration capabilities, pollutant contamination, and adsorbent attrition. Des Dillon, EPRI manager of the carbon capture retrofit project, has found that retrofitting power plants for CCS needs to be well integrated with the existing steam, waste, heat, water, and electricity resources that already exist at each power plant [5]. One advantage of conducting this pilot study at Pleasant Prairie would be that the results from the $\mathrm{Mg} / \mathrm{DOBDC} / \mathrm{vPSA}$ pilot study could be compared directly with those of the advanced chilled ammonia process without needing to incorporate power plant operational differences.

During vPSA operation, adsorption, depressurization, re-pressurization, and purge steps take place at varying times in different beds. One goal of this pilot study is to optimize the cycle times based on the local conditions, as well as to test the start-up and shutdown procedures. The cycle time schedule can become quite complex for a vPSA system with multiple beds and this schedule can be estimated using process modeling algorithms. In our proposed cycle, the flue gas enters the vPSA process at a temperature of $100^{\circ} \mathrm{F}$ and a pressure of 14.7 psia. The exiting flue gas pressure is sub-atmospheric since a low exit pressure increases the $\mathrm{CO}_{2}$ desorption from the adsorbent. Cycle times that were optimized using the computational model may need to be tuned for conditions that exist 
at the Pleasant Prairie power plant and for deficiencies of the process model. One advantage of using vPSA is that steam is not required to regenerate the adsorbent, thus the steam generator output is not decreased during $\mathrm{CO}_{2}$ capture [1]. A significant amount of energy will be required to compress the $\mathrm{CO}_{2}$ stream from sub-atmospheric to pipeline pressures. The pilot study may provide information that is useful in optimizing this pressurization step, such as the expected composition and pressure of the vPSA outlet.

\section{References (APPENDIX 3)}

[1 ] Assessment of Post-Combustion Capture Technology Developments. EPRI, Palo Alto, CA: 2007, 1012796.

[2] Strategic Analysis of the Global Status of Carbon Capture and Storage Report 1: Status of Carbon Capture and Storage Projects Globally Final Report. Global CCS Institute, Australia: 2009.

[3] Pleasant Prairie Carbon Capture Demonstration Project (Progress Report) . We Energies, Alstom, EPRI, Pleasant Prairie, Wisconsin: 2009.

[4] The Challenge of Carbon Capture. EPRI Journal, Palo Alto, CA: Spring, 2007.

[5] Retrofitting CO2 Capture. EPRI Journal, Palo Alto, CA: Summer, 2009. 


\section{Supplementary information (APPENDIX 3)}

A vPSA process was simulated using the Aspen ADSIM Software. $\mathrm{CO}_{2}$ concentration was found to be $30 \%$ in the product stream. The following sections give the details of the simulation.

\section{Design parameters}

Feed temperature $=310 \mathrm{~K}$

Feed pressure $=1.3 \mathrm{~atm}$

Vacuum pressure $=0.05 \mathrm{~atm}$
Ambient temperature $=298.15 \mathrm{~K}$

$\mathrm{CO}_{2}$ feed mol faction $=0.16\left(\right.$ balance $\left.\mathrm{N}_{2}\right)$

Waste pressure $=1.0 \mathrm{~atm}$

Adsorbent amount (Mads) $=2 \mathrm{~kg} \mathrm{Mg} \backslash \mathrm{DOBDC}$

Particle density $($ Rhop) $=909$ kg/m3 (from J. AM. CHEM. SOC. 2009, 131, 4995-5000)

Particle porosity - Intraparticle voidage $(E p)=0.7$ (assumed)

Void fraction - Interparticle voidage $(E \mathrm{E})=0.4$ (assumed)

Particle radius $(R p)=0.57 \mathrm{~mm}$ (based on NilDOBDC pellets)

$\mathrm{CO}_{2}$ mass transfer coefficient $=6.71 / \mathrm{s}$ (based on MTC measured in NilDOBDC by Vanderbilt)

$\mathrm{N}_{2}$ mass transfer coefficient $=0.67 \mathrm{1} / \mathrm{s}$ (estimated based on relative molecular weights of $\mathrm{N}_{2}$ and $\mathrm{CO}_{2}$, and their isotherms in $\mathrm{Mg} / \mathrm{DOBDC}$ )

Average $\mathrm{CO}_{2}$ heat of adsorption $=-41.7 \mathrm{~kJ} / \mathrm{mol}$ (measured by Vanderbilt)

Average $N_{2}$ heat of adsorption $=-13.4088 \mathrm{~kJ} / \mathrm{mol}$ (from simulations)

Specific heat capacity of $\mathrm{Mg} / \mathrm{DOBDC}=0.92 \mathrm{~kJ} / \mathrm{kg} / \mathrm{K}$ (Zeolite $13 \mathrm{X}$ heat capacity)

Heat transfer coefficients are taken from an $\mathrm{O}_{2}$ vPSA example provided within Adsim.

Volume of Bed (Vbed):

$$
\begin{aligned}
& \text { Vbed }=(\text { Mads } / \text { Rhop }) /(1-\mathrm{Ei})=\left(2 \mathrm{~kg} / 909 \mathrm{~kg} / \mathrm{m}^{3}\right) /(1-0.4)=0.003667 \mathrm{~m}^{3} \\
& \text { Set } \text { Dbed }=0.1 \mathrm{~m} \\
& \text { Vbed }=(\text { Dbed } / 2)^{\wedge} 2 * \pi * \text { Hbed } \\
& \text { Hbed } \left.=\text { Vbed } /[\text { Dbed } / 2)^{\wedge} 2 * \pi\right] \\
& \text { Hbed }=0.003667 \mathrm{~m}^{3} /\left[(0.1 / 2)^{\wedge} 2 * \pi\right] \\
& \text { Hbed }=0.46689 \mathrm{~m} \\
& \text { Hbed }=0.467 \mathrm{~m}
\end{aligned}
$$




\section{Fitting of Isotherms}

$\mathrm{CO}_{2}$ isotherms from experiments of Vanderbilt team and $\mathrm{N}_{2}$ isotherms from molecular simulations were used in Adsim after fitting them to equations of adsorption models.

Langmuir-Freundlich Eqn. used for fitting $\mathrm{CO}_{2}$ isotherms:
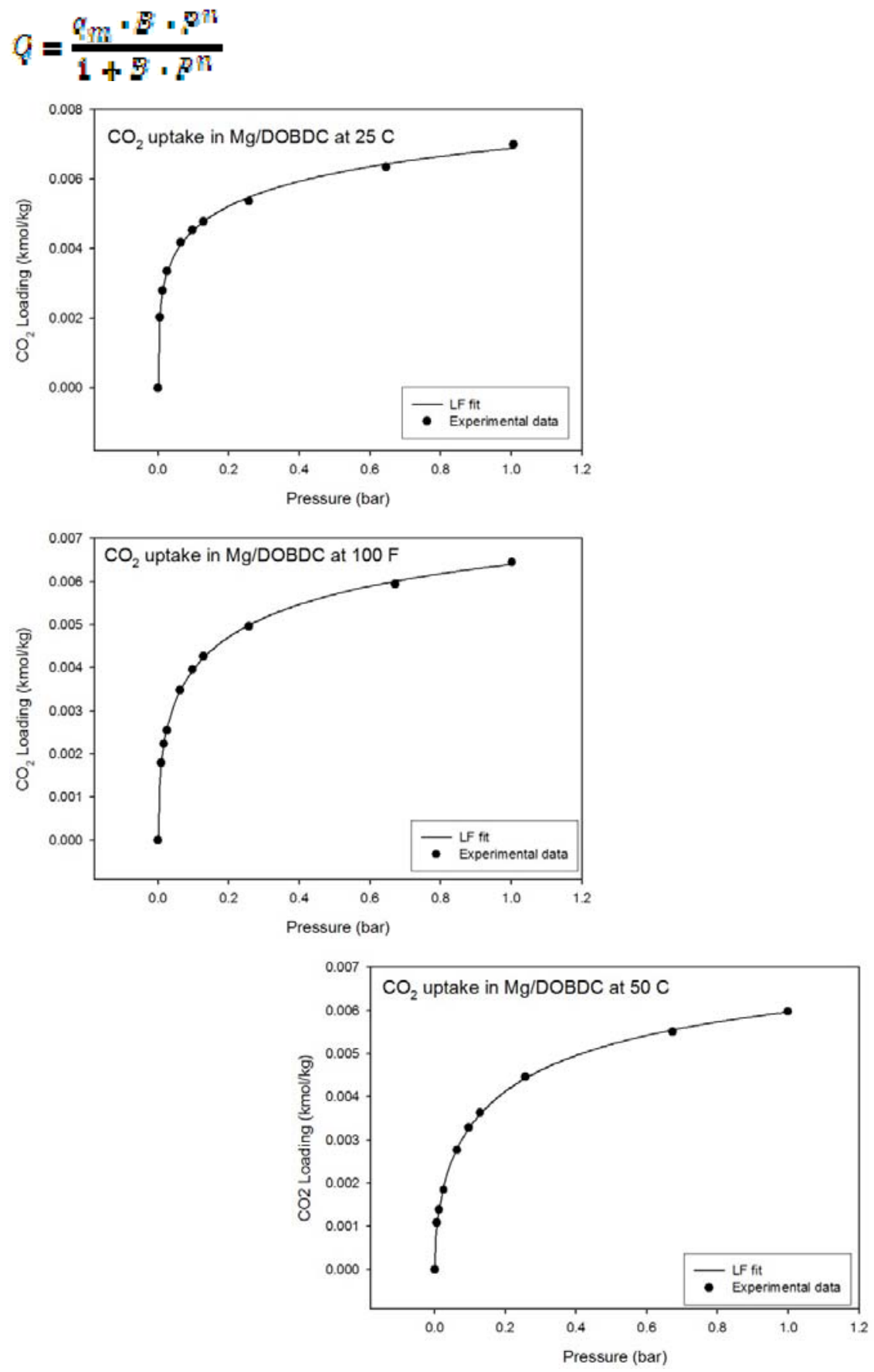


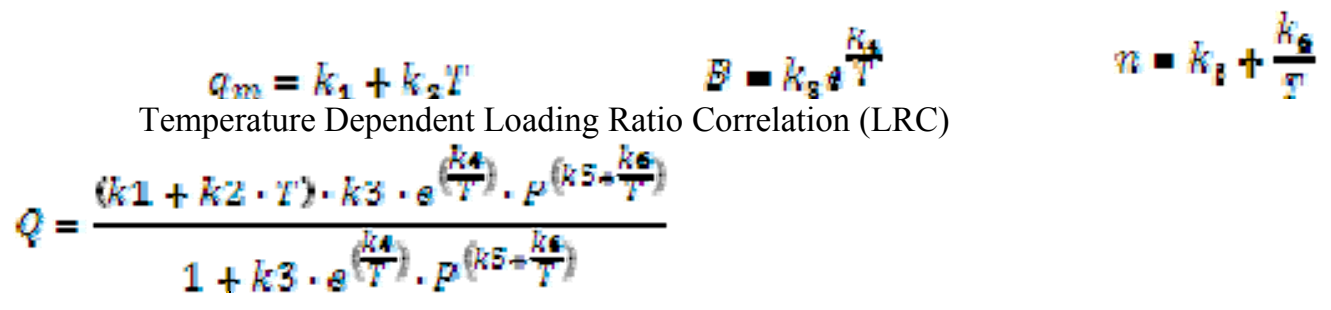
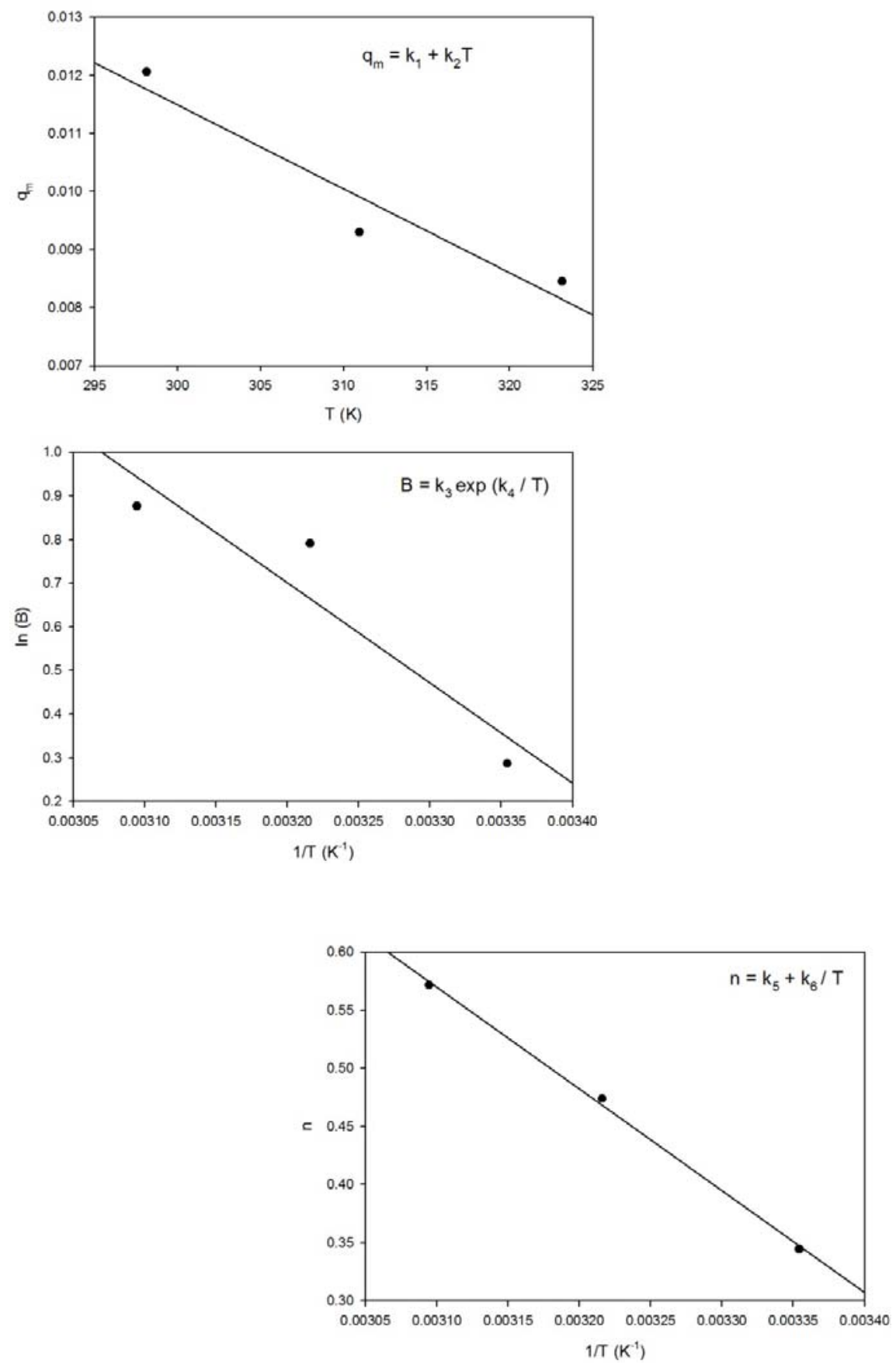


\begin{tabular}{|c|c|c|c|c|c|c|c|c|c|}
\hline & $\mathrm{q}_{\mathrm{m}}$ & B & $\mathrm{n}$ & $\mathrm{k} 1$ & $\mathrm{k} 2$ & $\mathrm{k} 3$ & $\mathrm{k} 4$ & $\mathrm{k} 5$ & k6 \\
\hline $25 \mathrm{C}$ & 0.0121 & 1.3325 & 0.3444 & \multirow{3}{*}{0.055} & \multirow{3}{*}{$-1.4488 \mathrm{e}-4$} & \multirow{3}{*}{3173.6435} & \multirow{3}{*}{-2300.5558} & \multirow{3}{*}{3.2896} & \multirow{3}{*}{-877.3241} \\
\hline $100 \mathrm{~F}$ & $9.3014 \mathrm{e}-3$ & 2.2062 & 0.4738 & & & & & & \\
\hline $50 \mathrm{C}$ & $8.4509 \mathrm{e}-3$ & 2.4009 & 0.5717 & & & & & & \\
\hline
\end{tabular}

Henrys' law eqn. use for fitting $\mathrm{N}_{2}$ isotherm (fitting graphs are not shown here):

$$
\begin{gathered}
Q=q_{m} \cdot P \\
q_{m}=k 1+k 2 \cdot T
\end{gathered}
$$

Temperature Dependent Loading Ratio Correlation (LRC):

$$
\begin{gathered}
Q=(k 1+k 2 \cdot T) \cdot P \\
k 1=1.7861 \cdot 10^{-5} \quad k 2=-4.8033 \cdot 10^{-6}
\end{gathered}
$$

\section{Flow Sheet}

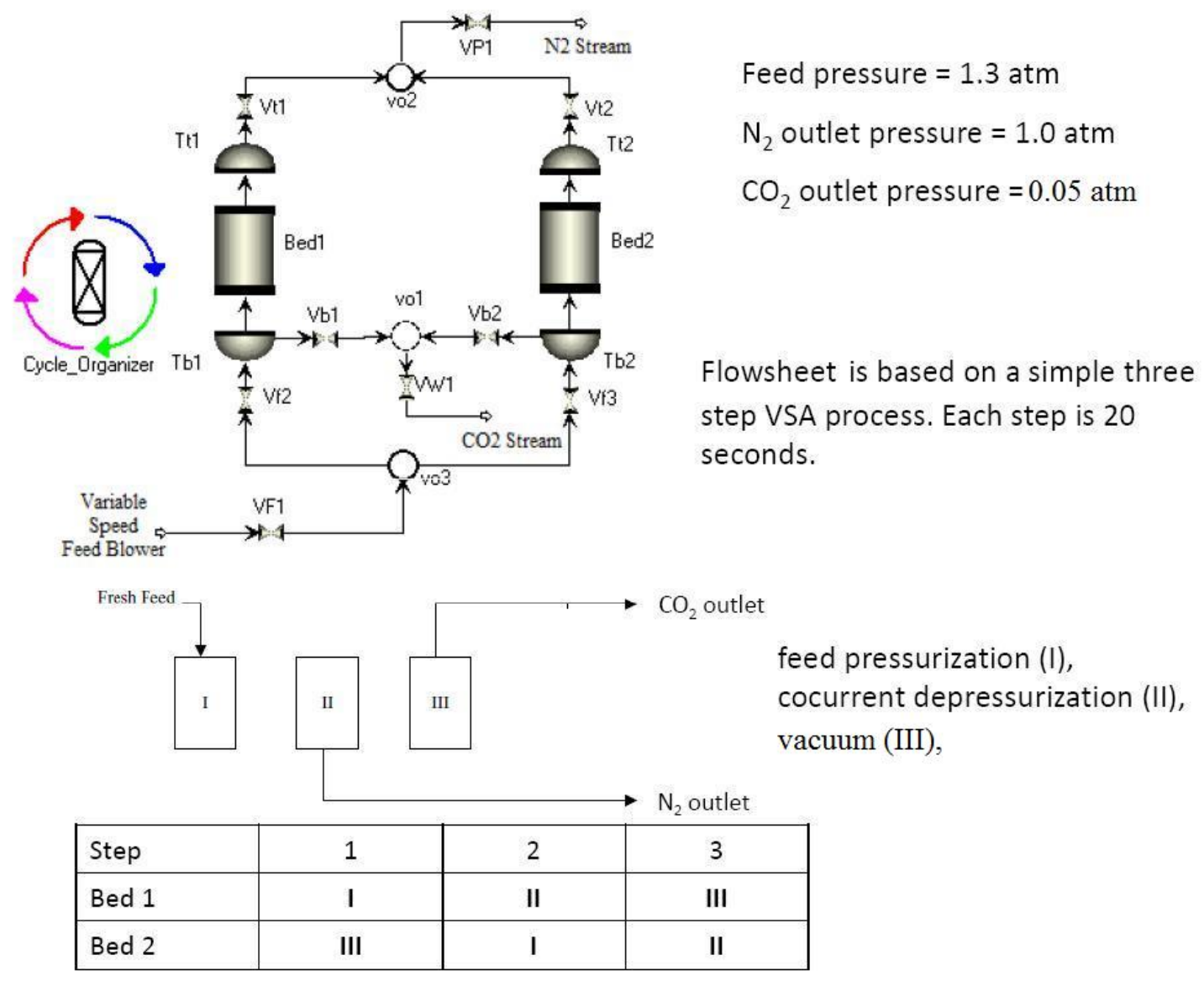




\begin{tabular}{|c|c|c|c|c|c|c|}
\hline & \multicolumn{3}{|c|}{ BED 1 } & \multicolumn{3}{c|}{ BED 2 } \\
\hline STEP 1 & \multicolumn{3}{|c|}{ Pressurize } & \multicolumn{3}{c|}{ Pacuum } \\
\hline STEP 2 & \multicolumn{2}{|c|}{ Co-current depressurization } & \multicolumn{3}{c|}{ Co-current depressurization } \\
\hline STEP 3 & \multicolumn{3}{|c|}{ Vacuum Valve Specs } & Vb2 & Vt2 \\
\hline & \multicolumn{3}{|c|}{ Vb1 } & Vt1 & Vf3 & Close \\
\hline & Vf2 & Close & Close & Close & Open & Close \\
\hline STEP 1 & Open & Close & Open & Open & Close & Open \\
\hline STEP 2 & Close & Open & Close & Close & Close & \\
\hline
\end{tabular}




\section{APPENDIX 4}

\section{Carbon Dioxide Removal from Flue Gas Using Microporous Metal Organic Frameworks}

Updated Commercialization Study (sanitized)

Ganesh Venimadhavan, Mark Davis, Peter Coughlin, David Steen, Gary Malott, Philip Jung, Kurt Vanden Bussche, David Lesch, David Penner, and Richard Willis*

* Principal Investigator

richard.willis@uop.com

UOP LLC, a Honeywell Company

25 E. Algonquin Road

Des Plaines, IL 60017-5016

Phone: (847) 391-3190; Fax: (847) 391-3719

Report Issued: September 2010

Contract Number: DE-FC26-07NT43092 


\section{Contents (APPENDIX 4)}

Introduction $\quad$..................................................... 98

Competitive overview and positioning ........................... 99

Entry barriers............................................... 104

Commercialization pathway................................... 105

Technology transfer alternatives................................ 108

Commercialization timeline..................................... 109

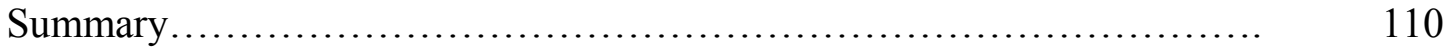

References ..................................................... $\quad 110$

\section{Disclaimer}

"This report was prepared as an account of work sponsored by an agency of the United States Government. Neither the United States Government nor any agency thereof, nor any of their employees, makes any warranty, express or implied, or assumes any legal liability or responsibility for the accuracy, completeness, or usefulness of any information, apparatus, product, or process disclosed, or represents that its use would not infringe privately owned rights. Reference herein to any specific commercial product, process, or service by trade name, trademark, manufacturer, or otherwise does not necessarily constitute or imply its endorsement, recommendation, or favoring by the United States Government or any agency thereof. The views and opinions of authors expressed herein do not necessarily state or reflect those of the United States Government or any agency thereof." 


\section{Introduction}

UOP has reviewed the preliminary commercialization study on a MOF-based process for adsorptive removal of carbon dioxide in flue gas from fossil-fuel fired power plants. This report represents an update of that Report based upon feedback from the NETL Project Manager. Those sections where significant changes were made will be highlighted.

The preliminary commercialization study, also known as Task 5.0 in the original Statement of Project Objectives (SOPO) consisted of the following sections:

Competitive overview and positioning. This section discussed the competitive dynamics in the industry and the current technology strategy to create a competitive advantage. A discussion and profile of key competitors of the proposed technology that indicates the advantages and disadvantages of the proposed technology to other competing technologies is included.

The techno-economic analysis has been updated and appears in a separate document "Process Design and Economic Analysis Report." This report can also be found as Appendix 2 of the project's Final Technical Status Report.

Entry barriers of the proposed technology.

A description of the commercialization pathway, potential customers, market potential, and a projected market share based on fundamental market analysis. Consideration of economic, complexity, and industry risk aversion shall be compared.

A discussion of various technology transfer alternatives.

A commercialization timeline with associated funding requirements, industry partnership requirements, and cost reduction projections at each stage of development. 


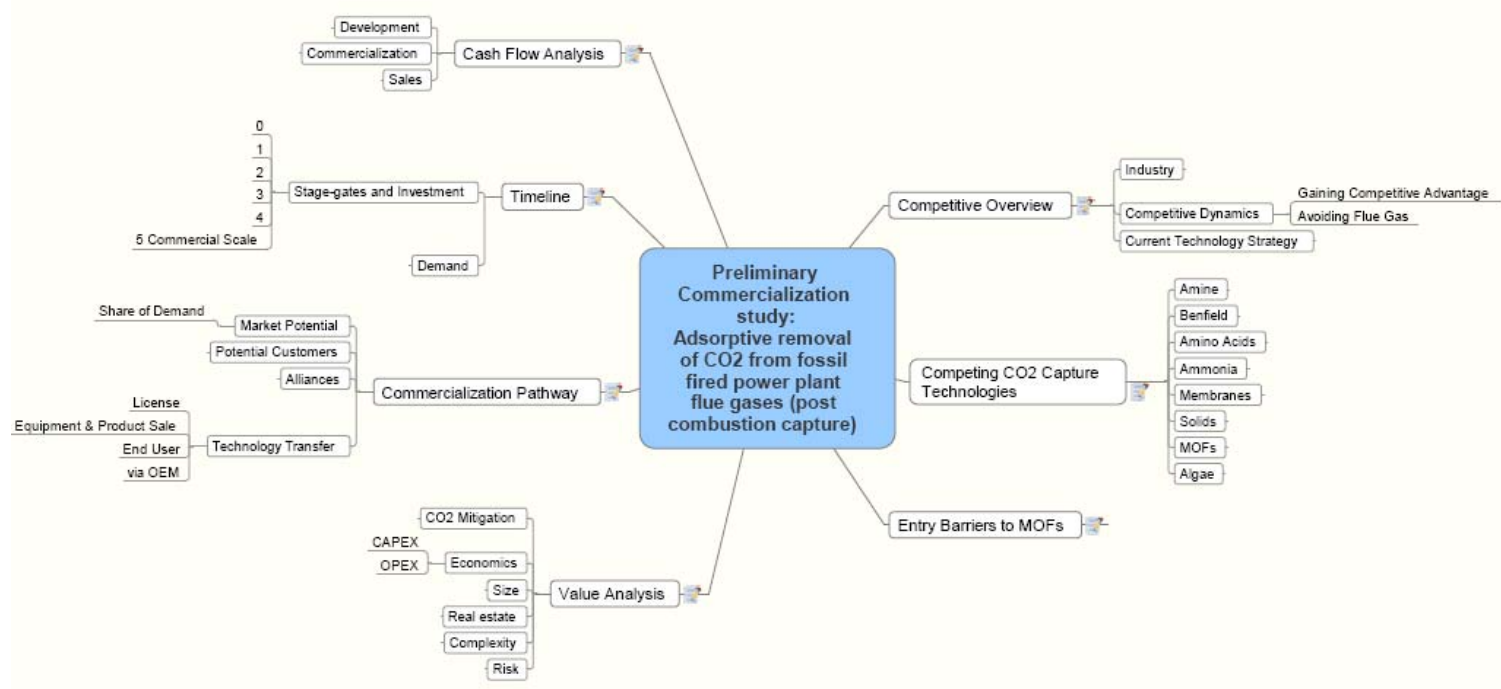

Figure 1. Thought map for this study.

\section{Competitive Overview and Positioning}

This section discusses the competitive dynamics in the industry and the current technology strategy to create a competitive advantage. A discussion and profile of key competitors of the proposed technology that indicates the advantages and disadvantages of the proposed technology to other competing technologies is included.

"Competing technologies" can be divided into a small number of current technologies and emerging technologies or technology enhancements currently under development. The state-of-the art in both areas is reviewed briefly here and compared to the proposed MOF-based $\mathrm{CO}_{2}$ capture process.

\section{Current Technologies}

Current technologies fall into two classes: solvent-based processes and processes using solid adsorbents. The current solvent based $\mathrm{CO}_{2}$ capture technology can be divided into three categories:

Reactive amine based processes

Reactive inorganic solvent based processes

Physical absorption processes

Examples of each are discussed below.

Reactive amine based processes. The reactive amine based process (which includes the UOP AmineGuard ${ }^{\mathrm{TM}}$ process) is the oldest technology available and has been in commercial applications for over 40 years. There has been an evolution of the amine 
solvents over time starting with primary amines such as monoethanolamine (MEA), to secondary amines such as diethanolamine (DEA) and more recently tertiary amines such as methyldiethanolamine (MDEA), and the MDEA based UCARSOL family of proprietary solvents from Dow Chemicals that are used in the AmineGuard ${ }^{\mathrm{TM}}$ process.

Amine-based systems are suitable for dilute $\mathrm{CO}_{2}$ streams, such as flue gas, which typically contains $10-18 \% \mathrm{CO}_{2}$ by volume. An amine scrubber system typically consists of an absorber where the solvent removes $\mathrm{CO}_{2}$ from the flue gas and a regenerator unit or stripper where the adsorbed $\mathrm{CO}_{2}$ is stripped from the solvent with steam. The flue gas is typically cooled before absorption with the amine solvent. A major drawback of amine systems is the heat is required to regenerate the solvent, which significantly reduces net efficiency. In addition, MEA solvents react with acid gases such as $\mathrm{SO}_{2}$ and $\mathrm{NO}_{2}$ to form heat stable salts that reduce the capacity of the solvent.

This thermally regenerated solvent process removes $\mathrm{CO}_{2}, \mathrm{H}_{2} \mathrm{~S}$ and other sulfur components. The amines are more selective to $\mathrm{CO}_{2}$ and therefore the hydrocarbon, $\mathrm{H}_{2}$ and $\mathrm{CO}$ losses are minimal in this process. MEA is the solvent of choice for flue gas operations given the low $\mathrm{CO}_{2}$ partial pressure because it has the fastest reaction rate. The disadvantages of MEA are: (1) it is has the largest heat of reaction which translates to the largest solvent regeneration heat duty; and, (2) it has the highest corrosivity and degradation rate. Tertiary amines have the lowest heats of reaction, corrosivity and degradation rates while the secondary amines are intermediate. The main disadvantage of tertiary amines is their slow reaction rates which lead to larger residence times and equipment costs.

Mitsubishi Heavy Industries has introduced a hindered amine solvent which they claim has a higher $\mathrm{CO}_{2}$ absorptivity, lower energy requirement, lower degradation rate and lower corrosion rate.

Reactive inorganic solvent based processes. Reactive inorganic solvent based systems (such as the UOP Benfield ${ }^{\mathrm{TM}}$ process) utilize an aqueous solution of potassium carbonate $\left(\mathrm{K}_{2} \mathrm{CO}_{3}\right)$, a solution activator, and corrosion inhibitors. The relative concentrations of these species, and hence the composition of the solvent, can be customized to the requirements of each installation. Potassium carbonate reacts with $\mathrm{CO}_{2}$ to form potassium bicarbonate, thus separating and removing the acid gas from the feed. The solution activator increases the $\mathrm{CO}_{2}$ absorption rates, thus decreasing column sizes and process heat requirements. The corrosion inhibitor allows for mostly carbon steel metallurgy. The combined $\mathrm{K}_{2} \mathrm{CO}_{3}$, activator, and corrosion inhibitor solution is typically used in oxygencontaining gas streams such as flue gas. The reactive inorganic solvent systems are not very efficient for low partial pressures of $\mathrm{CO}_{2}$ and have relatively high heat duties for regeneration.

Physical absorption processes using an ether solvent. Physical absorption-based processes include the Selexol ${ }^{\mathrm{TM}}$ process from UOP, which uses a polyethylene glycol ether manufactured by Dow Chemical, and the Rectisol ${ }^{\mathrm{TM}}$ process from Linde and Lurgi, which uses cold methanol. Compared with other absorbents, the Selexol ${ }^{\mathrm{TM}}$ solvent is 
more tolerant to contaminants and does not degrade as readily. It also has a much lower energy requirement for solvent regeneration since it involves breaking only a physical bond. The physical absorption process needs a high $\mathrm{CO}_{2}$ partial pressure and is therefore the process of choice for IGCC applications. It is not suitable for flue gas applications because the low $\mathrm{CO}_{2}$ partial pressure leads to high solvent inventories and high capital costs.

\section{Technology Enhancements}

A number of enhancements for amine-based $\mathrm{CO}_{2}$ removal are being investigated. Mixtures of solvents such as MEA and diethylamine (DEA) are being explored to reduce solvent energy requirements. . Sterically hindered amines require $20 \%$ less energy than the conventional MEA process. ${ }^{1}$ Aresta $^{2}$ has recently studied novel amines in order to increase the efficiency of amine solvents.

Process improvements are also under development in order to reduce the capital and energy costs of the amine solvent system. A membrane contactor to improve the gasliquid transfer rate and reduce the size of the absorber and stripper is being developed, as is a hybrid membrane-absorption process. An important aspect in $\mathrm{CO}_{2}$ capture process development is integration with the power station cycle. For example, a sorbent that can withstand relatively high temperatures will reduce the need to cool the flue gas. ${ }^{3}$

A further improvement of the amine system is to disperse the amine on a support in order to reduce the volatility of the amine solvent and thereby minimize loss. A number of researchers have adsorbed amines on sorbents such as carbon molecular sieves (UOP Patent US 4,810,266) and dispersed amines on polymeric materials like Amberlyst resins (US 5,876,488) ${ }^{4}$. These materials have limited thermal stability. Researchers have also immobilized amines on sorbents. For example, McMahan ${ }^{5}$ has immobilized amines on oxidized fly ash and activated carbon. The material adsorbs $\mathrm{CO}_{2}$ by a combination of both physical and chemical adsorption processes. The sorbents are limited to a maximum temperature of $80^{\circ} \mathrm{C}$. Aresta ${ }^{6}$ has recently reported the use of xerogel-immoblized amines for separating $\mathrm{CO}_{2}$ from gas mixtures.

\section{Current technology positioning}

The overall $\mathrm{CO}_{2}$ capture market was divided into several different segments, including natural gas, synthesis gas, chemicals, cement production, and power generation. For this study, we will examine and discuss only generalized results from the power generation segment, with a particular emphasis on coal-fired power plant $\mathrm{CO}_{2}$ capture.

The first part of the analysis was to collect customer requirements. For the power generation segment, these are listed in the first column of Table 1 below. The data in Table 1 were generated from lengthy internal discussions as well as Voice of the Customer (VOC) interviews with a few individuals associated with the post-combustion market segment. Note the "units" describing each requirement are provided in the second column. Of course not all customer requirements are created nor valued equally, so the next step was to rank the relative importance of each requirement to the customers. This is an extremely complicated task, and as such, results can vary somewhat from 
customer to customer. Nevertheless, we have endeavored to group the requirements into four groups of relative importance to the most typical, average, and generic power generator. These groupings appear in the third column of Table 1.

Among the most important customer requirements are high on-stream time, low footprint, and high product quality. It is expected that each and every technology described herein will have to adhere to these top three requirements in order to succeed. Also, it is very important to essentially all potential customers that the technology to be installed has been demonstrated at commercial scale. This means it will be more difficult for the next generation technologies to break into the marketplace than some so-called current offerings. Ease of operation and operation cost come next in the rankings, while capital investment and maintenance costs come somewhat lower on the list. The 'gee-whiz' factor of the technology and other local concerns rank low to customers in general.

\begin{tabular}{|l|l|c|}
\hline Customer requirements & units & \multicolumn{1}{|c|}{$\begin{array}{c}\text { relative } \\
\text { importance }\end{array}$} \\
\hline High on stream time & Days per year & 1 \\
\hline low foot print & $\mathrm{ft} 2$ & 1 \\
\hline Product quality & $\%$ of acid gas & 1 \\
\hline Demonstrated at commercial scale & rank & 1 \\
\hline Low Operation cost & $\$ /$ SCF & 2 \\
\hline Simple to operate & Number of skilled operators & 2 \\
\hline Hydrocarbon recovery & $\%$ of HC in product/feed & 2 \\
\hline Low Capital cost & $\$ /$ SCF & 3 \\
\hline Low environmental footprint & PPM/SCF & 3 \\
\hline Maintenance & $\$ / y e a r$ & 3 \\
\hline Quality of CO 2 & $\%$ impurities & 4 \\
\hline Local Content & rank & 4 \\
\hline Differentiated solution & rank & 4 \\
\hline
\end{tabular}

Table 1. Power generation segment customer requirements for $\mathrm{CO}_{2}$ capture.

The next step in the analysis was to compare and contrast each technology across each of the customer requirements listed in Table 1. Among the technologies evaluated were chilled ammonia, physical solvents, facilitated membranes, low temperature adsorbents (such as MOFs), high temperature adsorbents, ionic liquids, inorganic solids (e.g., solid carbonate), hot potassium carbonate solution, oxyfuel, algae, $\mathrm{CO}_{2}$ re-use w/o separation, chemical looping, generic amines, and specialty amines. For example, it is expected that customers would be much more willing to accept amine technology than a next 
generation technology such as ionic liquids because amines for $\mathrm{CO}_{2}$ capture has been demonstrated at commercial scale. On the other hand, next generation technologies like MOFs might be easier to retrofit into an existing power plant than other technologies. A summary of the overall technology assessment is provided in Figure 2. In order to generate Figure 2, each customer requirement in Table 1 was assessed for each technology type in a matrix fashion, and a single score was obtained for each technology.

As can be seen in Figure 2, the MOFs rank the highest of the next generation $\mathrm{CO}_{2}$ capture technologies and third-highest overall. Eventually, MOFs may be preferred over amines owing to being less toxic and non-corrosive and over carbonates owing to much more favorable mass transfer properties than either the solid or the solution form of these absorbers. Note that MOFs are physical adsorbers rather than chemical absorbers like the amines and carbonates. This means that the energy penalty to regenerate the MOFs should be less than for the amines or carbonates. Of course large beds of MOFs likely will be required because of the massive flow of flue gas at low pressure.

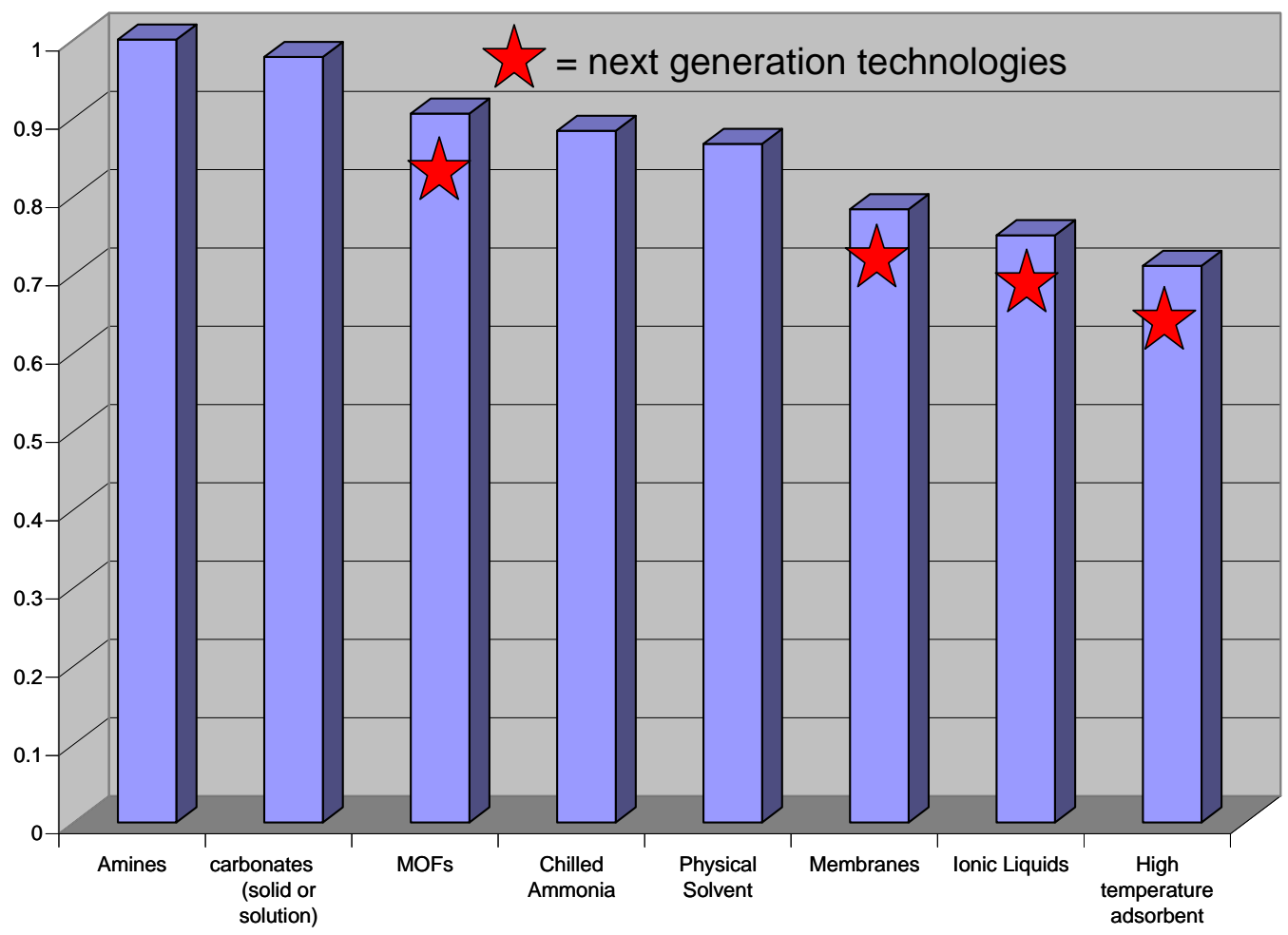

Figure 2. A relative ranking of current and next generation $\mathrm{CO}_{2}$ capture technologies. 


\section{$\underline{\text { Entry Barriers }}$}

Some barriers to entry into a new adsorbent application are listed here. It should be noted that this is not meant to be a comprehensive listing, but rather a set of potential barriers that could arise moving forward with MOFs for $\mathrm{CO}_{2}$ capture. The most important topics to consider here are the creation of internal capabilities, the validation of the application, and the commercial terms for the customer.

\section{Creation of internal capabilities}

Supply chain. Sometimes new adsorbent materials manufacture must be out-sourced. An internal or external supply chain must be established and proven. Often that supply chain may require certifications based on the industry standards for the application. As an example, ISO certification of the entire supply chain might be required for adsorbents going into the automotive industry. Since a new application often cannot justify the capital for dedicated manufacturing equipment, the new adsorbent must be made in flexible equipment used to make other products.

Design capabilities. We must know the new adsorbent's performance at each customer's process conditions. This means we must create models and design tools to predict product performance under whatever unique process conditions are presented by a given customer.

Industry standards. The application must be designed to the requirements of the industry. A unit which is not designed according to ASME standards would never be put into a chemical plant, no matter how inherently safe it might be. A flue gas application must be designed to the standards of the power industry. Team consultant EPRI has been helpful in providing some of this information.

\section{Validation of the application}

Commercial performance. It is great to have something work very well in the lab, but unless it operates with real feeds under actual conditions, it will not be viable. We need to know the pre-treatment requirements, and the expected performance over the life of the adsorbent.

Depending on the industry, the customer may require a demonstration unit. As mentioned above, this is very likely in the power generation industry. This could be a slip stream, but it may be a full commercial operation. The military has very well established requirements for commercial demonstration before something can be used in a particular application. Offshore platforms have requirements for commercial use before a technology can be included in a bid. Just like every refinery is different and has different requirements for technology demonstration, individual power plants have their own technology demonstration requirements to be met before they will consider incorporating a new technology. UOP will need to become aware of these particular requirements for each plant as we move forward with this technology. 
Process Integration. It is critical that the new adsorbent process fit in the current or proposed process flow scheme. Proving that the adsorption process can be put into a larger process with only well-known effects on the upstream and downstream parts of the process is critical to acceptance.

\section{Commercial Terms}

Customer value creation. We must understand how much value we create for the customer especially compared to alternative technologies. There are many discoveries which do extraordinary things in the lab but do not make economic sense for the customer to adopt. We can only optimize the value we create by understanding the customer's value proposition. There are often trade-offs in how we design a system such as opex versus capex which can only be made to work if we understand how the customer sees these trade-offs.

Risk mitigation. All new applications involve risk. While the exact risks are unknown, they often are related to the known risks of similar applications. Unless these risks are identified and mitigation strategies employed for both UOP and the customer, an application cannot go forward.

Sustainability. Both the supplier and the customer are making investments in the new technology. Unless there is some assurance of the sustainability of the application, it will not go forward. The customer does not want to buy a technology if there is a cheaper alternative just around the corner which their competitor will purchase. Meanwhile, we do not want to invest in creating the infrastructure for a "one off" application which will never be sold again, or quickly displaced by a cheaper alternative.

\section{Commercialization Pathway}

A description of the commercialization pathway, potential customers, market potential, and a projected market share based on fundamental market analysis. Consideration of economic, complexity, and industry risk aversion shall be compared.

At over 25\%, the power generation segment is responsible for emitting more carbon dioxide than any other segment. ${ }^{7}$ It is also expected that this segment will be the leader in utilization of $\mathrm{CO}_{2}$ capture technology. Here we will attempt to provide a very crude estimate of how MOF based systems might be used in $\mathrm{CO} 2$ capture from power generators.

When performing such an analysis, the first question is how MOFs will compete with other technologies. For example, as shown in Figure 3 (overall $\mathrm{CO}_{2}$ capture potential not just post-combustion), membranes are already an established technology for $\mathrm{CO}_{2}$ capture at natural gas fields. UOP Selexol ${ }^{\mathrm{TM}}$, a physical solvent, is the first generation $\mathrm{CO}_{2}$ capture technology for pre-combustion $\mathrm{CO}_{2}$ capture at power and chemical plants. One way to categorize MOFs is as next generation or "novel," meaning that MOFs do not exist in any application. To put this in context, we have assumed that the technology mix in $\mathrm{CO}_{2}$ capture will change over time. Further, we have assumed that chemical solvents 
will be replaced by Novel technologies such as MOFs, algae, new types of membranes (counted as Novel in Figures 2 and 3), enzymes, etc., and take a share of the rapidly increasing demand. The relative mix over time is shown in Figure 3.

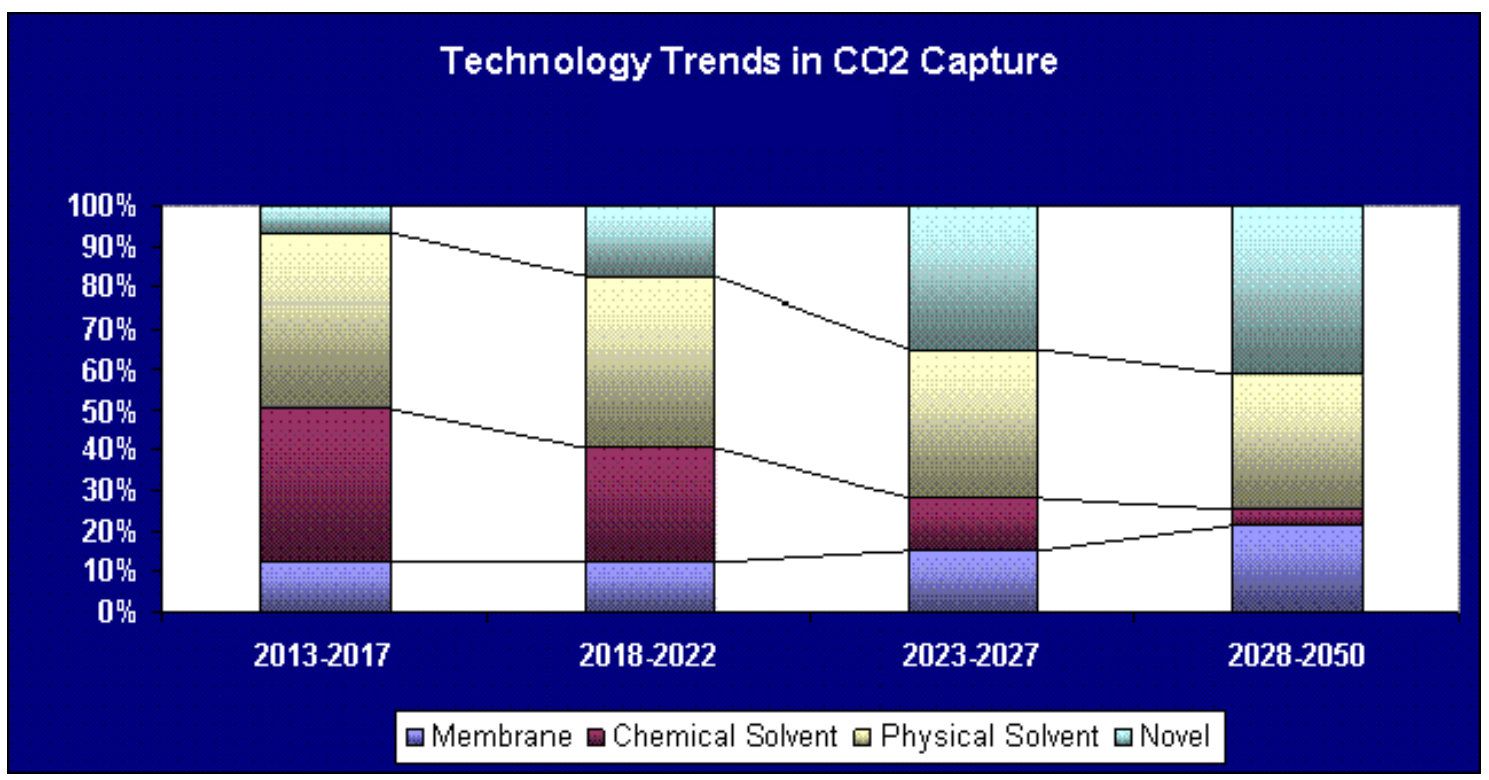

Figure 3. Potential technology trends in $\mathrm{CO}_{2}$ capture over the next 40 years.

As can be seen in Figure 3, physical (e.g., UOP Selexol ${ }^{\mathrm{TM}}$ ) and especially chemical solvents (e.g., amines) will lose market share to membranes and especially Novel technologies over the next 20 to 40 years. This will occur owing to two factors: (1) the number of post-combustion $\mathrm{CO}_{2}$ capture technology installations will begin to outnumber pre-combustion installations that utilize physical solvents, hence slowly shrinking the Physical Solvent piece of the overall $\mathrm{CO}_{2}$ capture pie, and (2) direct replacement of amines by Novel technologies in post combustion.

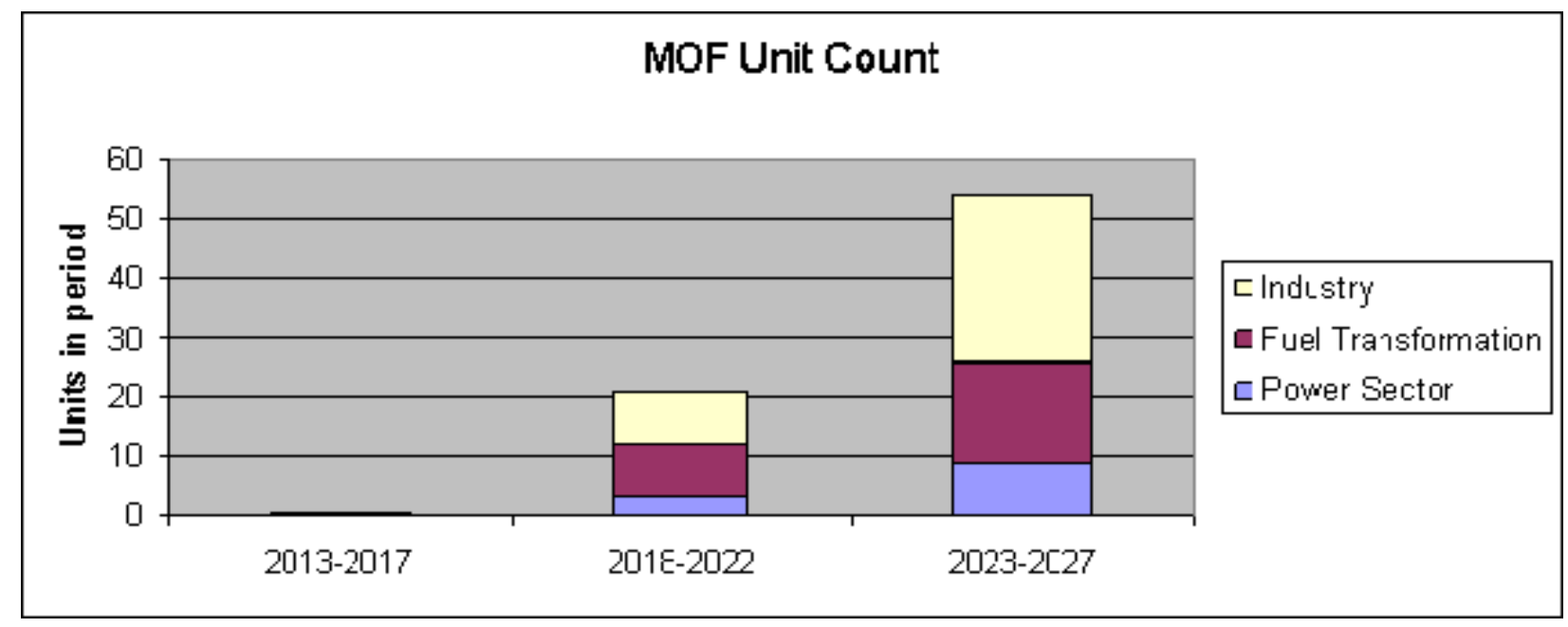

Figure 4. The number of MOF-based $\mathrm{CO}_{2}$ capture units in operation during the next 20 years. 
Our analysis presumes that MOFs will be among the Novel technologies that will capture increased market share. In fact, for this analysis we have assumed that MOFs can achieve $20 \%$ of the demand that will be met by the Novel technologies. We show the estimated unit count (Figure 4) and total separation capacity (Figure 5) spread over three market segments: Power, Fuel Transformation (such as tar sands) and Industry.

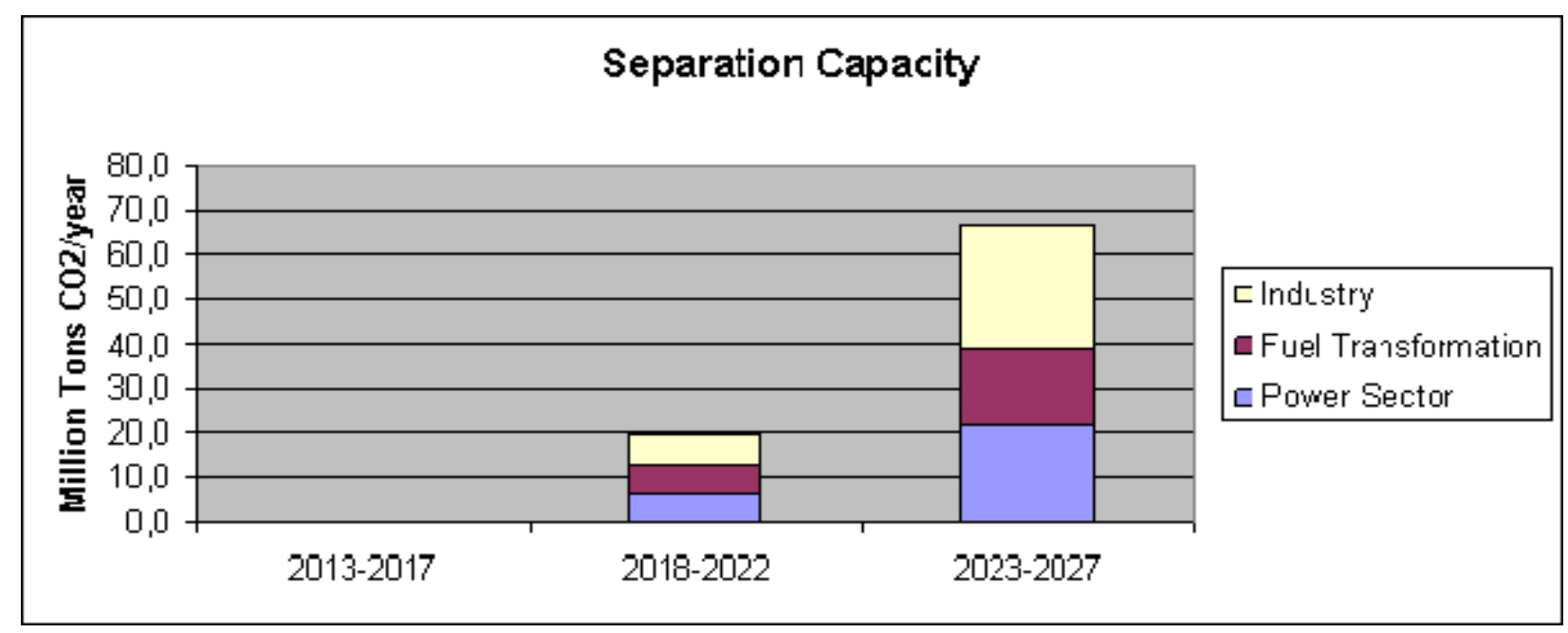

Figure 5. The total $\mathrm{CO}_{2}$ separation capacity over three market segments for the next 20 years.

\section{Obviously this is one scenario. It is not meant to be an estimate of business, but rather it gives some idea of where we would expect to develop the business over what timeframe and what size it might become.}

With a potential market for MOFs for $\mathrm{CO}_{2}$ capture identified, it is possible to calculate how much MOF material will be required to fill the commercial $\mathrm{CO}_{2}$ capture units described above. Utilizing real team-generated data (several $\mathrm{CO}_{2}$ isotherms at various temperatures) on one of the team's best performing MOFs, it was estimated that up to one million pounds of MOF material will be required per $550 \mathrm{MW}$ power generation plant. The analysis utilizes a very conservative $10.5 \mathrm{wt} \% \mathrm{CO}_{2}$ working capacity, at 8 minutes cycle time, and $36{ }^{\circ} \mathrm{C}$ as inputs.

Any new capacity needs are based on the total UOP business at the time, meaning that estimates depend upon what is happening throughout the UOP products business. These will not be provided at this time. In general, the adsorbent lifetime will be on the order of a few years. If in reality the lifetime is significantly shorter, then additional manufacturing capacity likely would have to be added. 


\section{Technology Transfer Alternatives}

As an established developer of novel materials, UOP has significant experience in bringing this type of technology to market. In the past, UOP has employed a variety of models to generate value from its intellectual property.

For the novel materials, UOP may choose to synthesize the materials in-house or may opt to have them toll-manufactured. Factors impacting that decision are: the type of synthesis; how easily it can be accommodated in UOP's existing production lines; the availability of the production line; the extent of trade secret protection in the synthesis; the synthesis cost versus price to the end-user. In either case, UOP would then sell this MOF material as a specialty product. UOP would typically offer a performance guarantee, included in the price of the material to the customer. In the current case, MOF synthesis is close to UOP's conventional molecular sieve synthesis techniques, so UOP may opt to make the material in-house. Due to the large volumes of materials required in these applications, UOP would have to consider significant expansion of its production capacity (see previous section).

An adsorbent reload model would further be considered as part of the value proposition. Adsorbents typically exhibit a certain limited life (not yet established in the MOF test program) in which they operate according to specification. Once they no longer perform adequately, a new batch of adsorbent, of the same or a new generation, would be offered for sale.

The process design, currently thought to be variation on our pressure swing adsorption (PSA) technology, can be brought to market as a 'Schedule A', i.e., an engineering design that can then be detailed and built by an Engineering, Procurement, and Construction (EPC) company. In that case, the technology transfer to the customer would take place in the form of the design and a licensing event. If there is specific key mechanical equipment (KME) involved, UOP may further choose to build that equipment in house and specify that equipment separately.

Alternatively, UOP might consider modularization as in, e.g., skid-mounting, and would sell the whole process as a piece of equipment. As with the sale of materials, UOP will likely offer a performance guarantee as part of the licensing event, with the sale of the $\mathrm{KME}$ or the sale of modular equipment. In the current case, the large volumes of gas that require treating do not suggest that modularization is the preferred option. A Schedule A approach appears to be more likely.

Technology transfer options that are not typically considered by UOP could potentially be considered in this case too. A Build-Own-Operate (BOO) model in which UOP is contracted to ensure a certain $\mathrm{CO}_{2}$ capture specification may be considered. Management of $\mathrm{CO}_{2}$ credits with appropriate agencies may offer a further extension of that model.

For the current value proposition evaluation, a conventional one-time sale of adsorbent, made in-house, would be coupled to a Schedule A/licensing event. 


\section{Commercialization Timeline}

- A commercialization timeline with associated funding requirements, industry partnership requirements, and cost reduction projections at each stage of development.

Adsorbent timeline. At UOP, typical new materials synthesis to commercial scale adheres to the following development scale-up steps:

1. Synthesis at $100-200 \mathrm{~g}$ scale

2. Synthesis at $1-2 \mathrm{~kg}$ scale

3. Synthesis at $20-30 \mathrm{~kg}$ scale

4. Commercial Trial at $4,000-7,000 \mathrm{~kg}$ scale

Exactly how and where a new material is manufactured depends on how the prototype is developed and what critical aspects of the recipe are needed to meet the performance targets of the product. For example, if the MOF can be synthesized at UOP and formed into a suitable size and shape adsorbent by standard synthesis and extrusion processing, the entire preparation could be done internally. But if some aspect of the preparation strays from standard operation, then capital for another manufacturing line or utilizing an outside specialty manufacturer (tolling) may be necessary (see earlier Commercialization discussion).

The total cost of development, as described above and excluding any research carried out before the development effort begins, is estimated to be about $\$ 5$ million per year and could take around five years to complete.

Process timeline. Step 2 above would provide enough material to start up a dual-bed vPSA pilot plant operation. However, full-time pilot plant operation would likely not commence until Step 3 was completed on at least one material. After sufficient adsorbent material quantities were obtained, process variable and other critical process assessments would be carried out on realistic feeds on an on-going basis. Presuming a year or two of continuous operation (at about $\$ 5 \mathrm{MM} /$ year), the business would then need to decide, based upon the data from the pilot plant, whether UOP is ready to propose a 2-3 MW slipstream test or jump directly to a larger scale demo unit $(\sim 50 \mathrm{MW})$. In either case, we would have to be at the Step 4 scale of Product development in order to outfit the slipstream unit and especially the demo unit with sufficient material to do $\mathrm{CO}_{2}$ capture. It is expected that a slipstream vPSA operation would take six months to a year for UOP to set up, and then operate for up to a year to generate data required to go to the next level. All the while, extensive process modeling would be required. The cost would be around $\$ 5-10 \mathrm{MM} /$ year, depending upon several variables. Demo scale operation is highly variable, both in terms of time and dollar requirements. It is impossible to commit to time and cost figures for a demo unit at this time. However, since UOP has extensive experience in the establishment of demo units, the expectation is that the construction and operation would proceed in a straightforward manner. 


\section{$\underline{\text { Summary }}$}

This report summarizes UOP's preliminary assessment of a MOF-based vPSA process for $\mathrm{CO}_{2}$ capture from coal-fired power plant flue gas. We believe our technology is equal to, or perhaps more, technically feasible than the other current and emerging technologies for flue gas $\mathrm{CO}_{2}$ capture. For example, MOFs offer high capacity, high mass transfer rates, and ease of regeneration at typical flue gas temperature and pressure. Early indications suggest that $\mathrm{MOF} \mathrm{CO}_{2}$ selectivities over other flue gas components are reasonable, and that MOF contaminant stability may be manageable. Owing to UOP's over 90 years of commercial success, our analysis of key entry barriers and technology transfer options leaves us well positioned with several available options to shepherd the proposed technology to market. The likely commercialization pathway involves many steps and presumes it will not likely be started without a government mandate to reduce $\mathrm{CO}_{2}$ emissions. Nevertheless, we describe how we believe the MOFs could establish and grow a certain market share and further, what it will take from a manufacturing point of view to prepare the MOF adsorbent to fill the needs of the power generators. We also report herein that once we have decided to move forward with MOF commercialization, about \$5 MM per year will be spent on "development" costs over several years to bring the MOF adsorbent and vPSA carbon capture technology to market.

\section{References (APPENDIX 4)}

\footnotetext{
1 "Development of energy savings technology for flue gas carbon dioxide recovery in power plant by chemical absorption method and steam system" Mimura T., Simayoshi H., Suda T., Iijima M. Mituoka S., J. Energy Converisons and Management 38, S57-S62.

2 "Carbon dioxide capture by amines: increasing the efficiency by amine structure modification"

Dibenedetto A., Aresta M., Abstracts of Papers, 223rd ACS National Meeting, Orlando, FL, April 7-11, 2003.

3 "A technical, economic, and environmental assessment of amine-based $\mathrm{CO}_{2}$ capture technology for power plant greenhouse gas control” Rao A.B., Rubin, E.S. Environ. Sci. Technol. 6ㅜ, 4467-4475, (2002).

4 “'Regenerable solid amine sorbent,' Birbara P et al., US Patent 5,876,488.

5 "Amine enriched solid sorbents for carbon dioxide capture” McMahan L.G., Soong Y., Champagne K.J., US Patent 6,547,854.

6 "Xerogel-immoblized amines as a new medium for $\mathrm{CO}_{2}$ separation from gas mixtures" Aresta M., Dibenedetto A., Girardi R., Abstracts of Papers, $225^{\text {th }}$ ACS National Meeting, New Orleans, March 23-27, 2003.

7 "Navigating the Numbers: Greenhouse Gas Data and International Climate Policy." Baumert, K.A., Herzog, T., Pershing, J. World Resources Institute: 2005.
} 